Refirence

A]1101 785409

NBS
Publi-
cations NATL INST OF STANDARDS \& TECH R.I.C.
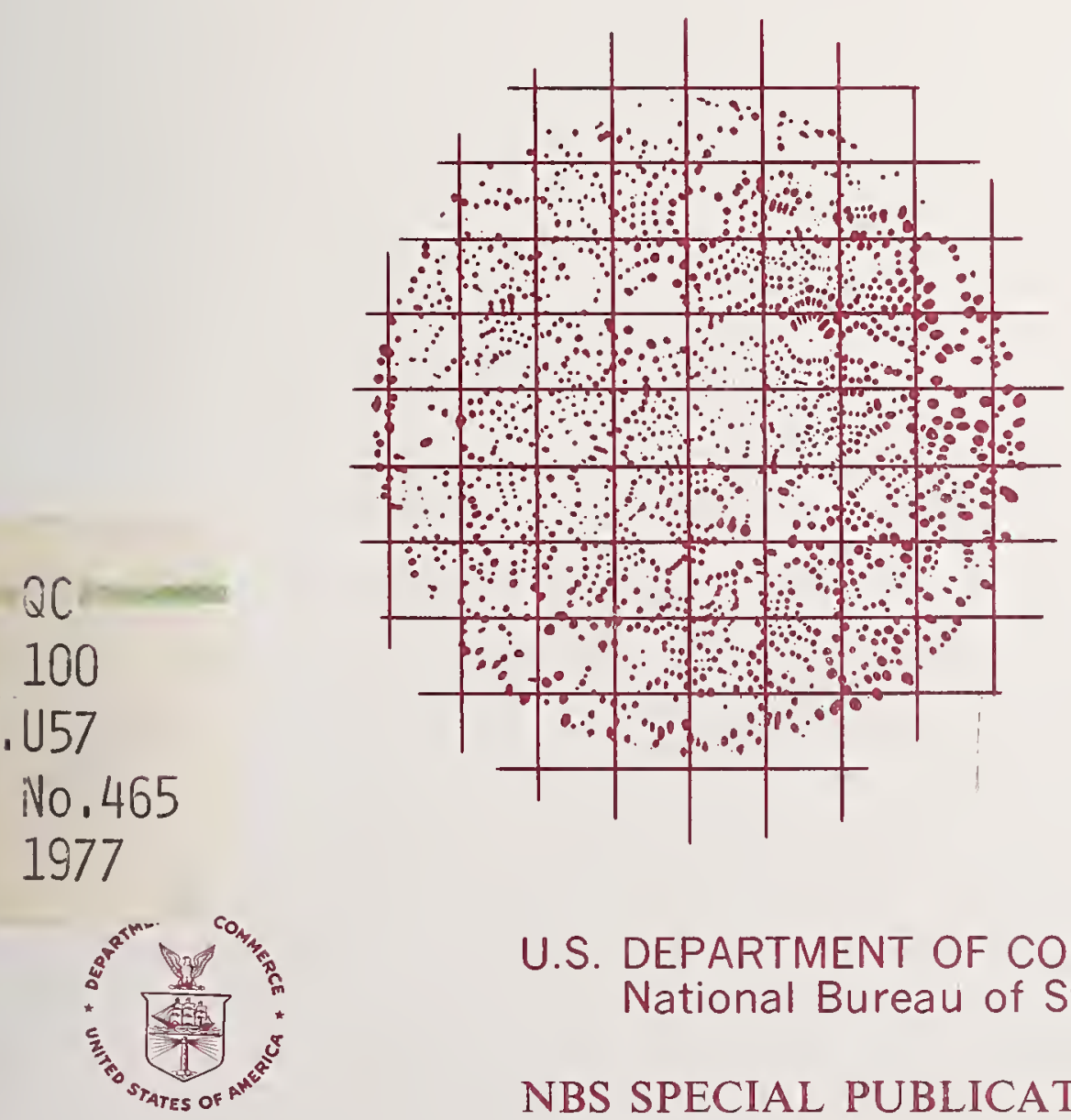

U.S. DEPARTMENT OF COMMERCE National Bureau of Standards 




\title{
Science and Technology in America
}

$772133-\operatorname{Rop}$
$Q(100$

\section{an assessment}

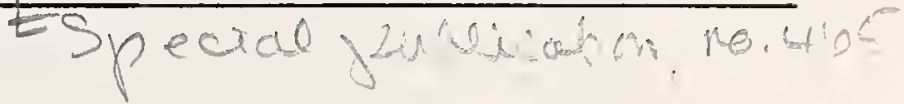

\author{
by Edward Teller \\ Norman Ramsey \\ William 0. Baker \\ Henry Eyring \\ Garrett Birkhoff \\ Alan Perlis \\ Robert C. Seamans, Jr. \\ Simon Ramo
}

Edited by

Robb M. Thomson

National Bureau of Standards

Washington, D.C. 20234

The Distinguished Lecture Series

published on the occasion of the

75th Anniversary of the National

Bureau of Standards

\section{YEARS \\ NBS \\ 1901-1976}

U.S. DEPARTMENT OF COMMERCE, Juanita M. Kreps, Secretary

Dr. Sidney Harman, Under Secretary

NATIONAL BUREAU OF STANDARDS, Ernest Ambler, Acting Director

Issued June 1977 


\section{Library of Congress Catalog Card Number 76-600073}

National Bureau of Standards Special Publication 465

Nat. Bur. Stand. (U.S.) Spec. Publ. 465, 166 pages (June 1977) CODEN: XNBSAV 


\section{FOREWORD}

This monograph, "Science and Technology in America-An Assessment," is the published version of a series of distinguished lectures delivered at the National Bureau of Standards during the months of March and April 1976. This series was conceived to commemorate two anniversaries - the birth of this Nation 200 years ago, and the founding of the National Bureau of Standards 75 years ago. With this monograph, we are happy to share the authoritative view which the series as a whole provides of the broad reach of science and technology in this country with a wider audience than was able to attend the lectures in person.

This Series was designed to assess the current state and future prospects of those broad physical sciences and technologies in which the Bureau of Standards is significantly involved. Science and technology have been increasingly a critical factor in the development of our world. More specifically, this Nation was born in an age of scientific exploration and experimentation. Some of the signers of the Constitution, who considered themselves men of science, included Franklin, Madison, Pinckney and Jefferson. These men looked to the early establishment of a national university and Federal societies of the arts and sciences.

Over the past 200 years, we as a Nation have participated increasingly in the development of the intellectual structure we call science-an achievement in its own right. By expanding our knowledge of nature in this way we are, in fact, expanding knowledge of ourselves. For in the end the primary resource for, and the primary beneficiary of, science and technology is man himself.

Resistance to technological innovation is certainly not a modern development. The early restrictive and nuisance laws on horseless carriages vis-a-vis the horse-drawn variety is but one case in point. However, the pace of modern technological innovation and the ultimate limits in global carrying capacity, which come ever closer upon us with each increment in production and population, have caused us to look at science and technology with new eyes. Some propose returning to a simpler life with a moratorium on science and technology. Others point to the need for continued scientific and technological growth, but call for greater understanding and assessment of the overall effects and externalized costs of major new technological deployments. Clearly we are in a period which demands much greater statesmanship from the scientific and technological community, and we at the National Bureau of Standards believe we have contributed much, and have much more to offer in the future along this line. 
However, as practicing scientists, we also believe that the scientific quest is as great a challenge as it ever was, and that the deep personal satisfactions which come to the creative scientist still speak as strongly as ever.

In this light, then, we at the National Bureau of Standards are proud to present this monograph which is both an assessment and a celebration of science and technology in America.

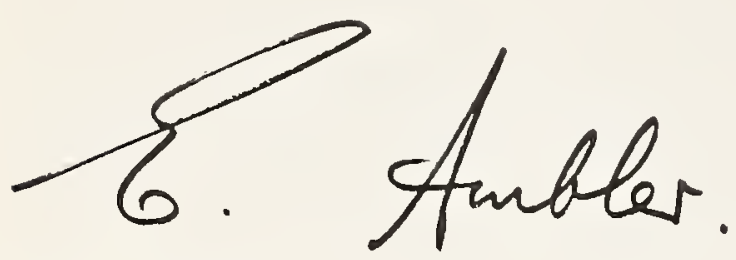

Ernest Ambler

Acting Director, National Bureau of Standards 


\section{CONTENTS}

Science and Technology in America:

A Chronicle and Some Predictions

Dr. Edward Teller

Lawrence Livermore Laboratory

Quantum Science and Its Impact on Metrology

Professor Norman Ramsey

Harvard University

Materials

Dr. William O. Baker

Bell Telephone Laboratories

Chemistry - A Changing Frontier

Professor Henry Eyring

University of Utah

Applied Mathematics and Its Future

Professor Garrett Birkhoff

Harvard University

Computers in Science and Technology

Professor Alan Perlis

Yale University

The Technology of Energy

Dr. Robert C. Seamans, Jr.

Energy Research and Development Administration

The Future Role of Engineering

Dr. Simon Ramo

TRW, Inc. 


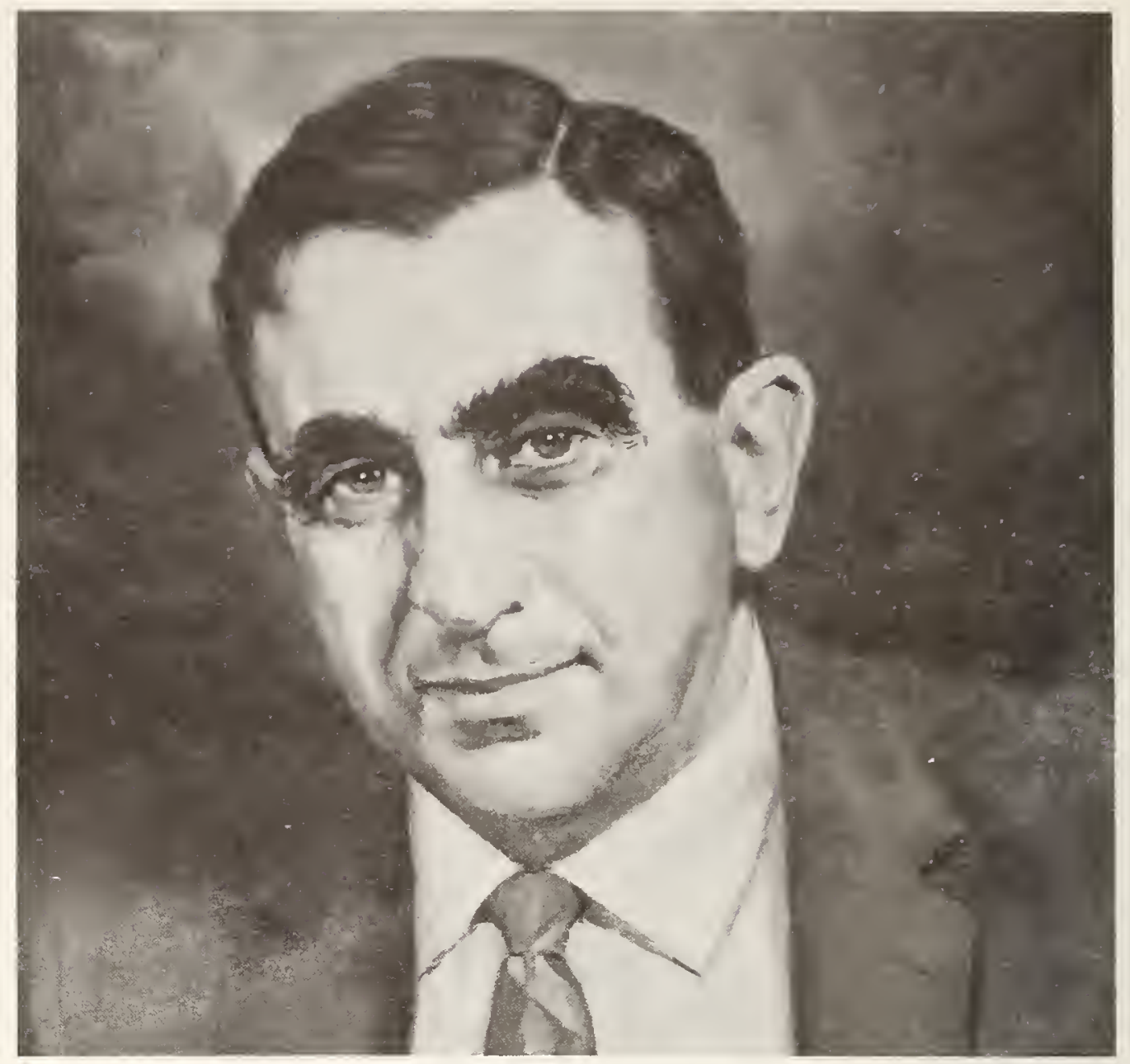

Dr. Edward Teller is University Professor Emeritus of the University of California, Associate Director at Large Emeritus of the Lawrence Livermore Laboratory, and Senior Research Fellow of the Hoover Institute on War, Revolution, and Peace of Stanford University. He has made pioneering contributions in a wide range of science and technology. His wellknown work in the development of thermonuclear weapons was preceded by earlier fundamental work on thermonuclear reactions. He has also made major fundamental contributions to the quantum theory of polyatomic molecules, and still maintains a continuing interest in molecular and nuclear physics. In addition, Dr. Teller helped develop national programs to explore methods of processing thermonuclear energy by means of both magnetic confinement and laser techniques. $\mathrm{He}$ is a member of the $\mathrm{Na}$ tional Academy of Sciences, and the Commission on Critical Choices for Americans, the President's Foreign Intelligence Advisory Board, and the Board of Directors, University of Tel Aviv. He has received the Joseph Priestly Memorial Award, the Albert Einstein Award, the Enrico Fermi Award, and the Harvey Prize of the Technion of Haifa. 


\section{SCIENCE AND TECHNOLOGY IN AMERICA: A CHRONICLE AND SOME PREDICTIONS}

Seventy-five years ago when the Bureau of Standards was established, a very great change occurred, perhaps by coincidence, in the field which I will address. Up to that date there was almost no connection between science and technology. Even though technology, of course, used the results of science, a real collaboration existed only as an exception. Today we can hardly speak about either science or technology without mentioning the other.

I would like briefly to go back into the dark ages in America, when science and technology still went their separate paths. I want to emphasize technology throughout my lecture, even though I came to the United States as a pure scientist and was converted slowly and painfully to technology. Technology in America was something very remarkable because it was so different even then from technology anywhere else. I would like to highlight this by recording a few facts and relating a few stories.

My first fact is: We are accused today of an indecently high per capita consumption of energy in relation to the rest of the world. In the year 1800, when America was an agricultural society, our energy consumption was even more out of line than it is now. In 1800 the per capita energy consumption in the United States was almost as high (I think about $80 \%$ as high) as it is now in Western Europe. The energy was, of course, mostly derived from wood, which was there for the burning; the more you burned, the better. The other source was hay for the horses and "horsepower." This latter provided one-third of the energy. But energy even at that time was consumed in the United States much more copiously than it is today almost anywhere in the world.

Apart from being so rich in energy we have a very peculiar problem in this country: size, and the difficulties which come with physical size. One very interesting story of how size has affected America is connected with the Governor of New York and the attempt at the beginning of the 19th century to open up the Far West (meaning Buffalo). As Governors of New York usually do, DeWitt Clinton established a commission. On that commission there served a man by the name of Colonel John Stevens, who said, "I have heard of a man in England; I think his name is James Watt. He has built a steam engine. In this country, we have had rails in mines for a long time. Could we not put one of James Watt's engines on such rails in order to get to Buffalo?" Nobody believed him. Quite a few years later, and three years before Stevenson's "Rocket," Colonel John 
Stevens demonstrated the first locomotive on his own farm. But the New York commission said no.

The commission decided instead on the Erie Canal, and the proposal went to Washington for approval. The President of the United States at that time said that to dig a canal, hundreds of miles long, through wilderness, was an idea which was premature at least by a century. The President of the United States who said that was, surprisingly enough, Thomas Jefferson!

Whereupon the Governor of New York went ahead with the canal project by means of that marvelous recent invention of Adam Smith, private enterprise. The bonds were subscribed, the Canal was dug. It was not the longest canal in the world - the Chinese had done better almost 2000 years earlier. The Canal was finished in the 1820's, quickly paid off all its debts, and made a profit. But 15 or 20 years later, it was put out of business by competition with the railroad which had been suggested by John Stevens in the first place.

These days, when I go to bed, desperate because an additional incredible blunder has been made in Washington, I like to remember that story.

In those days, America was not yet industrialized. Still, it had probably as many steam engines as all of Western Europe. Because of the riverboats, and then later the railroads, steam engines were needed to overcome the distance. Another step in the same direction was the early use of the telegraph in the United States. It was much more important here than in Europe where communications over the shorter distances were much easier.

Among many others, there is a third specific accomplishment of the United States that I want to mention. This third accomplishment is tied to the name of Eli Whitney, and I do not have in mind the cotton gin. Eli Whit ney pioneered the proposition that one could get money out of the Defense Department. He got a contract for making guns. Not only did he make that breakthrough, he also failed to deliver in time, overran his budget and still continued to get money out of Washington. When the early predecessor of the Pentagon became impatient with him, asking him to bring in some guns - he arrived in Washington with a sack. When he opened the sack, there were no guns. Asked, "Where are the guns?" he said, "These are ten guns; just put them together." In the sack were interchangeable parts. Apart from some very early attempts in Venice, this was a brand new approach which Eli Whitney made to work only in a halting and ineffective way. But it was a beginning. From there, of course, came the bicycles and the sewing machines and the typewriters, and in the end, even Henry Ford's automobiles.

Not only was genius at work in the case of Eli Whitney; of that there can be little question. But there was also something else at work - something connected with necessity, and a reasonable reaction to 
a challenge. The industrial revolution - real industrialization - came to the United States only in the second half of the 19th century, even a little later than mid-century. The remarkable thing is that labor in the United States was then twice as expensive as in England, and in England, in turn, people were better paid than in continental Europe. Yet America managed to compete. The higher wages were, of course, due to the fact that anybody could go out West and get land. A man in America in those days did not need to work for miserable wages. But in spite of those high wages, or because of those high wages, American industry was forced into the path of innovation.

It is more remarkable that in 1862 , in the middle of a disastrous war, Congress should have taken time to establish the land grant colleges for the specific purpose of encouraging the agricultural and mechanical arts. I claim that this single act of Congress did more than anything else in the history of this country to establish the muscle of present day America.

But let me now turn to the 20th century. You will find there example after example of real collaboration between science and technology. The two have become practically one. My favorite example is flying. Of course, the first planes were put together by tinkerers, extremely ingenious tinkerers, whose experiments were not unrelated to the scientific method. However, very soon, and at first not primarily in this country, the more elaborate theory of aerodynamics and a beginning of a decent theory of turbulence brought science and technology into the closest relationship. The same holds, with a little time delay, for electronics, which was particularly strongly based in this country from the very beginning. Even today the electronics industry is the only industry - and forgive me for slightly exaggerating - which will undertake a research job without a certainty that it will pay off in three years.

Another example which I would like to mention is nylon, which I discuss for a peculiar reason. Nylon was developed as a weapon in the battle of the sexes. That was the original intention, and therefore it is rightly called a peaceful enterprise. But when it succeeded, the girls got no stockings, because World War II was on, and all the nylon went into parachutes and bomber tires. I tell this very short story to indicate that there is no invention, no new development that is either wholly peaceful or wholly warlike. Anything that we learn, anything that can be developed, can be used in a variety of ways. The modern idea that technology is dangerous may be partly right, but if you want to maintain such a claim you must carefully qualify it.

I have not mentioned the Bureau of Standards, and I would like to do so now very briefly, and from my limited point of view. I came to Washington in 1935, and made many friends here. I came to a new world, which is never an easy experience, but it was made very much easier not only by the hospitality of Americans, not only because they are much more tolerant 
toward all kinds of peculiar people, but also because the scientific community was completely open. In that community, one never even dreamt of any distinctions except those between good science and something not so good. During several fruitful years in Washington, I came often to the Bureau of Standards (although, of course, not to its present building). I came once every week because Fred Mohler had a colloquium which was the Washington colloquium. People came from all the surrounding universities, the Naval Research Lab, and the Carnegie Institution. I tried to contribute, both by helping to invite speakers, and by being less than completely restrained in asking questions. I felt that I had a chance to participate, through the Bureau of Standards' colloquium, in the scientific work of the Washington community.

I was asked in the recent past the question, how were the administrative problems of the Bureau of Standards at that time. And I confess that I knew absolutely nothing about them. Neither I nor anyone with whom I talked in those days mentioned bureaucratic problems. Big science had not yet been born, and at that time I was a pure scientist, fully enjoying the immense intellectual stimulation that this gave to all of us.

Then, of course, came the Second World War. And again I would like to tell a short story because it is connected with the Bureau of Standards. One of my crazy friends, another Hungarian, Leo Szilard, wrote a letter about the possibility of a nuclear bomb, to be signed by Albert Einstein. The letter was addressed to President Roosevelt. It arrived on Roosevelt's desk two or three months later, and he called the Director of the Bureau of Standards, Dr. Briggs, about it. Dr. Briggs held a meeting to explore the question, to which I was invited. That was the beginning of scientific bureaucracy. I will speak about only one aspect of this meeting because I have been given credit or blame for one of its results in a manner which is not quite justified. One of my friends who was also invited was Enrico Fermi. Fermi was at Columbia University, and he said he would not come to the meeting. Since I was his friend, I was sent up to New York with instructions to "Bring Fermi." I talked with him, and Enrico just wouldn't come. He had talked about the possibility of atomic energy to the Navy previously, and they had thrown him out, so he had had enough of such nonsense. I tried to argue, but Fermi was a pretty stubborn individual. In the end he said, "Edward, look, I can tell you what I would say if I went. You tell it for me. I don't need to go." And that's how it happened.

After some preliminaries at the meeting, a controversy developed between Szilard, who believed in nuclear bombs, and a colonel from Aberdeen who didn't. It was then my turn, and I very properly said, "I am only here to represent Fermi, and we want to go ahead; we know how to go ahead. We must first build a reactor, and in order to slow down the neutrons, we need graphite. But we need pure graphite, and pure graphite is not available. Therefore, we need a budget for the first year of $\$ 6000$." 
Well, Szilard almost murdered me on the spot, because $\$ 6000$ is what we got for the first year. This was the start of Scientific Bureaucracy and of Big Science.

I will not continue the story of atomic energy because that is history, even if much of it is secret. Apart from atomic energy I certainly should mention the field of computers and the exploration of space. Computers are machines which can do anything that the human brain can do provided only that we understand in real mathematical terms what the human brain does. They represent a tremendous opportunity not only in a practical sense, but also because we can through them imitate what the human brain does and in the end understand our own brain a little better. About space exploration, I want to be quite brief. We made a splendid start and then we stopped. However, I am very sure that we did not stop for good.

At this point $I$ have to mention a very terrible thing. Starting with Hiroshima (and I have clear and detailed evidence throughout the years that it started with Hiroshima), technology in the United States fell into disrepute. Some of our young people say technology is irrelevant. They did not invent this absurd statement. The professors invented it. I was present when a group in 1947 or 1948 gave advice to the Ford Foundation not to bother with technology or anything like it - not even with science. Concentrate, they said, instead on the social applications. That recommendation came from scientists. The young people learned it, and they started to hold in contempt not only technology, but in the end also science itself. As a direct consequence, 1970 or 1971 was the first year when the United States imported more manufactured goods than it exported. People blame it on high wages. The wages, relatively speaking, were even higher at the very beginning of American technology. But high wages can be paid if there is innovation. And it is on this front of innovation where we are lacking. Today the university departments of engineering have a low standing in academic counsels. It is a fact that institutes of technology, instead of taking pride in themselves, very frequently begin to call themselves universities. All of us in the scientific and technical community bear a great responsibility to be ever mindful of that essential relationship between technical innovation and ultimate economic well-being. I believe that one of the centers that has remained strong and vigorous throughout the years in the united field of science and technology is this Bureau of Standards.

I turn now to some predictions. What is next in science and technology? I am told that one shouldn't make predictions, particularly not about the future, but I prefer to live dangerously.

One of the predictions I make is that we must address the energy crisis in a substantive technological way. But we do not. I shall mention some details showing why we do not. The first point has to do with the time scale of research pay-off. Industry is doing a lot of good research that will pay off 
in the next three years. Government agencies usually concentrate on projects that will bear fruit in 15 to 20 years. Between the two lies a no man's land. The problems of energy cannot be solved in three years, but they can be solved in 10. Unfortunately, nobody is addressing the relevant technical issues in this 10-year time period. I am much concerned about this subject, because this is one of the really terribly important fields on which our strength and maybe even our national existence may ultimately depend.

I shall make a few specific remarks about the technology of energy, beginning with a little remark about fossil fuels. The most popular idea (and in a way it's rightly popular) is to gasify, liquefy and purify our abundant coal. ERDA submitted a marvelous program-\$6 billion for pilot plants which are copies of what was done in 1940 . Fortunately it was defeated in the House of Representatives. There are two much better possibilities. First, is the use of modern technology for operating gasification processes at very high temperatures. New materials are either now available or could be made available which can withstand these high temperatures. The second possibility is underground coal gasification and in situ conversion of oil shale into oil. These vital possibilities receive little attention.

Another point, even more important, is conservation. We have been spendthrifts for 200 years. I have a theorem for which I should get the Nobel prize. The theorem is that of all matter known to man the one with the highest inertia is the human brain; with one exception only, the collective human brains of the whole nation. If we have been spendthrifts for 200 years - then to break the habit is not easy. In industry as a whole, we don't know by what specific tricks we can save energy. Incidentally, the Bureau of Standards has done a small but marvelous job on better insulation for buildings, particularly of mobile homes.

Solar energy. We speak about the extensive use of solar electricity which is currently 10 times as expensive as electricity from other sources, and which will require large scale land use (which the environmentalists will oppose strenuously the moment we begin to introduce it). But we know today how to use solar energy to produce domestic hot water, and we know how to use solar energy to raise steam for process heat. In these fields we have barely started.

And last, but not least, I speak of nuclear energy. Our main effort goes into the fast breeder reactor, and specifically into building a fast breeder on the Clinch River. That breeder is obsolete even before the ground is broken. It is supposed to cost $\$ 2$ billion and eventually it will cost $\$ 5$ billion in the best tradition of Eli Whitney. But I do not think it has much else in common with Eli Whitney. There are plenty of other possibilities in nuclear reactors. There is the matter of the thorium cycle which is much easier to work with. In that case, we just have to produce heavy water. ERDA may begin to move in that direction. I would be happy about that. 
But the main problem is that propaganda for a nuclear shutdown is gathering force. Of course, we have an absolutely perfect health and safety record with industrial nuclear reactors. The trouble is in the minds of the critics. The trouble is in the minds of 2000 scientists (or people who call themselves so, but most of whom have never heard any details of nuclear reactors) who protest. These protests have come to a head on the 8th of June, 1976 in a California vote, and the issue will also come up in other states. Washington cannot be blamed for that, but it can be blamed for not doing something to help. We have excellent methods to dispose of wastes. However, a decision must be made. I am reminded of Buridan's ass who starved to death between two equidistant and equally desirable stacks of hay. We have in the nuclear waste field at least four solutions, all excellent, but we also have four bureaucracies to make the decision: NRC, ERDA, EPA and CEQ. These agencies are coupled together, and they must all go in unison to one solution.

Unlike my old days in Washington I seem to be drifting into administrative problems. I do not wish to finish on that note.

There are at least two tremendous tasks ahead of us which are scientific and technological in the best traditions of those words. One task is to understand the atmosphere, to predict the weather, and in the end to modify it. I believe it will be possible. It should be done in the United States. Considering our present antiscientific orientation, it may be done first in Russia. However, I must warn you that if we succeed in modifying the weather, something very remarkable will have happened. We will have lost our one safe topic of conversation.

The second big task is to make use of the oceans for oil, for manganese nodules, and more than anything else, for the production of food.

The historic importance of technology today is that it is spreading across the face of the world, and this spread is inexorable. Some people will always predict the end of the human race. This is nonsense. However, what is certain is that in a few decades the whole world will in some sense be technological. And this change, coming as suddenly as it will, in many cases to primitive peoples, may bring in its wake a number of cat astrophes. There is no softer word for it.

The first wave of this worldwide spread of the industrial revolution was in medicine. It was a very praiseworthy and most successful attempt to save the lives of infants. As a result we now have a population explosion three times as rapid (in exponential growth) as was the European population explosion of the 19th century. In the year 2000 we will have a world population very close to 7 billion people rather than the 4 billion we have now. I do not advocate the preaching of birth control. People will come to that on their own account. But no matter what we do about birth control, the people on earth in the year 2000 cannot be fed unless the old traditions of the land grant colleges are exploited worldwide, and unless the oceans, 
which today contribute only some 7 percent of the world's food supply, are thoroughly used in every possible way.

The big problem in the world today is energy. Our big problem for the next few decades will unavoidably be food. And that is why the study and exploitation of the ocean is something that deserves particularly great emphasis. None of these important things, important for a smooth transition into a decent future for all humanity, can happen without science and technology. It is thus essential for us to win the battle for science and technology if America is to have a third century. 


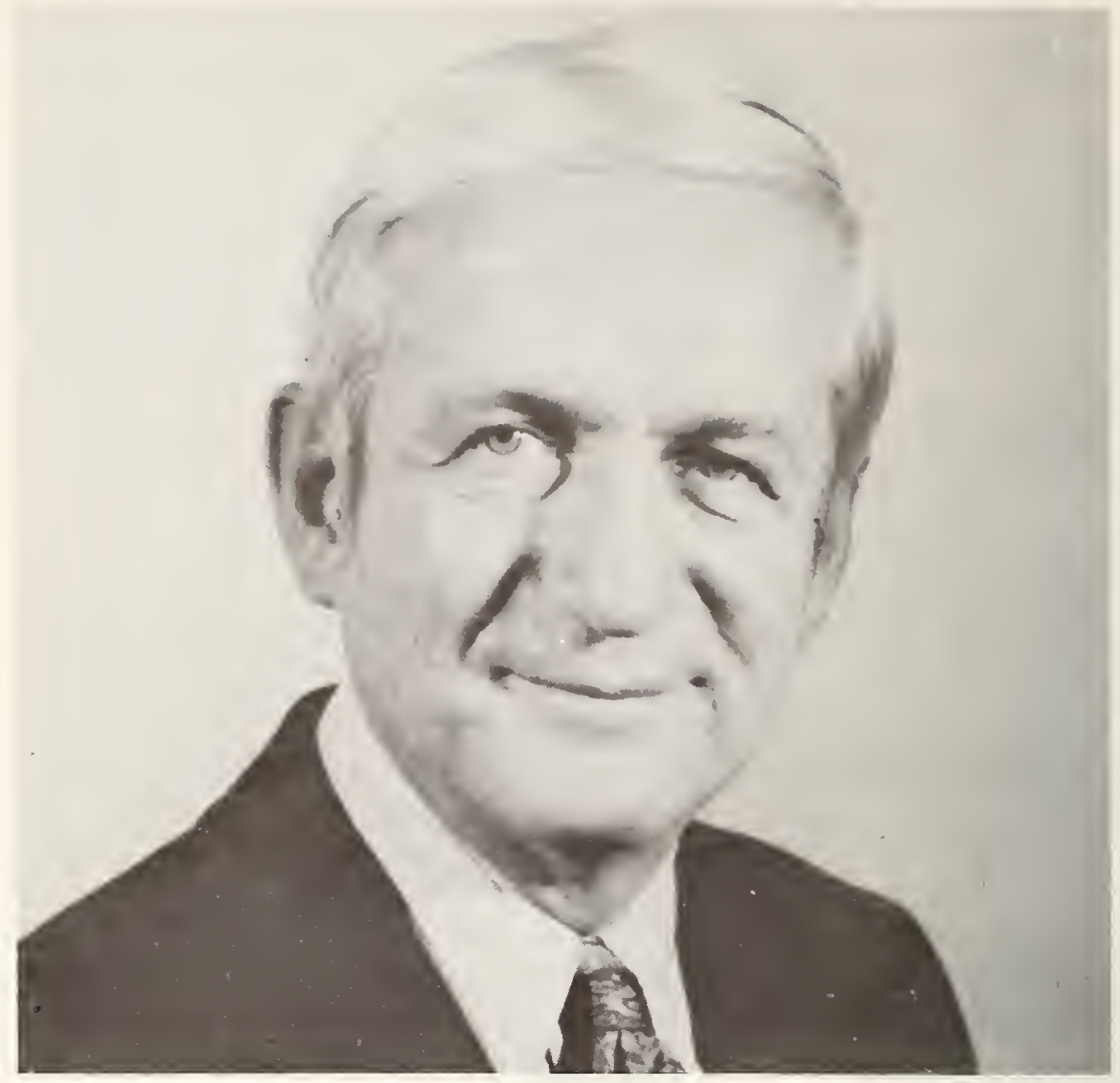

Professor Norman F. Ramsey is Higgins Professor of Physics at Harvard University. As a distinguished physicist, his work has ranged from molecular beams to particle physics, with a concentration on precision measurements of electric and magnetic properties of nucleons, nuclei, atoms, and molecules. He and his associates discovered the electric quadrupole moment of the deuteron, proposed the first successful theory of the chemical shift for the magnetic shielding of nuclei in NMR, and developed high precision methods of molecular beam spectroscopy including the atomic hydrogen maser. He is the recipient of the Lawrence Award, the Davis and Germer Prize, and the Presidential Certificate of Merit. Professor Ramsey was formerly chairman of the Physics Department of the Brookhaven National Laboratory. In 1973 he was the Eastman Professor at Oxford University. Since 1966 he has been President of the Universities Research Association which operates the Fermi National Accelerator Laboratory. 


\section{QUANTUM SCIENCE AND ITS IMPACT ON METROLOGY}

\section{Introduction}

This series of lectures celebrates both the 200th anniversary of the United States and the 75th anniversary of the National Bureau of Standards. However, in view of my subject, I should call attention to the fact that we are also celebrating the 75th anniversary of quantum science. In his great 1901 paper on blackbody radiation, Max Planck introduced the very first quantum ideas and the now famous Planck constant $h$. It is appropriate indeed that the National Bureau of Standards and quantum science should have started in the same year since that institution and that science have each had great impact on metrology, the science of accurate measurement.

During the 75 years of its existence quantum mechanics has become the basis of our interpretation of the physical properties of all normal matter. Even classical mechanics achieves its validity only insofar as it provides a satisfactory approximation to the proper quantum mechanical description. From this point of view quantum science impacts all measurements and my assigned topic of Quantum Science and Its Impact on Metrology becomes so all-encompassing that it virtually disappears.

However, it is possible to identify certain characteristic features of quantum mechanics that have profound implications on the science of measurement and to concentrate attention on these characteristics. This is the procedure I shall follow but I must emphasize that a short list of such features is necessarily arbitrary and incomplete.

\section{Characteristic Quantum Features}

Four quantum features with profound effects on the science of measurement as listed in figure 1 are:

1. Discrete quantum states

2. Identity principle

3. Heisenberg uncertainty principle

4. Wave and coherent phase phenomena

A quantum mechanical system can exist in certain specific energy states $W_{1}, W_{2}, \ldots W_{n}$ and in many cases these energy states are discrete. When such a system makes a transition between one such discrete state 
1. DISCRETE QUANTUM STATES

2. IDENTITY PRINCIPLE

3. HEISENBERG UNCERTAINTY PRINCIPLE

4. WAVE AND COHERENT PHASE PHENOMENA

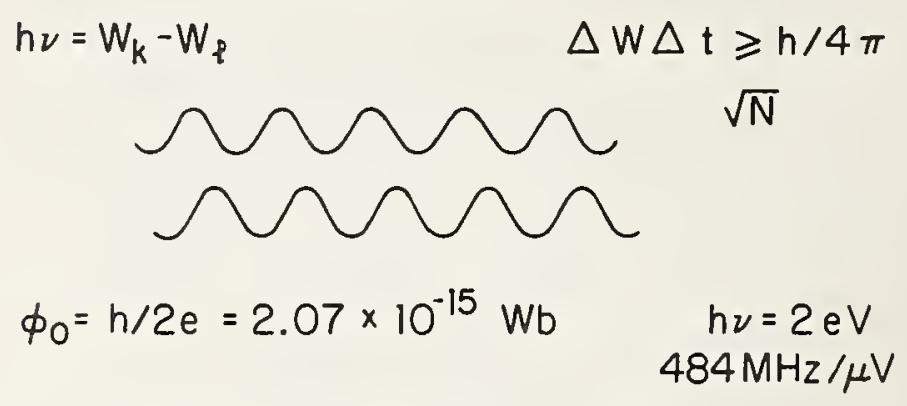

Figure 1. Characteristic quantum features.

and another, energy is conserved. For example, if a photon of energy $h \nu$ is emitted in such a transition

$$
h \nu=W_{n}-W_{l}
$$

where $h$ is Planck's constant and $\nu$ is the frequency of the emitted radiation. The existence of such discrete frequencies permits accurate measurements and accurate frequency standards that simply would not be possible if the allowed frequencies had the continuous distribution that charac. terizes a classical system.

The meaning of identity of two particles in quantum mechanics is far more profound than the normal meaning of the word identical. I am the father of identical twin daughters, and indeed they are remarkably alike. Nevertheless, when one speaks of two protons or two hydrogen atoms as being identical much more is meant. For example, one can test the identity of two particles by scattering one from another in which case there is a marked difference in the observable scattering if the particles are truly identical instead of being almost identical. The identity principle has a profound effect on the science of measurement. It provides, for example, assurance that the frequency of a cesium atomic beam frequency standard will be independent of whether the cesium comes from Britain or the United States. The identity principle also adds much to the significance and universality of accurate physical measurements. With the quantum assurance that all closely similar atoms are indeed identical, a scientist mea. suring an atomic property on the earth knows that the result of his mea- 
surement will be applicable to a scientist on the moon as well. This consequence of the identity principle is in sharp contrast to geology; an accurate geological measurement on the earth does little to determine the geology of the moon. This difference in the two sciences is due to the lack of an identity principle in geology.

A guiding principle in the early development of quantum mechanics was the Heisenberg uncertainty principle which correctly emphasizes certain quantum limitations on precision measurements. For example, if the energy $W$ of a system is measured to an accuracy $\Delta W$ then there must be an uncertainty $\Delta t$ as to the length of time it is in the energy state $W$, where

$$
\Delta W \Delta t \geqslant h / 4 \pi
$$

Although the uncertainty principle indeed limits the accuracy to which two complementary variables can be measured, Planck's constant $h$ is fortunately small compared to macroscopic angular momenta, so remarkably accurate measurements can be made. The world of precision measurement would be far different if $h$ were $6.6 \mathrm{~J}$ instead of $6.6 \times 10^{-34} \mathrm{~J}$ (the world itself would, of course, also be far different in that case).

The limitations of the Heisenberg uncertainty principle are further diminished by their applying to a single measurement on a single system. If $N$ different systems are measured, the uncertainty in principle is reduced by $\sqrt{ } N$. For example, within less than 10 seconds we can easily detect a shift of one part in $10^{14}$ in a $10^{9} \mathrm{~Hz}$ hydrogen maser oscillator even though the Heisenberg uncertainty limit is $0.1 \mathrm{~Hz}$ or one part in $10^{10}$. The reason we can do so much better is that within the 10 seconds we are mak ing measurements on $10^{13}$ atoms so the effective Heisenberg uncertainty limit in principle is $3 \times 10^{6}$ lower than that for a single atom.

The wave nature of the quantum mechanical state function and the possibilities of coherent effects with such coherent waves provide many different devices for precision measurements including interferometers, masers and lasers. Furthermore, the energy gap that results from electron pairing in superconductors provides coherency effects on a macroscopic scale. The coherent waves of electron pairs, for example, give rise to the remarkable phenomenon of zero electrical resistance in a superconductor. As a further consequence of such coherency on a macroscopic scale, the magnetic flux enclosed by a superconducting ring must be limited to an integral multiple of the flux quantum

$$
\phi_{0}=h / 2 e=2.07 \times 10^{-15} \mathrm{~Wb} .
$$

The same coherency on a macroscopic scale gives rise to the remarkable Josephson effects when a thin barrier, through which the electron pairs can quantum mechanically tunnel, is placed between two superconductors. If, for example, there is a voltage $V$ across such a Josephson junction the electron pairs will change energy by $2 \mathrm{eV}$ in crossing the junction and the 
current through the junction will be accompanied by an oscillatory current whose frequency satisfies the usual quantum relation of eq (l) so

$$
h \nu=2 \mathrm{eV}
$$

As far as is known at present this relation is exact and permits accurate measurements of voltages in terms of frequencies. The factor relating frequency to voltage is approximately 484 megahertz per microvolt.

If we now look back at the four characteristic quantum features we see that only one - the Heisenberg uncertainty principle-provides a limitation to the accuracy of measurement and that this limitation in most instances is so low as not to be serious. The other three characteristics give universality and significance to accurate measurements or else permit new high precision quantum measuring techniques. As a result in our quantum world, we can make meaningful measurements that are orders of magnitude more accurate than would be possible in a purely classical world. This was vividly brought to my attention by my difficulty in having a slide made on a recent visit to England. The slide of concern is shown in figure 2. For the present purpose we need not concern ourselves with the content of the slide-it lists some properties of atomic hydrogen that have been accurately measured with hydrogen masers developed at our laboratory. The engineer at Oxford who was asked to prepare the slide at first refused on the grounds that he had been trained as an engineer and from the point of view of an engineer it was utterly meaningless to express any physical number with such ridiculously high accuracy. From the point of view of a

$$
\begin{gathered}
\text { ATOMIC }{ }^{1} H,{ }^{2} \mathrm{D}, \text { and }{ }^{3} \mathrm{~T} \\
\Delta \nu_{H}=1,420,405,751.7680 \pm 0.0015 \mathrm{~Hz} \\
\Delta \nu_{D}=1,327,384,352.5222 \pm 0.0017 \mathrm{~Hz} \\
\Delta \nu_{T}=1,516,701,470.7919 \pm 0.0071 \mathrm{~Hz} \\
\mu_{D}=0.00152103221(2) \\
\text { Bohr magnetons } \\
\mu_{J}(H) / \mu_{J}(D)=1.00000000722(10) \\
\mu_{J}(H) / \mu_{J}(T)=1.0000000107(20)
\end{gathered}
$$

Figure 2. Hyperfine separations, proton magnetic moment and atomic moment ratios of atomic hydrogen, deuterium and tritium. 
classical engineer he was quite right. To express the height of the Washington Monument to an accuracy of one part in $10^{13}$ would be utterly meaningless - its height can't even be defined to that accuracy and even if it could it would change by much more than that amount from second to second as it blew in the wind and underwent thermal expansion and contraction. However, in a quantized world such accurate measurements are both meaningful and possible.

\section{Applications to Measurements}

Now that we have discussed the aspects of quantum science that have the greatest impact on measurement, I would like to conclude my lecture with a few illustrations of the way these quantum characteristics can be utilized in accurate measurements. For the reasons discussed earlier, applications occur in almost all fields of measurement. For example, one of the earliest such applications was to the use of interferometers in the measurement of length and in the definition of the unit of length in terms of the wavelength of the radiation emitted when a krypton atom makes a transition between two of its quantized states. Some of the other applications are listed in figure 3.

However, to avoid an endless list of precision measurement techniques, I shall limit further discussion of the illustrations to just two of the fields: (a) Measurement of time and frequency and (b) Measurements dependent on the macroscopic coherent quantum phenomena of superconductivity.

\section{APPLICATIONS}

LENGTH

SPEED OF LIGHT

MAGNETIC FIELD

$X$-RAY INTERFEROMETRY

RYDBERG CONSTANT

PARTICLE MAGNETIC MOMENTS
AVOGADROS CONSTANT

THERMOMETRY

FINE AND HYPERFINE STRUCTURE

TIME AND FREQUENCY

SUPERCONDUCTIVITY AND JOSEPHSON EFFECTS

Figure 3. Measurements affected by quantum science. 
The fundamental unit of proper time and frequency is now defined in terms of the frequency emitted in accordance with eq (1) when an atom of cesium in an atomic beam makes a transition from one designated quantum state to another with the states being such that in one the electron spin and magnetic moment are parallel to the nuclear spin while in the other they are oppositely oriented. With such a cesium atomic beam standard, the Boulder Laboratory of the National Bureau of Standards and other laboratories define and measure time and frequency to an accuracy of a few parts in $10^{13}$. In contrast, a few decades ago, the unit of time was defined in terms of the motion of the earth around the sun, which fluctuated from year to year by more than one part in $10^{9}$.

For measurements requiring great stability, the hydrogen maser is often used. Like the cesium beam tube, the hydrogen maser frequency depends on transitions between two designated quantized atomic states but in addition the maser measurement of the frequency depends on quantum mechanical coherency effects and in particular on the coherent stimulated emission of the radiation emitted in the transition.

Although high precision quantum measurements of time and frequency have been used for a number of years the field is still a very active one with a diversity of new ideas both for achieving increased precision and for extending the range of frequencies which can be measured with high precision. Some of the present and prospective devices for frequency and time standards are listed in figure 4. Almost all of these devices depend on measurements of relatively isolated atoms or molecules. Consequently, the atoms or molecules are in thermal motion and are subject to both first order and second order Doppler shifts. The second order Doppler shift is just the time dilation of a moving clock in special relativity (the often discussed phenomenon that a rapidly moving clock runs slow) and is proportional to $(v / c)^{2} \sim 10^{13}$. This is sufficiently small that many current frequency standards do not eliminate the shift but merely provide means for estimating the magnitude of the correction. On the other hand, the first order Doppler shift - the familiar increase in the frequency received from an approaching radiation source-is proportional to $v / c \sim 3 \times 10^{-7}$ so any competitive frequency standard must provide a means for eliminating the first order Doppler shift. Consequently, the essential features of the different frequency standards can most simply be given by describing the way that each one eliminates both frequency shifts and resonance broadening from first order Doppler shifts.

In the cesium and molecular beam devices the first order Doppler shift is eliminated by the use of two separated oscillatory fields of coherent radiation of the same phase. In the hydrogen maser, the first order Doppler shift is eliminated by confining the hydrogen atoms to a small volume 


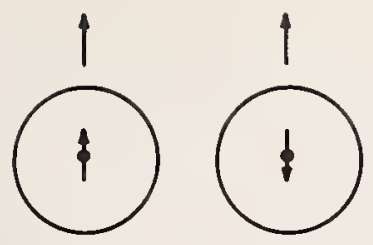

FIRST ORDER DOPPLER

SECOND ORDER DOPPLER

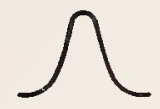

\section{Cesium Beom}

Molecular Beam

Hydrogen Maser

Trapped Ion Spectroscopy

Laser Saturated Molecular Absorption

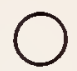

Double Resonance Spectroscopy

Two Photon Spectroscopy

Superconducting Cavities

Laser Resonance Cooling

Figure 4. Experimental methods applicable to time and frequency measurements.

which is traversed many times during the radiation process of each atom so the velocity averages to zero. In trapped ion spectroscopy, the first order Doppler shift is eliminated for the same reason. With laser saturated molecular absorption devices, laser light is passed in opposite directions through, say, a $\mathrm{CH}_{4}$ absorption cell and there is a minimum of the absorption at a frequency corresponding to no Doppler shift since the stationary molecules absorbing at that frequency absorb the light from both directions equally well and hence are more readily saturated than are the moving molecules which respond at most to the light from a single direction. Similarly, both double resonance spectroscopy and two photon spectroscopy require the absorption of two photons and, if these photons come from the opposite directions, the Doppler shifts would prevent simultaneous absorption of both photons except for the absorption by molecules for which the Doppler shifts are zero, i.e. for molecules with a negligibly small component of velocity along the direction of propagation of the radiation. A carefully designed, superconducting tuned cavity has high frequency stability for periods of time up to 1000 seconds and of course no Doppler shift since its active element is stationary. Although such a device is not suitable as an absolute time standard, it is of value when a powerful oscillatory signal is required with high stability over relatively short intervals of time.

The active constituents of most potential frequency standards are relatively free atoms, molecules or ions and even if the first order Doppler shift is eliminated there may remain the above second order Doppler shifts 
of magnitude $10^{-13}$. If progress is to be made much beyond the present $10^{-13}$ accuracy, means must be found for reducing the magnitude of the second order Doppler shift. Even the devices which eliminate the first order Doppler shift by being insensitive to molecules with a nonvanishing longitudinal velocity component are subject to second order Doppler shifts from the transverse velocity components unless special steps are taken to eliminate the transverse components, say by the use of a well-defined atomic beam, which greatly diminishes the intensity. A new possible technique for reducing the magnitudes of the velocities has been proposed by Schawlow and Hansch and by Fortson and Dehmelt but the proposals are so far untested. The basic idea is to cool, say, atoms by shining on them light whose frequency is slightly below an allowed absorption frequency by an amount such that absorption occurs only through the first order Doppler shift of the approaching atom. However, when the cxcited atom spontaneously radiates the radiation will be emitted in all directions and consequently, on the average, the radiation will be of higher energy than the absorbed light and this additional energy must come from the kinetic energy of the atom. In this fashion atoms, molecules or trapped ions can be cooled by many successive absorptions and reemissions. It will be of great interest during the coming years to see if these techniques for reducing the second order Doppler shift are successful and to see if they lead to a marked increase in the accuracy of clocks and frequency standards.

\section{Superconductivity and Josephson Effects}

The last category of quantum phenomena with a great impact on measurement is that associated with the macroscopic coherent quantum phenomena of superconductivity. Some applications arise directly from the zero resistance characteristic of superconductivity. An illustration of this was the previously discussed use of superconducting tuned cavities in frequency measurements. Another application is the use of superconductors as magnetic shields where the magnetic flux quantization with superconductors, discussed above, makes it possible to provide a perfect magnetic shield.

However, the most spectacular applications of macroscopic coherent quantum effects to accurate measurements have been with the previously mentioned Josephson junctions that result from separating two superconductors by a thin barrier through which electron pairs can tunnel. Such a Josephson junction is shown schematically in figure 5. Several interesting effects occur at such junctions. One of these, known as the d.c. Josephson effect, is the passage of low currents through the junction with the potential $V$ across the junction remaining at $V=0$. However, as discussed earlier, for currents higher than a critical current $I_{c}$, a voltage difference $V$ appears 

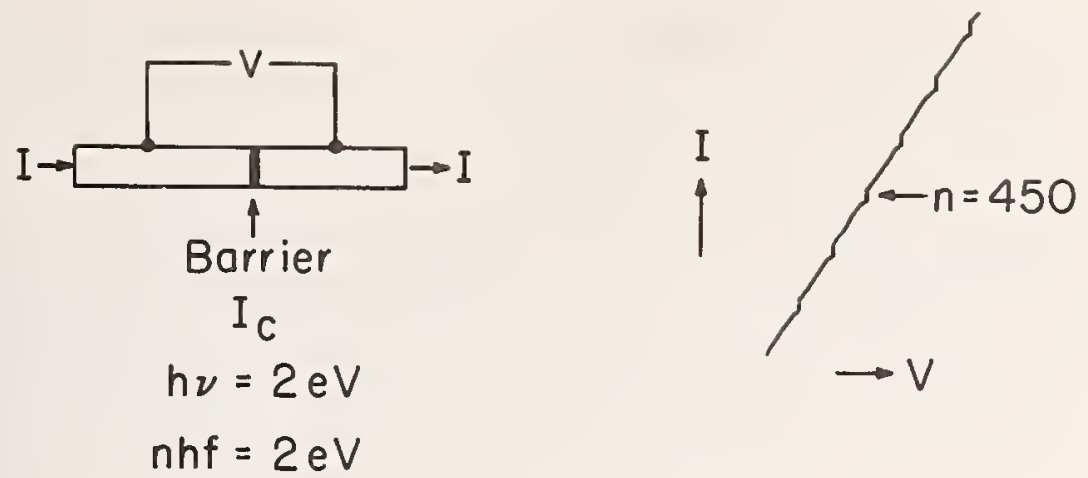

Figure 5. Josephson junction and Josephson effects.

across the junction but the passage of electron pairs between opposite sides of the barrier with a $2 \mathrm{eV}$ change in energy is accompanied by an oscillatory current (a.c. Josephson effect) whose frequency satisfies the usual quantum relation of eq (1) or eq (4) above. Finally, if microwaves of frequency $f$ are coupled to a Josephson junction, the current-voltage curve is modified by the appearance of steps as in figure 5 . The steps correspond to the stimulated emission or absorption of $n$ quanta of energy $h f$ by the strong microwave signal in the nonlinear Josephson junction while electron pairs cross the barrier with an energy difference of $2 \mathrm{eV}$. Consequently at each step

$$
n h f=2 e\left|\mathrm{~V}_{n}\right|
$$

Accurate measurements of the frequencies and associated voltage steps in eq (5) have provided an excellent determination of the fundamental quantity $2 e / h$. When this result is combined with other known measurements, it provides an accurate value for the fundamental dimensionless fine structure constant $\alpha=e^{2} / h c$. Inversely the Josephson effect and eq (5) can be used to measure d.c. volt age and provide an excellent basis for a definition of the volt.

The voltage to frequency conversion provided by the Josephson effect through eq (5) makes possible low temperature thermometry by accurate measurements of the thermal noise generated by a resistor, $r$, which acts as the thermometer.

The Josephson effect can also be combined with the previously discussed phenomenon of flux quantization. This can be done by forming a superconducting loop with one or two Josephson junctions included in the circuit as in figure 6. Such a device is called a SQUID (Superconducting QUantum Interference Device). The phase of the superconducting state changes around the loop by an amount proportional to the magnetic flux linking the loop and various measurable quantities become periodic in the' 

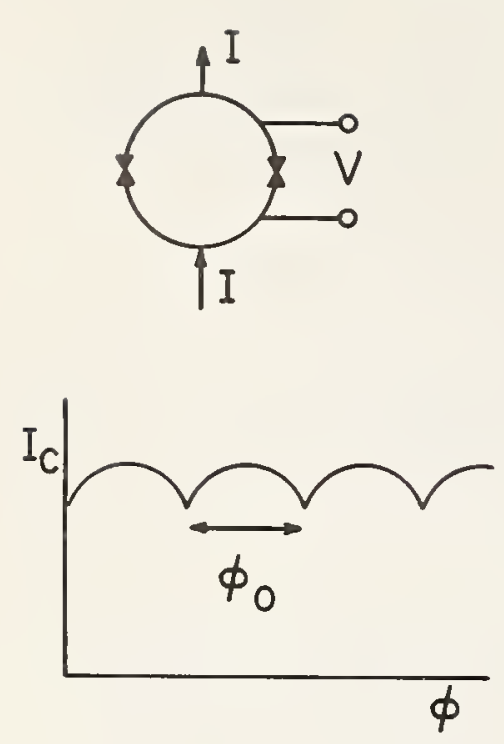

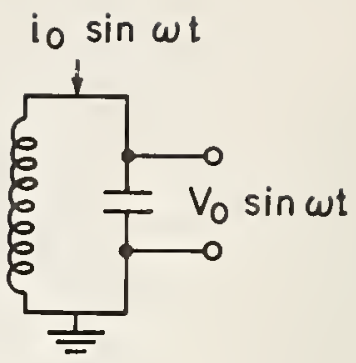

$$
\phi_{0}=h / 2 e
$$

Figure 6. SQUIDs (Superconducting QUantum Interference Devices).

magnetic flux $\phi$ with the period being the flux quantum $\phi_{0}=h / 2 e$ of the previous eq (3). For example, in the d.c. SQUID on the left side of figure 6 , the critical current $I_{c}$ changes periodically with $\phi$ as shown in that figure. Likewise, the voltage across one of the Josephson junctions in the d.c. SQUID is periodic in $\phi$. Alternatively, in the rf SQUID shown on the right side of figure 6 , the ring is coupled to an $L C$ resonant circuit to which an $\mathrm{rf}$ current $i_{0} \sin \omega t$ is applied. The amplitude, $V_{0}$, of the volt age across the resonant circuit is then a periodic function of the flux applied to the SQUID with the period being the flux quantum $\phi_{0}$. The SQUID can be used in several ways to measure magnetic flux. One is to lock on a sharp minimum and feed back a known current in a loop to keep the flux constant, in which case the SQUID is a null detector in a feedback system. In this way SQUIDs have been effectively exploited to measure magnetic fields and to compare direct currents. SQUIDs have also been used effectively in thermometry and in the measurement of radiofrequency currents.

\section{Conclusion}

The science of measurement has undergone a major revolution in the past 75 years. This has largely been due to the exploitation of the various quantum effects which make such precise measurements both possible and meaningful. The magnitude of advances can best be illustrated by 
comparing a few of the values of the fundamental constants as listed in Birge's 1929 tables and those of the CODATA evaluation of 1973 as brought up to date in 1975. In this comparison I could not go back to 1901 since two of the quantities $\alpha$ and $h$, were unknown at that time while $e, m_{e}$ and $N_{A}$ were in error by 300 percent. The precision of these measurements has increased by a factor of 3000 in less than 50 years. Although many different technical advances and much hard work have contributed to the increased precision, it is clear that most of the progress has been the result of new quantum techniques (fig. 7).

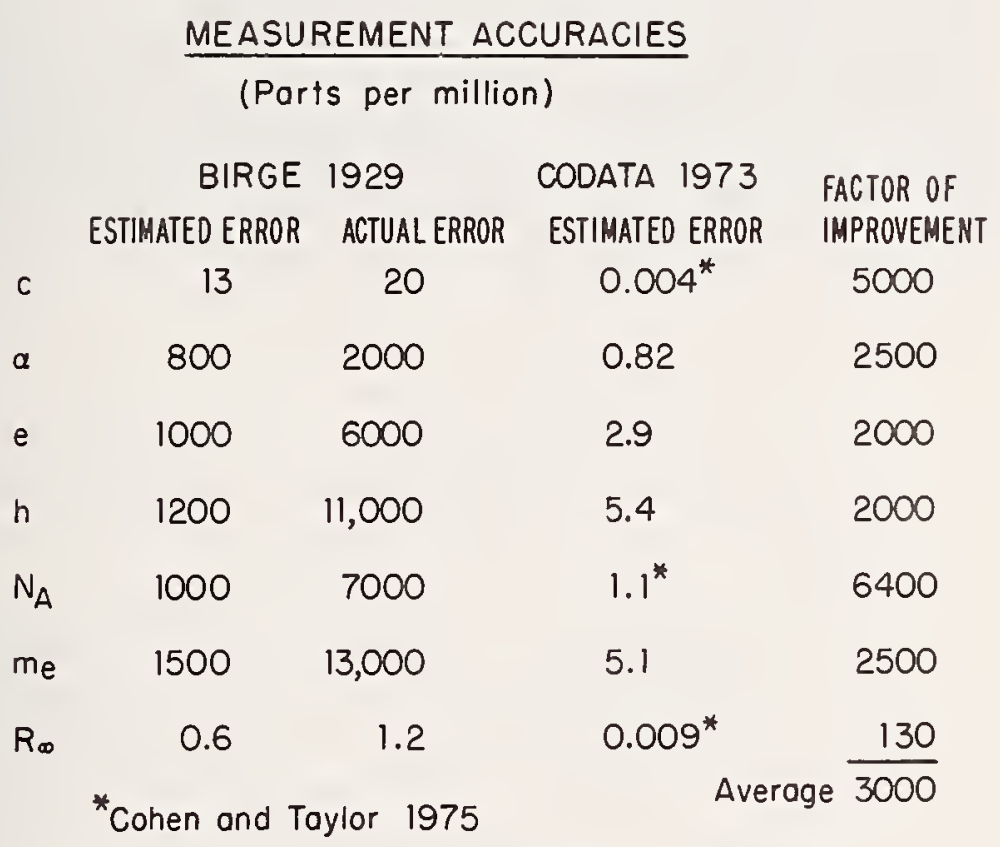

Figure 7. Measurement accuracies.

We thus see that the precision of basic measurements has increased more than a thousandfold during the past half-century. This progress in the science of measurement owes much to the existence of the National Bureau of Standards and to quantum science, each of which had its independent beginning a mere 75 years ago. It will be a formidable task for scientists to match these achievements during the next three-quarters of a century. 


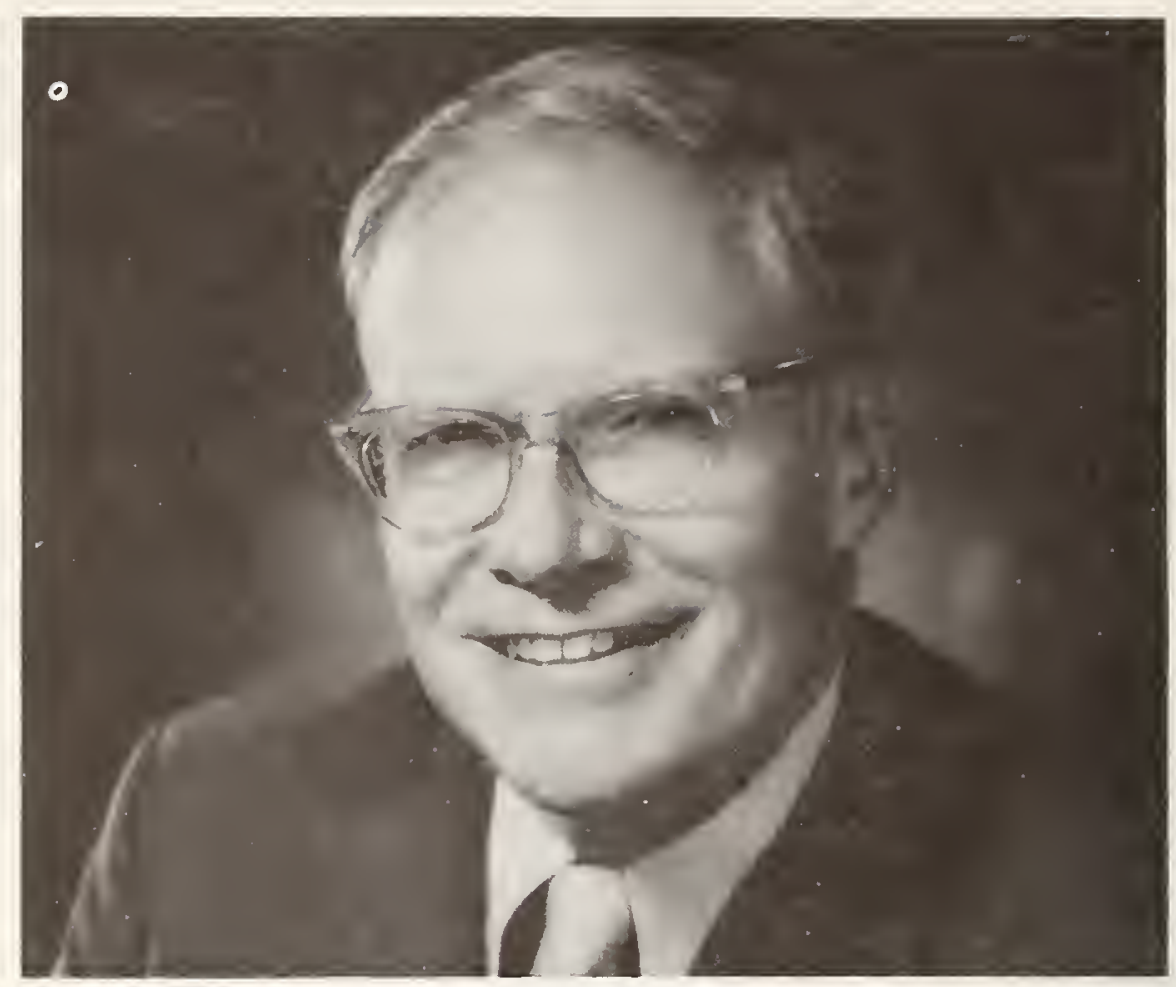

Dr. William O. Baker is President of Bell Laboratories. His science and technology work since 1939 has been on materials basic to telecommunications and electronics, with emphasis on the solid state properties of dielectrics and polymers. He also did pioneering work leading to the development of heat shields for missiles and satellites and the present use of polymer carbon composites. Dr. Baker has served in the last five Administrations in Washington in assignments linking Federal and independent science and technology activities. He is a member of the National Academy of Sciences, the National Academy of Engineering, the Institute of Medicine, and the American Philosophical Society. Dr. Baker was chairman of the President's Advisory Group on Anticipated Advances in Science and Technology and is Vice Chairman of the President's Committee on Science and Technology, a member of the National Cancer Advisory Board, and the Board of Higher Education of New Jersey. He is a past member of the National Council of Educational Research, the National Commission on Libraries and Information Science, and the National Science Board of the NSF, as well as a member of the President's Science Advisory Committee. He is trustee of Princeton, Carnegie-Mellon and Rockefeller Universities. He holds the Perkin Medal of the Society of Chemical Industry and the Priestley Medal of the American Chemical Society. He is the recipient of the Industrial Research Man of the Year Award, the Procter Prize, the Frederik Philips Award, American Institute of Chemists Gold Medal, the Edgar Marburg Medal (ASTM), and the ACS Charles Lathrop Parsons Award. 


\section{MATERIALS}

\section{Introduction}

I am honored to join in this series of meetings assessing the current state and future of science and technology in America on the occasion of the 75th Anniversary of one of the great scientific-technological institutions of our time. Although the admiration and respect of the national community of scientists and engineers for the National Bureau of Standards are widespread, they are especially strong in those of us who have come to know the NBS and its people intimately through years of collaboration in federal programs.

In my case, this began in the World War II years when my late as sociate, Dr. R. R. Williams, was designated to organize the U.S. research and development capability for synthetic rubber - one of the forerunners of the synthetic materials that now figure so prominently in our national life. The NBS Associate Director Dr. A. T. McPherson, Dr. L. Wood and others responded superbly to the task of helping to create a new materials industry and national capability. In this 36th year of unbroken affiliation with the staff and leadership of the NBS, I can report directly the unclouded record of integrity and excellence which that organization has provided in its national service.

During this span, it has been my privilege to see and have some part in the steadily growing resources of the NBS and its evolving structure, including particularly for our subject this morning the Institute for Materials Research. This section has properly caught the dynamic qualities of discovery and application of materials, which have been among the vigorous frontiers of the mid-20th century. Thus, in reviewing briefly some principal currents of thought and action in that field, we are also saluting the 75th Anniversary of the study of materials in the NBS, especially as symbolized by the spirited work of the present Institute for Materials Research and its Director, Dr. J. Hoffman.

\section{Regularity and Imperfection in Natural Materials}

Most of the matter comprising our planet solidifies as crystals, whose idealized forms are shown in figure 1. Interestingly, the major portion of textured matter has come from life processes, such as the oriented cellulose components in plants, the collagen in animals, the chitin in insects, 
SINGLE CRYSTAL

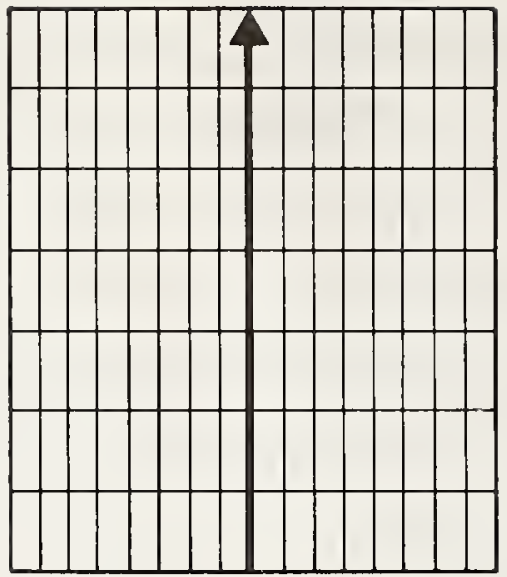

POLYCRYSTAL

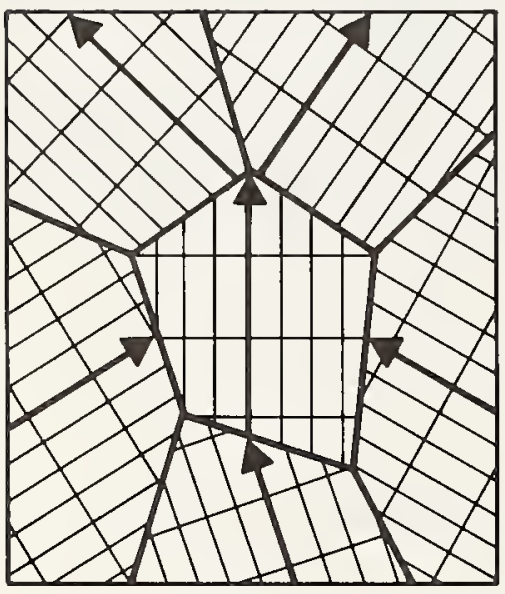

RANDOM

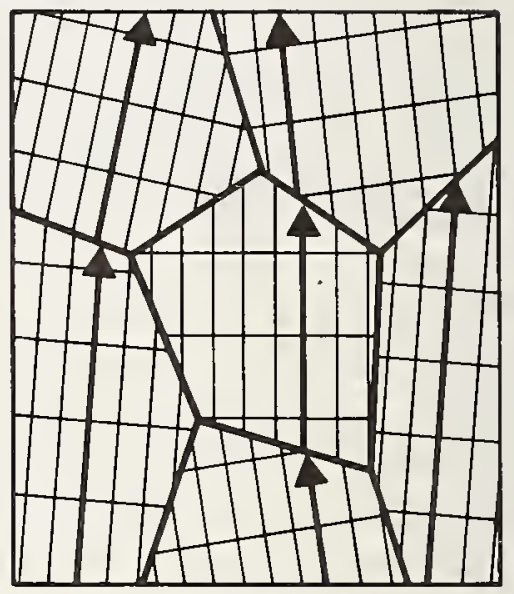

TEXTURED

Firure 1. Idealized crystal structures.

and the well-known keratins of silk fibers and the like. Of course, asbestos and similar mineral systems show some texturing as well.

One is charmed by the elegant regularity of atomic and molecular arrays in crystals. What a magnificent discipline seems to occur in nature, otherwise noted for its variety and disarray! But, although figure 2 indicates the atom's relatively frenzied process of finding an ordered crystalline lattice spot, and figure 3 even indicates how assemblies cooperate in yielding macroscopic crystals, nature has always had the last laugh. For the arrays are ever imperfect, with vacancies, dislocations and impurities, which only the most elegant techniques of the last few decades, particularly the zone refining of Pfann, have reduced to near ideal limits. Indeed, 


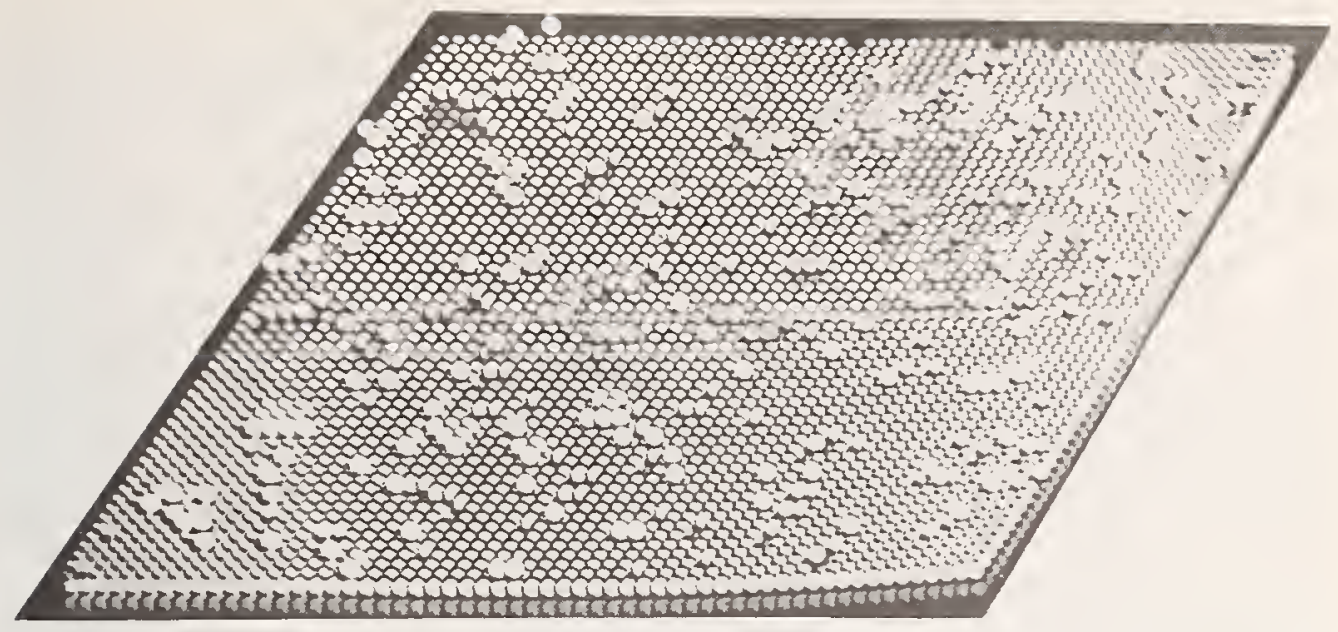

Figure 2. Computer model of crystal growth process: Atoms move randomly to fill incomplete lattice on surface of crystal.

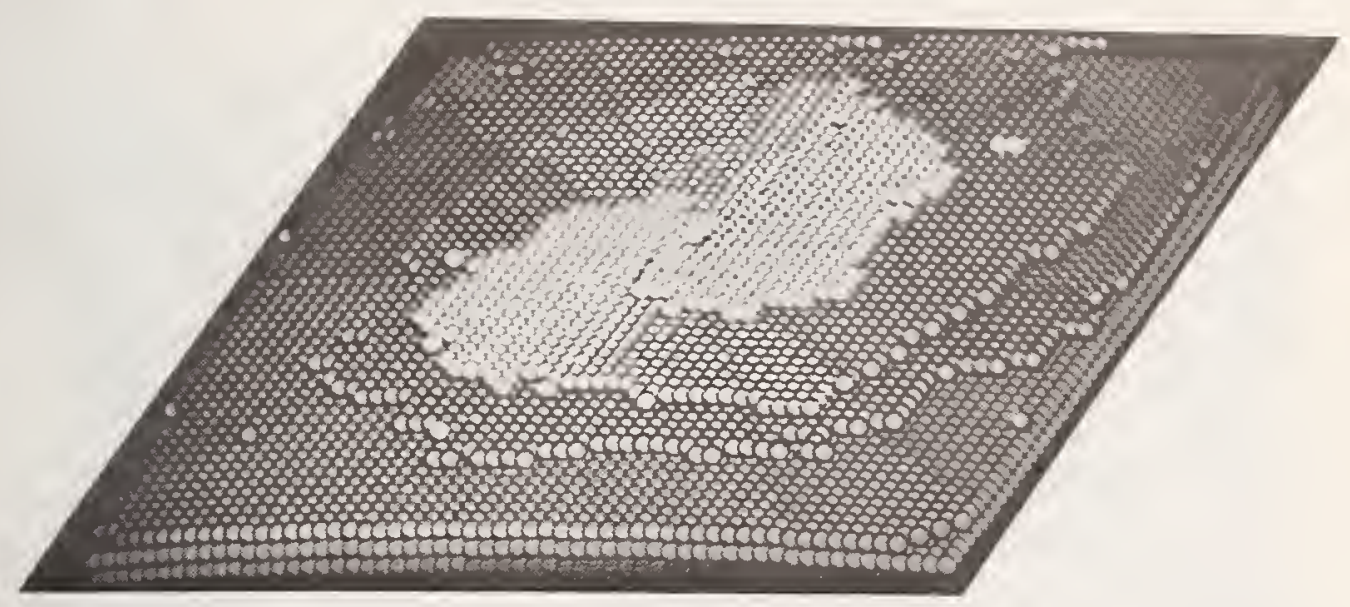

Fiqure 3. Computer model of spiral :rowth process in crystals: Vertical screw dislocation (in center of this model) acts as active growth site, perpetuating its spiral structure.

figure 4 depicts the original findings of Pfann and Vogel, confirming the theoretical suspicions of the early days of structural crystallography that there would be lines of defects, dislocations or vacancies in virtually every real crystal. The pits in figure 4 display that reality. And the curious adjustments to such reality made by thermally agitated atoms in crystals can in turn lead to bizarre recrystallization. One case is the famous and tenacious "whiskers," first discovered in tin by Galt and Herring some decades ago as spontaneous growth at room temperature. In figure 5, they appear in symmetrical fibrous form, since they are single crystals. 


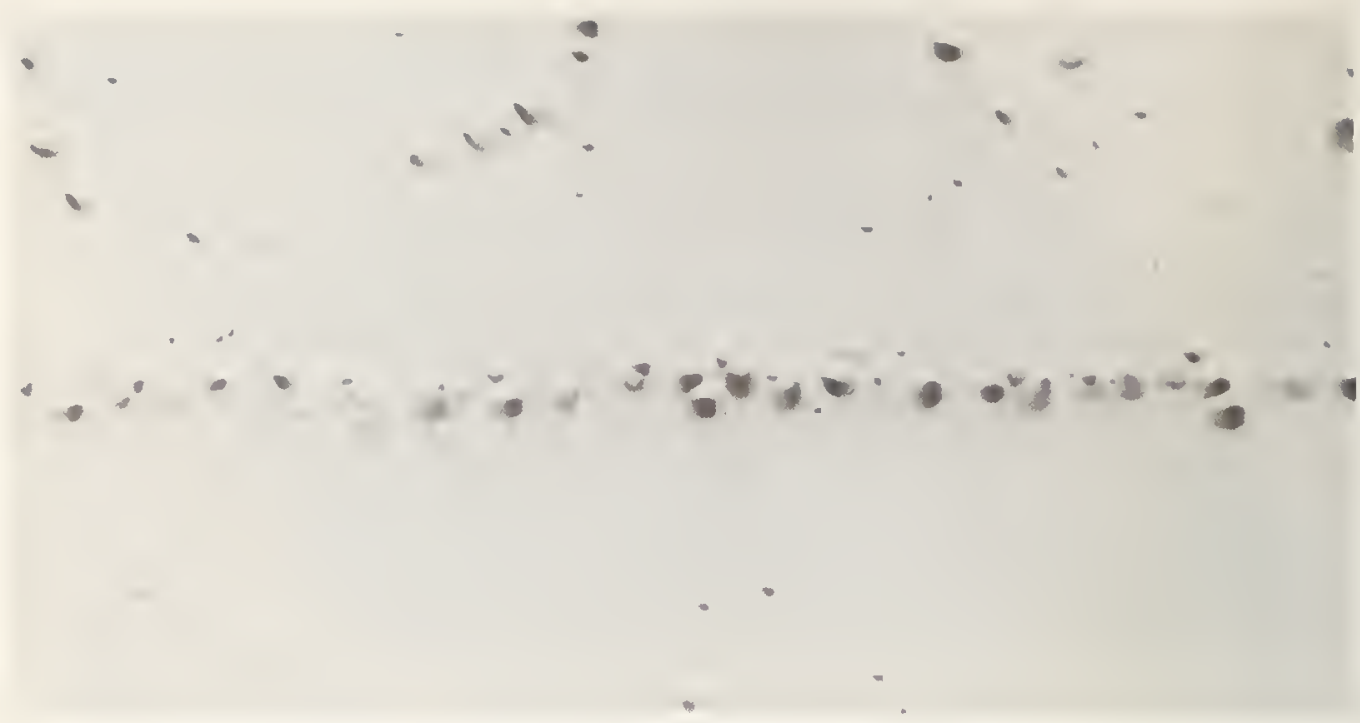

Figure 4. Single-crystal dislocations first discovered by Pfann and Vogel.

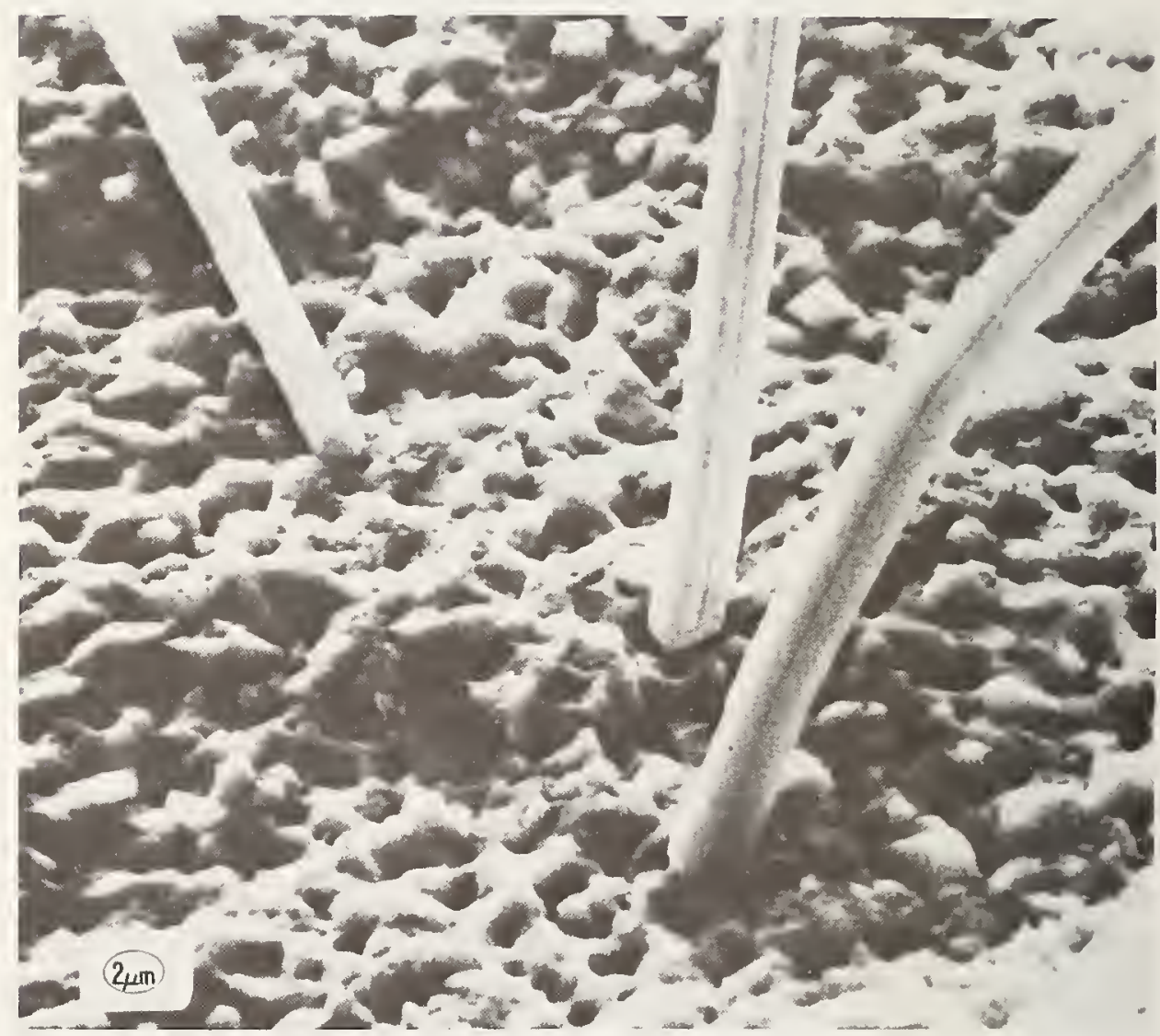

Figure 5. Fibrous, single crystal "whiskers"-microscopic, hair-like filaments-first discovered as a spontaneous growth on tin. Their unusual strength is due to near perfection of crystal structure. 


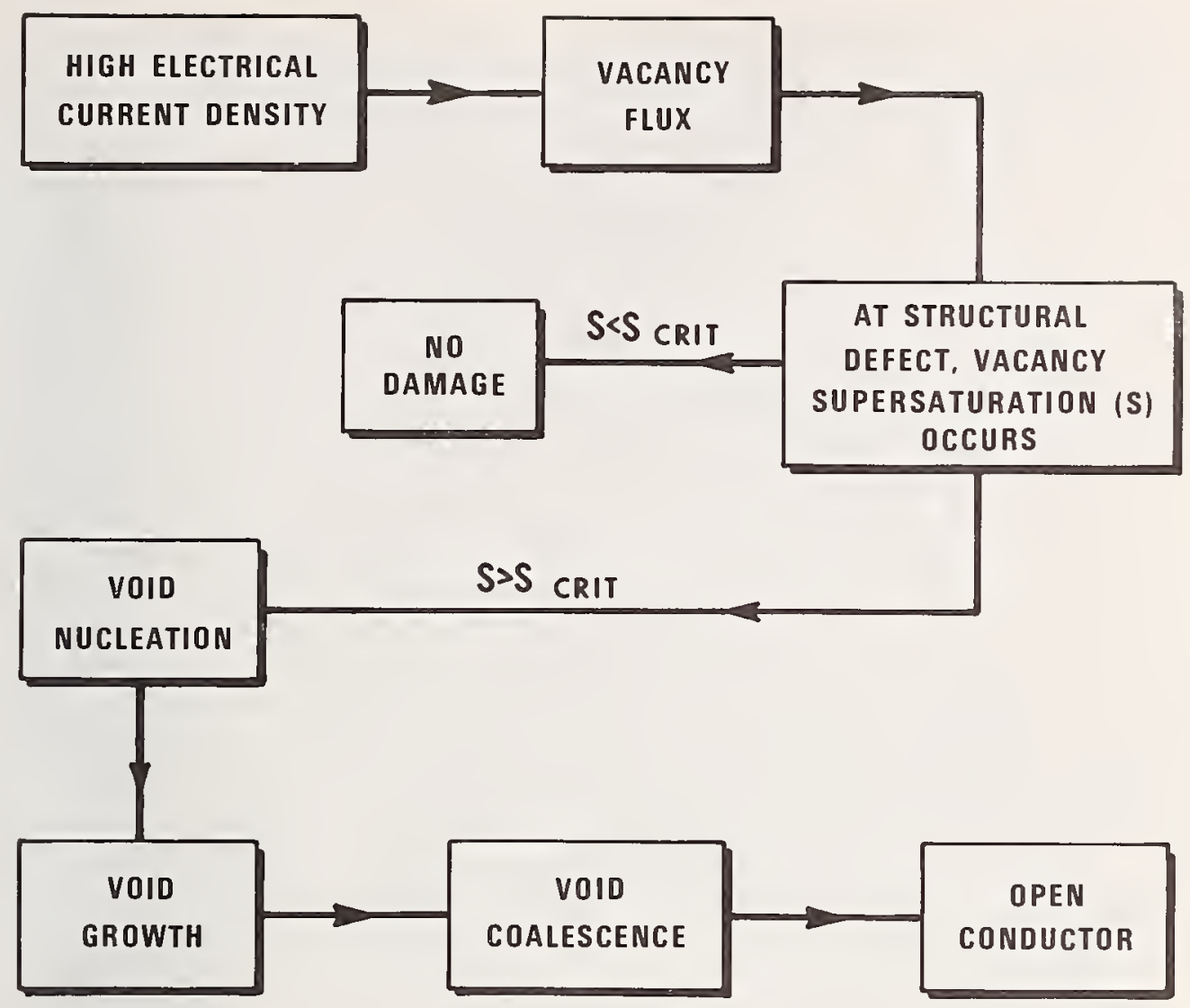

Figure 6. Electric potentials can cause the vacancies in thin films to coalesce, producing open circuits, fractures or physical discontinuities.

Further, we have found very recently that external fields, such as electrical potentials, have even more striking effects on the mobility of atoms and vacancies in the real surroundings of the crystal. To our amazement, vacancies are capable of aggregating in thin film structure, so important for modern electronics and for surface stabilization of metals and other systems! As diagrammed in figure 6, these aggregates can coalesce and cause open circuits, fractures or physical discontinuities in what are assumed to be atomically coherent layers of matter.

So already we see the future of materials research and development being shaped by properties unknown until the mid-century and, in many cases, recognized only in the past few years. For we know that the strength of matter, the youthfulness of steel, iron and copper, the hundreds of alloys comprising systems of which our civilization is constructed are determined by these imperfections and the movement of associated atoms in the solid. Just as the frailty of man may lie in spirit, so the frailty of matter lies in what isn't tangible, and in how it redistributes itself. Yet we know from these last decades that all the subtleties of strength, reliability and even reactivity are connected with these imperfections. 


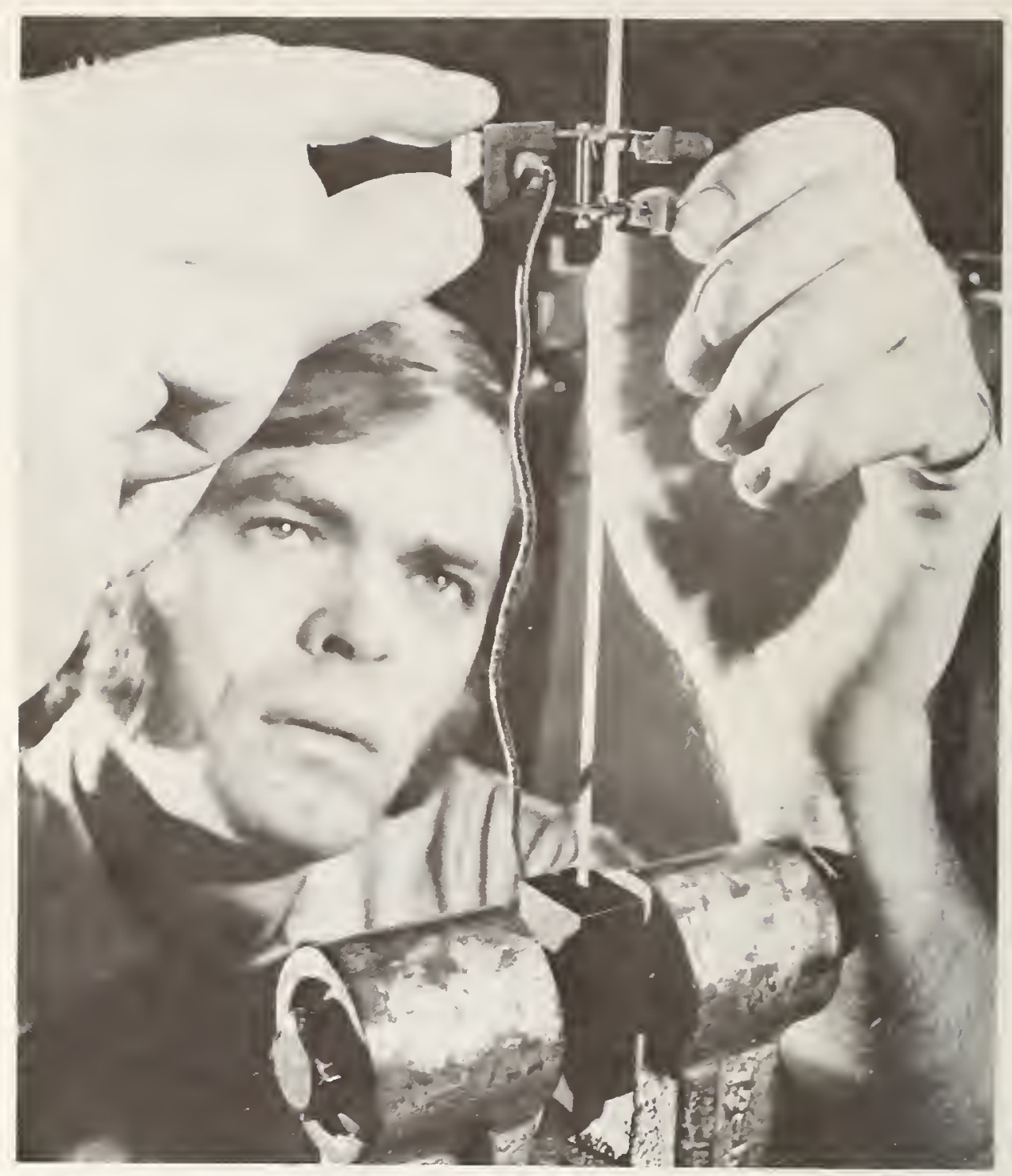

Figure 7. J. T. Plewes conducts tensile strength test of new copper-based spinodal alloy in which tin, uniformly distributed in a copper-based nickel matrix, impedes the movement of erystal dislocations.

The curious clicks that Mason and I "heard" ultrasonically years ago, as fractures were incited in stressed solids, have now been adapted as routine testing methods for determining the quality of ceramics, metals and various complex bodies.

But at the same time that we find the nonideal spanning the behavior of solids, as we have always known for liquids and gases. we find new opportunities for modulating it to enhance the usefulness of many classic materials. Thus, Plewes appears in figure 7 measuring the tenacity of the world's oldest refined metals, the bronzes and other copper alloys, after he has modified the susceptibility of their dislocations by spinodal decomposition of phases in the solid. This prevents large-scale dislocation move- 


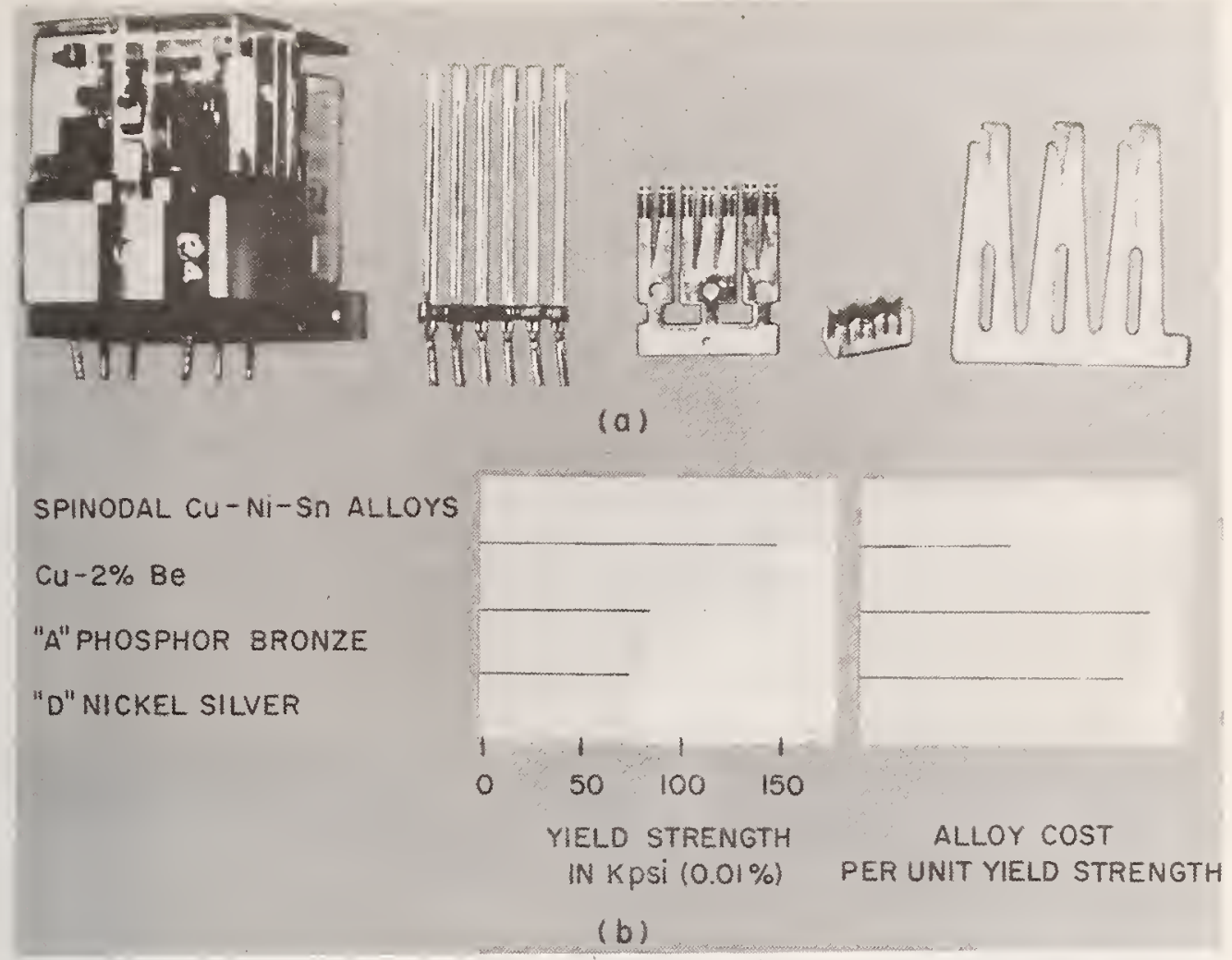

Figure 8. Spinodal copper alloys. (a) Typical applications. (b) Relative strength and cost.

TABLE 1. Yield strength of bronze

\begin{tabular}{lcc}
\hline \multicolumn{1}{c}{ MATERIAL } & $\begin{array}{c}\text { TYPICAL } \\
\text { YIELD STRENGTH } \\
\left(10^{3} \mathrm{pSi}\right)\end{array}$ & $\begin{array}{c}\text { ENHANCED } \\
\text { YIELD STRENGTH } \\
\left(10^{3} \mathrm{pSi}\right)\end{array}$ \\
\hline Cu-5Sn (PHOSPHOR BRONZE) & 70 & 110 TEXTURED \\
Cu-12Ni-28Zn (NCKEL SILVE) & 65 & $125 \quad "$ \\
Cu-9Ni-2Sn (MOD. CUPRONICKEL) & 45 & $105 \quad "$, \\
Cu-1.7Be (COPPER BERYLLIUM) & 145 & $170 \quad$, \\
Cu-9Ni-6Sn (MOD. CUPRONICKEL) & 50 & 150 SPINODAL \\
\hline
\end{tabular}

ments that cause most metals to yield and eventually break. Figure 8 shows the extraordinary improvements in the yield strength of copper alloys, whose qualities had already been improved for millennia, and whose yield stresses determined the course of civilization through the weaponry of the Bronze Age and much of the electrical media of the early 20th century.

Likewise in table 1 we see other profound improvements in the yield stresses of bronzes caused by computer-regulated texturing of the wire-like 
or planar systems involved. Here Dr. G. Chin has used the notions of crystal strength anisotropy, systematized by Professor G. I. Taylor in the early days of $x$-ray crystallography but not optimized, because of geometric complexity, until the computer analyses of the last few years.

But regulating the properties of matter, imperfect and complex as they may be, no longer depends on the amiable compliance of crystals dominated by one or two kinds of atoms. Rather, composition can create desirable new electronics as well as mechanics. Thus, although a brilliant era of magnetism, even electromagnetism, was signaled by the nickeldominated alloys of the permalloy-permendur era, we now find that subtly selected electronics of rare-earth shell structures, elements made as byproducts of refining processes in the nuclear energy age, yield new magnetic qualities. These may in the future revolutionize all magnetomechanical devices and systems such as armatures, miniature motors, etc. So, as illustrated in figure 9 , the unsurpassed magnetic strengths of the copper

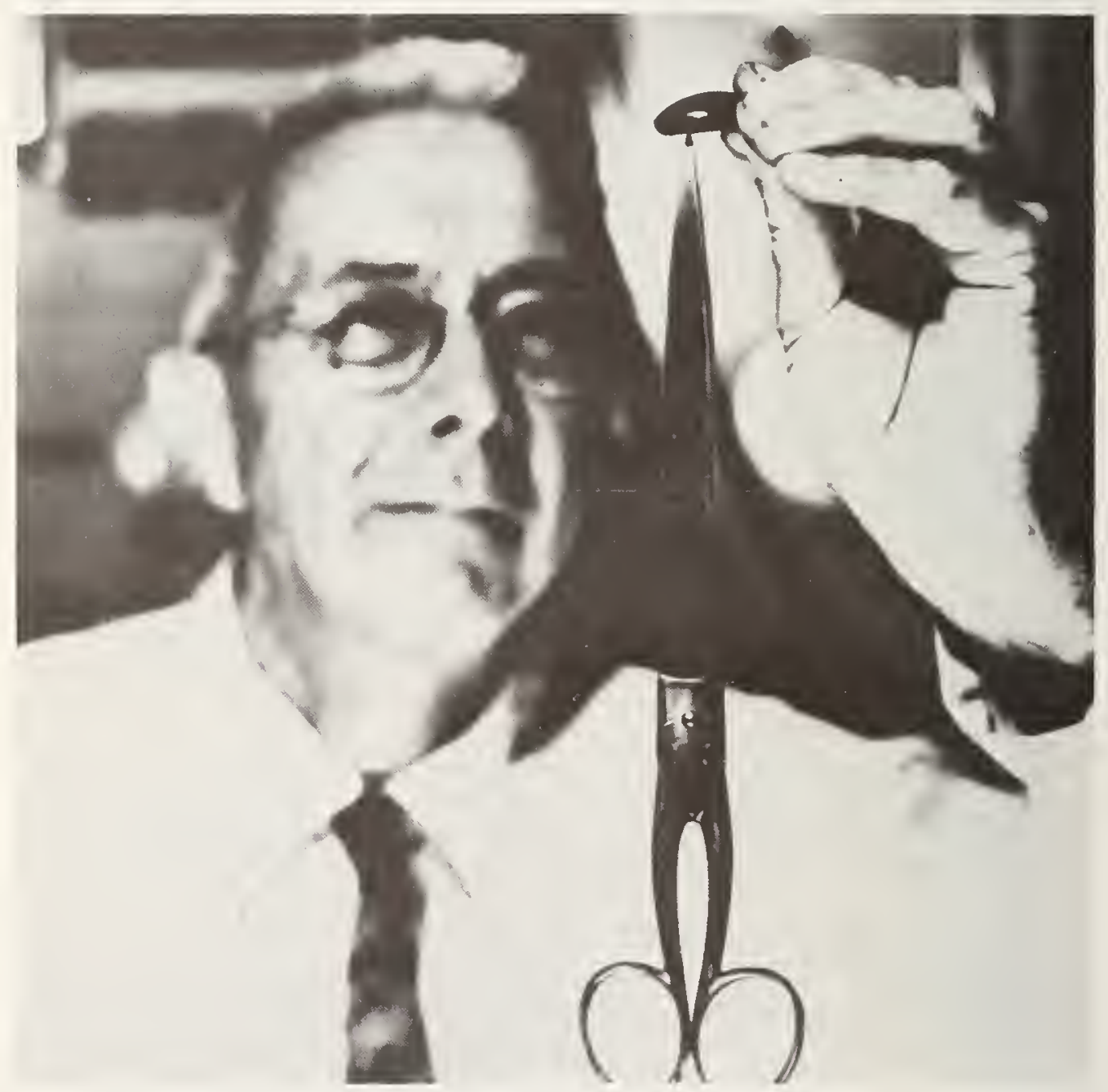

Fincre 9. E. A. Nesbitt "holding" scissors with rare-earth permanent magnet, $\mathrm{Co}_{3.3} \mathrm{Cu}_{1.2} \mathrm{Fe}_{0.5} \mathrm{Ce}_{0.25} \mathrm{Sm}_{0.75}$. 
rare-earth alloys represent yet another modification of crystal quality-its electronic and electromagnetic properties-characteristic of modern materials synthesis. Figure 10 depicts some of the magnetic field permeability qualities yielded by these compositions.

But if new magnetism is accessible, what about new conductivity? For it is said that the single most vital issue in the energy challenges of our industrialized society is to find more efficient electrical conductors for the distribution and use of centrally generated electricity. We all know that since the mid-century, when the subject of superconductivity was formally assessed as exhausted, new systems have been created with superconductivity exceeding any known before! Figure 11 shows critical properties of some high-transition superconductors. Recently, transition temperatures at the current highest level of $22.3 \mathrm{~K}$ have been reached by Matthias and his associates.

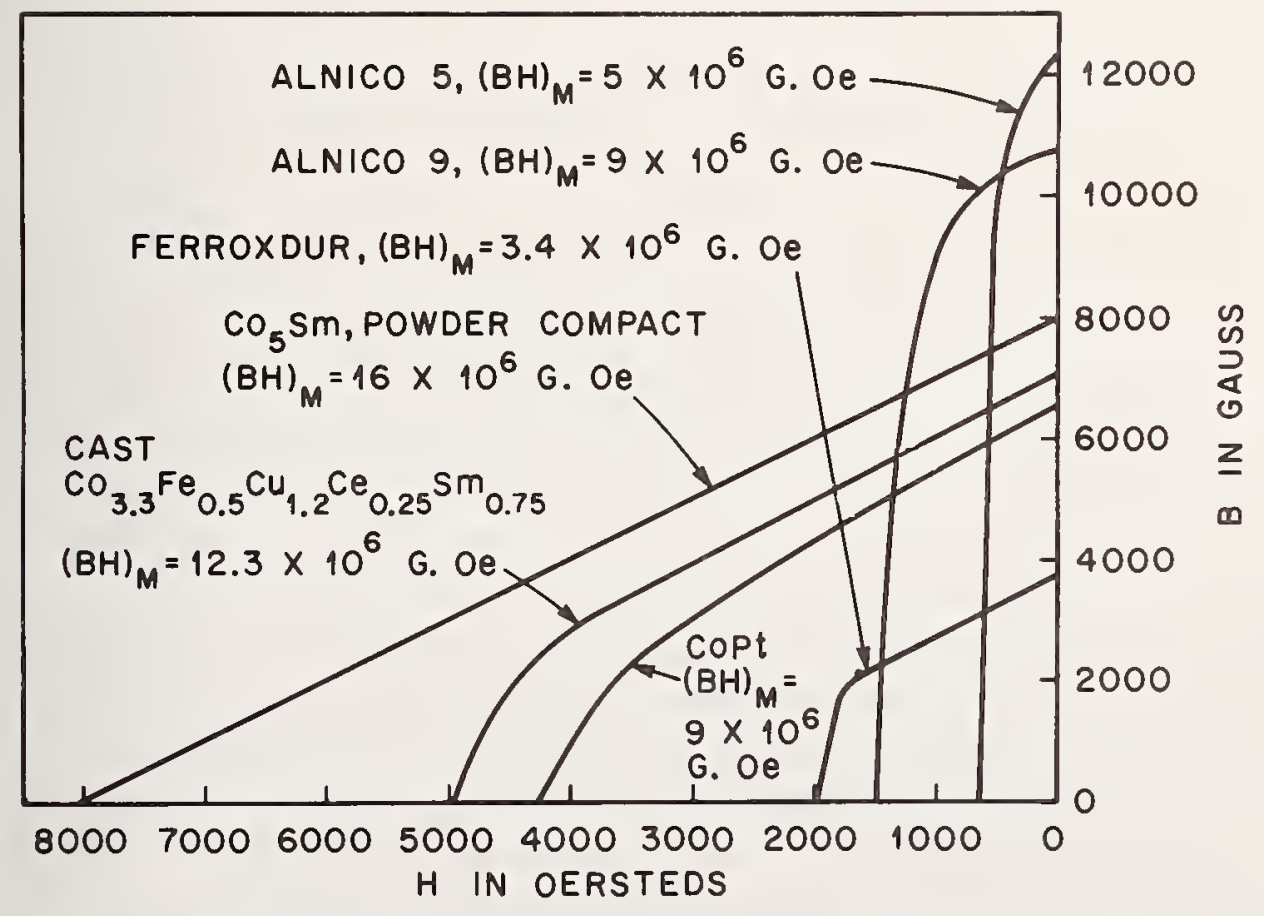

Figure 10. Magnetic-field permeability properties of copper rare-earth alloys. 


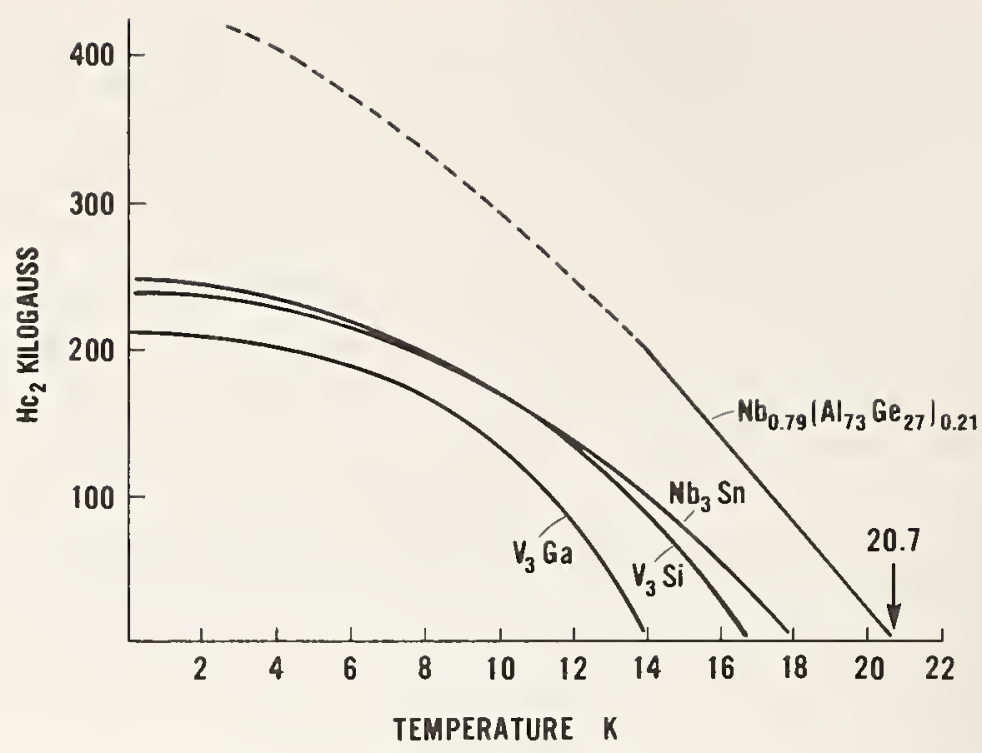

Figure 11. Critical field vs. temperature for several high-transition $\beta$-tungsten structure superconductors.

The question is, why can we not increase that temperature? Figure 12 shows that indeed we might, if we but knew how to introduce critical distorting forces into the lattice. Studies by Testardi and his associates, among others, have shown a curious consistent rise in transition temperature $T_{c}$ in a given system wherever there is appropriate distortion. It is as though the wonderful electron pairing theory, begun by Bardeen not long after his transistor invention, is realized and augmented by special perturbations of that same regularity whose unreality we saluted at the outset.

Well, it may be said, if all these wonderful things happen from crystal perturbation, and if the future of at least inorganic and metallic materials lies in distorting or otherwise disrupting the lattice still further, why not go all the way and make amorphous metal by sufficient quenching, as Duwez, Willens and their associates showed could be done some years ago? As 


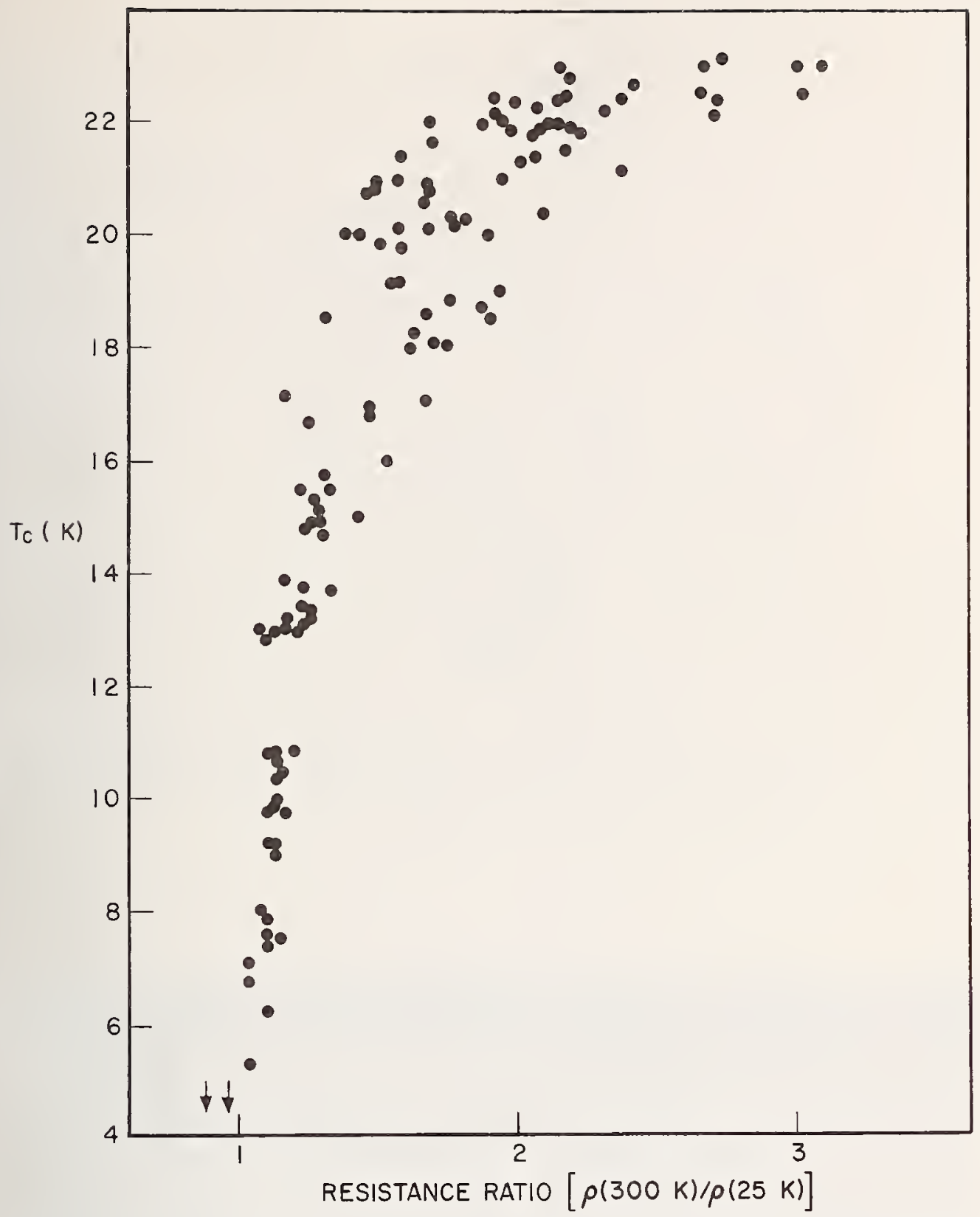

Figure 12. Relation between superconductivity transition temperature $\left(\mathrm{T}_{c}\right)$ and lattice distortion, as measured by resistance ratio in niobium germanium.

figure 13 shows, this can be done extensively, yielding nice little ribbons of all kinds of metal glasses, as shown in figure 14. These do have intriguing magnetic and electrical properties, vastly altered temperature coefficients, and other shifted electronics, as well as interesting mechanics. We may see much more of their application too, in the years ahead. Their processes of devitrification are also illuminating, and we may expect some overlapping of inorganic and metallic properties through the amorphous metal pathway. 


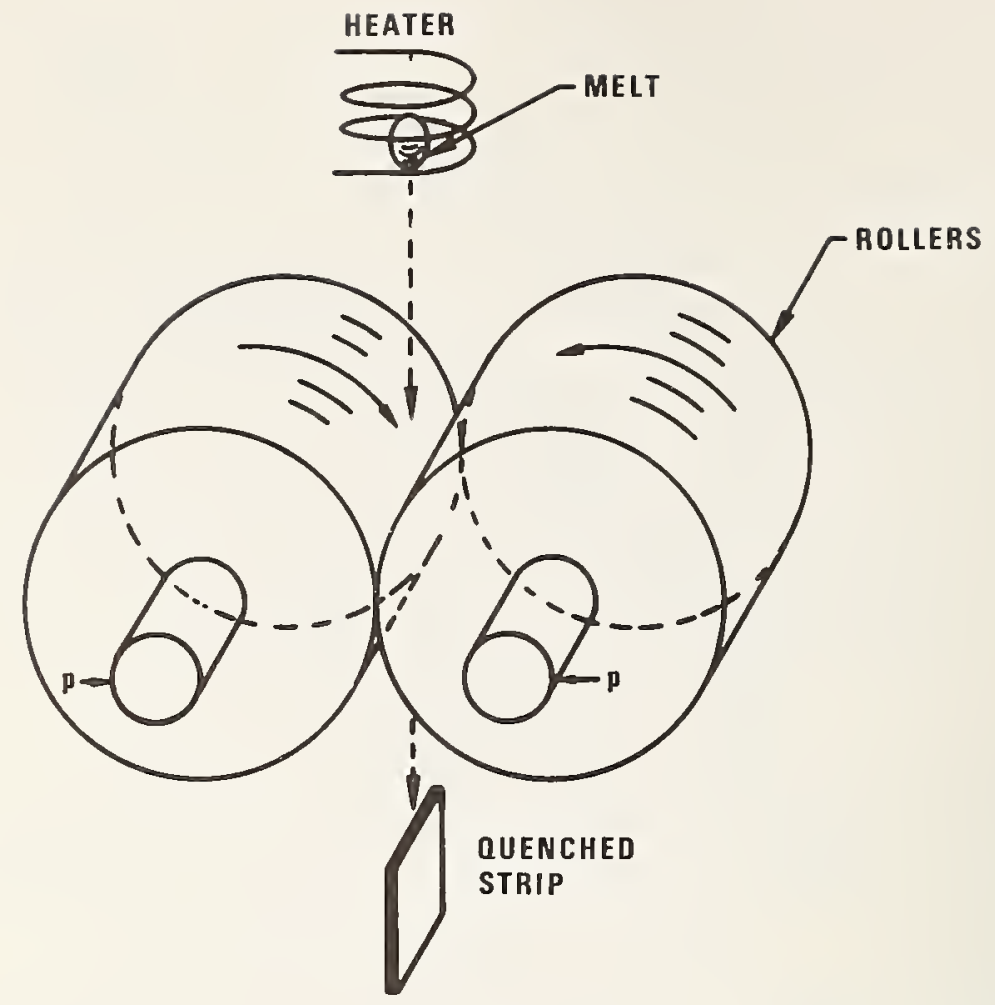

Figure 13. One type of apparatus used to quench molten alloys rapidly, producing amorphous material with unique magnetic, electrical and mechanic al properties.

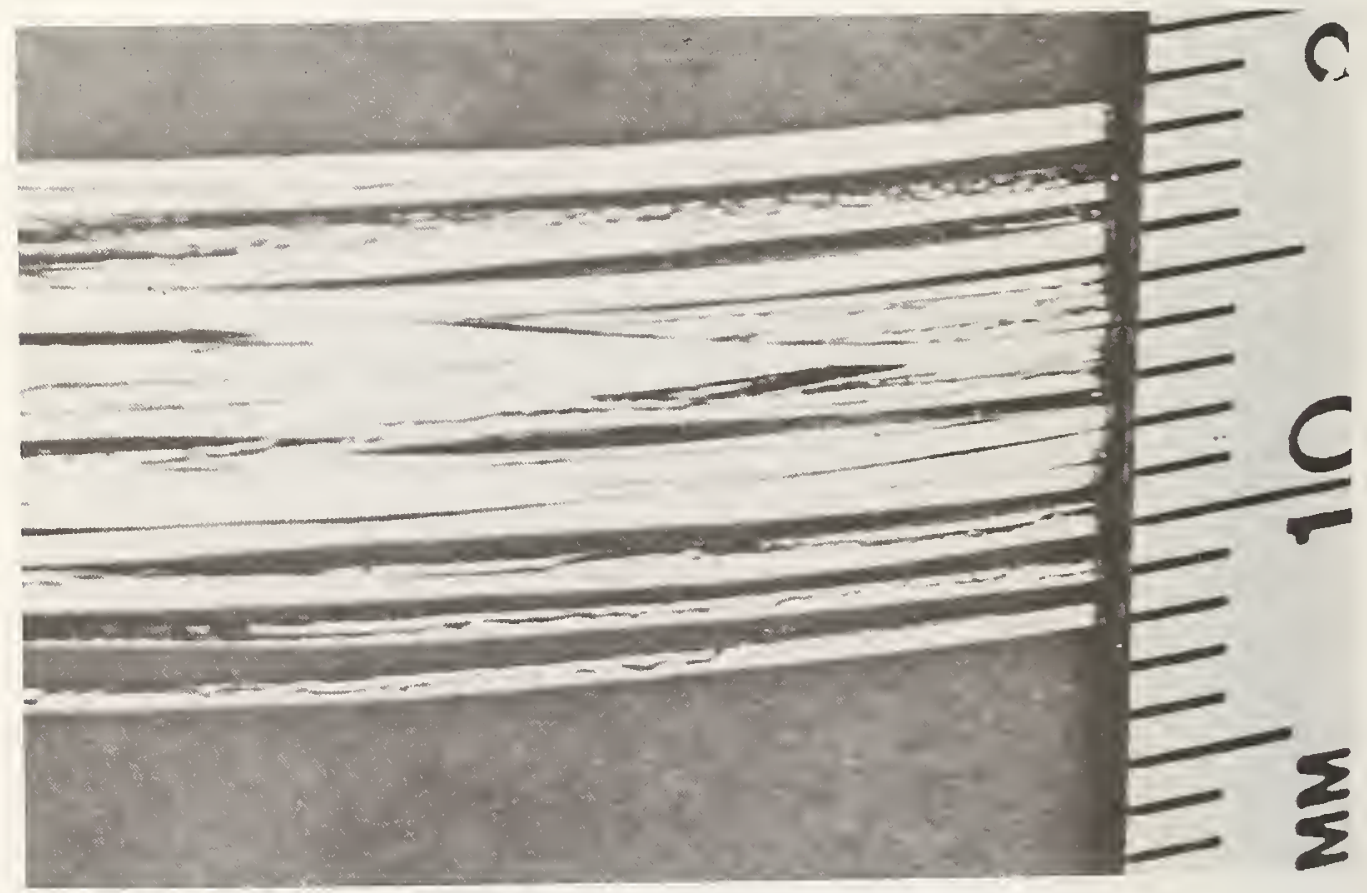

Figure 14. Glass ribbons of a cobalt-phosphorus-boron alloy produced by rapid quenching. 
But what about metallic and nonmetallic electrical properties: are they really interchangeable too? Well, the theory of Mott of years ago said that under sufficient pressure or other crystal perturbations, insulating structures and empty bands could be converted into conductors or, in other words, there should indeed be metal/insulator transitions. These too have been provided by the lattice adjustments we are accenting, as shown in figure 15 for vanadium oxide. There has, of course, been a lively polemic among theorists and experimentalists in recent years about the extension and qualifications of the Mott transition. But there is little doubt that vast new opportunities, even including new microswitches and circuit elements, exist in this kind of matter. Also various chemical and mechanical properties will surely be influenced by this versatility of electronic states.

But let us not despair that we are losing the ideal and beauty of the single crystal. In the garnets, among the most colorful of gems, we find not only the capability of incorporating appropriate magnetic ions, but also of distributing them in exquisite insulators. Here we can separate electricity and magnetism in function and, like the more complex and less adjustable ferrites, can create whole new families of insulating ferromagnets. Indeed, the magnetic domains generated here are among the most exciting sources of information processing for the future, employing, as we know, the property of digital dualism in north or south magnetic orientation.

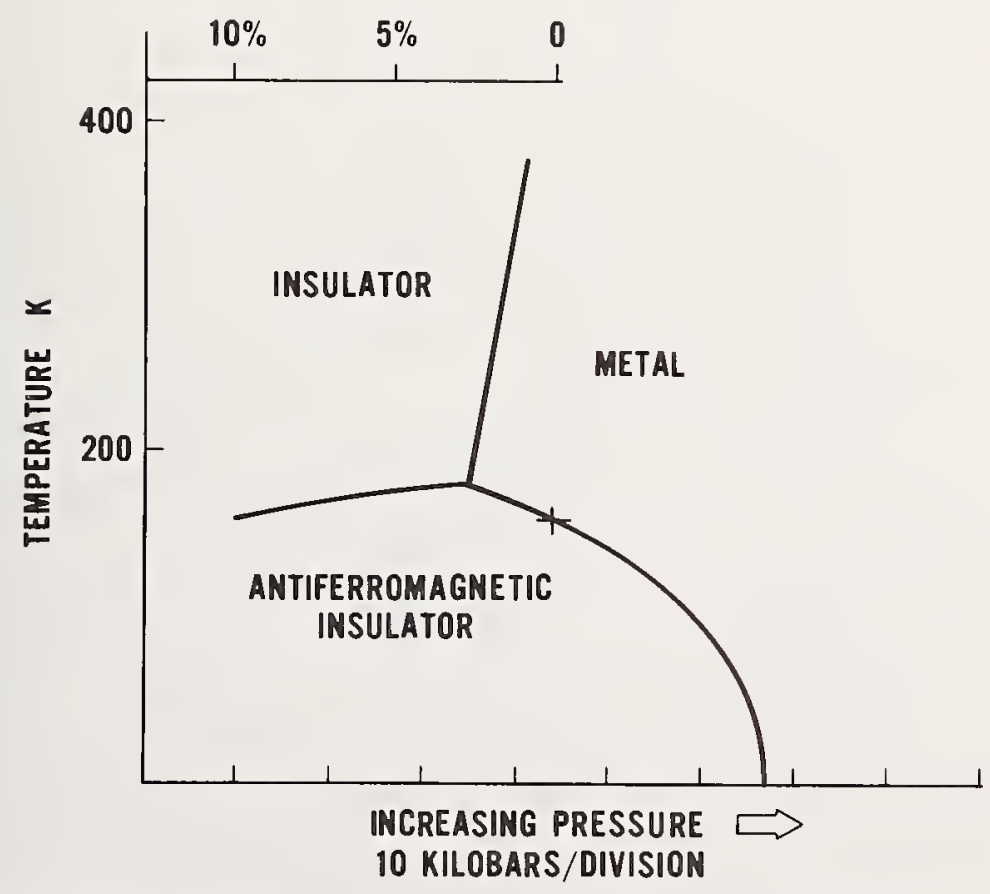

Figure 15. Metal/insulator transition in vanadium oxide with various percentages of chromium oxide. (Zero pressure point moves to left as percent $\mathrm{Cr}_{2} \mathrm{O}_{3}$ is increased, but diagram remains unchanged.) 
Figure 16 shows the rare-earth garnet crystals, with their heroically elaborated unit cells. These yield a base on which a film formed by epitaxial deposition provides an excellent source of so-called magnetic bubbles. These provide superior quality for digital memory when the composition is precisely controlled, as Van Uitert has achieved and as shown in table 2. Then, as seen in figure 17, compact thin film memories of high quality will come into increasing use to lighten and expedite all sorts of information handling. And all the attendant materials for such thin film fabrication can be controlled with similar precision.

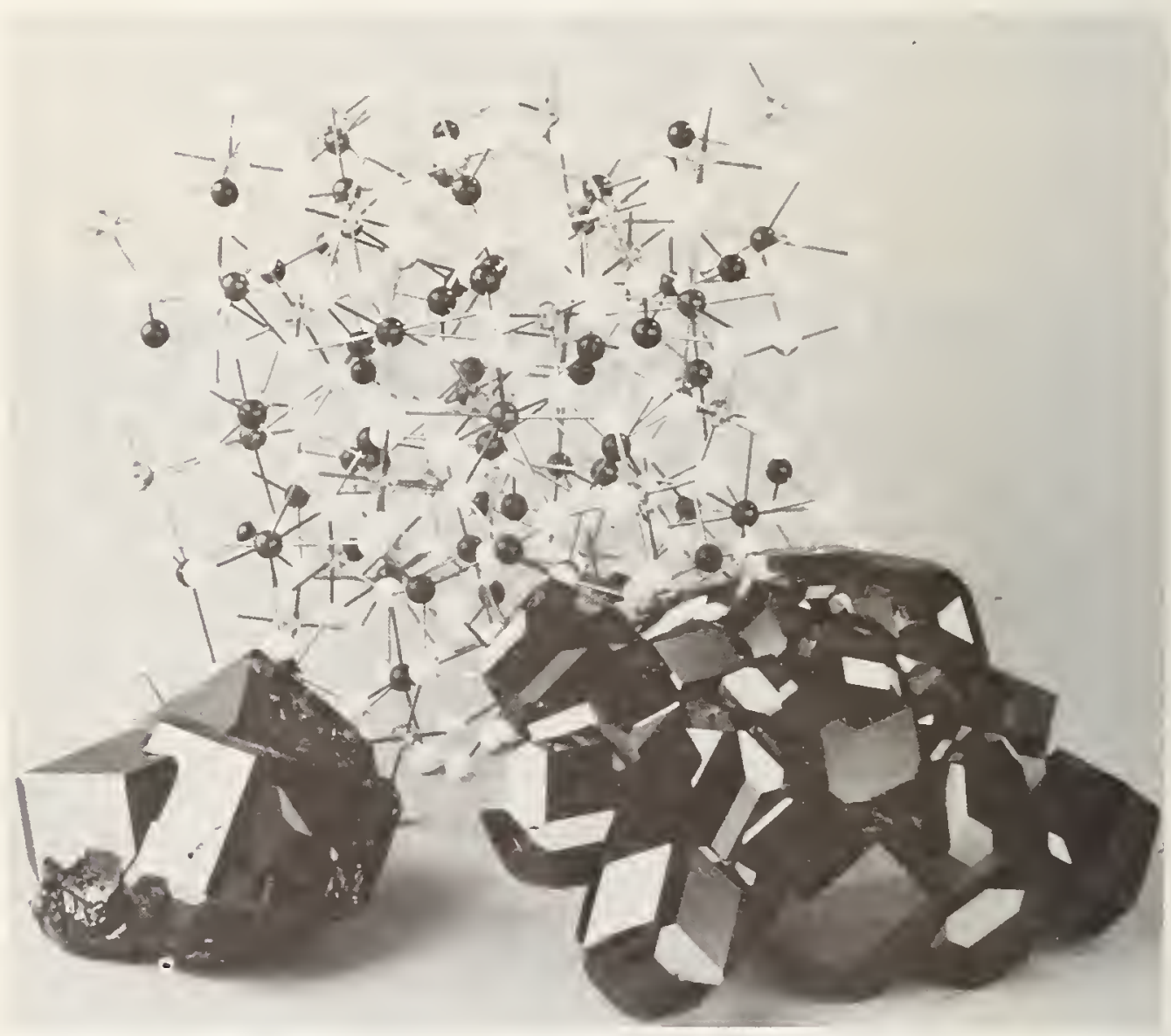

Figure 16. Garnet crystals - a source of magnetic bubbles that show promise for future information-processing devices - are metal oxides of rare-earth elements, iron and other metals. 

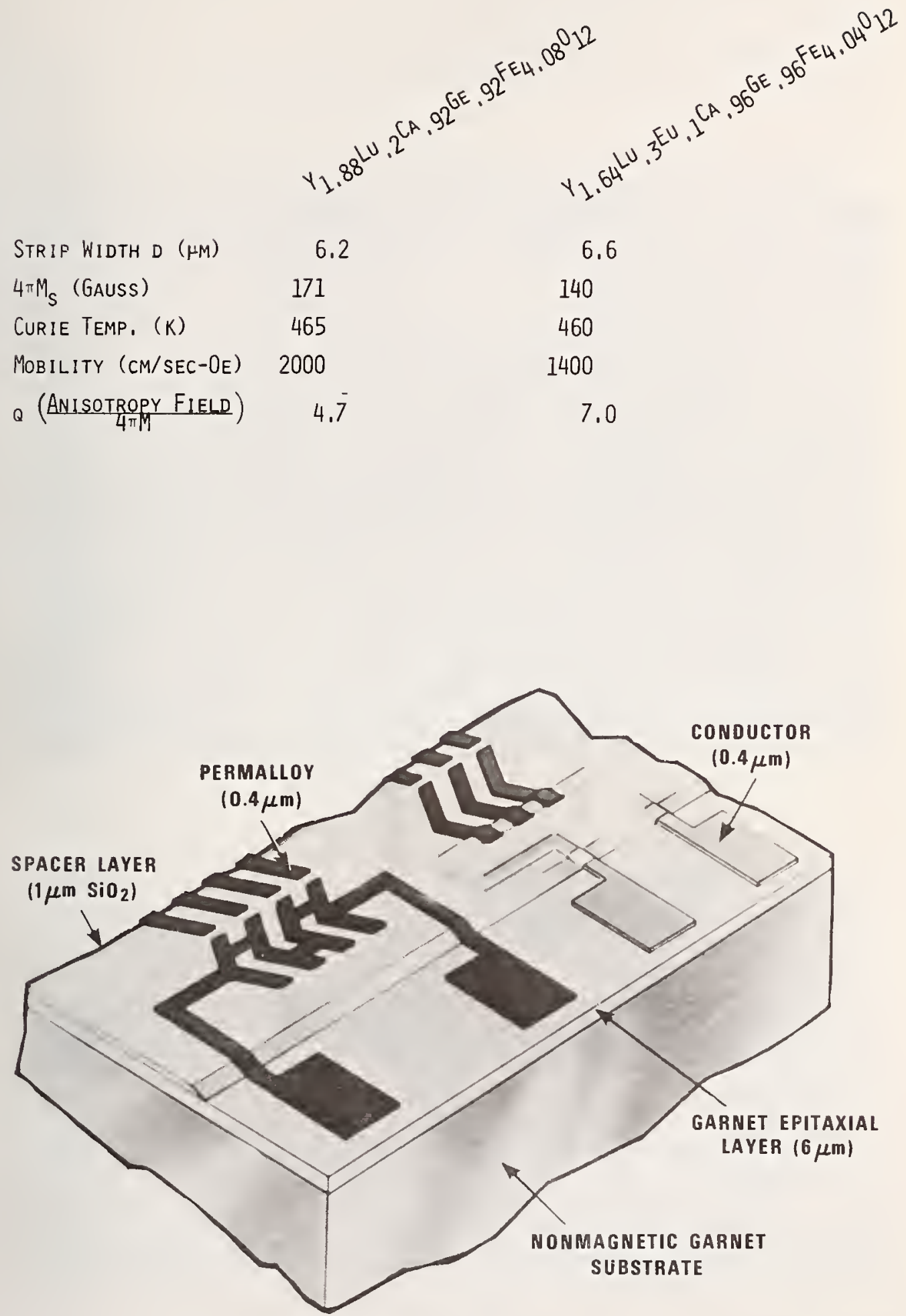

Fiqure 17. One type of magnetic-bubble memory. Magnetic layer grown epitaxially on nonmagnetic garnet substrate contains magnetic bubbles, which are attracted to permalloy structures and can be moved from one to another. 
It is the same sort of symbiotic matching of metals, maynetics, insulators and film formers that is yielding semiconductor functions through single crystal niceties as well. Thus figure 18 shows a most exciting new solid state development, the charge-coupled device for image sensing, memories and shift registers. Still other cases of materials matching occur in the now familiar but still evolving light-emitting diodes, whose uses go beyond the familiar red "dials" of watches and calculators, as indicated in figure 19.

And yet the capacities for special media built into or surrounded by crystal, whether insulator, semiconductor or metal, are even expand-

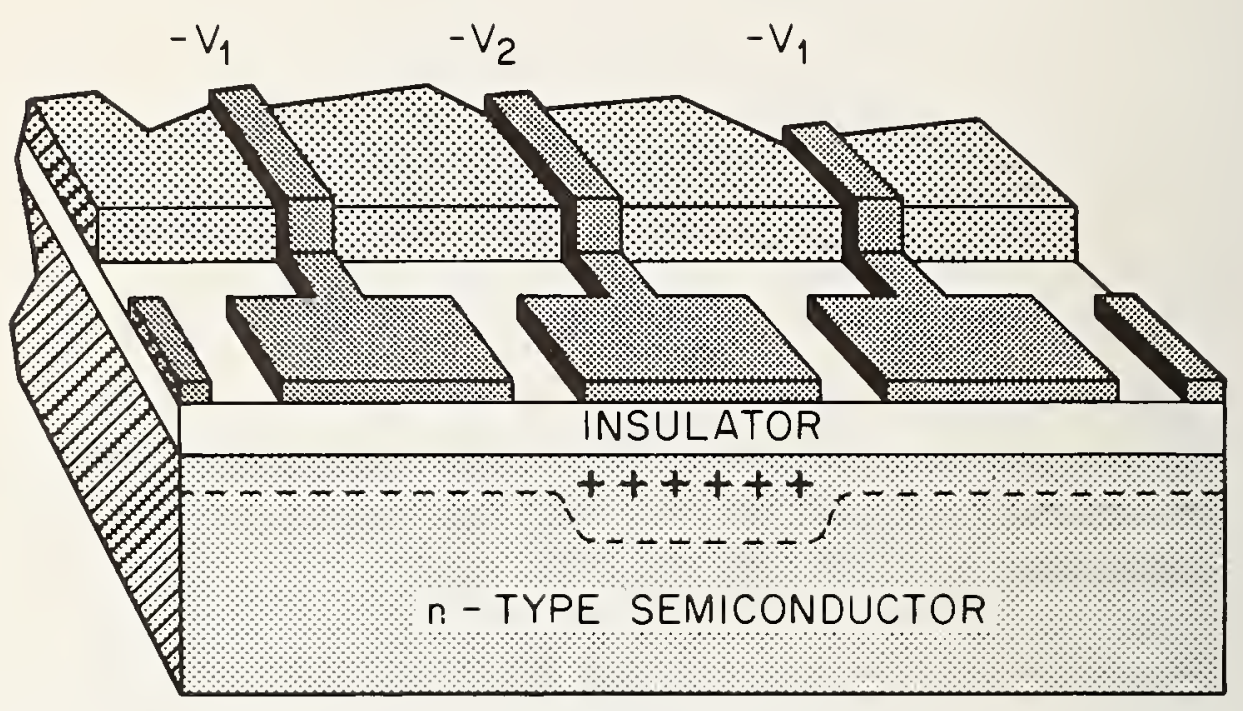

(a) STORAGE

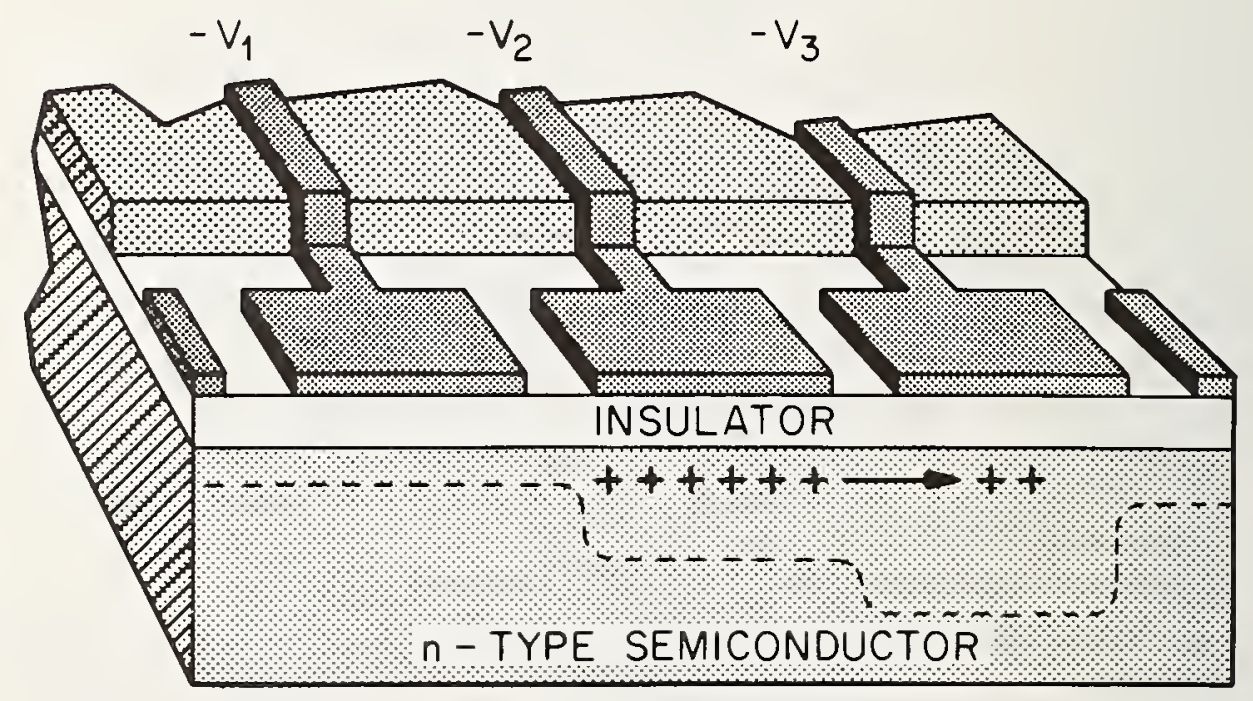

(b) TRANSFER

Figure 18. Charge-coupled devices (a) store electrical carriers in potential wells created by applying electrical potential to electrodes. The carriers can be (b) transferred from one to another by varying the potential on adjacent electrodes. 
ing. Thus figure 20 shows the new excitonic plasma switch invented recently by Auston, which depends on the metallic conduction of photogenerated, hole-electron droplets in the surface of the semiconductor crystal. This device permits digital operations about a thousand times faster than the best of the nanosecond circuits presently available and until now thought to be at the frontier of electronics.

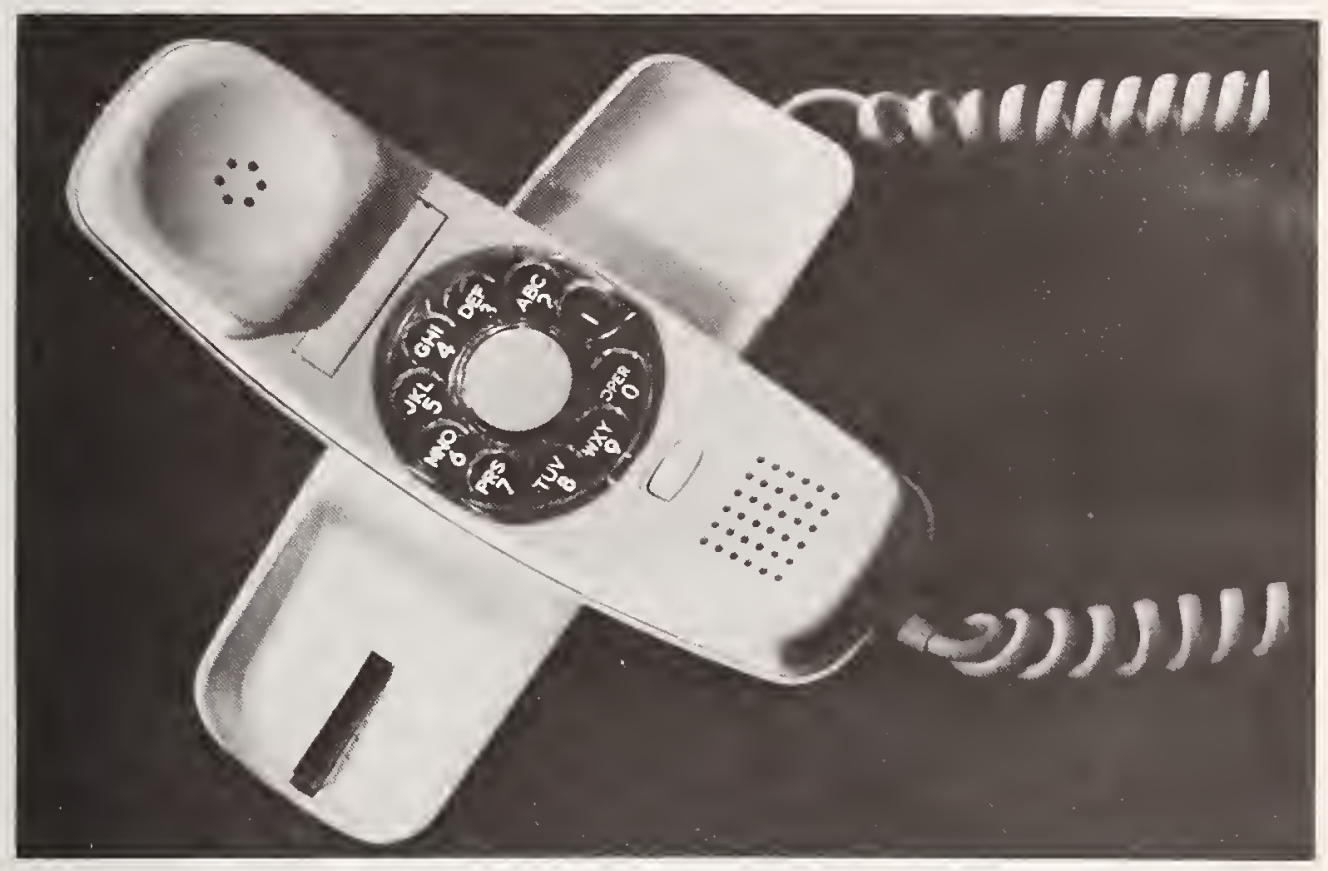

Figure 19. Light-emitting diodes illuminate the dial on the TRIMLINE (R telephone.

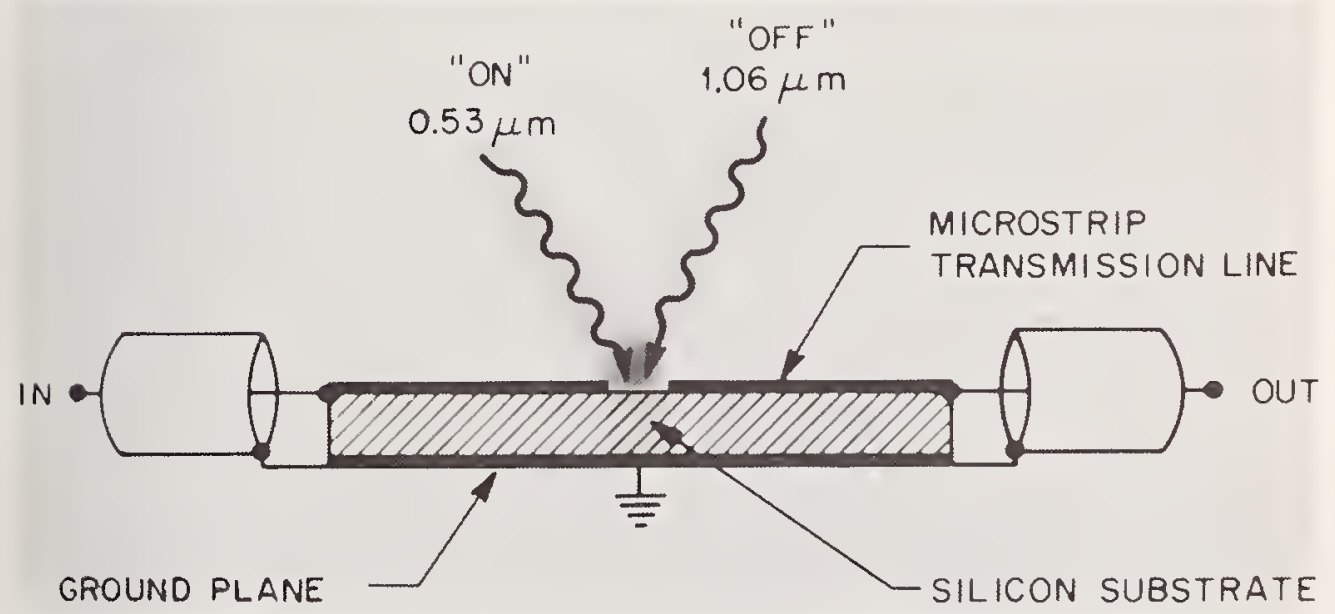

Fizure 20. New optoelectronic switch operates in only 10 picoseconds. A laser pulse focused on the gap in a microstrip transmission line increases conductivity near the surface of the silicon substrate and turns the switch "on." Another laser pulse turns the switch "off" by shorting the line. 


\section{Regularity and Imperfection in Synthetic Materials}

Up to now we have spoken of the outlook for materials science and engineering largely in terms of the natural composition of metals, alloys, gems and semiconductor crystals. We know, however, that the mid-century was characterized particularly by an equally dramatic advance in synthetic substances for structural and mechanical as well as electrical functions. Preeminent among these are the high polymers, bases for rubber, plastics and fibers, which have, in their turn, been as central to social and economic progress as the inorganic and metallic structures. Thus figure 21 represents in one industry alone, one that for the past quarter-century has reflected about 8 or 9 percent of the national annual capital investment, the growing role of synthetic substances in making the actual plant, from which our goods and services are derived. Similar representations would exist for the textile industry, whose consumption of polyamides and polyesters now far exceeds, and ecologically transcends, its classic dependence on cotton, wool and silk.

The polymers listed in figure 21 , as well as those used in the fiber industry, and a huge volume of plastics (but not all) contain a large fraction of

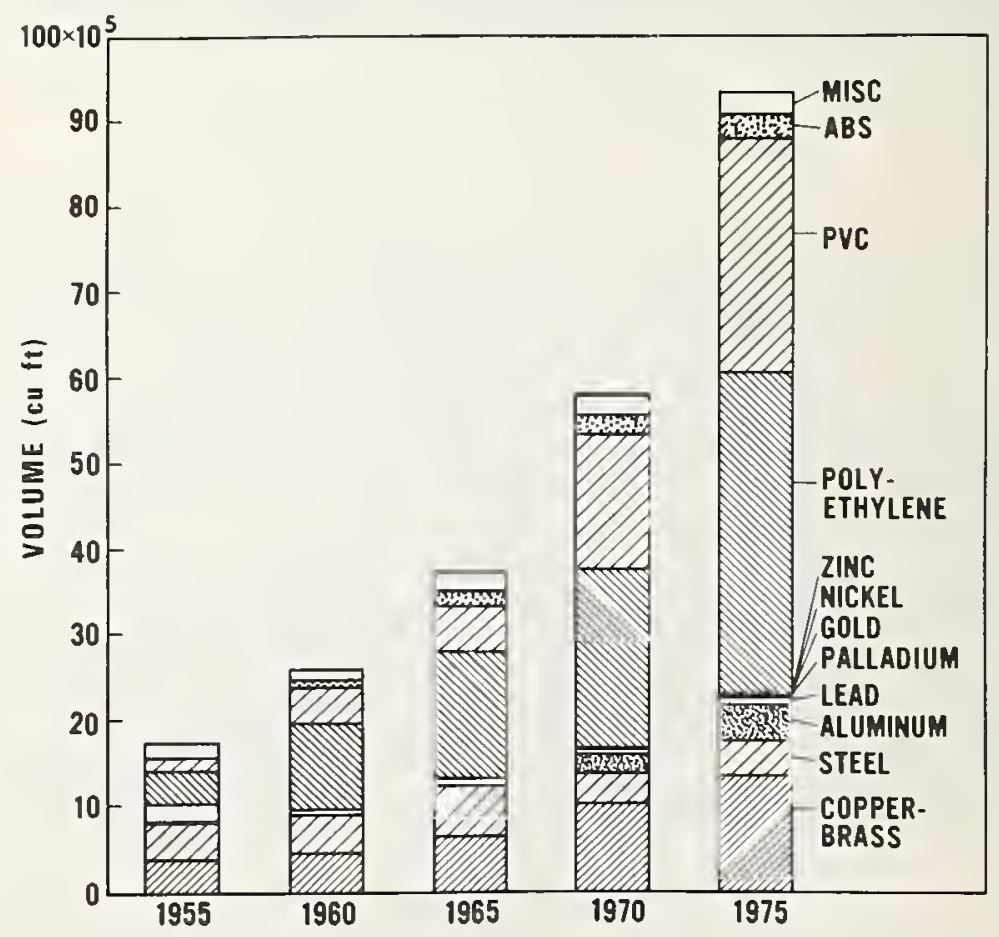

Figure 21. Bell System consumption of plastics and metals over the last 20 years shows growing role of synthetic substances. 
crystallinity like the inorganic matter we have reviewed. This microcrystalline state is shown by the electron micrograph in figure 22 , where there is a connective matrix of disordered matter whose qualities and nature we have sought to specify since 1940. In these last three decades, controlling both crystallinity and the packing of chain molecules comprising most of these synthetic systems have been memorable parts of the role of polymer science and technology in the world economy and service. Thus figure 22 but symbolizes an elegant and complex morphology, in which sheaves of crystallites of ultramicroscopic dimension, often aggregating in spherulites, are themselves connected by chain filaments into a remarkable medium having both liquid and crystalline characteristics. Again idealism is unlikely, even in the ultimate chain structure, although some condensation polyesters and polyamides come very close to it.

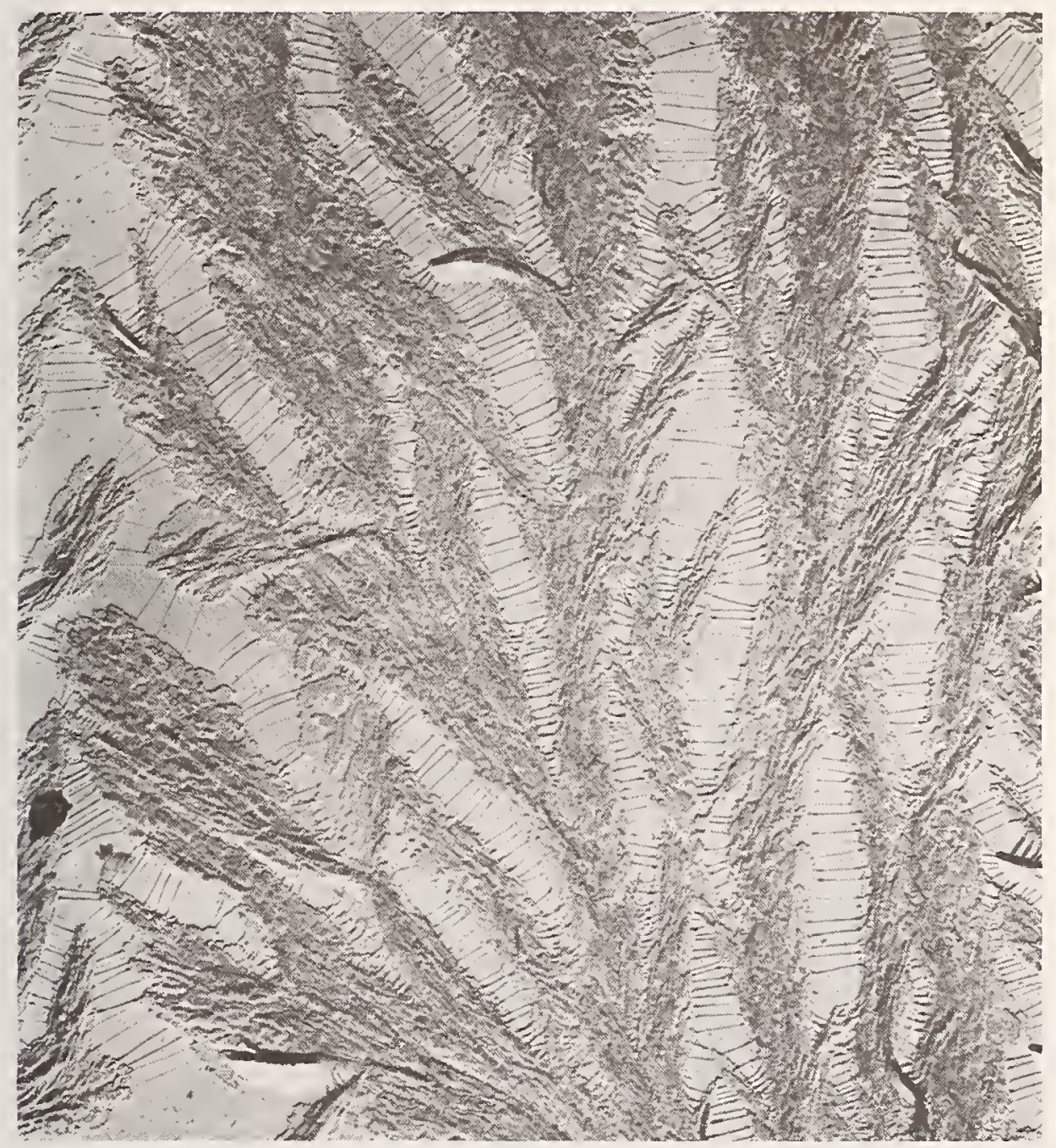

Figure 22. Crystallinity of polymers: electron micrograph $(30,000$ magnification $)$ shows tiemolecules joining typical sheaf-like aggregates of dense polycrystals. 
The nonideal is common, however, as in the most important single class of materials in the field, polyolefins, particularly polyethylene. Polyolefins contain branches, which brings us back to the theme of learning to deal with real solids and matter in the future, to recognize and take advantage of the versatility of the nonideal. Thus figure 23 shows the current characteriztion of branches of the real carbon chains in polyethylene, as determined by careful microscopic and geometric studies of (thankfully) relatively standard materials provided by the NBS.

These branches bring us at once to another quality of matter, which we have emphasized little so far: chemical stability or reactivity. This property, as we did in fact remark, is really dominant in the corrosion of metals and other conversions by imperfections and impurities in the crystals. Here in polymers a similar feature obtains, with the chemical realities being much affected by both impurities and chain abnormalities.

\section{NBS BRANCHED POLYETHYLENE FRACTION 5AS5}

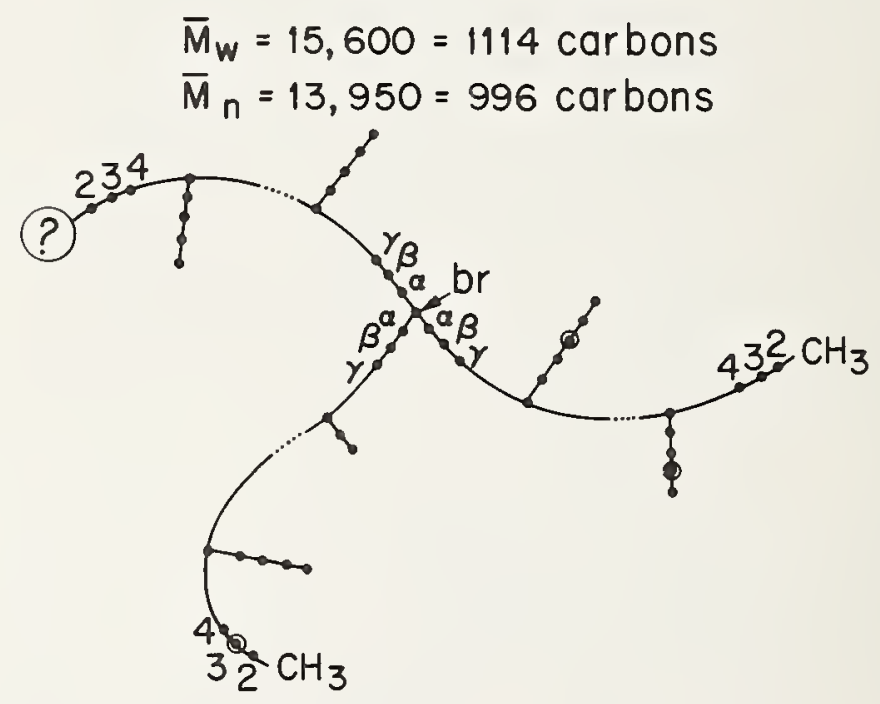

\begin{tabular}{cccc} 
Branch points & per wt. av. mol. & per $1000 \mathrm{CH}_{2}$ \\
\hline Ethyl $\left(\mathrm{CH}_{3}\right)$ & $:$ & 1.3 & 1.2 \\
Butyl $\left(\mathrm{C}_{2}\right)$ & $\vdots$ & 5.8 & 5.2 \\
Amyl $\left(\mathrm{C}_{3}\right)$ & $:$ & 1.7 & 1.5 \\
Long $\left(\mathrm{C}_{3}\right)$ & $:$ & $\frac{1.0}{9.8}$ & $\frac{0.9}{8.8}$
\end{tabular}

Figure 23. Characterization of branching in NBS branched polyethylene fraction 5AS5, determined by microscopic and geometric studies. 
Likewise stabilization - a vital quality of these systems, enabling their real use, their technical and industrial service - is affected by the distribution of stabilizers in the solid, as governed by the crystallinity and in-phase relationships. Thus in figure 24 is seen the crucial quality of polyethylene, or indeed other polyolefins, as engineering and commercial materials. This quality is resistance to oxidation in the atmosphere, to which they are fundamentally vulnerable. However, as shown in the curve and reflected particularly in the work of Dr. L. Hawkins at Bell Laboratories, now applied throughout the world, it is possible to prevent oxygen uptake and thus degradation of polyethylene for such periods as to make it a highly effective industrial substance. Properly compounded, polyethylene sustains a 40 year life, already demonstrated in cable sheath and in many other demanding mechanical and electrical applications.

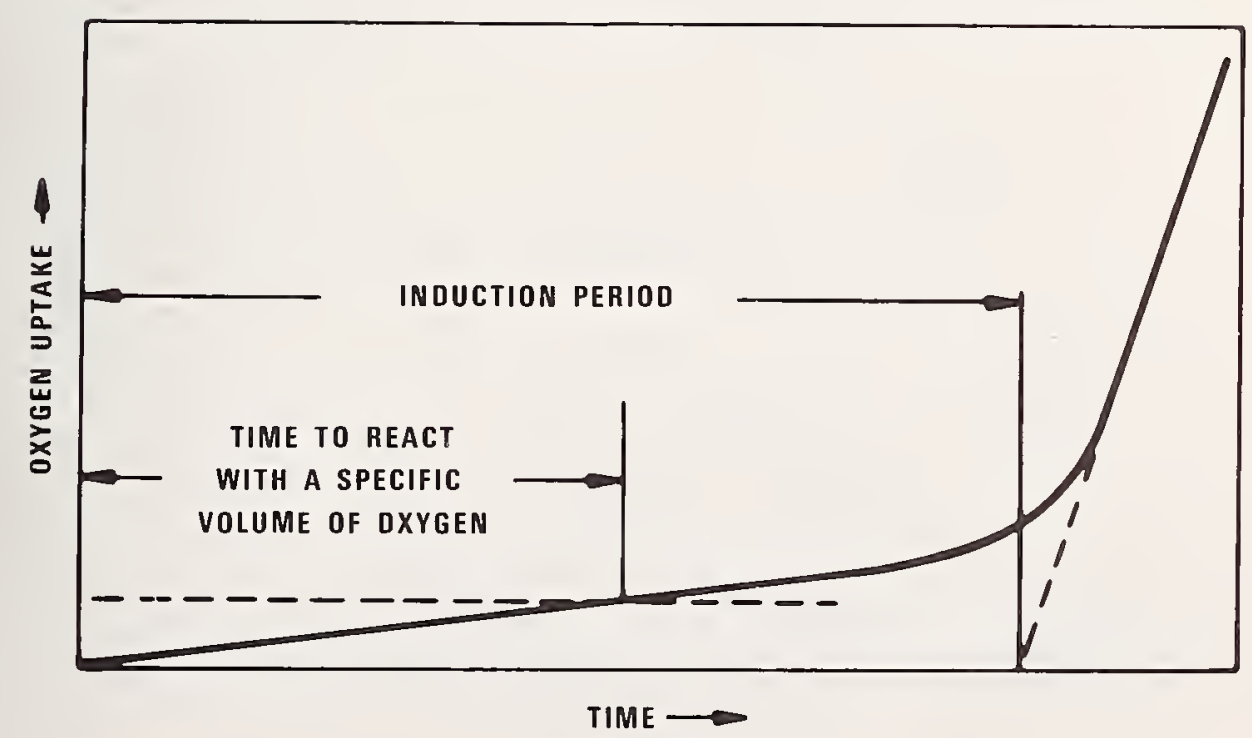

Figure 24. Schematic of oxidation of polyethylene, with stabilizers controlling its degradation. 
So, we can control and understand the reaction mechanisms summarized in figure 25 well enough to address the next issue in the future of synthetic polymeric matter. That is, to what extent can we expect important properties of polyethylene to conform to the ideal chemical composition of its inert hydrocarbon? Figure 26 indicates that the approximation with respect to moisture absorption, a vital matter in packaging, in electrical properties, in the use of barrier and protective material, is pretty good. Nevertheless, it is not ideal, and we must deal with it accordingly. Years of exposure of polyethylene to the high-pressure hydrolysis of the sea bottom indicates that the effects are indeed as modest as shown. Figure 27 illus. trates a similar reality for the processing necessary for such high polymers: namely, the hydrocarbon chains do acquire some polarity and undergo some alteration in composition, bois of which are altogether manageable.

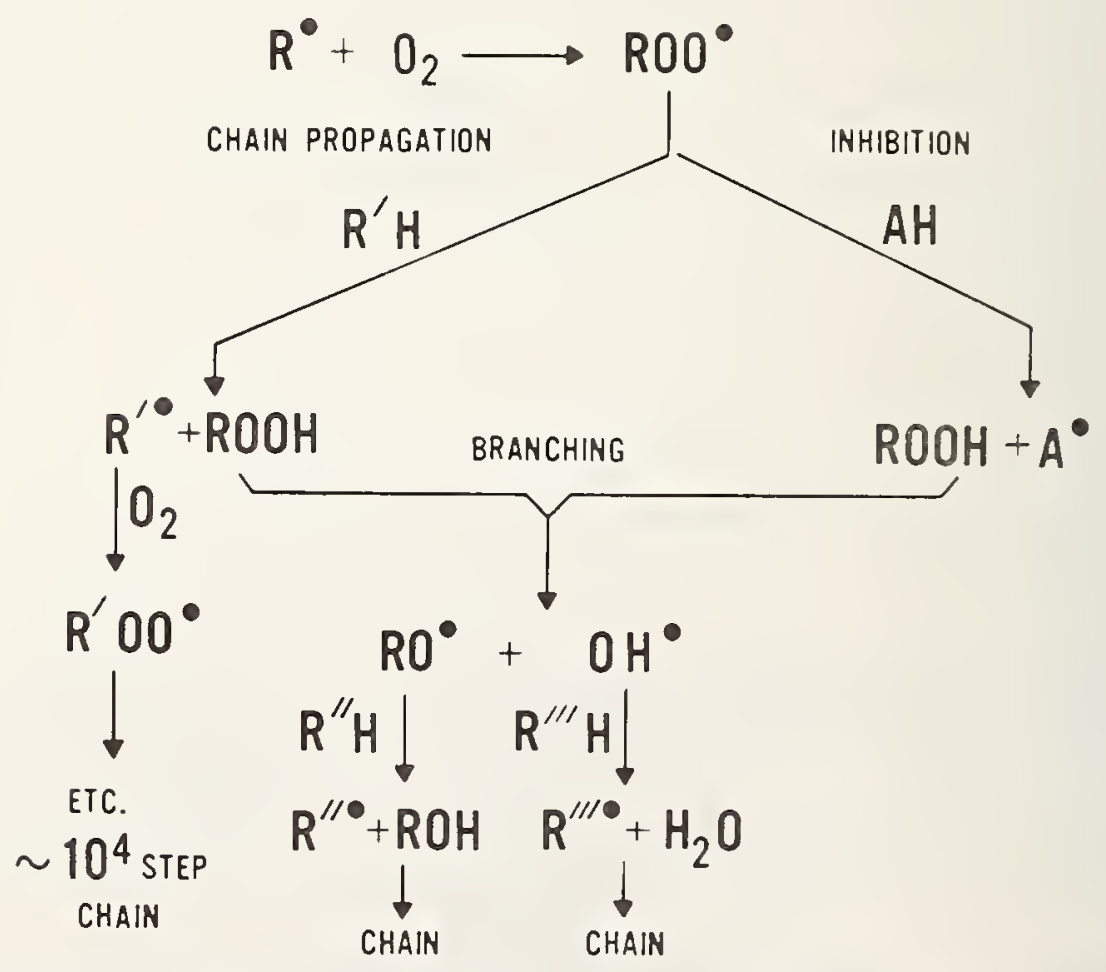

Figure 25. Mechanisms of chain propagation, branching and inhibition in polymers. 


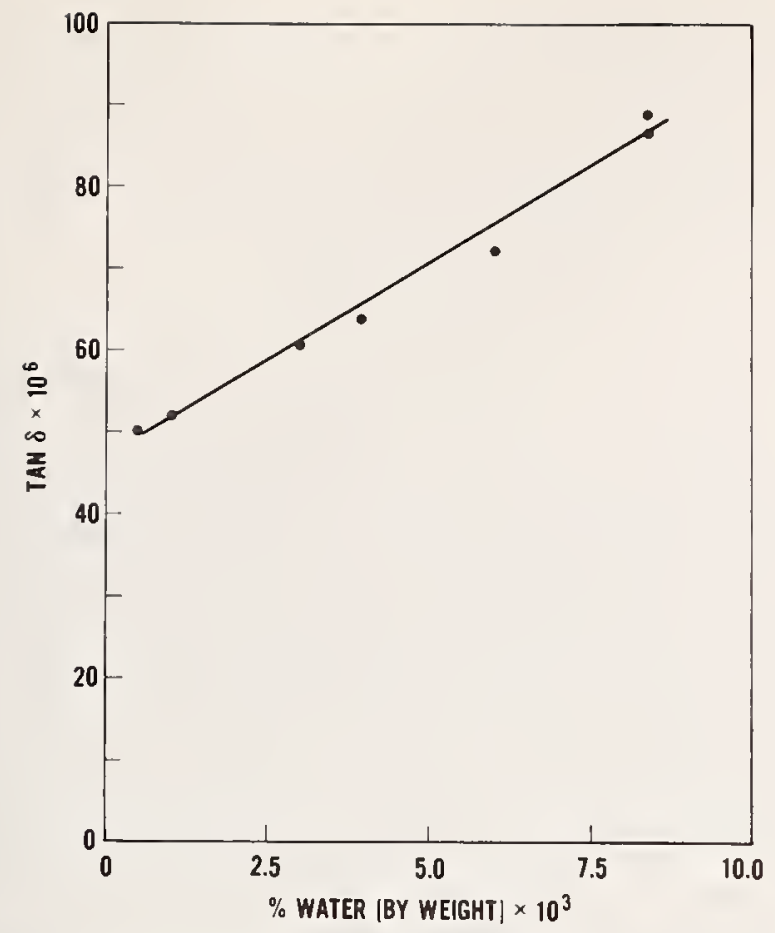

Figure 26. Dielectric properties of ocean cable (SG) polyethylene at $30 \mathrm{MHz}$ as a function of moisture absorption.

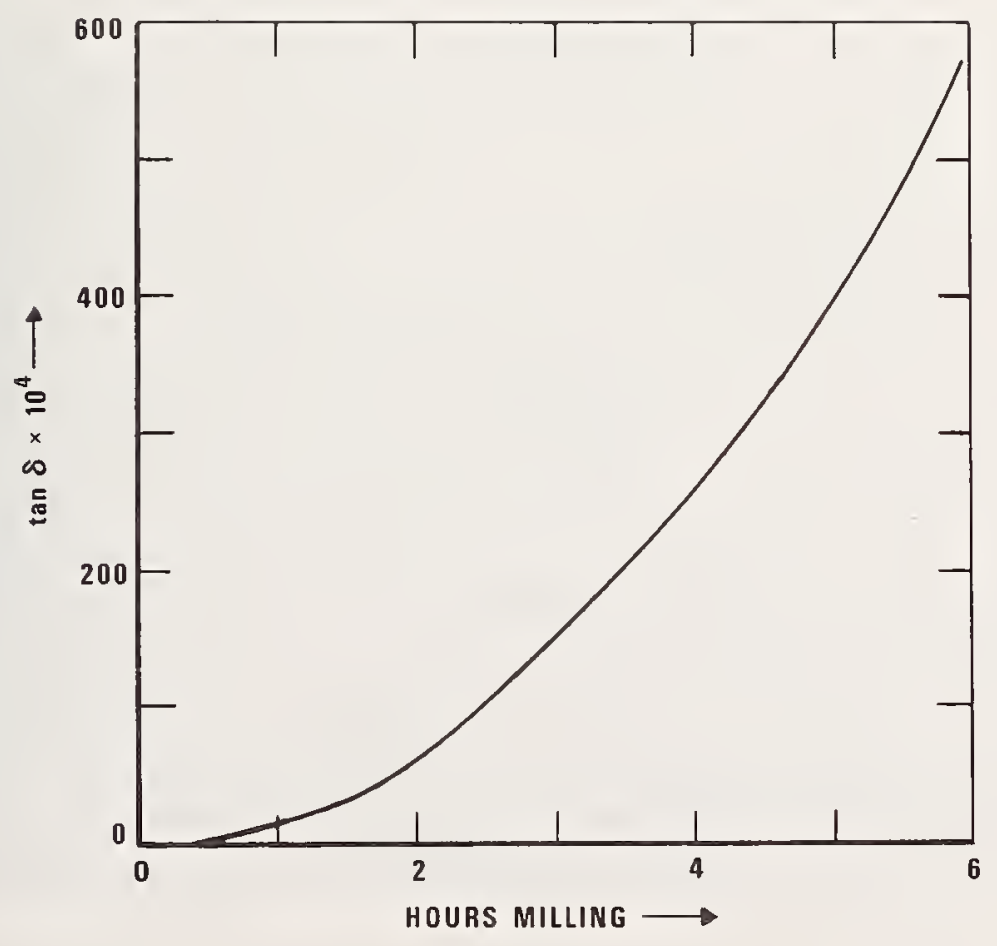

Figure 27. Effect of processing on the dielectric properties of polyethylene. 
Let us shift now to consideration of whether these synthetic polymer structures, having the degrees of idealization through synthesis that we have discussed in the past few examples, can also be adapted to particular mechanical and electrical functions. These functions are often dominated by designs and operations, but they frequently have little to do with the nature of the material or the economy of processing.

Figure 28 illustrates such a case-the wires used in communications and other electrical distribution systems. These have long been insulated and protected by composite sheaths of textiles, lacquers and other ingredients. The question is whether molecular design and synthesis can substitute for these traditional and time-proven assemblies. On the one hand, it is desirable to have rapid extrusion by the use of thermoplastic economy at many-miles-a-minute fabrication speeds. On the other hand, it is desired to have stable nonplasticity but high elongation and flexibility in use. So, processing of polymer chains as thermoplastic is desired, with subsequent cross-linkage, as is done in vulcanization but at many times the speed of such reactions. Similar reactions are also used in making all sorts of thermoset and casting materials. As shown in figure 29, this alteration of structure can be quickly achieved by activating copolymerized double bonds with electrons from a small accelerator attached to the production

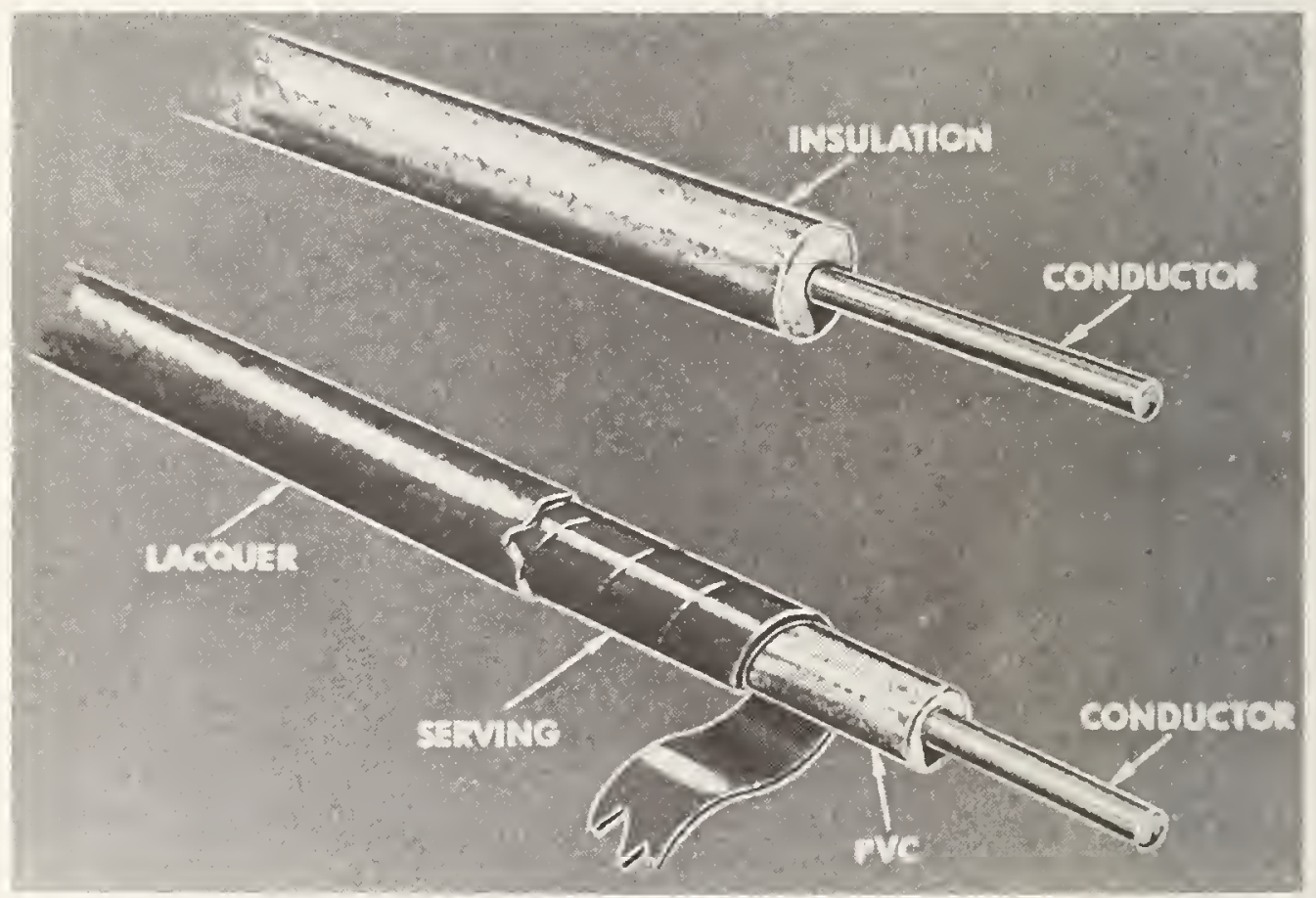

Figure 28. Electrical distribution wires. (Top) new cross-linked polymer coating and (bottom) previous textile-served desionn. 


\section{(A) AS EXTRUDED}
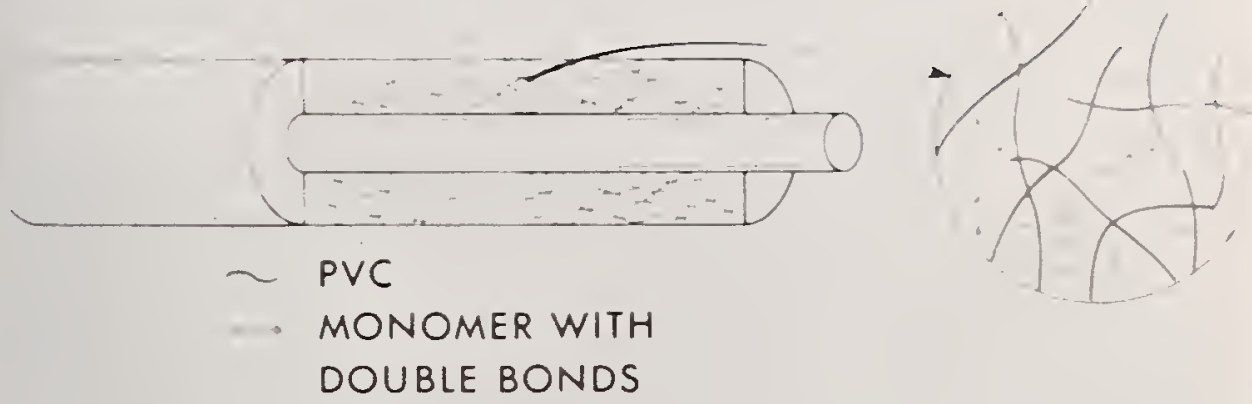

(B) AFTER IRRADIATION

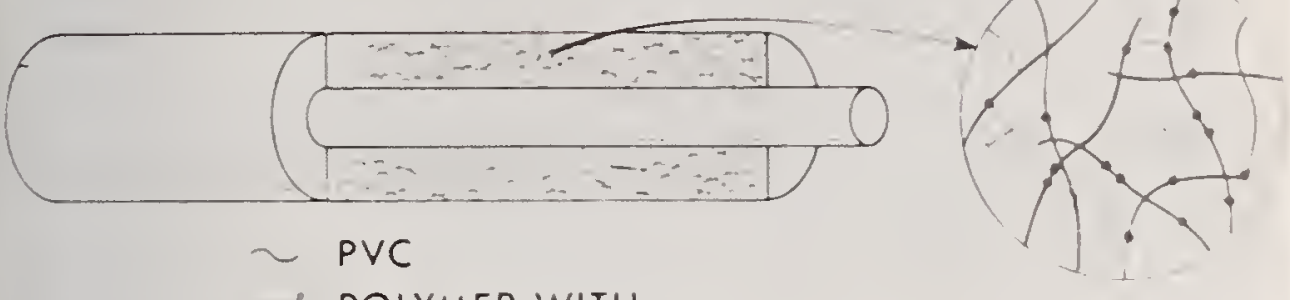

\section{POLYMER WITH \\ PENDANT DOUBLE BONDS}

Figure 29. Irradiation activates copolymerized double bonds to form cross-linked polymer for wire coating. Molecules of polymerizable monomer (tetraethylene glycol dimethacrylate) at tach to polyvinyl chloride and to one another, producing a net work in single extrusion over wire. 
line, as seen in figure 30 . In this way, the management of complex arrays and assemblies of wires requiring frequent readjustment or interconnection can be vastly improved, replacing such aggregations as shown in figure 31.

Such modifications of material structure are increasingly widespread in many other uses, such as the preparation of plastic ducts, tubes, plumbing fixtures and equipment for the chemical process industry. Still other modifications of solids are under development, such as the use of expanding agents to form homogeneous bubbles, making expanded dielectrics and structural insulating and thermal barriers available. An example of the economy and efficiency involved is shown in figure 32 , where high-density polyethylene has undergone a 50 percent expansion in volume on extrusion, using the techniques of Dr. R. Hansen.

In addition to these adjustments during polymer processing, other major pathways to new materials and designs are offered by extensive chemical modification of polymer structure before processing. Most excit ing is the use of composites, of which polymer carbon fibers, mistakenly called graphite, are the central elements. Here it was found more than 20 years ago that various chain and net structures, after suitable chemical treatment and pyrolysis, would yield increasingly dehydrogenated network solids.

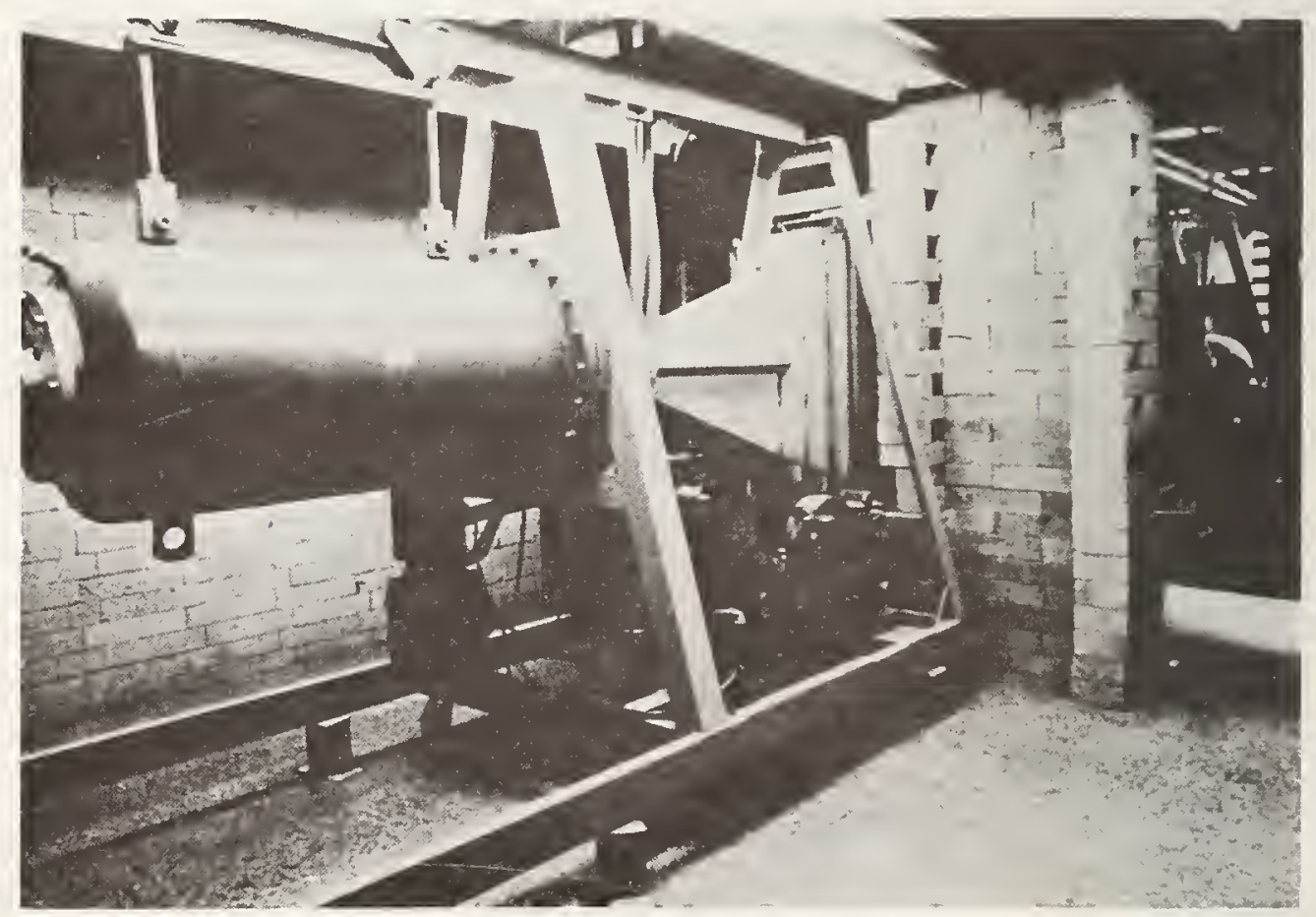

Figure 30. Western Electric electron accelerator used to produce cross-linked polymer wire coating. 


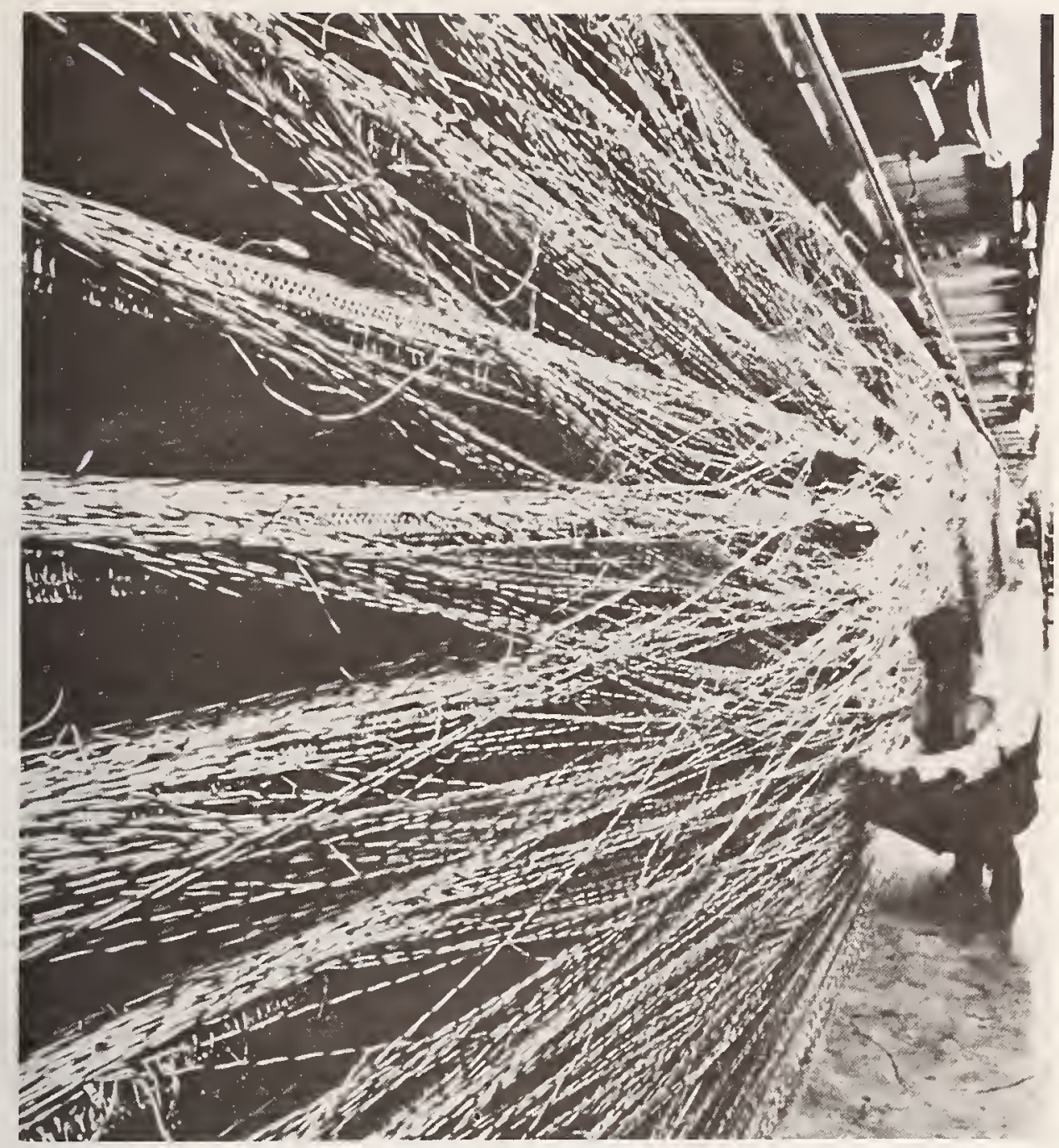

Figure 31. Main distributing frame in telephone central office, where complex wiring con. nects individual telephone users to the nation wide net work.

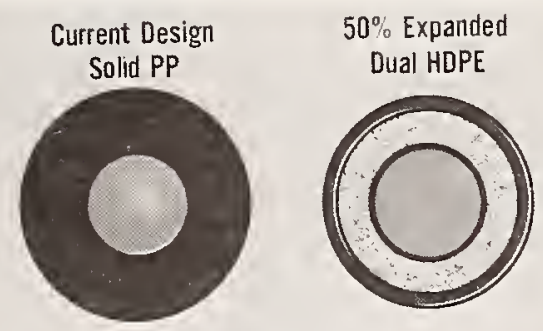

Relative Cable Cost

Copper 1.00

0.93

Aluminum 0.87

0.71

Figure 32. Waterproof cable insulation: design and relative cost of current solid polypropylene and new dual-expanded high-density polyethylene consisting of a foamed plastic core and thin solid plastic skin. 
One such case is polyvinylidene chloride, illustrated in figure 33. Figure 34 symbolizes a related transformation using polyacrylonitrile. Here again, highly conjugated ring structures are created, and eventually polymer carbon networks of extreme hardness and stability are attained. This process is particularly dramatic in the case of previously cross-linked
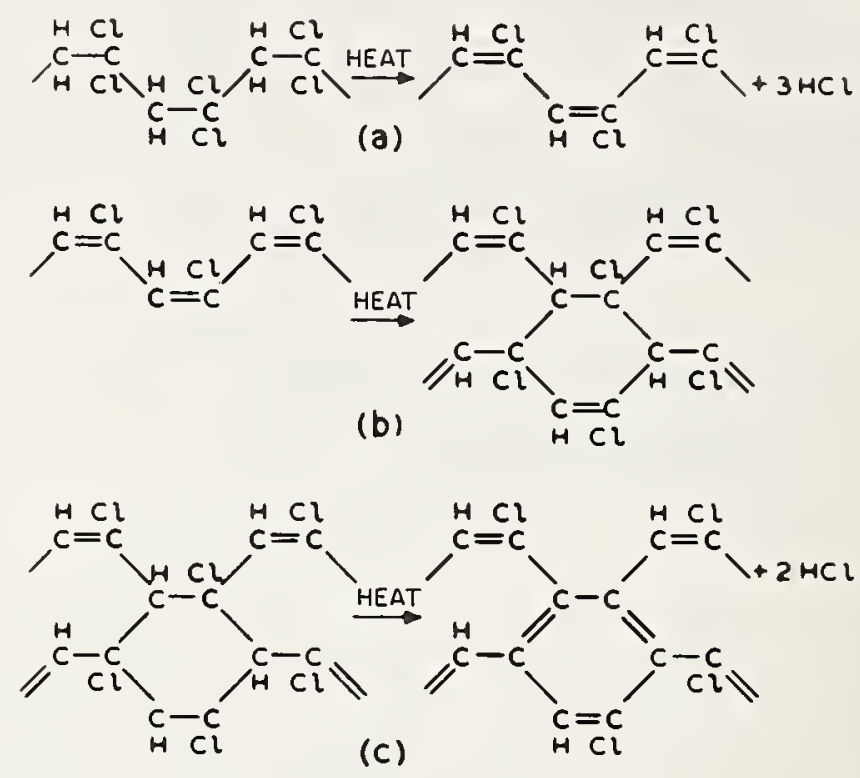

Figure 33. Conversion of polyvinylidene chloride to condensed ring polymers.
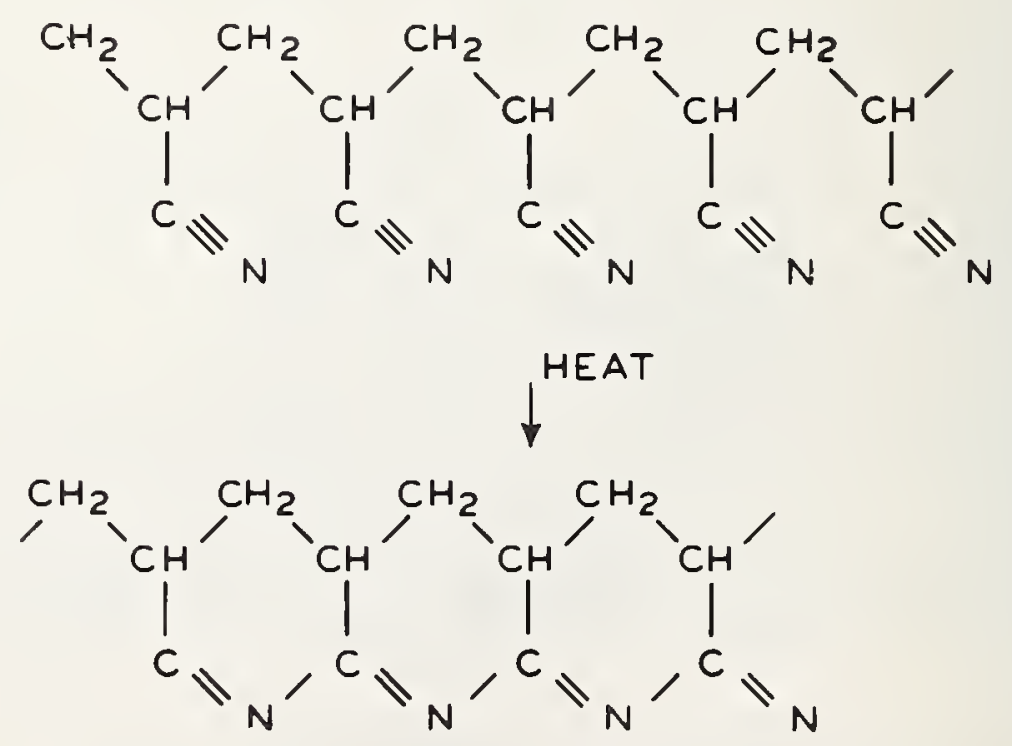

Figure 34. Conversion of polyacrylonitrile by heat; further conversion is almost certain. 
ring polymers, such as polydivinyl-benzene and its copolymers. Here, with appropriate preoxygenation. exceedingly rigid networks of vitreous carbon are obtained, as seen in figure 35 . The whole solid structure is dominated by the resonance bonding of the $\pi$ electrons, which spread throughout the macroscopic sample after initial excitation. This is demonstrated during

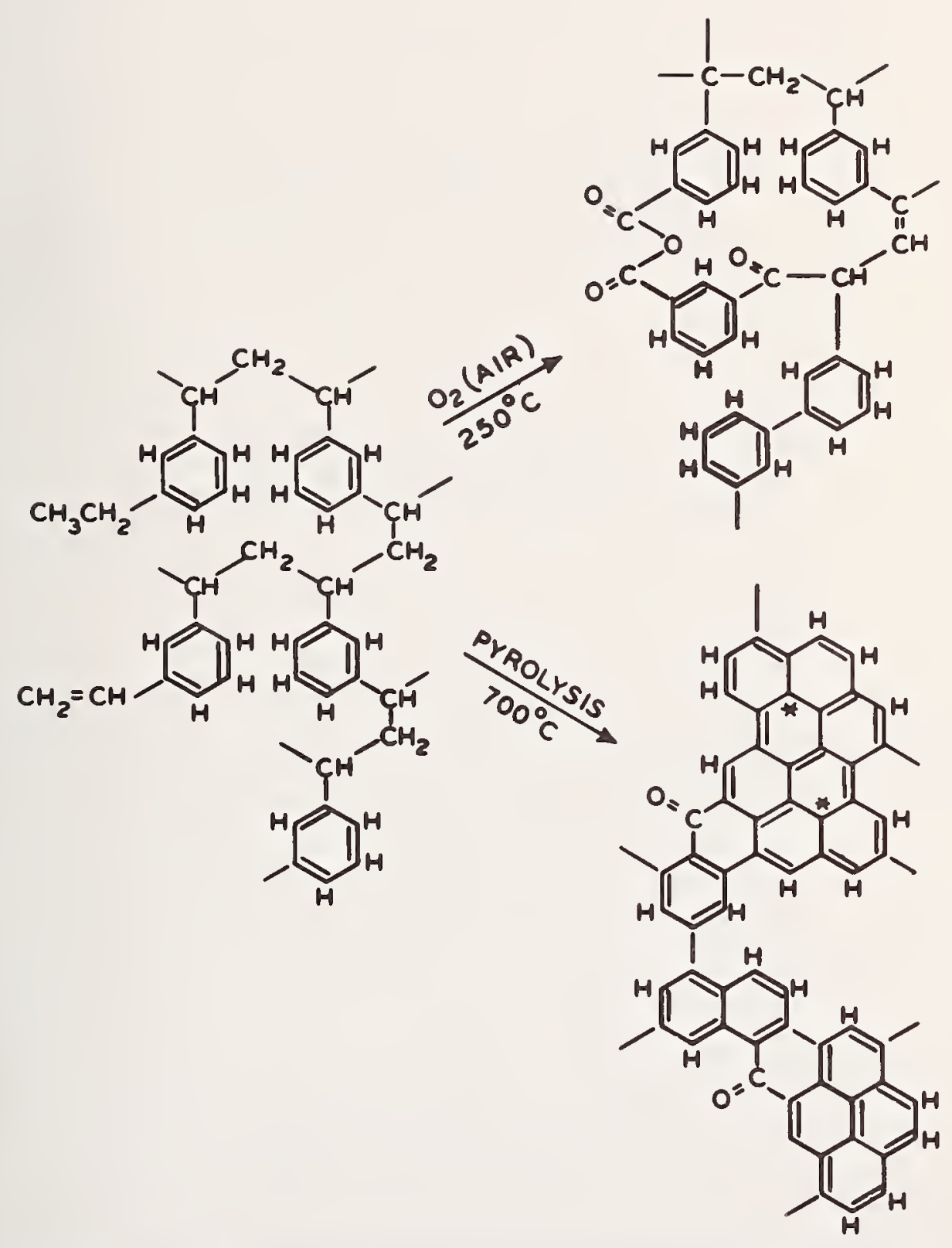

Figure 35. Schematic conversion of divinylbenzene/ethylvinylbenzene copolymer to electronically active derivatives. 
the formation of the polymer carbon solid by a typical maximum in the paramagnetism, illustrated in figure 36. The dehydrogenated side of this maximum is, of course, characterized by relatively high electrical conductivity, as the resonating electrons are shared throughout the whole solid and confer extraordinary stability mechanically and chemically on the cross-linked carbon rings, as shown in figure 37.

Indeed, it is this effect that was first exploited in the formation of ablative heat shields for rockets and space vehicles, whose return to earth is

\section{TEMPERATURE OF PYROLYSIS IN DEGREES CENTIGRADE}

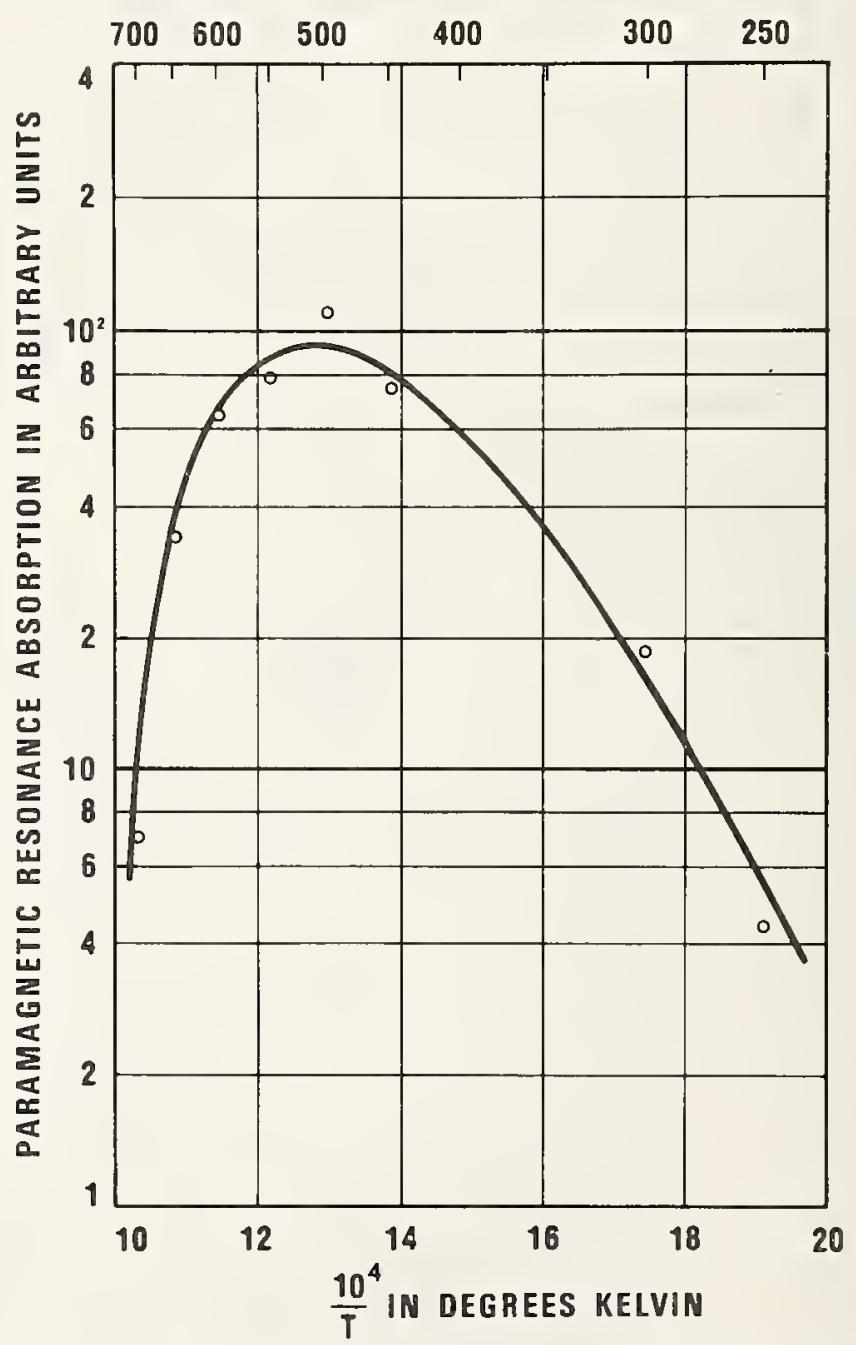

Figure 36. Paramagnetic resonance absorption in preoxidized polyvinylbenzene as a function of progressively higher temperatures of pyrolysis. Typical maximum in paramagnetism demonstrates resonance activity of $\pi$ electrons. 


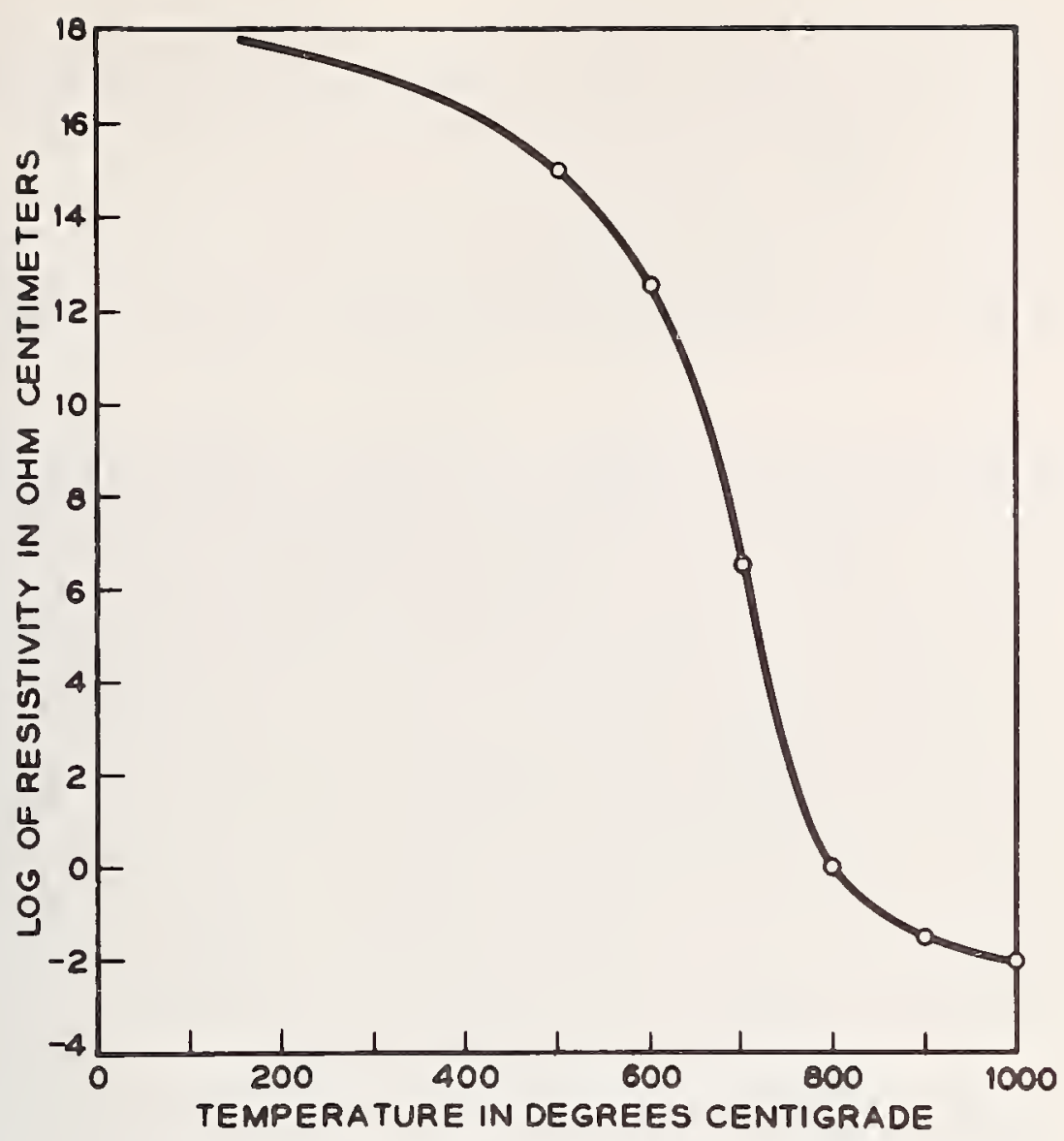

Figure 37. Specific resistivity of preoxidized polyvinylbenzene pyrolysates. Sharing of electrons produces characteristically high electrical conductivity.

protected in the atmosphere by the actual in situ reaction converting the polymer to a rigid, stable and refractory shield that withstands at least $7000^{\circ} \mathrm{F}$, as indicated in figure 38 . Precision of shape retention, even with the volume shrinkage necessary in this reaction, is significant in recalling the fiber formation that is typically employed in the creation of composites.

The history of these composites is relatively familiar through the ambitious effort to use them in the RB211 Rolls Royce jet engine big fan. While, for many reasons, that program was abandoned, the casting (epoxy) polymer/polymer carbon fiber composites have, since 1971, played an increasingly important role in high-modulus, highly elastic and lightweight structures. In the field of sporting equipment, tennis rackets, fishing rods and golf-club shafts made of these systems have outperformed any others in history. Presently taking shape is an important application of such systems, proposed when they were first discovered in the 1950's: their use in demanding functions in equipment for chemical and process industries. The durability and chemical inertness of polymer carbon composites outperform any known economical metal or ceramic structures. The Babcock 
and Wilcox Company is now making large experimental ducts and valves for process applications, which appear to offer great advantages. We shall expect to see critical parts of chemical plants and energy-conversion systems made of these composites in the relatively near future.

An interesting insight, supporting $\mathrm{x}$-ray scattering and electrical measurements made of these systems during their first development, was provided by the recent observations of Dr. J. J. Hauser and Dr. J. R. Patel on

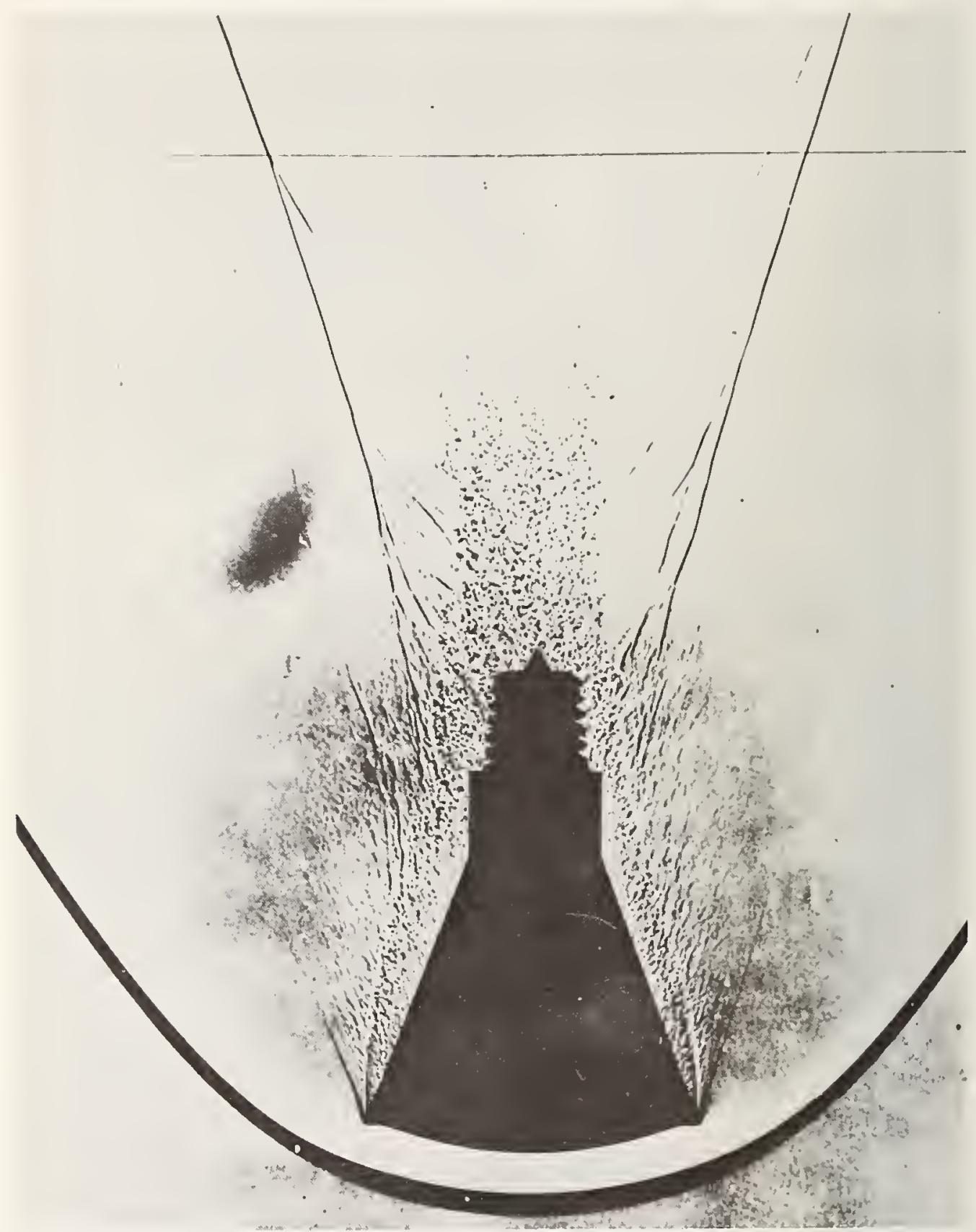

Figure 38. Shock waves and turbulence in laboratory tests of manned Mercury capsule protected by ablative heat shield. (Photo courtesy of NASA.) 
the conductivity of carbon-implanted diamond. As indicated in figure 39 , Hauser and Patel compared the temperature coefficient of dc conductivity of amorphous carbon films made by getter sputtering with those formed by

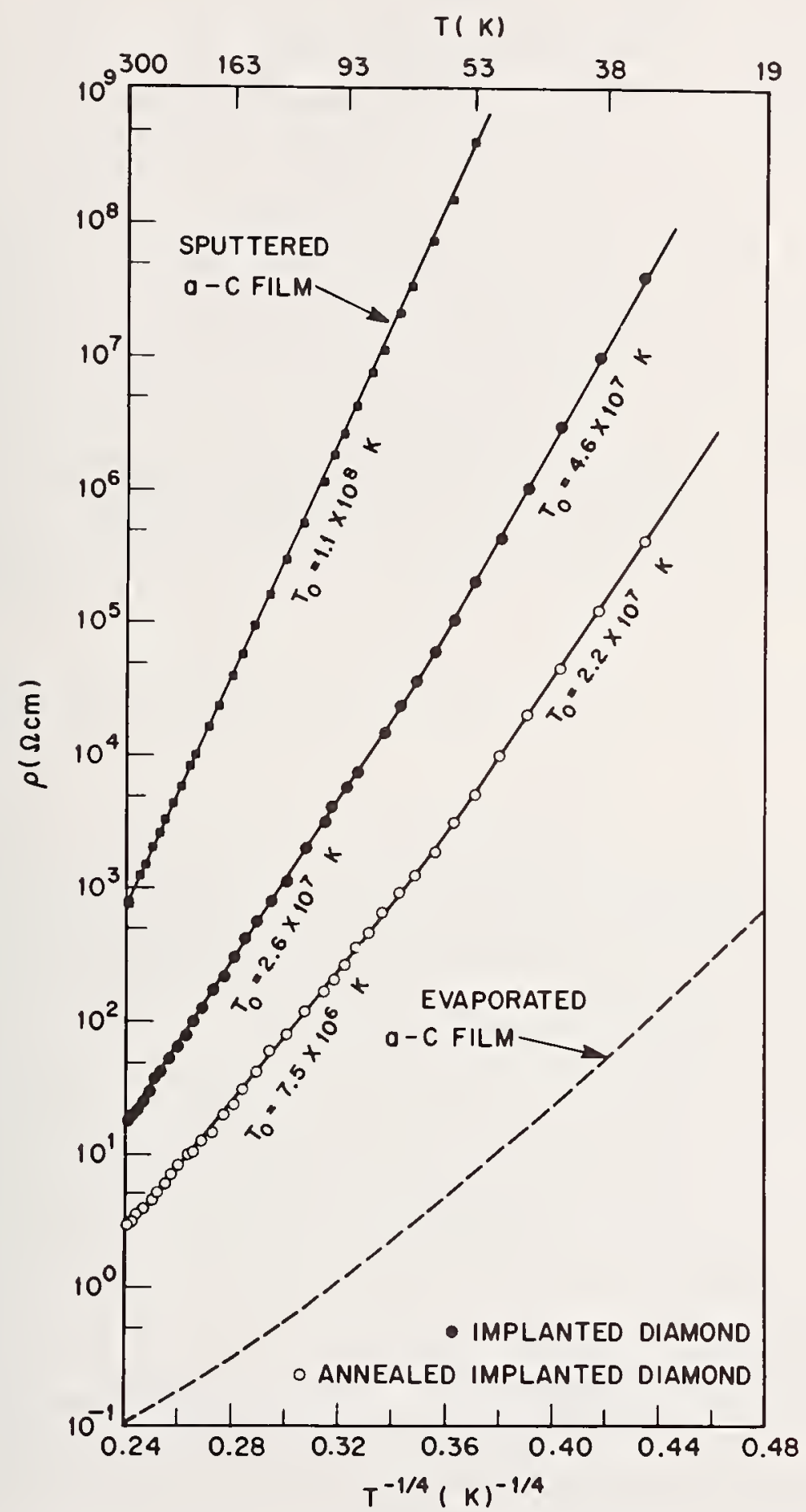

Figure 39. Temperature coefficient of de conductivity for amorphous carbon films, comparing films made by getter sputtering to those made by carbon ion implantation (in diamond). 
carbon ion bombardment of a diamond crystal, yielding an amorphous layer about 1000 angstroms thick and thus similar to the sputtered structure. The curves reflect an expression including the coefficient of exponential decay of localized electronic carrier states and the density of localized states at the Fermi level. The similarity of the slopes for the diamond-based and sputter-deposited amorphous carbon supports the general concept of mixed graphite-diamond bonds, which we deduced from other studies years ago. Optical transmission measurements also support this view. Short-range ordering by high-temperature annealing enhances the graphite content. But it is interesting that even when resistivity was reduced to $1.8 \times$ $10^{-3}$ ohm-centimeter at $300 \mathrm{~K}$, the annealed layers remained exceedingly hard. This supports the view that metallic conductivity indeed obtains when the localized states overlap, but there is still a preponderance of diamond bonds, yielding the remarkable mechanics of these polymers. Thus we have further experimental indications that the state of the solid is the same whether diamond bonds are added to graphite by polymer carbon formation and pyrolysis evaporation or sputtering, or whether graphite bonds are generated in diamond by bombardment implantation of carbon ions.

In addition to these rather specialized, predominantly carbon polymers, various other elemental polymer systems have drawn attention in recent times. Thus Dr. M. M. Lobes at Temple University and subsequently Dr. A. G. MacDiarmid at the University of Pennsylvania have produced polymeric sulphur nitride (polythiazyl), which shows many optical and electrical qualities of metals. Similarly, oriented fibers showing high anisotropy of electrical qualities have been produced. The phosphazene polymers have also had renewed attention recently, since the early work of Allcock in preparing poly(dichlorophosphazene). Likewise, organic conductors such as hexamethylene tetraselanofulvalenium-tetracyano-p-quinodimethanide have recently been studied by Cowan, Block and co-workers at Johns Hopkins University.

It would be unseemly to comment on synthetic materials or materials in general in this distinguished institution without referring to the many new techniques of measurement and characterization that are emerging. One of the most promising methods of analyzing complex shapes in terms of stress distribution and viscoelastic qualities is holographic projection of stress birefringence. Thus figure 40 shows the pattern around an incipient fracture center in a plastic, where a laser has generated highly sensitive interferometric evidence of stress concentrating beyond the elastic limit. Then in forming the hologram, as in figures 41 and 42 , we can reproduce an image of the real solid, as shown in figure 43 . In the figure, an injection-molded housing reveals subtleties of stress distribution and concentration of value to both the process and design engineer. 


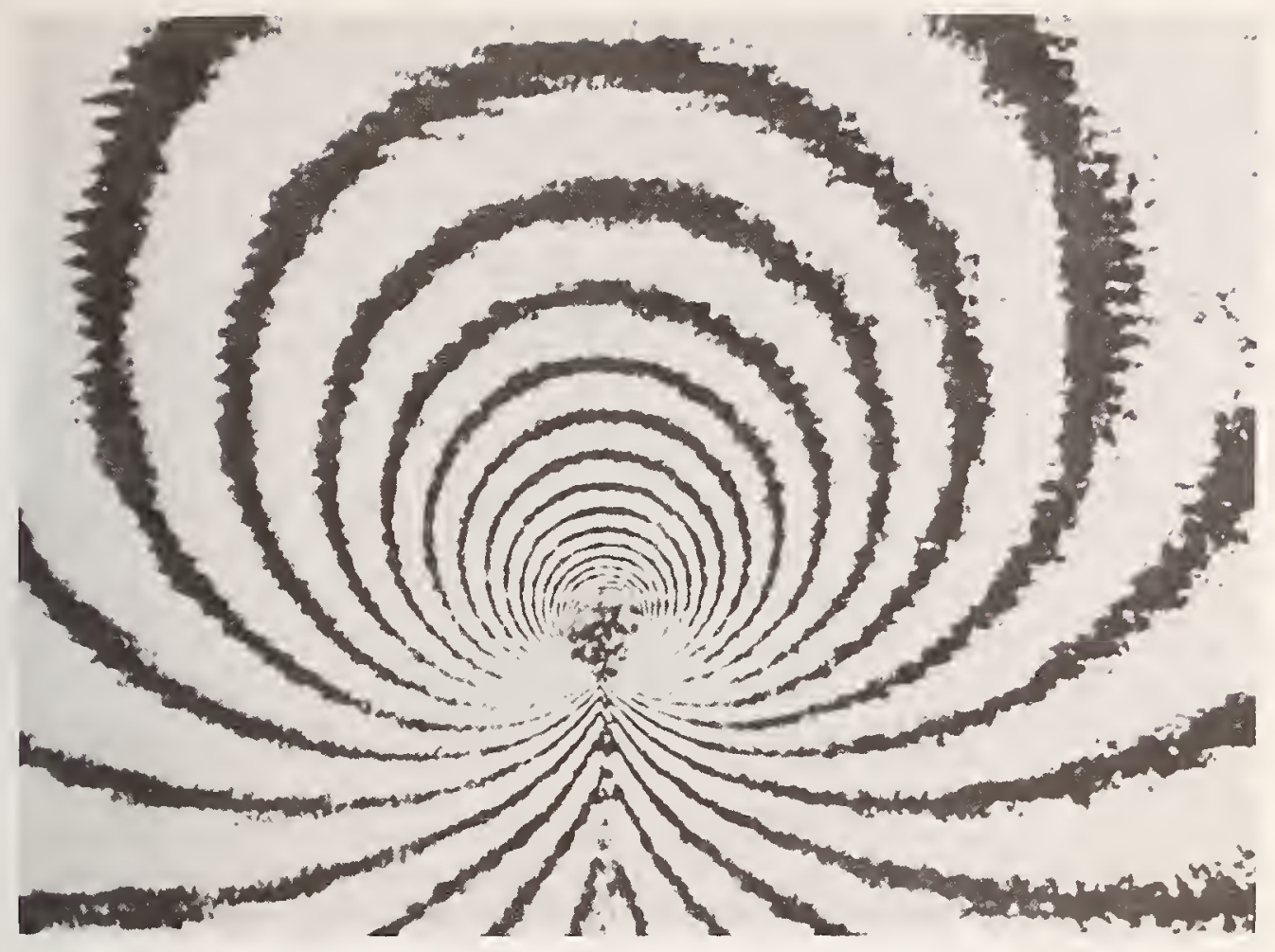

Figure 40. Incipient crack region in plastic is made visible by hologram. Stressed material is viewed through a superimposed hologram of the same material before stress was applied. Interference patterns are produced at points where reflected light from stressed surface is out of phase with the original sample.
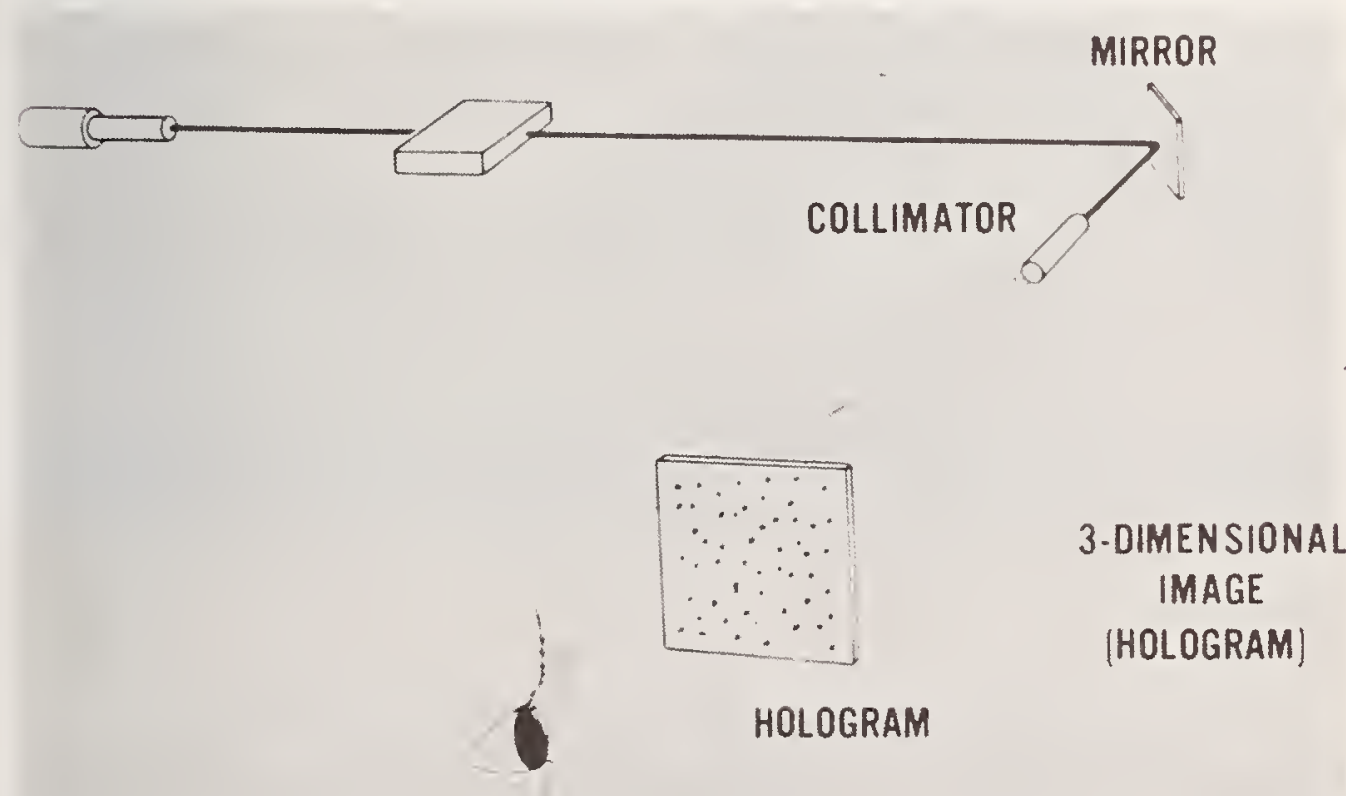

Figure 41. Viewing a hologram: The hologram is illuminated by a collimated beam of light, producing replicas of the wave fronts that were reflected from the original object. 


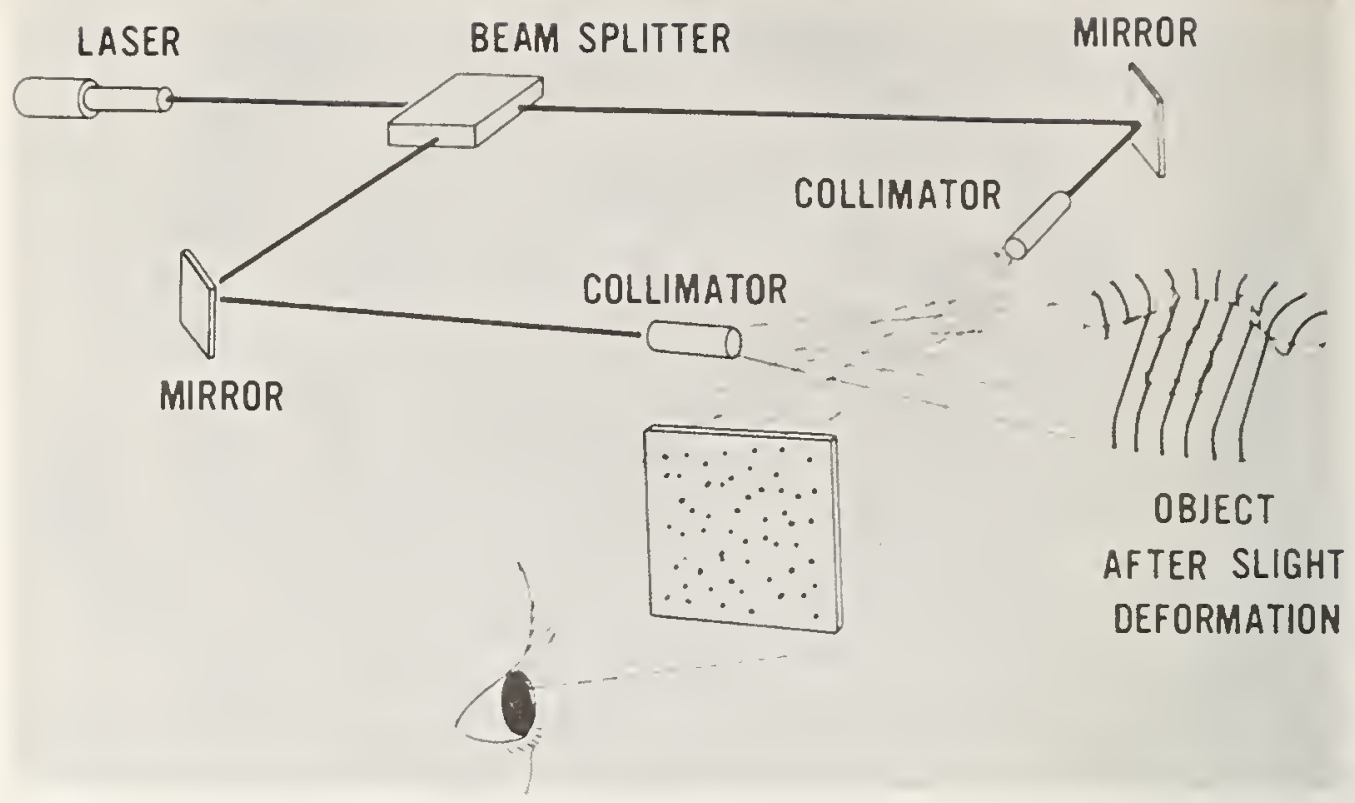

Figure 42. Recording a hologram: Laser light is separated into two beams by a beam splitter. One beam illuminates the object, the other serves as a reference. The photographic plate records the pattern of interference between reflected light from the object and the reference beam.

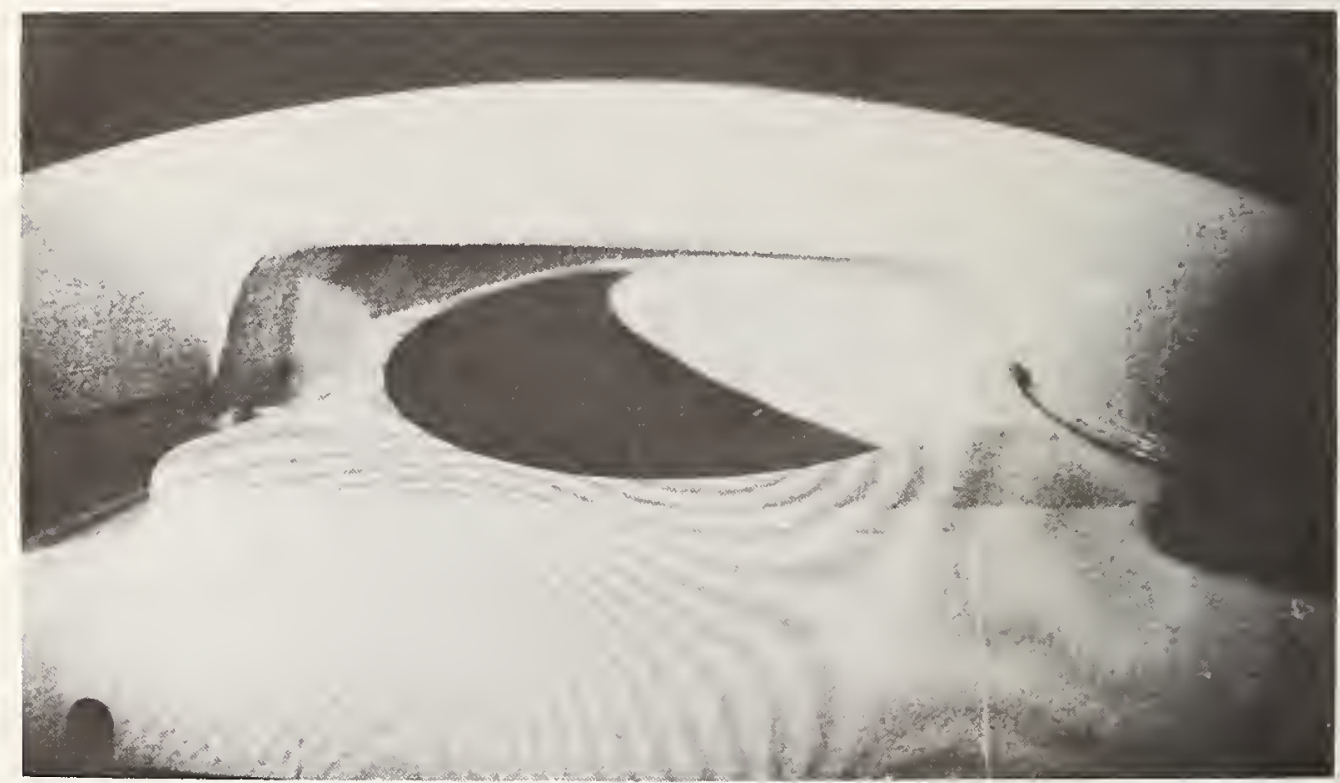

Figure 43. Hologram of a plastic telephone housing, revealing stress distribution and concentration.

As we have noted, the important characteristics of many inorganic and metallic solids, those factors determining their strength, elasticity, yield and general design utility, depend on the behavior of dislocation, vacancy and amorphous properties. Correspondingly, in synthetic organic systems, in polymer solids and rubbers, viscoelastic relaxation phenomena are the 
dominant mechanisms for physical, electrical, thermal and other properties. Thus, the past two decades have seen an elegant use of dynamic mechanics, and particularly of broad-spectrum shear and longitudinal wave characterizations, along with applications of polymer structure theory and computer modeling. These are giving a firm understanding of the principal variables in assuring desired rigidity, flexibility, high elasticity needed for yield quality, and the like.

In particular, the segmental motion under thermal agitation distinguishing the rubbery from the glassy, or often rigid, crystalline states has been well established. Thus, in one of the most widely studied rubbery systems, polyisobutylene, figure 44 shows a temperature dependence of relaxation frequency above and below glassy transition calculated by a relatively satisfactory model. The engaging feature is that measurements based on mechanical waves, electrical waves (responsive because of polar impurity groups in this and virtually all other polymer chains examined) and nuclear magnetic resonance measurements from various proton relax-

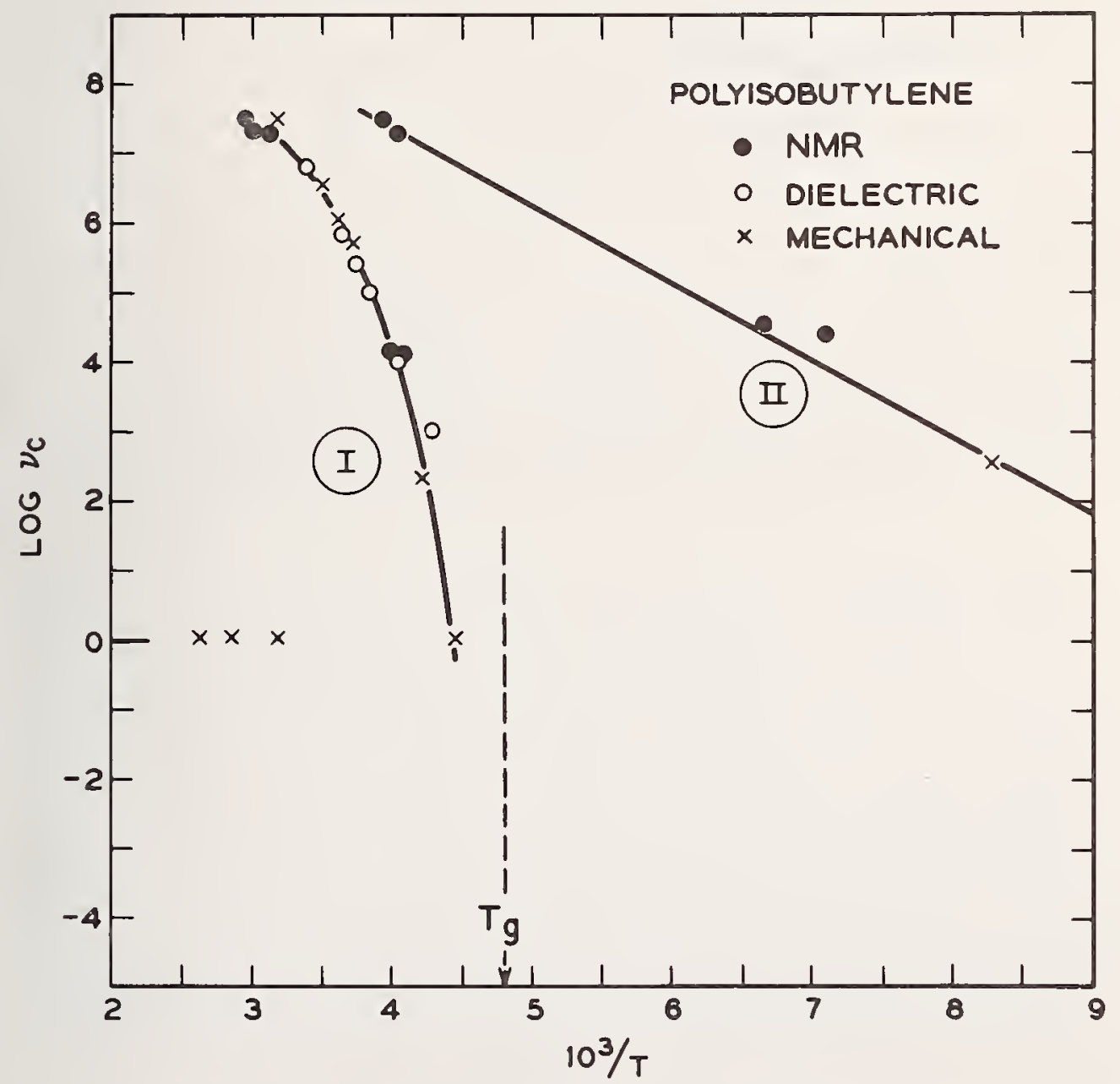

Figure 44. Temperature dependence of relaxation frequency in polyisobutylene, showing agreement of nuclear magnetic resonance, dielectric, and mechanical measurements. 
ations coincide elegantly. Similarly, the typical crystalline polymer polypropylene, in figure 45 , displays the same consistency, as do the polar copolymer of fluoroethylene and ethylene in figure 46 and the completely substituted chain polytetrafluoroethylene in figure 47 . Naturally, such indication of consistent structural response to external mechanical and electrical fields as a result of thermal agitation has been already widely applied in engineering. We control, for example, various extrusion and molding

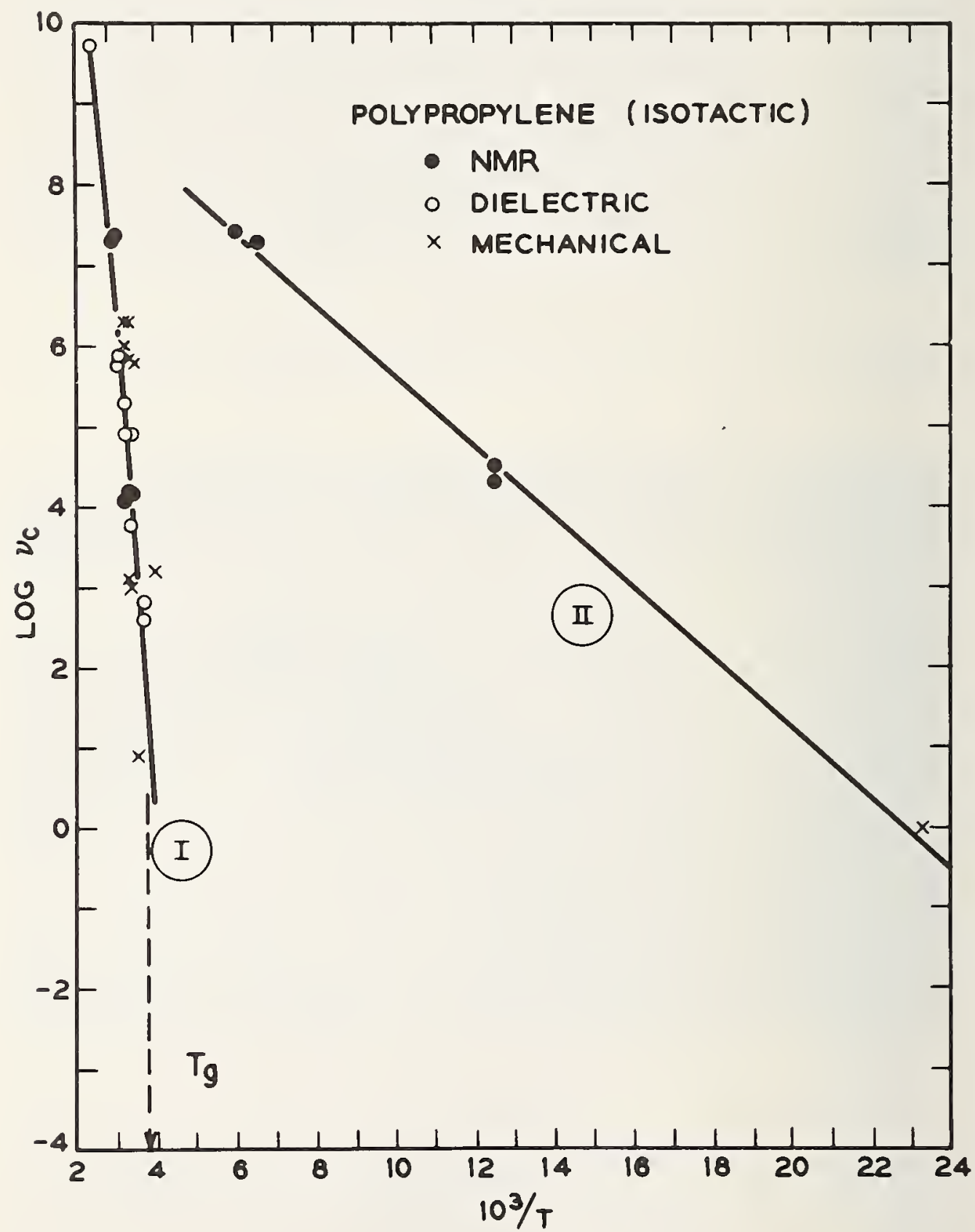

Figure 45. Temperature dependence of relaxation frequency in polypropylene, showing agreement of nuclear magnetic resonance, dielectric, and mechanical measurements. 


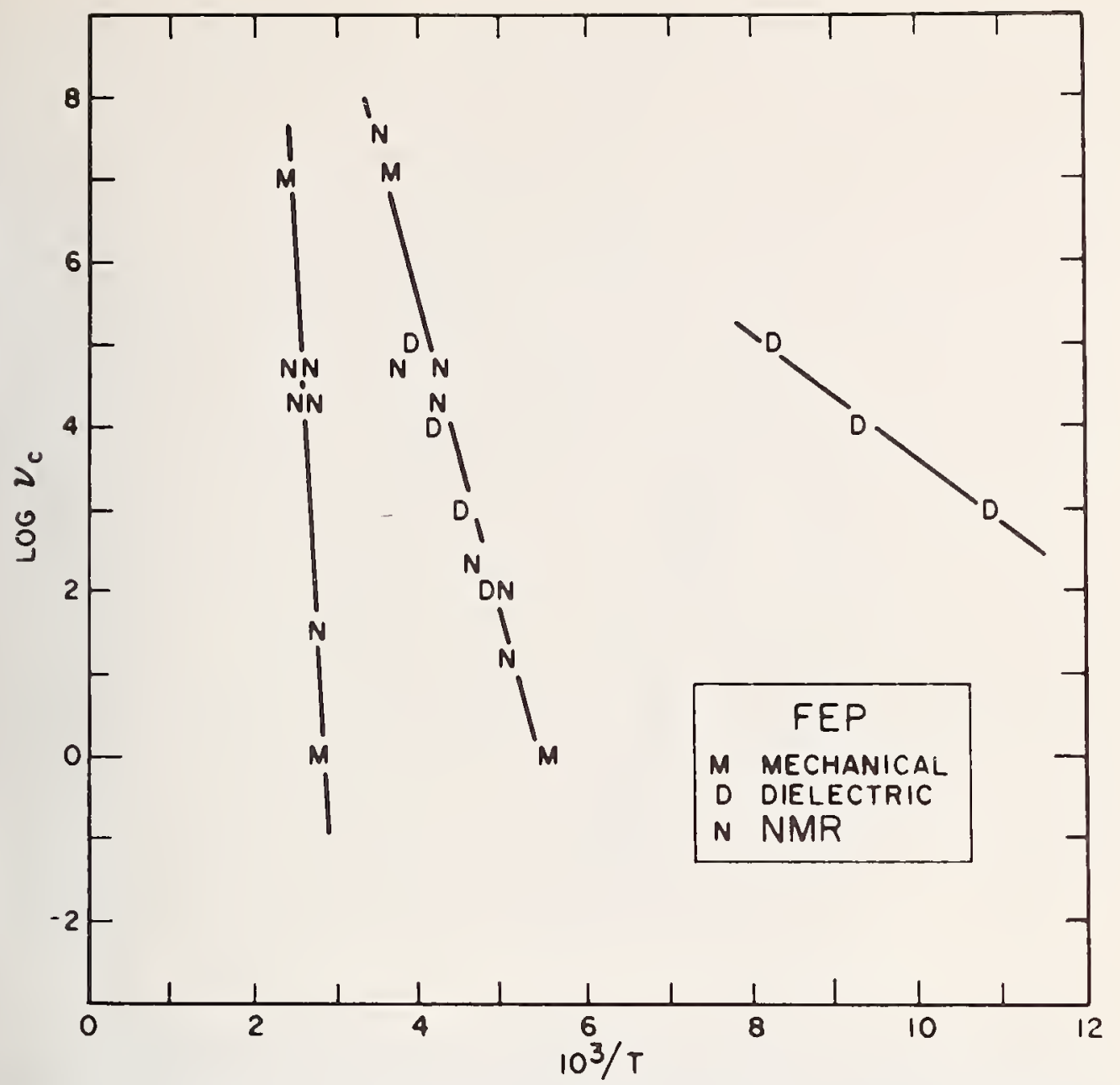

Figure 46. Temperature dependence of relaxation frequency in fluorinated ethylenepropylene (FEP), showing agreement of nuclear magnetic resonance, dielectric, and mechanical measurements.

processes by calculating from these relaxation times the necessary compliance of the plastic or rubber as it is cooled in the final form.

Knowledge of this sort has helped revolutionize such energy-demanding operations as the processing of rubbery structures, an enormous element in the materials industry, the basis for all vehicle tires and power links. Figure 48 exhibits the dramatic results of an old modification of the structure of rubber molecules through conversion of chains in locally netted configuration by the method of microgel synthesis, discovered in latex polymerization more than 30 years ago. New applications of this technique are still being made through molecular control of the latex particle, and even natural rubber has been vastly improved by post-treatment of its molecules before coagulation. As seen in the figure, the use of microgelnetted molecules so alters the relaxation process during high-speed extrusion that compliant and stress-free extrudates are readily obtained. These 


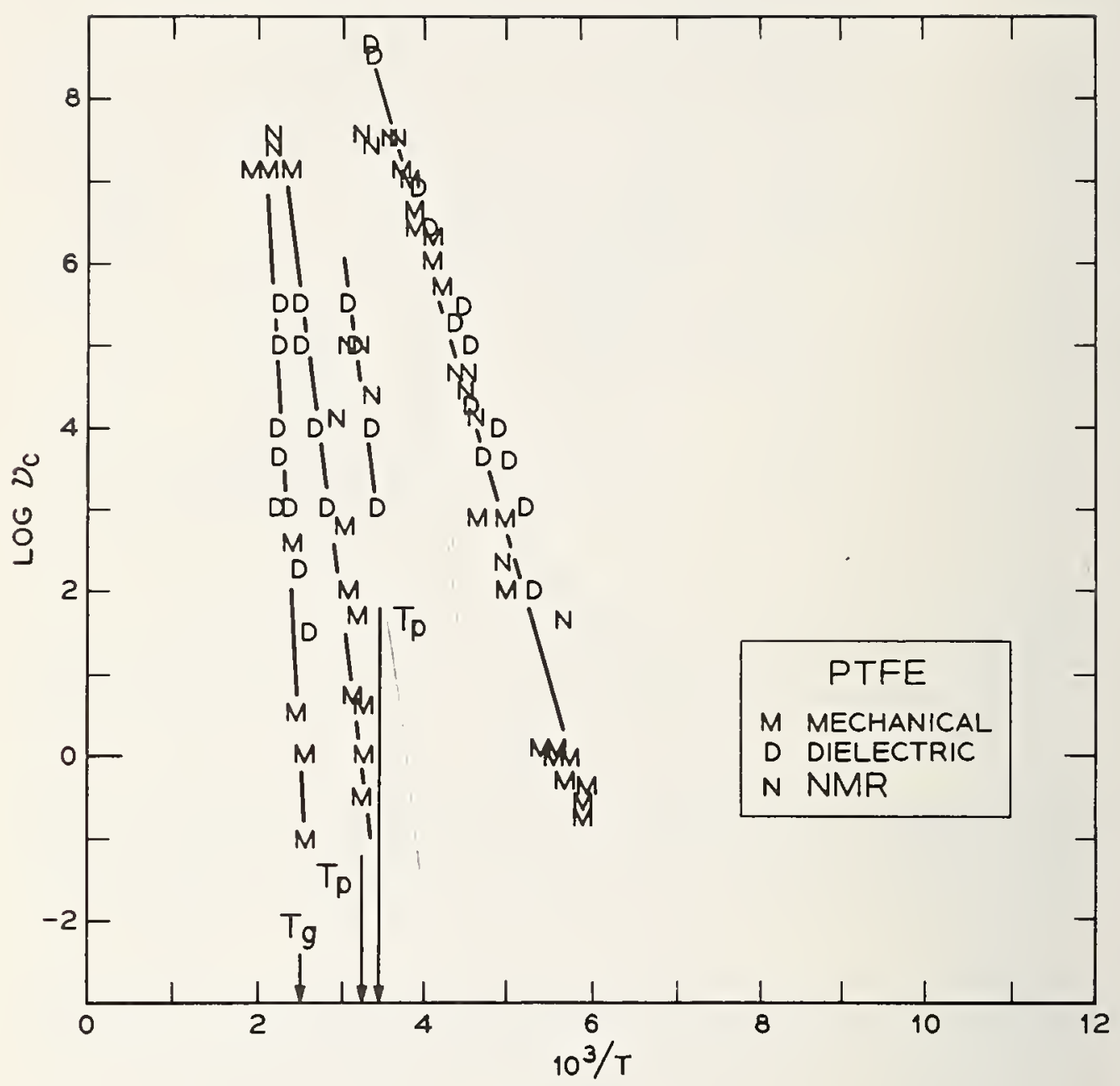

Figure 47. Temperature dependence of relaxation frequency in polytetrafluoroethylene (PTFE), showing agreement of nuclear magnetic resonance, dielectric, and mechanical measurements. 
contrast sharply with the highly distorted and subsequently relaxing volumes resulting from conventional milling and processing.

Of course there are also many polymer systems whose qualities are dominated by high polarity and by dipole bonding and interaction. This is especially true in fiber-forming systems. Here again we have a thorough basis for process and physical control through detailed structural studies, such as those of the dipole layer association, shown for typical polyamides in figure 49. Also being revealed are the structures of polymer blends and especially of the important emerging polymer metal composites and polymer adhesive layers. Figure 50 shows the influence of a copper substrate on polyethylene crystallization. It gives evidence, micrographically, of a special oriented layer of lamellae at the polyethylene/copper oxide interface. This forms before the typical spherulite structure appears farther on in the polymer solid. Precise control of this process was vital in the production of many transatlantic submarine cables, where phenomenal pressures and extreme environmental demands were successfully met with copper-polyethylene high-pressure seals. These work under circumstances where only a few years ago polyethylene seemed the most unpromising adhesive, which could never be trusted at extreme seabed conditions. The latest, exceedingly high-capacity transatlantic cable, TAT-6, presently being installed, outpaces all satellite communications in economy and fidelity; it depends much on this physical structure.

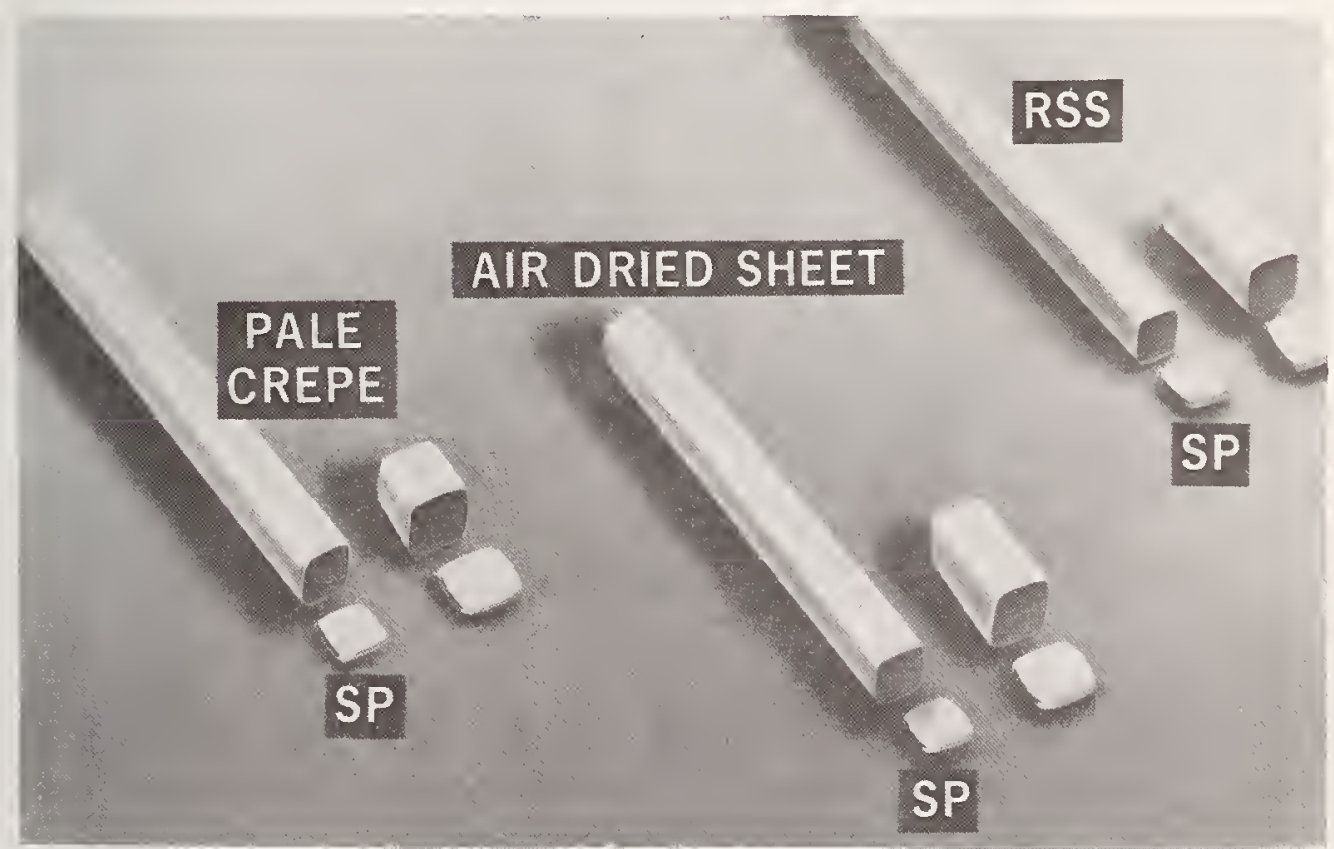

Figure 48. Extrusion of three major types of natural rubber. Special processing (SP) through partial cross-linking alters relaxation of rubber so that extrudate retains size of die more closely than rubber produced by conventional processing. 


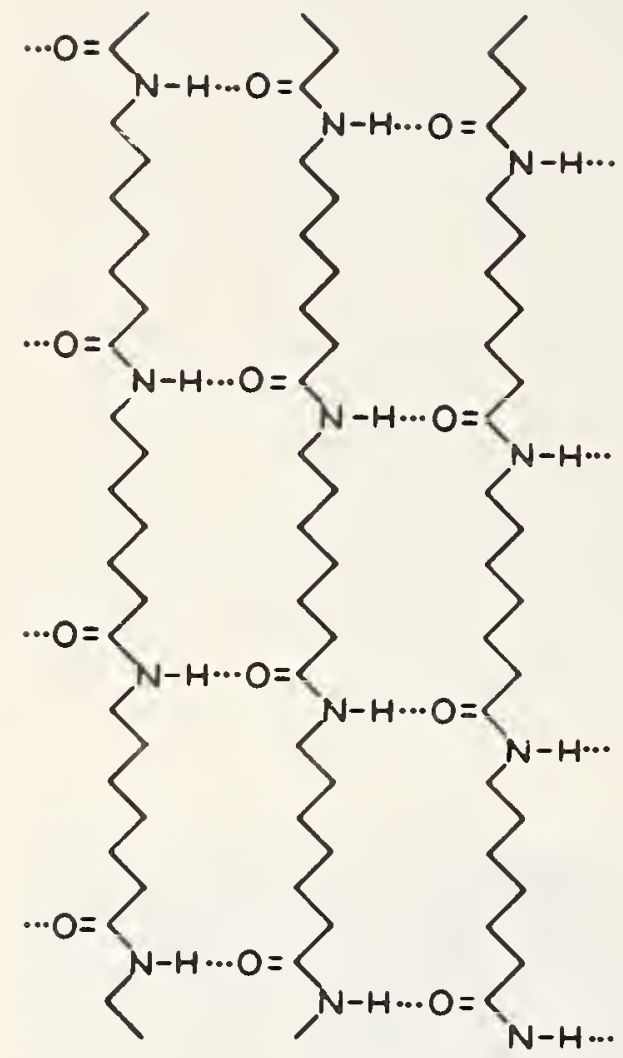

(a)

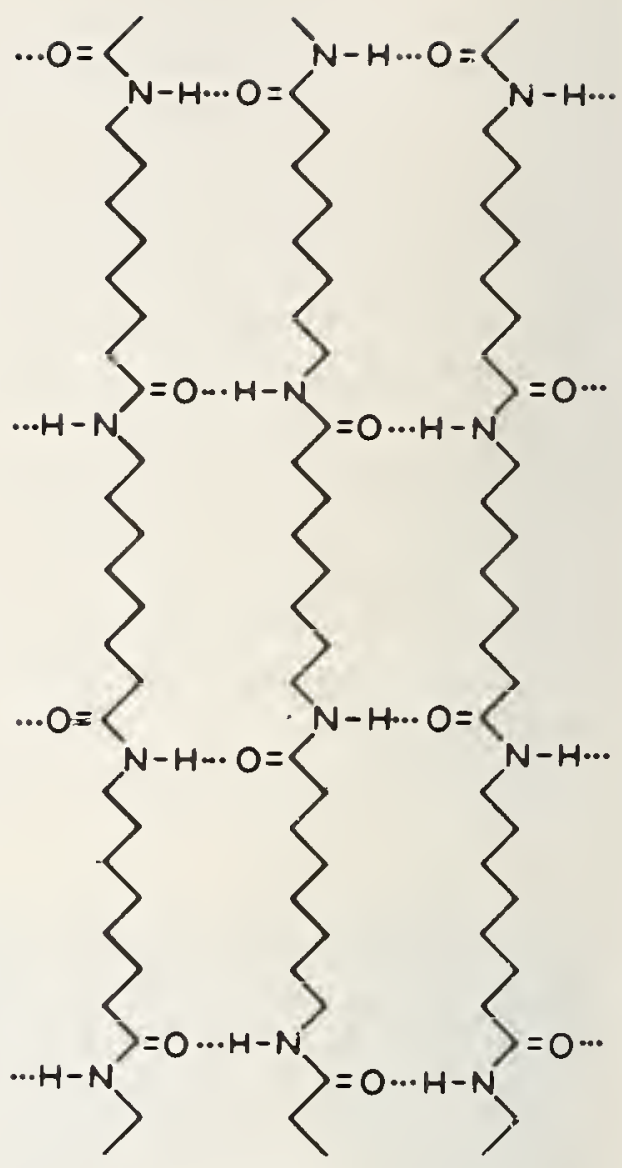

(b)

Figure 49. Dipole layer association: structure of polyamide chains made from (a) odd-numbered $\omega$-acids or even-numbered di (amines and acids) or (b) even-numbered $\omega$-amino acids or odd-numbered di (amines and acids). 


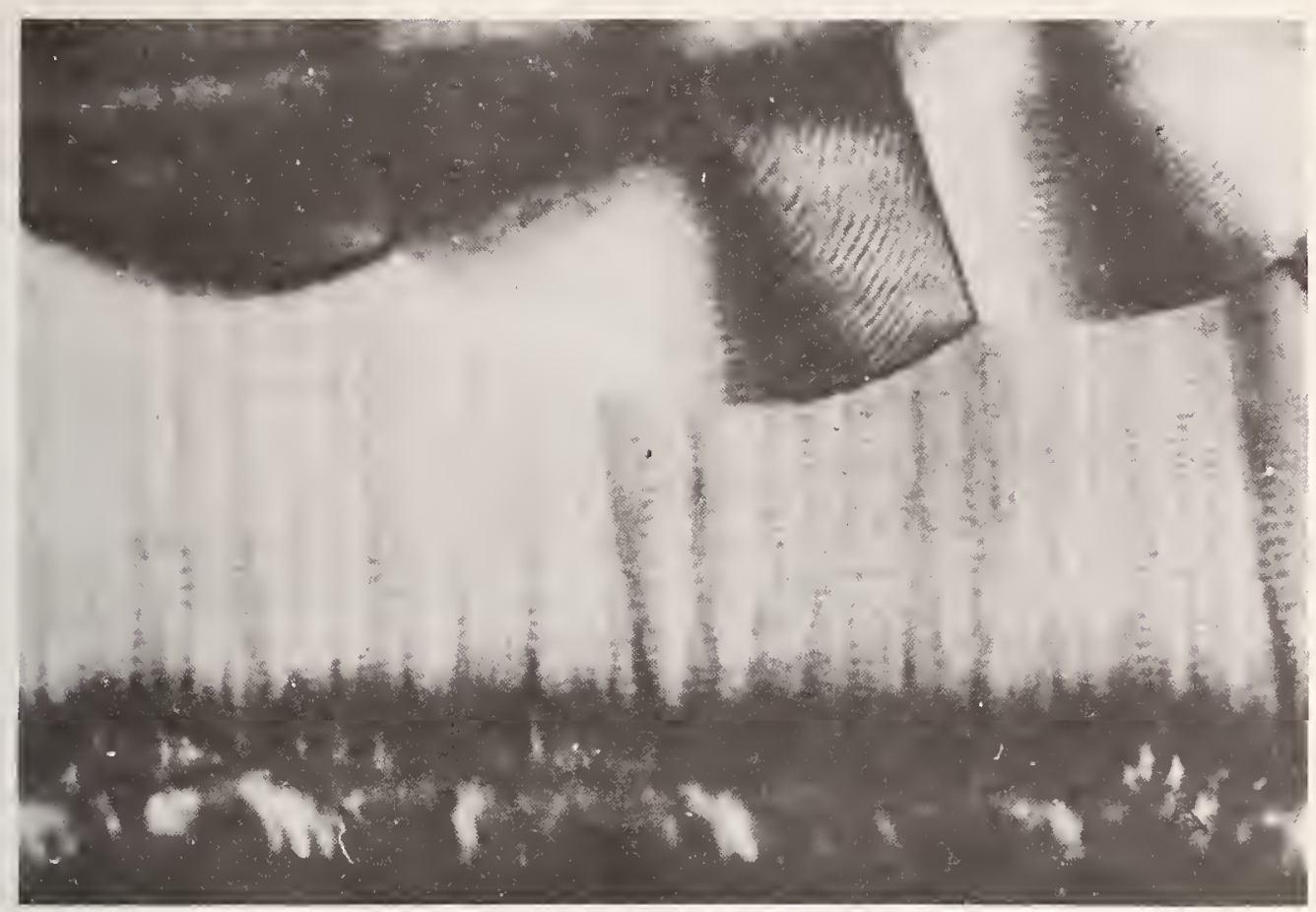

Figure 50. Polarized light micrograph of polyethylene crystallized on heavily oxidized copper sheet shows highly oriented lamellae separating the polymer/metal junction (dark portion on right) from the usual spherulite crystalline structure (left) deeper inside.

As a matter of fact, the analogies between microcrystalline polymers and certain aspects of metal behavior are increasingly important. Some decades ago, we found behavior that has become vital to the development of polyethylene for containers, tubes, pipes and other stress-enduring applications. This is that biaxial stressing of the solid produces drastically different stress/strain qualities than had been assumed by simple application of uniaxial testing. The differences were at that time also not fully understood in metals. But in our original investigation of the crystalline textural responses to this stressing, we found that complex gliding in both metals and polymers induced stresses such that, in the industrial production of polyethylene for instance, shifting the molecular weight distribution yielded a bulk material more capable of sustaining these forces. This meant, of course, changes in both the nature and cohesion of the crystallites. As shown in figure 51, these methods are now producing polyolefins with phenomenal strength, endurance, and resistance to mechanical degradation by surface agents and environmental influences under biaxial stressing to which they were originally intensely vulnerable.

We have seen that the noncrystalline state can apply broadly to metals and inorganic systems, as well as to polymers. We find new horizons in view with respect to the most classic noncrystalline solid, glass. Despite superb progress at mid-century for the production of glass fibers, for exam- 


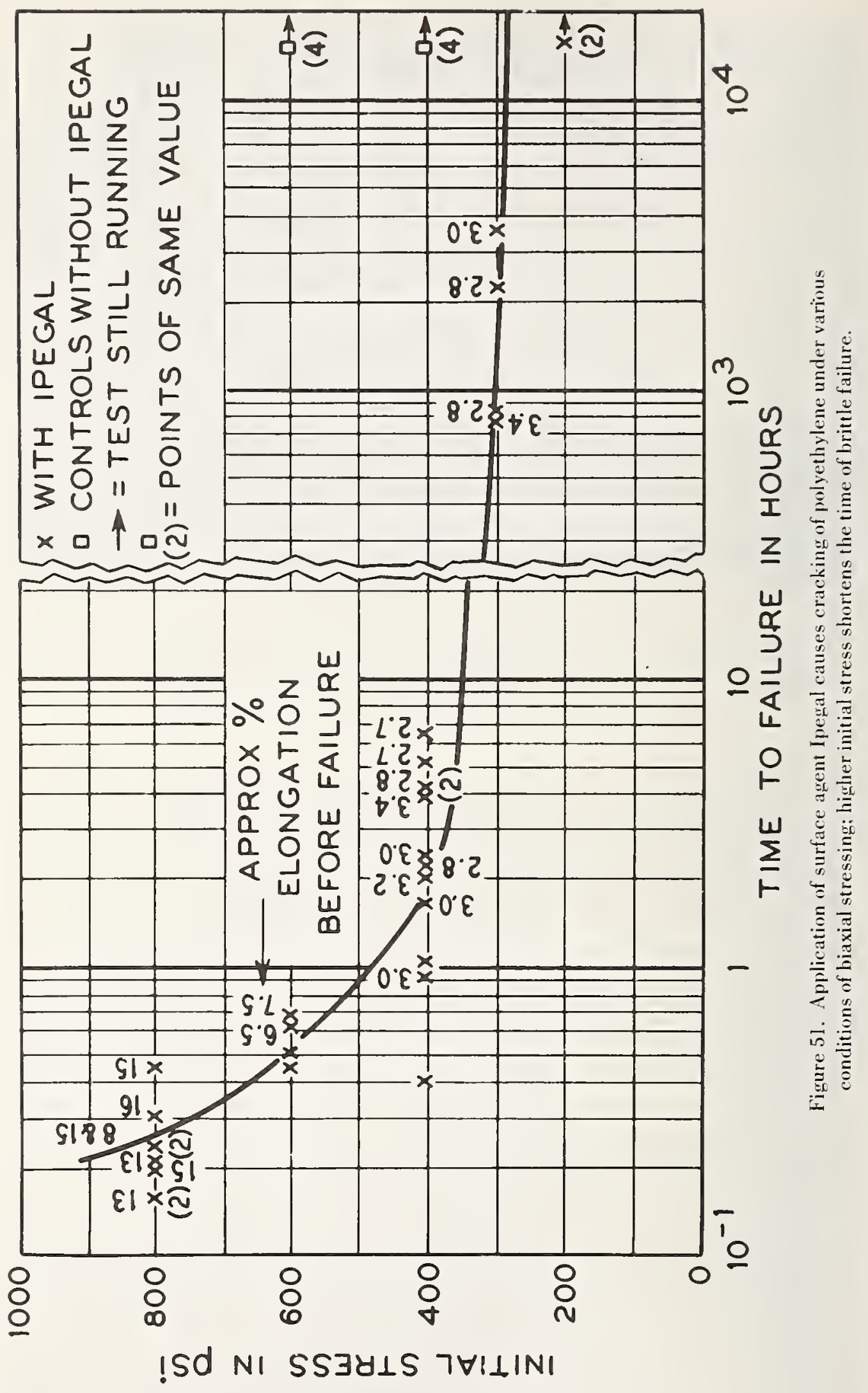


ple, which played a vital role in the industry of polymer composites, we are now proceeding into a new era of glass fibers, synthesized for their optical perfection but also showing mechanical and other physical properties of high interest. Figure 52 shows the methods used to produce such materials; figure 53 shows the products, with optical clarity nearly a thousand times that of the best glass lenses ever made before. Figure 54 indicates the construction of these fibers, which produce, as shown in figure 55, a unique optical medium, yielding in figure 56 historic efficiency of light transport, with loss of less than 3 decibels per kilometer.

You already know of the programs at Bell Labs and elsewhere applying these systems to new urban and local communications, but it is likely they will have an impact on all information handling and signal processing, as well as on new instrumentation. As shown in figure 57, lightguiding is indeed a new frontier, but it will be associated with equally extraordinary materials used in films, prisms, couplers and the like. As shown in figure 58 , these are leading to new optical circuitry, or integrated optics, which will have its part in science technology and industry in the years to come, as the thin film electronics of semiconductors is presently revolutionizing that field.

But on this occasion, devoted to the future as well as to saluting the past, comments on polymer material should conclude only with emphasis on the challenges ahead. These rely still, as they have in the past, on matching the functional elegance of natural systems, through synthesis and understanding. We have come a long way in fibers and plastics, in mechanical and electrical-chemical terms. However, in such features as efficiency of information handling as well as physical-chemical versatility, such arenas as the polynucleotides shown in figure 59 lie still beyond our understanding. Nevertheless, as diagrammed in figure 60, the beautiful helical structures and subtle interactions of the polar bases do connect with our emerging knowledge of simpler synthetic systems and give high promise of leadingr us still deeper into understanding of organic matter. 

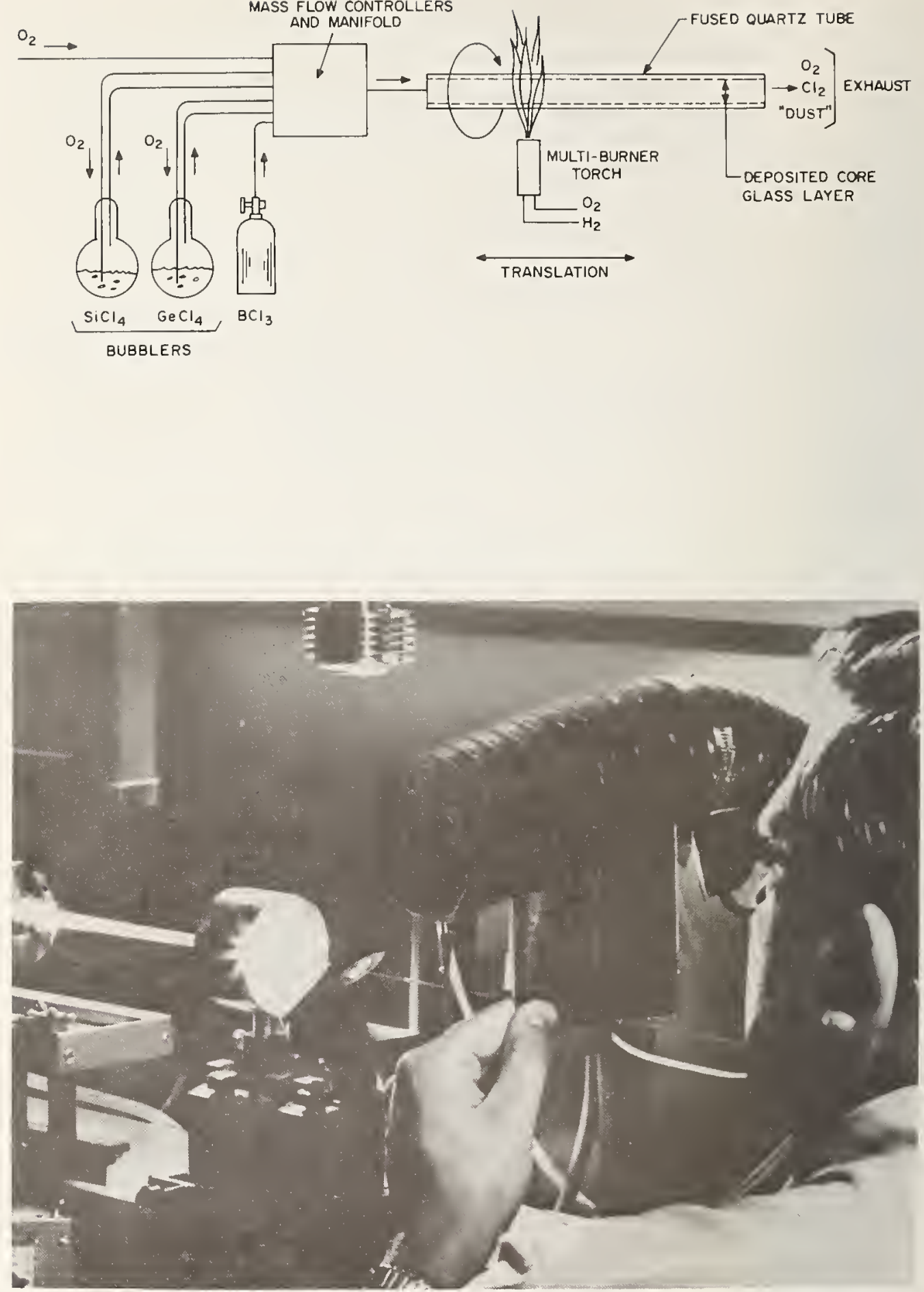

Figure 52. Production of low-loss glass fibers. (a) Modified chemical vapor deposition method of producing fibers with high silica content. Reactant gases, fed through a fused quartz tube, are heated to allow glass-forming oxides to be deposited on the tube's inner surface The tube is then collapsed into a solid rod, which can be drawn into hair-thin fibers by heating (b) with carbon dioxide laser. 


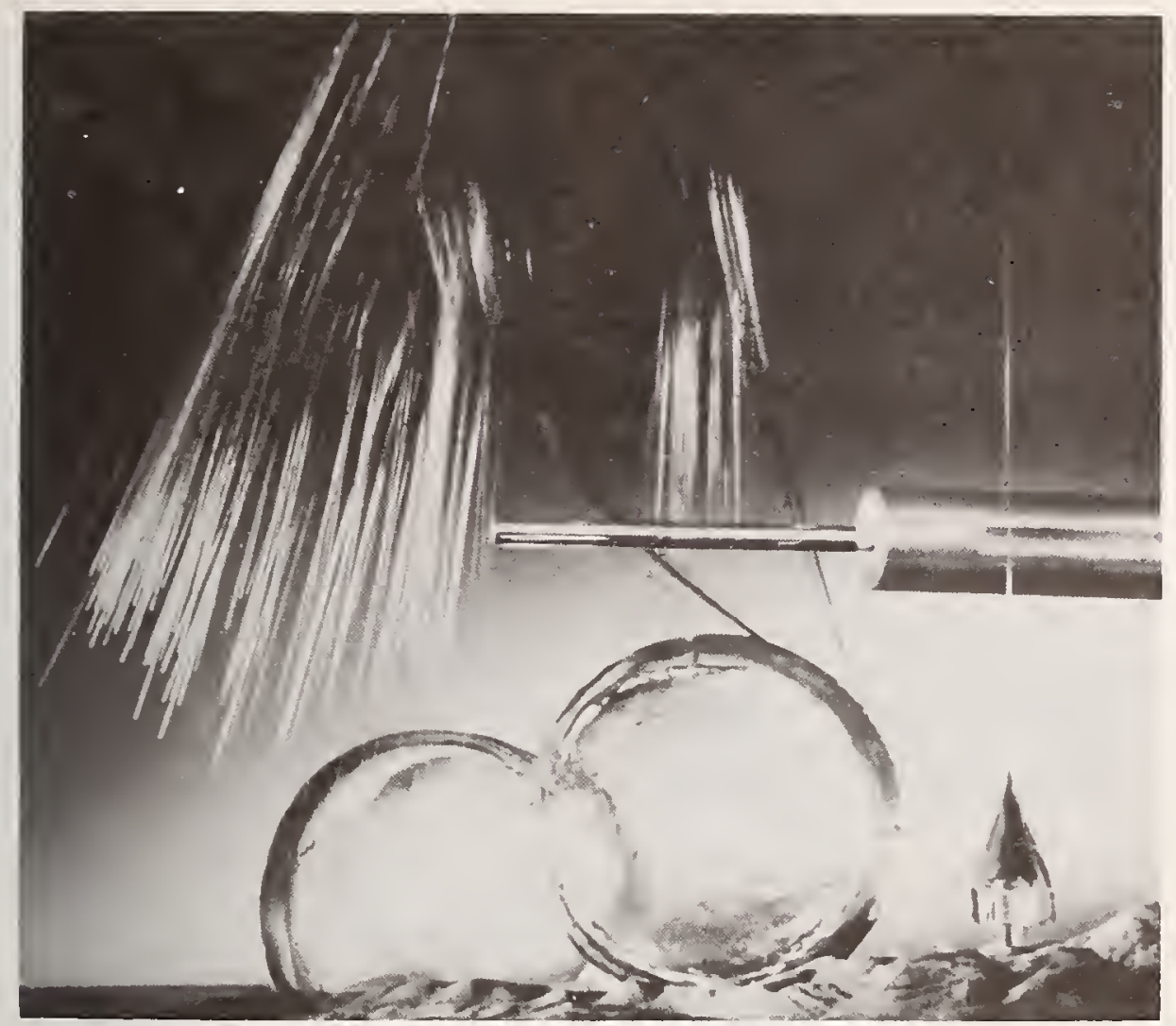

Figure 53. Preparation of glass fibers: raw materials and finished fibers.

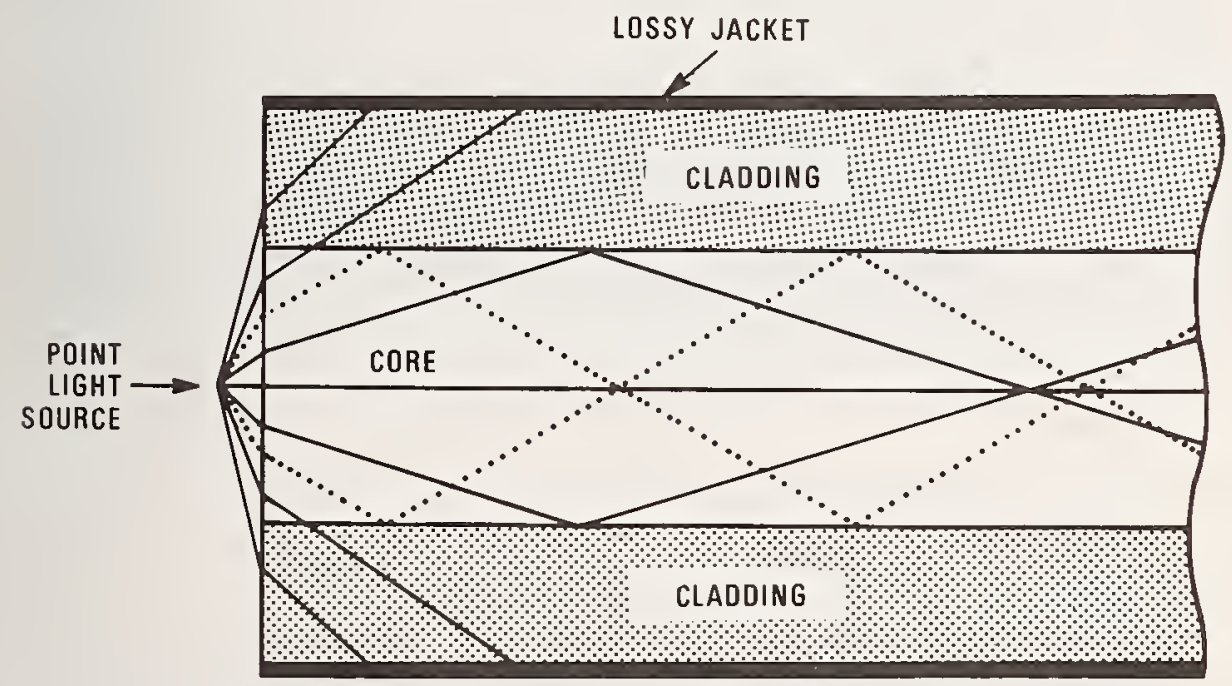

Figure 54. Fiber lightguide consists of two coaxial glasses surrounded by a light-absorbing jacket. The multimode fiber shown can transmit incoherent light. Some rays, not trapped in the core, are absorbed by the jacket; other rays are trapped and forced to propagate within the core. 

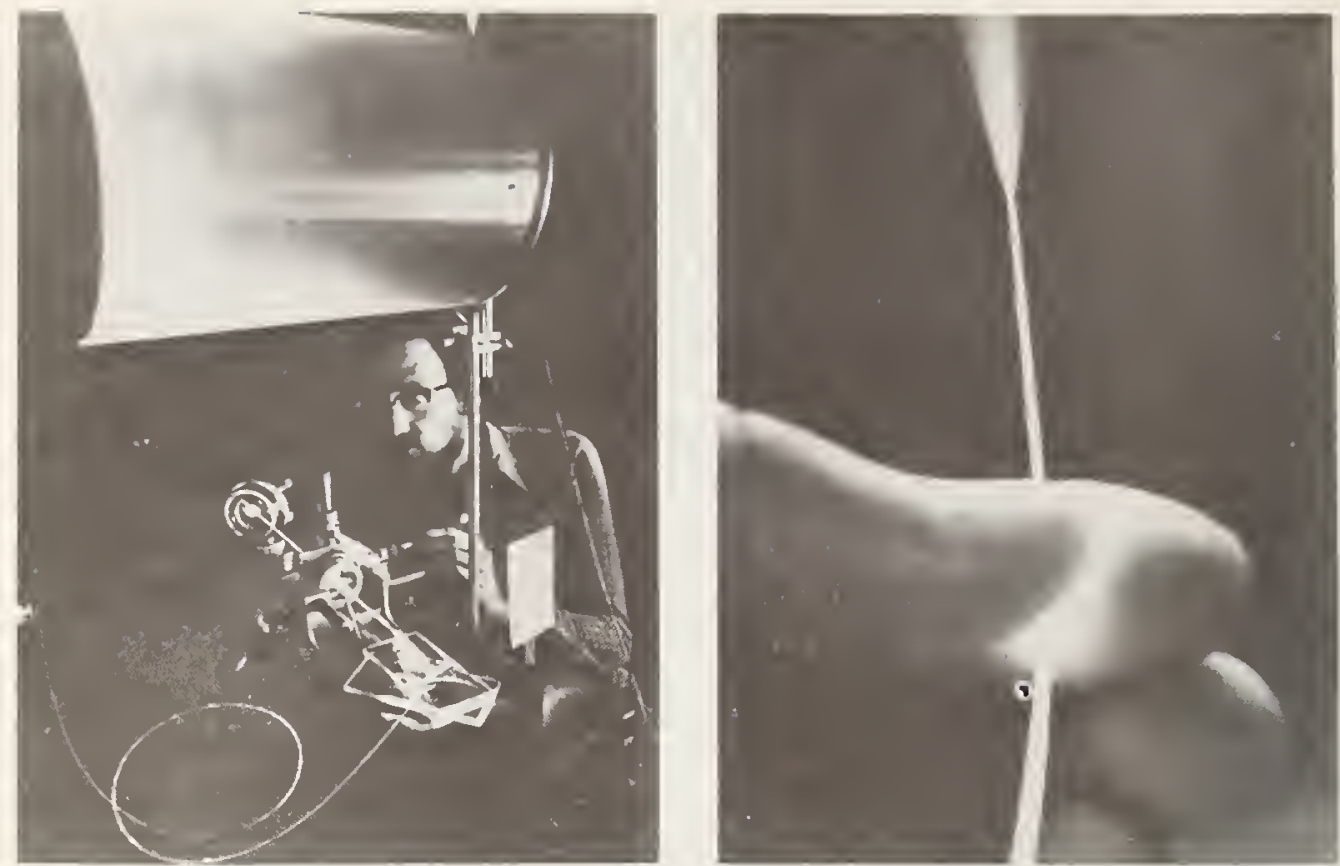

Fiqure 55. Glass fibers provide a unique medium for transmitting light signals. Laser light enters lightguide in center of photo (left), travels half a mile on drum, then illuminates card. Light emanating from light guide (right) has traveled half a mile on fiber.

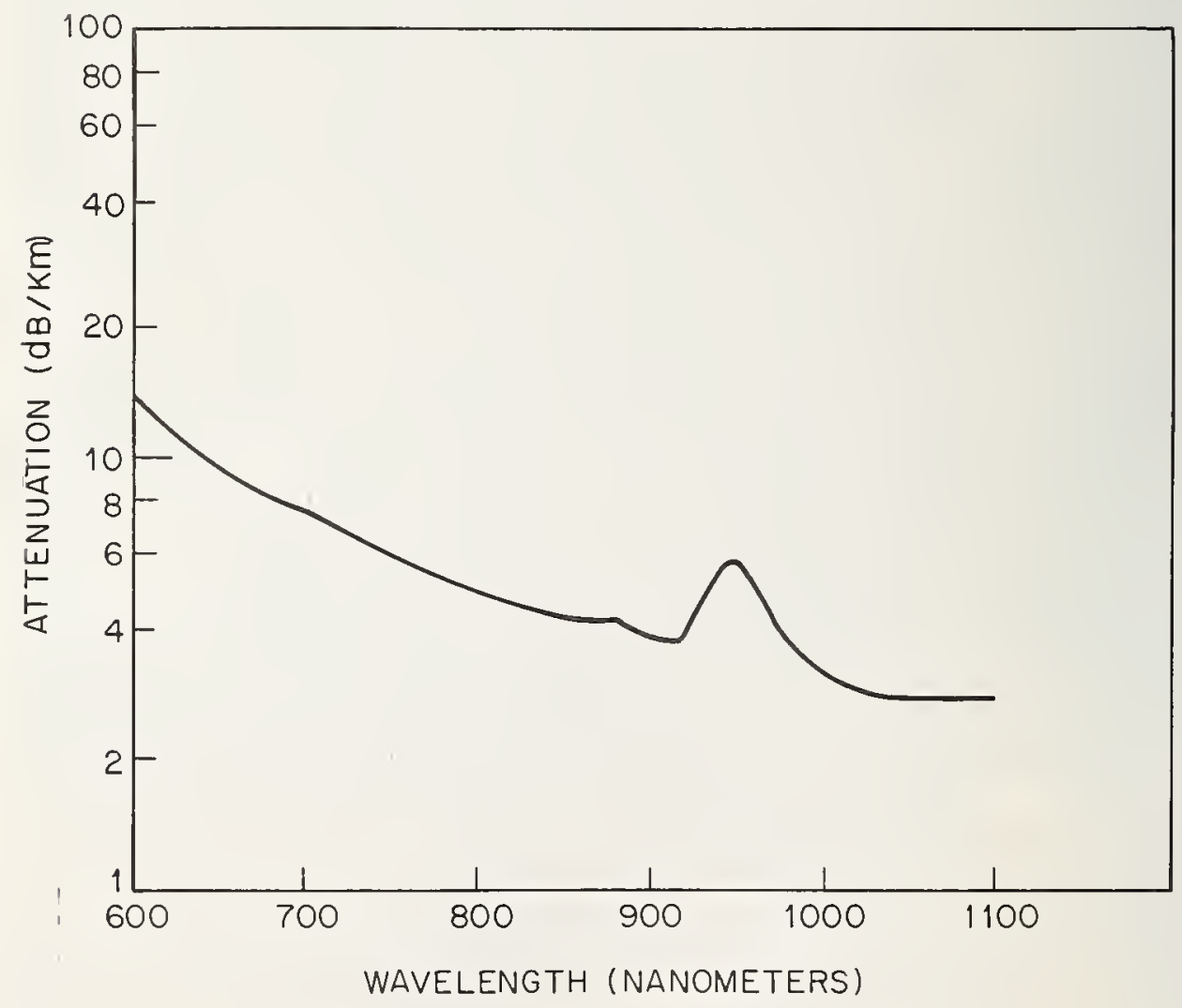

Figure 56. Efficiency of glass fiber light guides. 


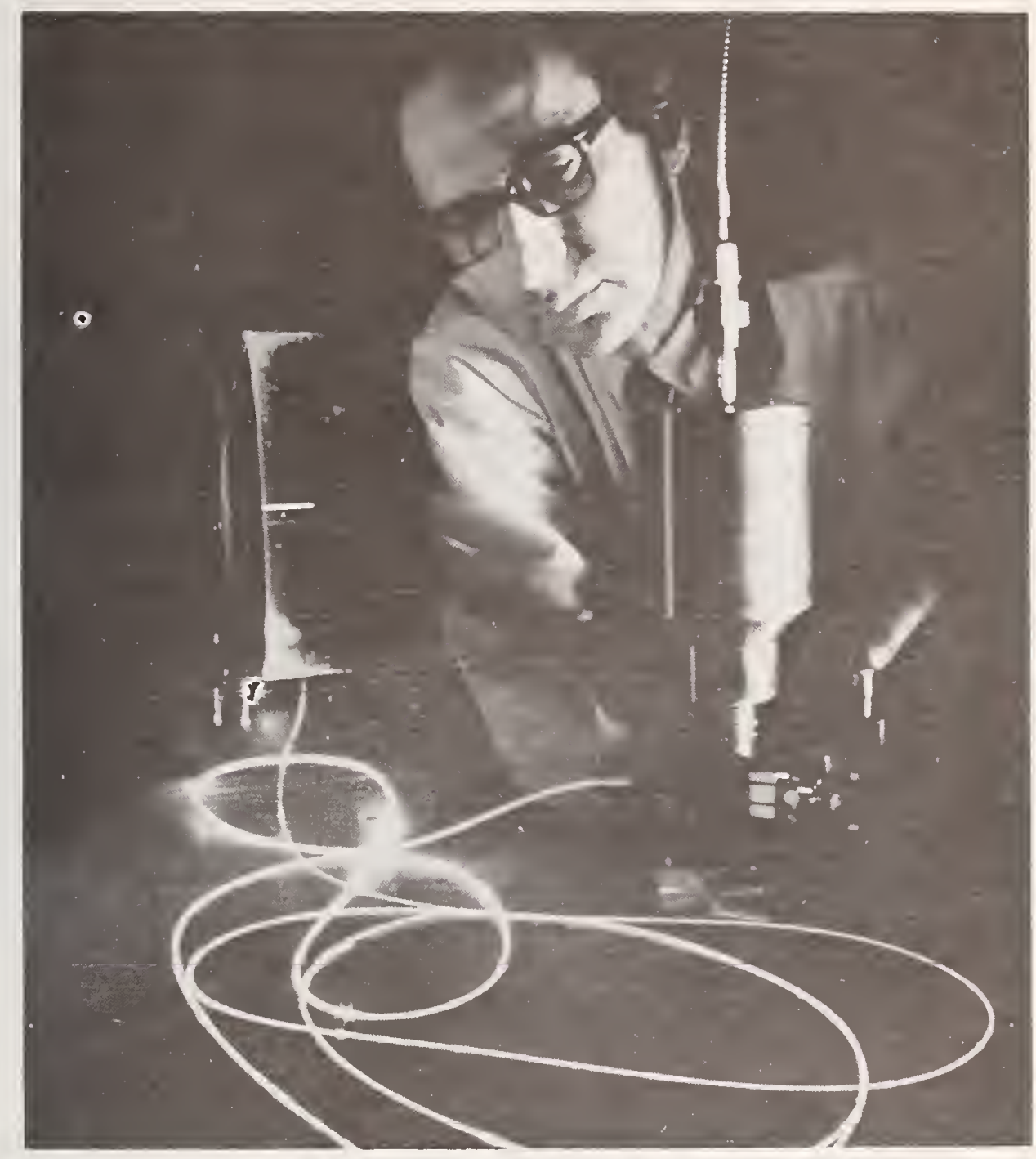

Figure 57. Glass lightguides like this will someday carry telephone calls on lightwaves. One lightguide may transmit a few calls within big cities or several thousand calls over long distances. 


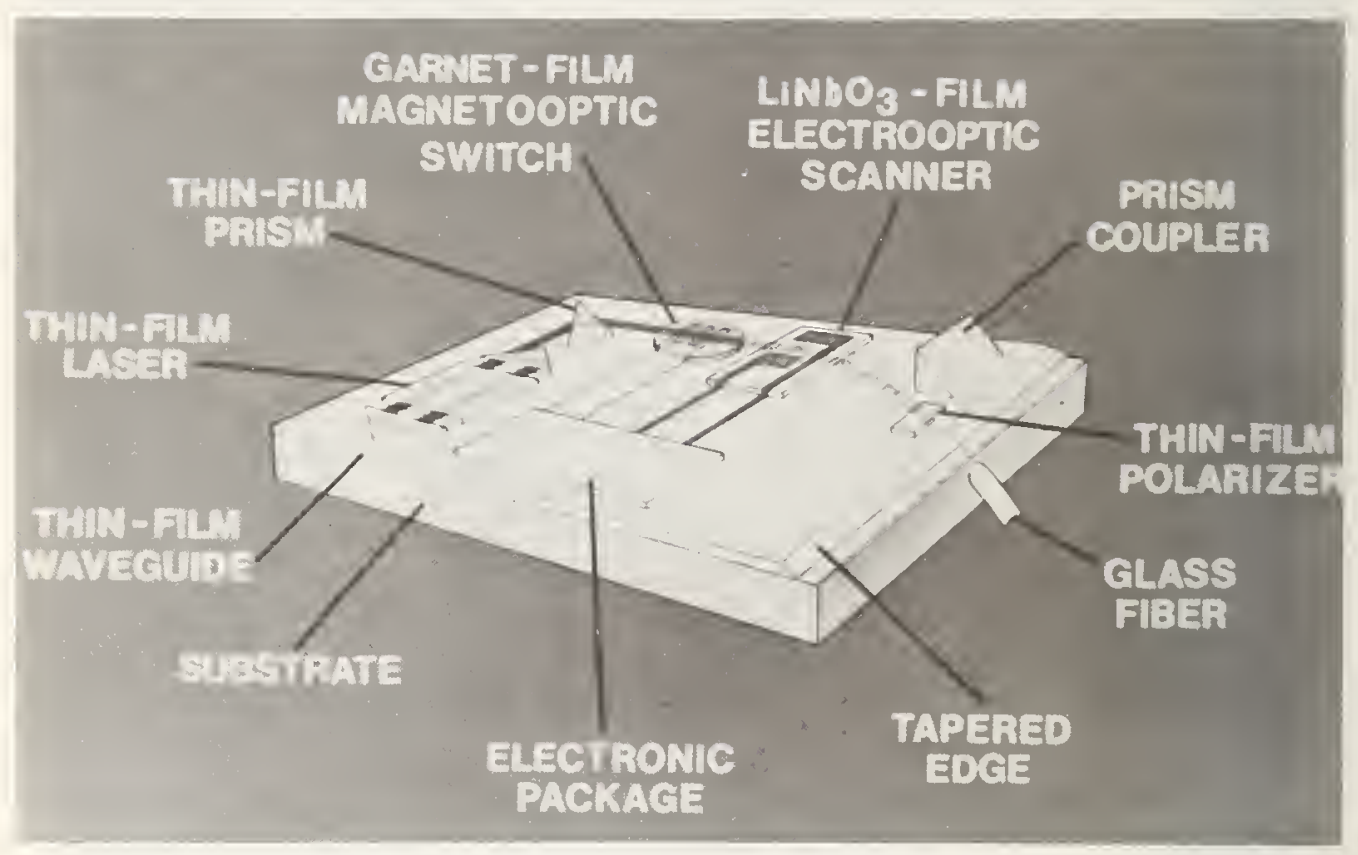

Figure 58. Proposed hybrid integrated optical circuit for generating and transmitting lightwave signals. It combines discrete components such as laser, switch and glass lightguides in one package. 


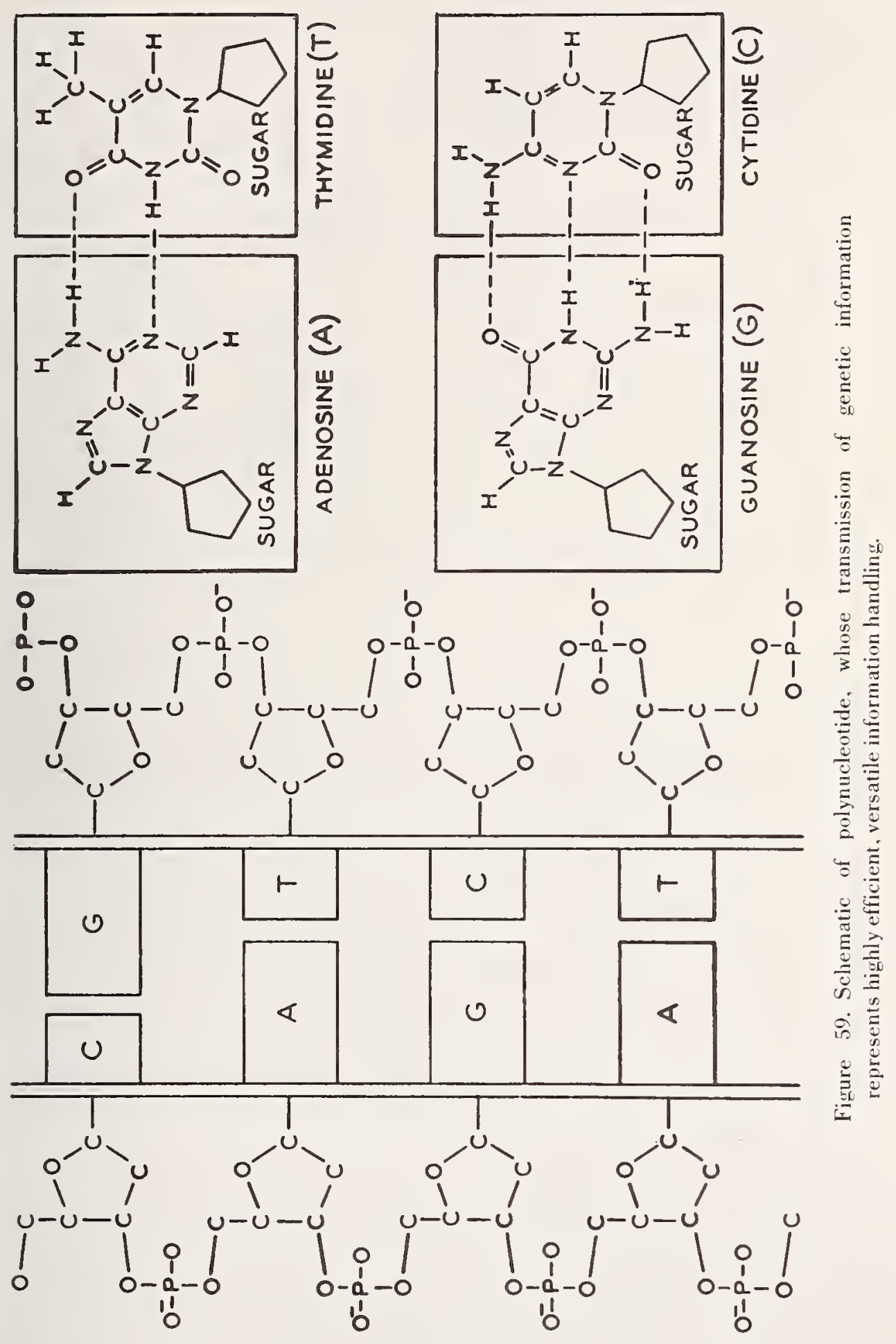



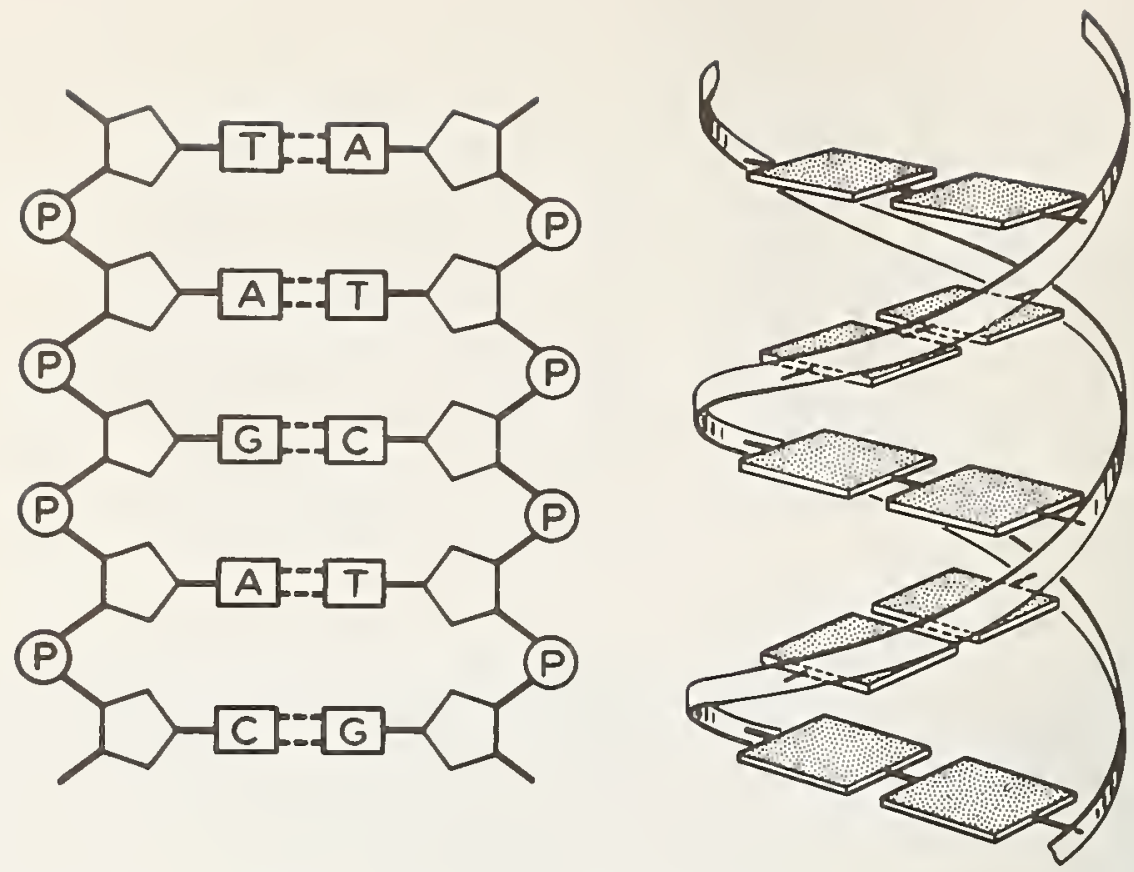

Figure 60. Schematic of double helix configuration of polynucleotide DNA.

\section{Conclusions}

We have sought to express the science and technology of solids at a time about midway in, or at least well beyond the earliest years of, the present solid state era. This means that certain factors have achieved strong growth in the last few decades, particularly the literature and bibliographic resources in the field. There has been special value during the adolescence of materials science and engineering in creating a common language, so that sophisticated bibliographic techniques could be introduced. This was done, for instance, in the Intrex program at MIT and has been extended by various universities and professional societies. Similarly, the theory of solids has been an exceptional stimulus, since earlier experimental methods were derived from centuries of classical mechanics, physics and chemistry.

In other words, we have sought to characterize typical qualities of a great multidisciplinary movement, which has benefited from generations of disciplined learning and discovery in the component fields. Such publications as the Annual Review of Materials Science have in the past few years produced a readily accessible survey of theory and experiment, which enables both the casual practitioner and deep scholar to master much of the new knowledge of materials. These records, as well as personal and institutional involvement, suggest that the future will be lively 
and appealing. Part of this comes from the inevitable stimulus of real world use of matter, part from the challenges and demands that economical and socially responsible usage in the future will impose. We have been unable in this short time to give proper attention to intellectual adventures of modern materials research and development and especially to the extraordinary theoretical progress of these current years. 


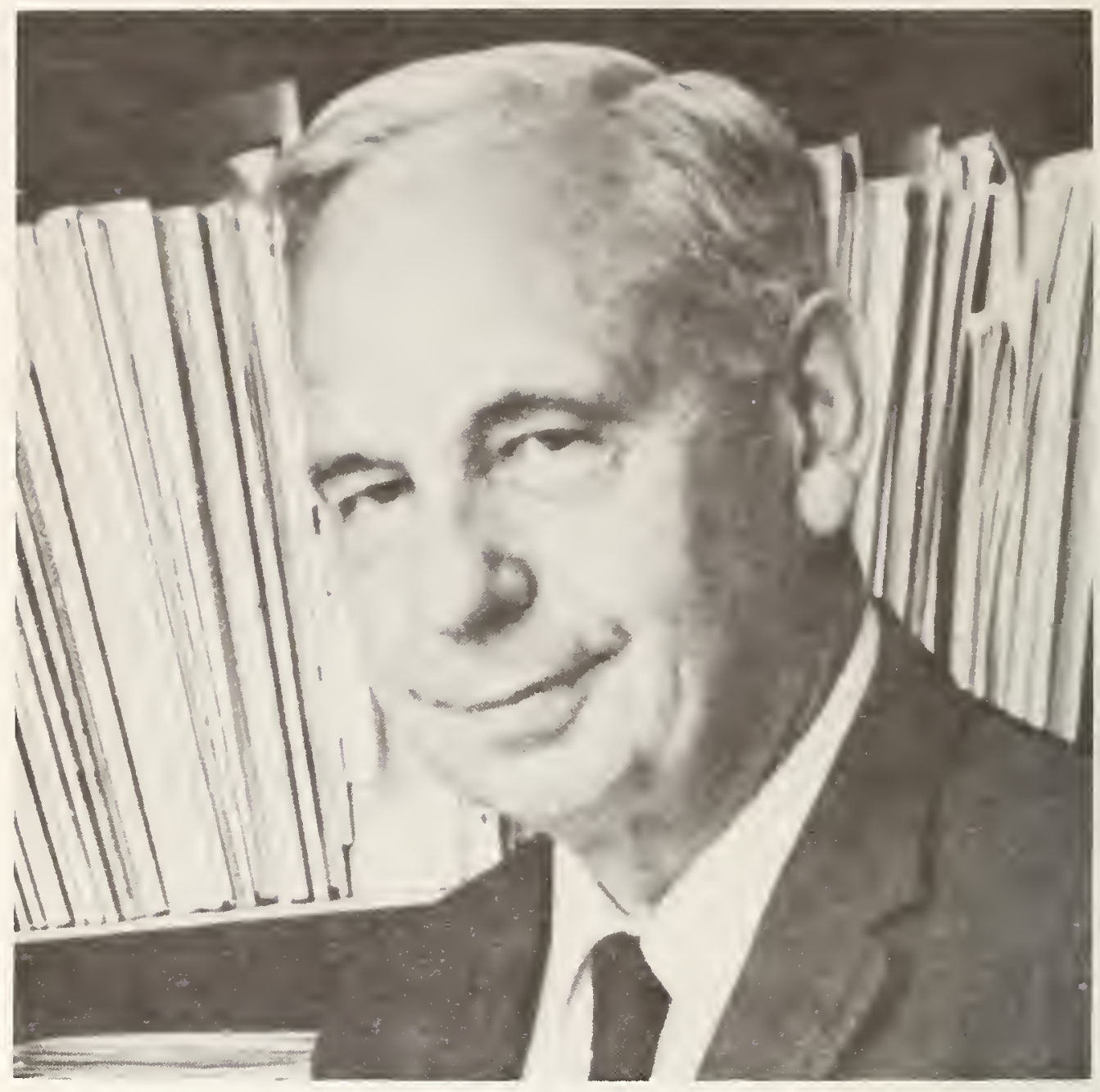

Professor Henry Eyring is the Distinguished Professor of Chemistry and Metallurgy at the University of Utah. He is widely known for his work in chemical reaction rate theory. His early work in this field has served as the foundation for its modern development. He has also contributed in major ways to the theory of liquids, molecular biology, and optical rotation. $\mathrm{He}$ is a member of the National Academy of Sciences and the Deutsche Academie der Naturforscher. Professor Eyring is also a former member of the National Science Board, former president and member of the Board of Directors of the American Association for the Advancement of Science, and a former president of the American Chemical Society. He has received the Joseph Priestly Medal of the American Chemical Society, the National Medal of Science, the Irving Langmuir Award, the Willard Gibbs Award, Peter Debye Award, and other honors. 


\section{CHEMISTRY - A CHANGING FRONTIER}

Being the same age as the National Bureau of Standards is an honor which will soon belong almost exclusively to the Bureau. In any case it gives me an interesting perspective.

The early years of man and institutions are usually devoted to finding their place in the grand scheme of things. My sensitivity to the chemical world became acute upon changing from mining and metallurgy and arriving at Berkeley in the fall of 1925 in quest of a Ph.D. in chemistry. At that time G. N. Lewis had noted that most stable molecules had an even number of electrons, also that bonds resulted in electron pairing and that many atoms tended to imitate the rare gases by surrounding themselves with eight electrons. However, the nature of the chemical bond and its strength was still the deepest of mysteries. The Periodic Table was powerful in systematizing chemistry as it still is, but the Bohr theory of the atom with the old quantum theory provided, at best, only a partial explanation of the Periodic Table.

Revolutionary changes, however, were in the wind. Heisenberg's matrix formulation of quantum mechanics was quickly followed by DeBroglie's explanation of quantization as the resonance of a wave associated with particles in periodic motion. Thus for the hydrogen atom one could write

$$
n \lambda=2 \pi r
$$

where $n, \lambda$, and $r$ are an integer, a wave length and the radius of the electron orbit respectively. This equation coupled with Bohr's quantization equation

$$
n h=m v r 2 \pi
$$

gives the equation

$$
h / m v=\lambda
$$

which was quickly verified by Davisson and Germer and by George Thomson. From this beginning Schrodinger developed wave mechanics much as we know it today. DeBroglie noted that for particles as for photons one could write for the energy of a particle $m c^{2}=h \nu$. Then, replacing the velocity $c$ by $\lambda \nu$ we obtain his equation $m c \lambda=h$. Here $m c$ is the momentum, where $c$ is the wave velocity which for a particle we write as $v$. Since the kinetic energy, $T$, has the value

$$
T=(m v)^{2} / 2 m=h^{2} / 2 m \lambda^{2}
$$


we see that the kinetic energy of particles is inversely proportional to the square of their wave length, $\lambda^{2}$. Thus when two atoms, such as hydrogen, come close together some fraction of the electrons' paths will encircle both atoms with a corresponding increase in wavelength and decrease in kinetic energy. It is accordingly not surprising that the principal contribution to the bonding energy is proportional to the number of such lengthened paths which encircle both nuclei-the so-called exchange bonding. One can conveniently think of the decrease of momentum with wave length as claustrophobia which is lessened by an electron lengthening its path by encircling more than one nucleus.

Pauli's interpretation of spectra whicu sed him to state that two electrons occupying the same orbital must have opposite spins also explained G. N. Lewis' rule that two electrons pair to make a chemical bond.

The activation energy of reaction rates also became understandable as the work of partial promotion of electrons into upper states arising from the electronic reorganization occurring in the activated complex as the system passes through the change into a new way of bonding. The activated complex is that point along the pass for which energy-wise it is downhill to reach either reactants or products, i.e., a point of no return on the energy hypersurface. Low-lying troughs or valleys on such energy hypersurfaces correspond to compound formation. Quantum and statistical mechanics together with the use of the theory of small vibrations enables us to calculate all the properties of stable or activated states of chemistry.

The excitement arising from the prospect of pure ab initio chemistry, coming as it did before we had computers, faded rapidly. With digital com puters prospects have become much brighter, but many problems are still best left to the analog computer, i.e., the chemical laboratory.

More important than the numerical results so far coming out of most ab initio calculations are the frames of reference quantum mechanics gives for systematizing all of chemistry. The use of the energy hypersurface has clarified our understanding of chemical reactions of all kinds. Raising molecules to upper energy hypersurfaces as in mass spectrography, photochemistry or through using other types of radiation leads to transitions back to the lower surface. Such transitions may be radiationless at the point where hypersurfaces nearly cross while at other points such transitions involve radiative processes. Hund and Mulliken's correlation diagrams for diatomic molecules constructed using symmetry relations indicate how a state for the separate atoms passes into a particular state for the united atom. Thus symmetry of the system is the guiding principle. No crossing of lines on the diagram occurs for states of the same symmetry but may occur for levels with different symmetries. Woodward and Hoffman have drawn analogous correlation diagrams for energy levels joining reactants and products for many types of reactions. This procedure has led to successful predictions for photochemical and thermal reactions, and natu- 
ral extensions will aid in systematizing future studies in this field. This brings up the question of how reaction rate data should be reported.

If a reaction rate has been well reported, the procedure for tabulation is straightforward. The authors' rate equations should be specified with the constants they have established. This will make it possible for other investigators to use the data in testing theories without loss of pertinent information. An ever-present question is whether the mechanism has been correctly established. If the work is borderline, a reference to it in the tables should suffice. When elementary reactions have been sorted out and values for the rates have been established over any extended range of temperatures, pressures, solvents or phases, absolute reaction rate theory provides a powerful tool for extrapolation and of relating the observed results of elementary reactions to molecular structure. This use of the data is not infrequently hampered by misconceptions. A brief review of the theory of reaction rates should be helpful.

The rate of any reaction, $R$, involving a region of almost no return on the energy hypersurface, takes the form

$$
R=\frac{\kappa}{2} C_{\delta}^{\ddagger} \frac{\overline{\dot{\chi}}}{\delta}=\frac{\kappa}{2} C^{\ddagger}\left(2 \pi m^{\ddagger} k T\right)^{1 / 2} \frac{\delta}{h} \sqrt{\frac{2 k T}{\pi m^{\ddagger}}} / \delta=\kappa \cdot \frac{k T}{h} C^{\ddagger}
$$

Here $\delta, C_{\delta}^{\ddagger}, C^{\ddagger}, \chi, \kappa$ have the following meanings, respectively: a short distance normal to the barrier (i.e., along the reaction coordinate) in the region of no return, the concentration of activated complexes in a length $\delta$ along the reaction coordinate, the concentration of these complexes in the lowest level in the degree of freedom along the reaction coordinate, the velocity along the reaction coordinate, and finally the transmission coefficient which corrects for repeated barrier crossing and is frequently very near unity. Contrary to what is frequently supposed, using the rate of reac. tion at points away from equilibrium as we have done in the above deriva. tion does not imply anything special about equilibration between the trajectories approaching the point of no return. Rather it rests on the fact that deletion of the product molecules responsible for the back reaction is without significant effect on the forward reaction measured at the point of no return because there is no intercommunication and therefore no perturbation of the forward rate by deleting the back reaction. $\kappa$ corrects for multiple barrier crossings and quantum mechanical reflection. Barrier leakage should of course be added to the rate. Remembering the thermodynamic relations $\left.C^{i}=F_{i} \gamma_{i}, \gamma_{i}=e^{\mu_{i}} / k T, \mu_{i}=\partial A_{i} / \partial n_{i}, A_{i}=-k T \ln \Sigma_{i} \exp -\epsilon_{i} / k T\right) \Delta G_{x}^{\ddagger}$ $=-k T \ln K^{\ddagger}=-k T \ln C_{i}^{\ddagger} \gamma_{i}^{\ddagger} / \Pi_{i}^{\ddagger} C_{i}^{\ddagger} \gamma_{i}$, it should be clear that eq (5) is applicable to elementary reactions in all phases and conditions involving points of no return, or nearly no return. The symbols used have their usual meaning and so need not be defined further. 
Absolute Rate theory in reducing kinetics to the language and concepts of thermodynamics achieves an intuitive clarity that is too often incompletely utilized. Very much is to be gained in predictive effectiveness, by thoughtful interpretation of good data yielding entropies, heats and volumes of activation for elementary processes. This procedure will un. doubtedly bring about great future systematization of reaction kinetics so that many fewer experiments will be necessary with the accompanying economies.

Reaction rate studies in which reactants are restricted to a few specified quantum states are yielding products in correspondingly less numerous final states. The use of tuned lasers in the separation of isotopes is a case in point. If either fission or fusion are ever to become the main economic sources of power, more efficient separation of isotopes will be one of the important obstacles to be overcome. The recycling of materials involves overcoming even more formidable obstacles and merits our best efforts. Recycle or perish seems to be the dilemma that confronts us as we exhaust our natural resources. We will succeed in meeting these difficult challenges because we have to, but the way will be greatly simplified by a lot of advance planning.

A shortage of chromium, for example, influences our African policy. More critical are the compromises which will confront us if we run short of fuel ahead of powerful antagonists. Our most pressing problems are not only the development of wise national policies with respect to these shortages that do and will confront us, but even more difficult for us, that the public must be educated to accept and implement the programs devised to alleviate the difficulties. In our democracy even the President is powerless if he lacks popular support.

Because our national policymakers are less than omniscient, all scientists share the responsibility of supplying them with the best technical information available. The National Bureau of Standards because of its influential position thus shares a dual responsibility in this connection: first, of supplying the best possible information to the policymakers and then, of doing all it can to make the resulting policies acceptable to an enlightened public.

The long delay in the wide adoption of the metric system in our indus try is an example of the reluctance of private business to absorb the heavy expense of a quick change even though this might be less expensive overall. This same reluctance of private enterprise to absorb unacceptable losses prevents industry from going all out in providing us gasoline from coal. A segment of industry must be subsidized by the government to produce, without loss, needed materials against the day when the present cheaper sources dry up. Any other procedure seems to invite disaster. How can the Bureau of Standards help map out procedures which will usher us into a new era in which we have switched our consumption of materials to 
sources that are inexhaustible, because they are being continually renewed? This will not be easy but must come eventually. We cannot solve all our problems at once, but a sensible ordering of priorities among the many problems claiming our attention should be developed. Any intelligent planning seems better than no effort to meet these situations that if neglected could be disastrous.

The bright side of this picture is the technical competence we have either to make the materials we need from available sources or to find acceptable if not ideal substitutes. Illustrating this point, many striking examples of human inventiveness precipitated by necessity could be cited. We will mention only a few. At the beginning of this century fixed nitrogen for fertilizers and explosives was obtained from Chile's deposits of saltpeter. Germany was thus at the mercy of the British fleet which controlled the seas until Fritz Haber and other German chemists perfected the Haber process for fixing nitrogen in the form of ammonia by using an iron catalyst. This discovery made the disastrous first World War possible. Besides illustrating human creative ability, this example illustrates the fallibility of human judgment in making use of new discoveries and inventions. Another illustration of wartime inventiveness was the Germans' use of gasoline from coal to keep their war machine running when they were cut off from the usual sources. In World War II when the Japanese cut off the supply of natural rubber, American chemists within little more than a year were producing streams of artificial rubber which grew into a torrent. For many uses the synthetic rubber was better than the natural product. Even more revolutionary was the development of the atomic bomb during World War II with the hydrogen bomb following soon after. Space exploration is a child of the electronic revolution, but it, too, had its remarkable chemical components in the fuels and the materials developed to withstand the extraordinary demands for lightness, strength, severe changes in temperature and freedom from corrosion.

Wind, sunlight, heat from the earth, fission, and perhaps fusion, if the hydrogen bomb can be tamed economically, together with recycling materials in short supply and more economical use of power in general: such sources should be phased into general use as fossil fuels phase out. This change could occur without a cataclysmic disaster if man faces up to what lies ahead and has the moral fortitude to sacrifice while there are still options that can be implemented with hard work and wise planning. If we cannot do this, mankind is going to pay with unspeakable hardships for his monumental folly. The hour is certainly very late, but not too late to avoid complete disaster if we set about developing the options still open to us, now. That the human race will survive seems probable and there will be discoveries and inventions not dreamed of, born of the painful struggle for survival, as there have always been in the past. 


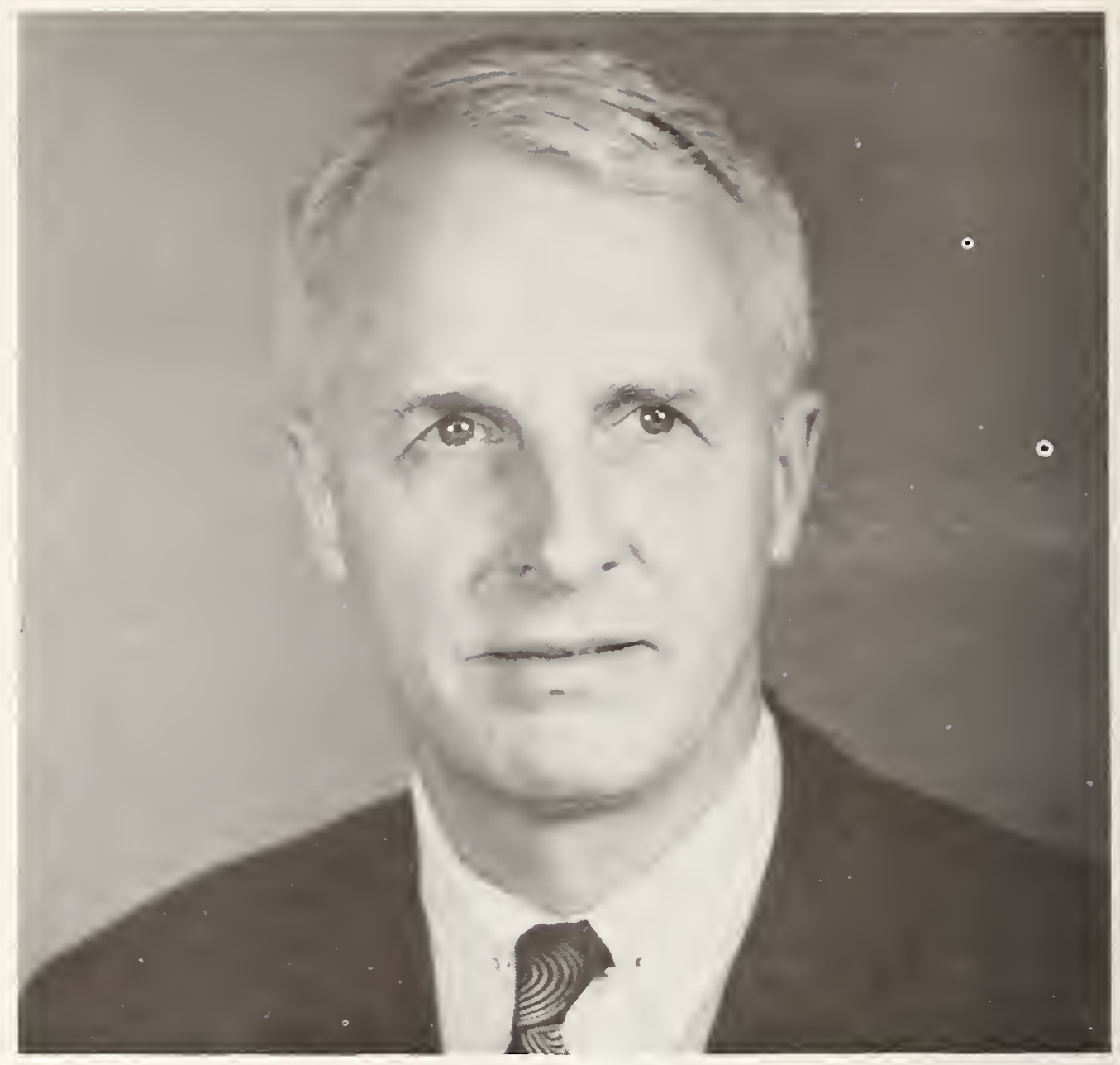

Professor Garrett Birkhoff is the Putnam Professor of Pure and Applied Mathematics at Harvard University. He is noted for his investigations in modern algebra, fluid mechanics, numerical analysis, and nuclear reactor theory. He is a member of the National Academy of Sciences and Vice President of the Mathematical Association of America. Formerly, he was President of the Society for Industrial and Applied Mathematics, Vice President of the American Mathematical Society and the American Academy of Arts and Sciences, and chairman of the Conference Board of the Mathematical Sciences. He was a Walker-Ames Lecturer at the University of Washington and Taft Lecturer at the University of Cincinnati. 


\section{APPLIED MATHEMATICS AND ITS FUTURE}

\section{Introduction}

Essentially, mathematics becomes "applied" when it is used to solve real-world problems "neither seeking nor avoiding mathematical difficulties" (Rayleigh). Thus applied mathematics ecompasses the interdisciplinary aspects of mathematics.

The preceding definition is very loose, and I shall not dwell on it. All branches of mathematics are potentially applicable, and indeed as soon as one important application is found for a particular mathematical idea or technique, analogy will often suggest others. For this reason, the nature of applied mathematics is continually changing.

Moreover the best applied mathematics is often done by persons who are not professional mathematicians of any kind, let alone applied mathematicians. Correlated with this is the fact that the most important areas of application of mathematics, such as mathematical physics, mathematical statistics, and the mathematics of computing, tend to split off from the rest of mathematics, and to become new specialties with their own professional societies.

This tendency makes the content of applied mathematics not only time-dependent but even unstable: because of its inherent close relation to other subjects, pieces of it are constantly being captured by experts in these subjects and taken away from mathematicians.

This instability is only one of many difficulties that beset the professional applied mathematician. Another is the fact that mathematical formulations of real-world problems are apt to be impossibly complicated or ridiculously simple. In the first case, they are intractable; in the second, their solution does not require the services of a professional. In the broad spectrum of real-world problems, very few fit neatly into that narrow complexity band of those that can be solved by a skillful applied mathematician in from 1 to 5 years. As a result, the most important branches of applied mathematics in real life are still arithmetic and elementary logic!

No attempt to present a comprehensive view of applied mathemaucs and its future can hope to escape from the uncertainties and ambiguities implied by the preceding observations. However, I shall do my best, and I hope my talk will occupy an honorable place beside the 1900 review by $R$. S. Woodward [29] of 19th century progress in applied mathematics, and the description [6] by Thorton Fry of the state of American industrial mathematics in 1941. 


\section{Ptolemaic Geometry and Astronomy}

The past usually provides the best guide to the future, and I shall begin by discussing briefly the oldest applications of mathematics: to geometry and astronomy.

The basic facts of geometry and astronomy were known to prehistoric man, and certainly the Babylonian high priests were experts on both. But it was Euclid who first made geometry into a branch of mathematics, by deducing it all from a relatively small number of geometric postulates and axioms about "quantities" in general. His deductive exposition, written in Alexandria under the Ptolemies, has stood the test of time very well!

Ptolemaic astronomy also provided a mathematical model which predicted very successfully the regular gyrations of the sun, the stars, the moon, and the planets as seen from our earth. In essence it seems to have been a kind of data fitting: finding the phase constants $T_{n}$, frequencies $k_{n} / 2 \pi$, and amplitudes $a_{n}$ that gave the best fit to almost periodic empirical functions:

$$
u(t) \simeq \sum a_{n} \cos k_{n}\left(T-T_{n}\right)
$$

Copernican astronomy gave no better agreement with observations.

To set the tone for the rest of my talk, I want to emphasize that these marvelous achievements of the human mind were not the result of unaided thought, but that carefully designed instruments for measuring distances and angles dating from prehistoric times, and observations extending over centuries were required for their scientific verification.

Also, some of the simplest questions could not be answered by applying known mathematical ideas. Thus Pythagoras was already confronted by a serious dilemma: he knew that the ratio $\sqrt{ } 2$ of the hypotenuse to the side of an isosceles right triangle could not be expressed as a ratio of integers ("rational number"), and swore his followers to secrecy on the subject. This led to the discovery of irrational numbers. Again, the Greeks could find no way to trisect a general angle, "square a circle," or duplicate a cube with ruler and compass. Much later, it was shown why this could not be done: ${ }^{1} \pi$ is a transcendental number, and the Galois group of ${ }^{3} \sqrt{ } 2$ is not of order $2^{n}$.

However, there were simple enough practical ways to approximate $\sqrt{ } 2$ to any desired accuracy by a rational fraction, and to approximately trisect any angle or duplicate any cube likewise. This brings out a crucial point, to which I shall recur: unsolved scientific problems need not impede engineering progress.

The next great advance in geometry came with Descartes and Fermat around 1637. It consisted in using rectangular coordinates to represent

${ }^{1}$ Felix Klein, "Elementary Mathematics from an Advanced Standpoint." 
curves. This powerful application of the then new technique of algebra (symbol-manipulation) to solve geometric problems revolutionized geometry, and made it possible to go far beyond Appolonius, Pappus, and other Ptolemaic geometers.

\section{Celestial Mechanics}

Probably the most impressive early applications of mathematics were to celestial mechanics. We all know that Kepler spent years using the best available instruments to help him interpret the observed motions of the planets in the sky in terms of the Copernican heliocentric hypothesis. He finally succeeded in summarizing his observations and calculations in three laws:2

(i) The planetary orbits are ellipses, with the sun at one focus.

(ii) The area is swept out by a radius vector from the sun to any one planet at a constant rate,

(iii) The periods of the planets are proportional to the three-halves power of their median distances.

The mathematical skill required to make Kepler's discoveries is less well publicized. Besides using logarithms (a new invention at the time) to facilitate his calculations, he had to know the properties of the conic sections and spherical trigonometry. Just to convert his measurements from a geocentric to a heliocentric coordinate system must have been a major task. Although the "fixed" stars made it relatively easy to know the direction of any planet (or moon or the sun) from the observing telescope, to locate such a heavenly body precisely in four-dimensional space-time required ingenuity, patience, and very accurate clocks. ${ }^{3}$

Everyone also knows Newton's remarkable achievement: by developing and applying the ideas of the calculus adumbrated earlier by Cavalieri, Fermat, Pascal, and Barrow, ${ }^{4}$ he was able to deduce Kepler's three laws from a single hypothesis: that planets were attracted towards the sun by a "gravitational" force per unit mass, inversely proportional to the square of their distance from it. Thus, by accepting the law of gravitation as a universal principle, applicable to all bodies, he was able to "account for all the motions of the celestial bodies, and of our sea."'5

${ }^{2} J$. Kepler, Harmonica Mundi (1619).

${ }^{3} \mathrm{~A}$ good reference is W. Chauvenet, Manual of Spherical and Practical Astronomy, 2 vols. (Lippincott, Philadelphia, 1855: Dover, 1960).

${ }^{4}$ See D. J. Struik, Source Book in Mathematics: 1200-1800, chap. IV (Harvard Univ. Press, 1969).

${ }^{5}$ Harlow Shapley and Helen Howarth, Source Book in Astronomy (Harvard University Press, 1929), p. 78. This book also contains relevant excerpts from the work of Kepler. 
Newton's Philosophiae Naturalis Principia Mathematica (first ed., 1687) contains of course very much more than this, including the result that any spherically symmetric body exerts the same gravitational attraction as if its mass were concentrated at its center. Another conclusion, deduced more rigorously in 1742-3 by Maclaurin and Clairaut from the hypothesis that the earth's shape conforms to equilibrium between the centripetal force of gravitation and the centrifugal force of rotation, is that the shape of the earth is an oblate spheroid, contrary to the generally accepted contemporary view that it is prolate. Maupertuis was to verify the correctness of Newton's and Clairaut's conclncions a century later (see sec. on Continuum Physics).

Newton's dramatic success stimulated many attempts to extend his results. The most comprehensive extension consists in Laplace's five volume Mécanique Céleste (1799-1825), translated into English by Nathaniel Bowditch 10 years later (1829-39); it includes careful discussions of tidal phenomena. Laplace claimed to have proved the stability of the solar system: that no planet would ultimately leave it as a result of the accumulated perturbations of its orbit around the sun by all the other planets. However, his "proof" was not rigorous, to say the least. ${ }^{6}$

I mention this to emphasize the point I have already made: that the systematic application of known techniques often fails to answer very simple and natural questions. Celestial mechanics provides other examples. The three body problem, first attacked by Lagrange in 1772 (Shapley, ${ }^{5}$ pp. 131-2), still defies solution. It is not even known whether it possesses any invariants ("integrals") besides those implied in the classic laws of conservation of energy, (linear) momentum and angular momentum.

To be sure, it has had many triumphs, including the prediction of the existence of new planets (Ceres, Neptune, Pluto), and the ability to predict eclipses and reconstruct past eclipses of the sun and moon for millenia. But one of the most fundamental scientific discoveries to which it has led, through the meticulous comparison with observation of the consequences of Newton's theory of the solar system, is that of deviations from the inverse square law of universal gravitational attraction. The observed small but systematic advance in the perihelion of Mercury, unpredicted by Newton. was one of the three "crucial effects" that led Einstein to postulate his theory of general relativity in our century. ${ }^{7}$

${ }^{6}$ See H. Poincaré, "Sur la Stabilité du Système Solaire," (Annuaire du Bureau des Longitudes, 1898); [29, p. 52].

'Presumably, the failure of Einstein's earlier theory of special relativity to predict it was another! 


\section{The Exponential Function}

So far, I have just recalled how the calculus made possible the development of celestial mechanics, the oldest branch of mathematical physics after geometry, into a subject of great mathematical depth and precision. I now wish to recall an entirely different aspect of applied mathematics, enormously relevant to biology and economics: the related concepts of the exponential function $e^{z}$ and the growth rate definable empirically as the logarithmic derivative $d x / x d t$.

It was of course Euler who discovered the marvellous connection in the complex domain between the exponertial function and the trigonometric functions, exemplified by the classic turmulas.

$$
\cos x=\left(e^{i x}+e^{-i x}\right) / 2, \sin x=\left(e^{i x}-e^{-i x}\right) 2 i, e^{\pi i}=-1 .
$$

At the time of their discovery, these formulas must have seemed to constitute abstract pure mathematics of the most esoteric kind, yet the fruitfulness of their discovery for applications is incalculable.

Instead of dwelling on this well-known fact, however, I want to make a much more simple and down-to-earth application of this same function in the real (and real world!) domain, to the supply of people in the United States trained to do mathematical research. There were very few such persons in 1876, perhaps 15 would be a fair estimate. Today, we have about 15,000 mathematical Ph.D's, and a quick examination of the available data suggests that the growth rate in the intervening decades has been about 7 percent annually.

This conforms to the formula $x=x_{0} e^{0,07 t}$, doubling every 10 years as our consumption of electrical power has. The growth factor is thus 8 every 30 years (roughly a generation), a thousand every century, a million every 200 years, and would be a million trillion trillion if it could be maintained for a millenium.

Many phenomena that we take for granted are simple mathematical corollaries of this growth rate. With a 7 percent annual growth rate, half of all we know will have been discovered in the last decade! With a 3.5 percent annual growth rate, perhaps more typical of the mathematical world as a whole, it will have been discovered in the last 20 years. This makes the study of the history of mathematics or even of any mathematics more than 40 years old, seem to have little value. After all, it can refer to only onefourth of what we know today (and generally the stalest part) if the growth rate is 3.5 percent; with a 7 percent annual growth rate, the fraction would be one-sixteenth! In other more rapidly developing fields, presumably having greater research potential, the fraction is much, much less.

Another corollary refers to that unfelicitous phrase: "having" graduate students. With a 7 percent growth rate, were there no mathematical immigration except by degree-seeking graduate students, the average Ph.D. 
can expect to have "had" (or "produced") about four graduate students during a period of maximum "Ph.D. productivity" between his 10th and 25 th post-doctoral year. If only one-fourth of all Ph.D's try to reproduce their kind, the number grows from 4 to 16 . If more maturity and experience are asked of $\mathrm{Ph} . \mathrm{D}$. Thesis supervisors, so that such supervision normally takes place from 15 to 35 years after receiving the doctorate, the number grows to 30.

These numbers give a roughly correct statistical picture of what has been going on over the past century; it ignores of course some very substantial mathematical immigration. Were it to continue for another century, we would have 15 million Ph.D. mathematicians by 2076, publishing (if successful in fulfilling their mission) at least 100 million pages of new mathematics annually! This seems unlikely!

On the other hand, consider the dismal picture of a zero Ph.D. population growth rate. Obviously, the average Ph.D. can hope to "have" only one Ph.D. during his lifetime. Even if three-quarters of our Ph.D.'s are sterile, the remaining fertile ones can only hope to "produce" one every 5 years for 20 years, or one every 7 years for 28 years.

I do not wish to push this elementary application of well-known mathematics any further: it is too painful! But I hope you will have the properties of a 7 percent growth rate in mind later on, when I take up mathematical economics. And I hope that any pure mathematicians who consider it will realize how much better the prospects of academic mathematicians for supervising graduate students would become, if 60 percent of all Ph.D.'s could get nonacademic jobs!

\section{Hydraulics and Hydrodynamics}

I shall now return to applications of mathematics to physical phenomena: "natural philosophy" in the classical sense. Hydraulics has existed as an engineering science since early antiquity; ${ }^{8}$ the Egyptian irrigation system and Roman aqueducts provide monuments to it.

In his Principia Newton already proposed an ingenious mathematical model for air resistance, but it assumes that air consists of stationary molecules, and provides a decent approximation only for hypersonic missiles in a near vacuum, which is certainly not the application he had in mind.

It was Daniel Bernoulli and Euler who first constructed a plausible model for the behavior of fluids, thus founding continuum mechanics in the modern sense. They postulated an (ideal) incompressible nonviscous fluid; it can be successfully applied to explain many facts about waves and tides.

${ }^{8}$ See H. Rouse and S. Ince, History of Hydraulics (Iowa Inst. of Hydraulic Research, 1957). 
It has the remarkable property that motions of such a fluid started from rest always admit a velocity potential $\phi$ satisfying the Laplace equation $\nabla^{2} \phi=0$.

Lagrange believed that the idealized fluid of Bernoulli and Euler would reduce fluid mechanics to a mathematical science, like geometry and celestial mechanics. He wrote in his classic Mécanique Analytique (1788):

"One owes to Euler the first general formulas for fluids motion... presented in the simple and luminous notation of partial differences... By this discovery, all fluid mechanics was reduced to a single point of analysis, and if the equations involved were integrable, one could determine completely, in all cases, the motion of a fluid moved by any forces..."

However, he was under an illusion. Actually, Stokes was to succeed around 1840 in integrating these equations, and calculating the pressure distribution around a sphere as predicted by the model. He found that, as d'Alembert had guessed in 1750 , the pressure had fore-and-aft symmetry, so that the net force was zero. This was contrary to the well-known empirical fact (also predicted by Newton's model) that solids moving through fluids of density $\rho$ encounter a resistance $F$ roughly proportional to $\rho v^{2} A$, where $A$ is the cross-section area and $v$ the velocity. More precisely, we have $F=1 / 2 \rho C_{D} v^{2} A$, where the empirical "drag coefficient" $C_{D}$ as defined by this equation from the measured $F$ ordinarily lies between 0.1 and 2. Lagrange did not mention this "d'Alembert paradox" in his book, and his example has been followed by most subsequent writers of books on fluid mechanics.

The most notable of these is Lamb's Hydrodynamics (first ed. 1879, sixth ed. 1932), of which the best half is devoted to potential flows. These can be constructed by the methods of potential theory, which also applies to gravitational fields and to electrostatic and magnetostatic fields, as well as to functions of a complex variable. This fact made potential theory a central topic in mathematical physics in the 19th century.

Nearly a hundred years after Euler, Navier (1821) and Stokes (1845) developed an alternative model for liquids and gases: that of an incompressible viscous fluid. Unfortunately, their equations are nonlinear and so complicated that they have been integrated analytically in only a very few cases to this day. The same can be said of the model of a compressible nonviscous fluid. Not long after, Helmholtz and Kirchhoff showed that fluid resistance could be qualitatively explained within the framework of potential theory, by admitting the possibility of flow separation and wake formation.

In 1904, Prandtl reconciled the ideas of Helmholtz and Kirchhoff with the Navier-Stokes equations, by postulating a composite model, according to which solids moving through fluids (liquids or gases) were sheathed in a thin boundary layer, inside which the flow is nearly parallel to the solid surface, and the Navier-Stokes equations apply in a very simplified form. Outside of this boundary layer he assumed potential flow. 
Unfortunately, under many practical conditions the flow in the boundary layer is not "laminar" (i.e., time-independent) as was assumed by Prandtl, but turbulent. In particular, the flow is turbulent in most problems of hydraulics, such as arise in supplying water or hydroelectric power to cities. It is also the case in aeronautics, both at low and intermediate flight speeds; for aircraft flying at high subsonic and supersonic speeds, the modeling of real flows is further complicated by compressibility affects.

Faced with these complications, engineers of many kinds (hydraulic, civil, mechanical, aeronautical and chenical) use all the models I have mentioned, but selectively, on the basis of engineering experience and judgement. To apply mathematical ingenuity freely to plausible and interesting assumptions, in the same way that most pure mathematicians do, would have led to a long series of engineering disasters and neglected opportunities. I shall next try to explain this general principle more concretely.

\section{Engineering Models}

Engineers tend not to worry too much about the philosophical consistency of the principles underlying their mathematical models, provided that these models agree reasonably well with observation over the range of interest. If a model simulates reality well enough, for whatever reason, it is a cheap substitute for possibly destructive tests of large families of artifacts in design studies. The model can include empirical functions ("correlations," "constitutive equations," etc.) quite freely, provided that they conform to engineering experience and judgement. For this reason, engineers will always make extensive use of applied mathematics.

Scale Models. However, models need not be mathematical: one can build geometrically similar models to scale, and use the concept of proportion to scale the resulting observations up or down. If the hydrodynamical differential equations of Euler and Lagrange were exact, it would be easy to do this: thus the dimensionless "drag coefficient" $C_{D}=2 D / \rho v^{2} A$ would depend only on shape, and not on the fluid or the size and speed of the moving object.

Even if these equations were exact, ship wave resistance would depend on the Froude number $F=v / \mathrm{Vgl}$ ("speed-length ratio"), because of the presence of a free surface. However, it would otherwise (scaled as above) depend only on the hull form, and so one could test for this using small, relatively cheap, scaled down models.

If one admits that compressibility is important, but neglects viscosity, then the drag coefficient should depend only on the shape and Mach number $M=v / c$ : the ratio between the missile speed and sound speed. This is usually a good approximation in ballistics. 
Finally, if viscosity $\mu$ is important but $v<<c(M<0.2$, say), then the resistance coefficient would depend only on the shape and the Reynolds number $R=\rho d v / \mu$. This law of "similarity" applies also to turbulence effects, as was verified experimentally by Stanton and Pannell in 1920: they showed that even air and water were "similar" in this technical sense: at the same Reynolds number, turbulence in water and turbulence in air are exactly similar in all respects. ${ }^{9}$

Because it was so difficult to integrate the Navier-Stokes equations analytically in any but the simplest cases (and impossible to do so in the presence of turbulence), airplane design was based almost entirely on wind-tunnel tests during the crucial period 1910-1950, and the role of theoretical fluid mechanics was limited to interpreting experimental data.

On the other hand, geometry (the oldest branch of applied mathematics) was basic to fitting together the needed components, while Lagrange's classical theory of small oscillations was effectively applied to minimize vibration and flutter.

This illustrates an important point: for applied mathematics to help engineers, mathematical models must be economically competitive with other approaches such as the use of scale models. In addition, they must be reliable within the contemplated range of operating conditions.

Contrast with Mathematical Physics. The preceding comments are intended to bring out the point that engineers and physics create and adopt mathematical models for very different purposes. Physicists are looking for universal laws (of "natural philosophy"), and want their models to be exact, universally valid, and philosophically consistent. Engineers, whose complex artifacts are usually designed for a limited range of operating conditions, are satisfied if their models are reasonably realistic under those conditions. On the other hand, since their artifacts do not operate in sterilized laboratories, they must be "robust" with respect to changes in many variables.

This tends to make engineering models somewhat fuzzy yet kaleidoscopic. In fluid mechanics, Prandtl's "mixing length" theory and von Karman's theory of "vortex streets" are good examples; the "jet streams" and "fronts" of meteorologists are others.

\section{Molecular Models}

Newton's success in deducing the observed planetary orbits from an assumed inverse square law of gravitation inspired other particle models on a microscopic scale. These models attempted to deduce the behavior of solids, liquids and gases from analogous assumptions.

${ }^{9}$ For the mathematical theory of scaling, see G. Birkhoff, Hydrodynamics: A Study in Logic, Fact and Similitude, 2d ed. (Princeton University Press, 1960). 
Thus D. Bernoulli showed in his Hydrodynamics (1738) that the pressure of a gas on the walls of a container could be explained qualitatively as a reaction to smooth spherical molecules of finite radius bouncing off them. In 1743 , Boscovich suggested trying to explain elasticity by assuming that any two "ultimate particles" attract or repel each other along the line joining them according to some law of force $F=f(r) .{ }^{10}$ This idea was used by Poisson in 1812 to construct a short-lived theory of plates; in 1819, Laplace constructed an analogous but more plausible molecular theory of surface tension (capillary forces).

In 1821, Navier applied similar ideas even more successfully, to derive "constitutive equations" for a homogeneous isotropic elastic solid. These equations contained a single constant $C$, the compressibility, and implies that the "Poisson ratio" of lateral contraction to longitudinal extension in pure tension must be $1 / 4$. This "uniconstant" model was discarded later in favor of a "variconstant" continuum model proposed by Green (1837) and Stokes (1845). They showed that isotropic linear dependence of a general stress tensor on a strain tensor involved two arbitrary constants, the shear modulus and the bulk modulus. ${ }^{11}$ Experiment confirmed that these could vary independently, depending on the material - and that the Poisson ratio need not be $1 / 4$.

The ideas of Poisson and Green were extended to anisotropic solids (crystals) by Cauchy and Lamé, respectively, and still more sophisticated molecular models of solids were proposed later by Kelvin, Max Born, and L. Brillouin, who applied them to deduce specific heats of solids, piezoelectric effects, and propagation laws for electrical waves along transmission lines. ${ }^{12}$ However, such molecular models of solids have been increasingly displaced by more sophisticated quantum-mechanical models over the past 50 years, while elasticity theory ignores both!

Kinetic Theory of Gases. Much greater success has been achieved with molecular models for gases. By assuming that molecules are small groups of atoms connected by springs, the pressure and specific heat of nondense gases have been quite well explained. Moreover this model of "heat as a mode of motion" (Tyndall) bridges nicely the transition from mechanical to thermal energy; as one of many by-products, we get a plausible explanation from first principles of the adiabatic equation of state, $n=k \rho^{1.4}$, for diatomic molecules.

\footnotetext{
${ }^{10}$ For the facts stated here, see [2]], chap. V and VI; also Todhunter and K. Pearson, History of the Theory of Elasticity (Cambridge Univ. Press, 1886).

"Cauchy's first memoir (1822) had involved two constants, but his later papers only admitted one. And indeed, any simple periodic array of like static molecules, in equilibrium under mutual attraction and repulsion, must have a Poisson ratio of $1 / 4$.

${ }^{12}$ L. Brillouin, Wave Propagation in Periodic Structures (McGraw-Hill, 1946).
} 
I could not begin to describe all the intermolecular and interatomic "force laws" $F=f(r)$ that have been proposed, in the spirit of Boscovich and Laplace, to explain the observed facts-including the "triple state" of matter and the behavior of the equation of state near it. Although these models are all inconsistent with what we know about the electrical nature of interatomic forces and quantum mechanics, they constitute a fascinating application of mathematics to pure physics. In particular, they provide by far the best theoretical model for diffusion phenomena in gases, including (molecular) viscosity and thermal conductivity. However, in most engineering problems, it is simpler and more reliable to assume empirical values of viscosity $\mu$, thermal conductivity $\kappa$, and specific heats $C_{p}$ or $C_{t}$, than to worry $w h y$ they have the values that they do, or why the ratio $\kappa / \mu$ should take on a particular numerical value.

If one does this, it is usually also much easier to estimate the small effects of diffusion than the much larger effects of convection that I discussed previously in the section on Hydraulics and Hydrodynamics.

\section{Continuum Physics}

Mechanics is the oldest and most highly developed branch of physics, after geometry. Indeed, in the Encyclopadie der Mathematischen Wissenschaften [33], 3000 pages are devoted to mechanics, and only 2000 pages to all the rest of physics and physical chemistry. I shall briefly recall next a few of the basic ideas that mathematicized the other branches of physics, at least in principle.

We all know that Fourier showed in 1807-22 how one could treat general initial and boundary value problems for heat conduction in slabs, cylinders, and spheres by making suitable use of Fourier series and in. tegrals. His analytical methods were generalized and rigorized during the next 100 years by Dirichlet, Riemann, Poincaré, F. Riesz, Plancherel and others. During the same period, they were brilliantly applied to a wide range of other wave phenomena by Helmholtz, Kelvin, Rayleigh, etc. For example, they helped Helmholtz to design primitive speaking machines, simulating the human voice.

The development of a successful electromagnetic theory was much slower; the steps involved have been ably reviewed by E. T. Whittaker [34]. In 1857, Kirchhoff discovered that the velocity with which an electric disturbance is propagated along a perfectly conducting aerial wire is equal to the velocity of light $[34, \mathrm{pp} .232,254$, ]. This simple arithmetic observation was of capital importance, because it gave the first indication that light is an electromagnetic phenomenon.

Using assumed analogies with elasticity and particle dynamics to guide his model-building, Maxwell was able in 1864 to present to the Royal 
Society the architecture of his new mathematical theory of electromagnetism, "stripped of the scaffolding by aid of which it had first been erected" [34, p. 255]. Nine years later he published the first edition of his classic Treatise on Electricity and Magnetism, stating in its preface that "I shall avoid, as much as I can, those questions which, though they have elicited the skill of mathematicians, have not enlarged our knowledge of science."

About 50 years later, and 50 years ago, Schrödinger constructed his famous partial differential equations for atomic physics. Like Maxwell, he was guided by mechanical analogy. Although his equations are not invariant under the Lorentz group of special relativity (and electromagnetic theory in empty space), it is generally believed that they imply all the laws of chemical reaction.

As a result of these and other discoveries, one can claim that all of physics has been reduced to a branch of applied mathematics. To paraphrase Lagrange, could we but integrate these partial differential equations, we should be able to predict all physical phenomena with as much precision as Newton's Laws enable us to predict the phenomena of celestial mechanics. The main mathematical difference is that, whereas Newton was dealing with ordinary differential equations, continuum physics in general involves partial differential equations.

To perform this integration is already routine for heat conduction in slabs and shells. Moreover variations in physical properties (e.g., conductivity and specific heat) with position and temperature pose no great problem. Whereas classical analysis relied strongly on linearity and homogeneity, mild nonlinearities and inhomogeneities are automatically taken care of by modern numerical methods.

For the applied mathematician of tomorrow, one of the greatest challenges is to fulfill this implicit promise, for example in aerodynamics and chemistry. Using mathematical analysis and the computer, the ultimate objective would be to replace wind tunnels and chemical test tubes by skillfully organized mathematical equations, capable of being solved economically to the needed accuracy on a computer. I shall discuss next the prospects for progress towards this objective during the next few decades.

\section{Scientific Computing}

The methods of classical mathematical analysis achieved near miracles in solving problems involving special geometries and linear partial differential equations. This progress depended on techniques such as the "separation of variables" and expansions in series of products of functions of one variable which we know cannot be applied effectively to general geometries or to nonlinear partial differential equations. 
Numerical mathematics has no such limitations in principle. Indeed, already before World War I, Adams, Runge, Størmer and others had developed simple and practical numerical methods for solving general systems of ordinary differential equations. A century earlier, Gauss, having in mind applications to geodesy as well as astronomy, had invented Gaussian elinination, Gaussian quadrature, and the method of least squares.

In the past 30 years, the development of high-speed computers with large "memories" has enormously increased the range of economical application of known computing methods, and stimulated the development of new ones. The resulting revolution in the art of scientific computing has many basic implications for the future of applied mathematics, and these will be central to the rest of my talk.

Linear Systems. A good example of the kind of improvement that has occurred in the past 30 years is provided by the problem of solving large linear systems, of the form

$$
\begin{aligned}
& a_{11} x_{1}+a_{12} x_{2}+\ldots+a_{1 n} x_{n}=b_{1} \\
& a_{21} x_{1}+a_{22} x_{2}+\ldots+a_{2 n} x_{n}=b_{2} \\
& a_{n 1} x_{1}+a_{n 2} x_{2}+\ldots+a_{n n} x_{n}=b_{n}
\end{aligned}
$$

We can rewrite (3) in matrix notation as $A x=b$. Gauss already solved many such systems for $n$ in the range 5-10. However, such computations were slow and had to be done with great care. To solve a single such system in 10 unknowns would have taken hours and cost around $\$ 10$ even in 1945. Whereas on a large modern computer, it takes a fraction of a second and costs around $0.03 \phi$ or so.

Boundary Value Problems. Likewise, although good difference approximations to wide classes of partial differential equations were known before $1940,{ }^{14}$ to achieve adequate accuracy even for Dirichle problems required solving linear systems (3) having several hundred unknowns. To solve one such system took months of work by a capable graduate student; hence the cost was of the order of $\$ 1000$ per case.

In 1950, David Young showed how to automate some of these procedures by a "successive overrelaxation" (SOR) method. Using SOR on today's computers, one can solve in seconds a wide variety of linear boundary value problems involving functions of two independent variables. On a $30 \times 50$ mesh, the cost might be around $\$ 5$. The seepage of petroleum in reservoirs and the diffusion of neutrons in nuclear reactors are two industrially important processes treated in this way. I shall discuss the applica-

${ }^{14}$ See L. V. Kantorovich and V. I. Krylov, Approximate Methods of Higher Analysis (Interscience 1958; first Russian edition, 1936). 
tions of mathematics to these processes further in the section on Engineering Computing.

Since 1950, many other ingenious methods have been invented for solving such problems: conjugate gradient, alternating direction implicit (ADI), strongly implicit, fast Poisson, and symmetric SOR (SSOR) to mention only a few. ${ }^{15}$ During the next 5 years or so, these should become generally available in the form of a LINPACK (linear systems solving package) currently being developed by the Argonne National Laboratory in cooperation with several universities. A similar EISPACK for solving eigensystems is already available; it will find all the eigenvalues and eigenvectors of a $100 \times 100$ matrix at a cost of around $\$ 1$.

Spectacular and important though these advances are, it must be remembered that most scientific problems involve at least three independent space variables, and often time as well. Computing costs must be reduced by another factor of $10^{6}$ before their solution can be achieved systematically by standard methods. Although "parallel processors" with many arithmetic units may contribute substantially to achieving this goal, we should not count our chickens before they are hatched!

To be more specific, Fourier's equations only describe heat conduction approximately (neglecting variations in specific heat with temperature); convection is much more important in fluids, and its scientific description involves all the complications of turbulent flow, whose detailed description would require using billions of mesh-points.

Similarly, one is typically interested in the propagation of electromagnetic waves through regions, often inhomogeneous, whose diameter is millions or billions of wavelengths. Even using geometrical optics as a first approximation, it will be very difficult to model such situations in any detail on a computer.

Likewise, the two-body problem for Schrödinger's equation involves an unknown function of six, and the three-body problem one of nine independent variables. To treat them with satisfactory accuracy will require extraordinary ingenuity.

In short, it can easily become prohibitively expensive to gratify our scientific curiosity. Although dramatic advances have been and will continue to be made in scientific computing, for every question that can be answered within a given computing budget, there will always be 10 that cannot - even if the underlying physics is well understood in principle.

\footnotetext{
${ }^{15}$ For SOR and ADI, a good reference is Varga [37]: for Algol versions of the EISPACK programs, see [35].
} 
I think the greatest use of applied mathematics during the next 50 years will be for engineering computing: the development of computer models of reality which are adequate for design purposes. This is a fine art, and by no means a science.

To illustrate my meaning, consider the use of SOR in nuclear reactor design. Here a continuous range of neutron energies extending from 0.03 to $5 \times 10^{6} \mathrm{ev}$ is typically simulated by four neutron "energy groups"; the finite mean free path, coherent scattering, and many molecular (chemical) influences are neglected or very crudely approximated; and two-dimensional horizontal slices are spliced together by intuition and experience to "predict" three-dimensional flux distributions in a most ad hoc way.

Or again, consider the unknown macroscopic variations in permeability that must occur in oil-bearing sands, not to mention unknown details of boundary and interfacial configurations. These must be conjectured in practice from a subtle and complex analysis of the available computational and empirical evidence.

Even in the much simpler geometrical problem of representing and designing automobile body surfaces, the smoothing, "sweetening," and piecing together of various design elements involves a complex interplay of digital, continuous, probabilistic and aesthetic considerations that can only be coordinated by a skilled and perceptive human being!

In trying to understand why such drastically oversimplified models can be reliable in practice, I have come to the conclusion that conservation laws play a fundamental role. Thus the total number of neutrons change only through fission or absorption; the total volume and density of oil change only when the oil is forced into an oil well by pressure variations; and so on. A much more fundamental example concerns the mechanical equivalent of heat: the principle of conservation of energy (chemical, mechanical, thermal, and electrical) in its wider form The "irreversible" tendency of dissipative effects to convert mechanical and electrical energy into heat (the "second law" of thermodynamics) is another basic example.

Another potent tool is provided by empirical correlations, typically representing an average of many statistically varying quantities. The pressure exerted by a gas is a classic example; much less reliable, but often good to within 5 or 10 percent, are the empirical friction "laws" for fluid flow. Empirical laws of heat transfer for turbulent one- and two-phase coolant flow in pipes provide a still more complicated example.

To apply such "laws" successfully, it goes without saying that an engineer must have a wide experience not only concerning their statistical reliability, but even more importantly concerning the qualitative phenomena (e.g., "film" and "subcooled" boiling) responsible for the changes of state etc. being considered. In short (see the end of sec. on Hydraulics and 
Hydrodynamics), he must exercise engineering judgment in interpreting his computer output.

Whether or not he calls himself an applied mathematician, therefore, a person developing useful mathematical models to help in solving engineering design models should be familiar with a wide range of physical and empirical laws, as well as with the methods of mathematical analysis, discrete approximation, and computer science. Conversely, I think there will be a great need for such persons in the decades ahead.

\section{Mathematical Economics}

Quantitative (i.e., mathematical) methods are playing an ever-increasing role in theoretical economics. ${ }^{16}$ Today, every educated person has heard about "game theory," "linear programming," and "optimization," and has a dim perception that such mathematical concepts play a central role in the analysis of widely accepted economic models.

He usually also realizes that the mathematical techniques used to "maximize the payoff" (at least in the model!) can be very ingenious and sophisticated, and may require a large computer for their effective implementation. Finally, he is aware that they were invented relatively recently, and is (rightly) suspicious of the validity of the output.

$\mathrm{He}$ is perhaps less conscious of the extent to which mathematics itself has already been enriched by the introduction of such new concepts, and will probably continue to be in the future. This is partly because, although mostly invented by mathematicians, they do not fit well into the now conventional framework of pure mathematics constructed by the famous M. Bourbaki, and are therefore excluded from the normal graduate program.

This exclusion seems to me most regrettable: the theories involved are mathematicaliy unimpeachable; current and future thinking by mathematical economists can only enrich mathematics.

The extent to which it will enrich our economy is, of course, another matter. As always, arithmetic and realistic logic are the most important branches of applied mathematics, and I find it astonishing that so many academic economists have climbed aboard the "growth rate" bandwagon, when it is so obvious that a 7 percent economic growth rate cannot be maintained for even 2 centuries. Currency inflation is the only area where this growth rate can be maintained indefinitely (by redefining units), and a projected 50 billion dollar deficit for the third consecutive year suggests a firm desire to prove it!

${ }^{16}$ For some pioneer views, see [5] and [13]. 
Surprisingly also, both economists and accountants seem to have largely ignored the simple mathematics of inflation (except perhaps from a speculative st andpoint; see [23]).

Consider the following, not atypical example. A corporation amortizes $\$ 100$ million of new capital investment over 5 years, realizing a before tax paper "profit" of $\$ 50$ million. Of this, it pays out $\$ 25$ million in taxes and $\$ 20$ million in dividends, retaining $\$ 105$ million for capital replacement. Due to 9 percent inflation, this now costs $\$ 150$ million. The investing public must therefore subscribe $\$ 25$ million besides reinvesting all their dividends (of which $\$ 8$ million has incidentally been taken in taxes) to keep the company in business. How much profit has the company really made, and how long will a "capitalistic" system using this kind of accounting survive?

Related Fields. Although mathematical economics is more glamorous, and some of its most brilliant theorists have been rewarded by the new Nobel prizes financed by Swedish banks, the related fields of operations research [20], management science, and actuarial science [9] should be mentioned, as using similar mathematical techniques and offering many good career opport unities. ${ }^{17}$

Another fascinating related field is that of "social forecasting" and global resource allocation [19]. ${ }^{18}$ Here we are confronted with an extreme example of the main objection to applying mathematics to the social sciences: one simply does not have enough data to make mathematical models reliable.

\section{Mathematical Biology}

Mathematical biology is another difficult area offering infinite challenge to ambitious applied mathematicians. Here tribute should be paid to d'Arcy Thompson's pioneer classic [25], in which geometry and dimensional analysis were suggestively applied to correlate and explain facts about the possible sizes and speeds of locomotion of animals, and the symmetries of patterns occurring in Nature. A valuable sequel to this is $N$. Rashevsky's Mathematical Biophysics (Univ. of Chicago Press, 1938), which emphasizes physical (and not just geometrical) interpretations.

An imaginative recent contribution in the spirit of d'Arcy Thompson is Réné Thom's well-publicized (by C. Zeeman) theory of "morphogenesis." 19 However, I think that this is a fundamental contribution to pure

\footnotetext{
${ }^{17}$ The combined membership of TIMS and ORSA alone is about that of the American Mathematical Society.

${ }^{18}$ See Proc. Nat. Acad Sci. 69 (1972), 3828-3831.

${ }^{19}$ Réné Thom, Stabilité Structurelle et Morphénèse (Benjamin, 1972). Questions of embryonic development are treated from a qualitative standpoint.
} 
combinatorial topology. From an applied standpoint, it greatly oversimplifies biological reality. Thom refers to morphological transformation as if they were purely geometrical deformations of homogeneous media, whereas real biological cells have a very complicated internal structure and a skin (membrane).

A very different combinatorial approach to the related problem of DNA coding has been proposed by Crick; a review of work based on this approach is given by Golomb in [30, vol. XIV, pp. 87-100], where the relevance of Paley's theory of Hadamard matrices ${ }^{20}$ is explained.

Deeper technically than any of the above, because it builds on a solid foundation of theoretical fluid mechanics (see sec. on Continuum Physics), is Lighthill's brilliant and versatile mathematical analysis in [14, Part I] of the known modes of animal propulsion and locomotion. In [14, Part II], he makes a similar study of human respiration and blood circulation. ${ }^{21}$

Penetrating as these studies are, they do not reduce the aspects of biology treated to purely mathematical questions. Indeed, they cannot until the condition for flow separation (which determines drag and influences lift) in pure fluid dynamics has been predicted from first principles (i.e., from the Navier-Stokes equations). Moreover [14] ignores the rich biochemical phenomena that control muscle contractions and muscle tone, and the psychological problems of coordination and control (e.g., in flying, swimming, and breathing).

Another beautiful application of mathematics to biology concerns the Hodgkin-Huxley theory of nerve impulses in squid axons. This is reviewed in [32, chap. 6]. By including electrochemical factors in their model, Hodgkin and Huxley have transcended the limitation to mechanical models of most workers in the field of mathematical biology. I suspect that this will set a new trend, and that the past emphasis on mechanical models stems from their greater familiarity (see sec. on Continuum Physics, beginning). To understand the liver, kidney, and other bodily organs, chemistry must surely be invoked!

\section{Mathematics and Psychology}

Among biological attributes, intelligence is surely the highest, and I shall conclude with some observations about mathematical models of the mind or "psyche"-i.e., about mathematics and psychology.

The great pioneer in this field was Helmholtz, who analyzed with great care our senses of hearing [8] and vision-i.e., how we perceive sound and

\footnotetext{
${ }^{20}$ R. E. A. C. Paley, J. Math. Phys. 12 (1933), 311-320.

${ }^{21}$ The botanical analogue of blood in sap, which rises under capillary and osmotic pressures (and tensions) up to 40 atmospheres. See PLANTS and PLANT SCIENCE, Enc. Britt., 1971 edition.
} 
light. Time has shown many of his ideas, immensely suggestive as they were, to be greatly oversimplified.

Thus in the process of hearing, sound waves are transmitted by an extremely complex process from the eardrum to the cochlea, basilar membrane, and associated hair cells. The physiology of all these is very complicated, and nobody really understands how they (and the connecting nerves) can sort out musical notes, and combine the binaural pressures $p_{1}(t)$ and $p_{2}(t)$ to produce stereophonic sensations. Von Békésy gives a critical, not very mathematical review in [32, chap. 7$]$; he does not try to explain how musical training heightens perception.

As regards vision, Rashevsky's student Landahl has produced a new theory of color vision, while Minsky and Papert ${ }^{22}$ have made a very clever analysis of "perceptrons," a class of artifacts designed to recognize patterns, an essential capability of human intelligence. The ultimate goal of Minsky and Papert is the construction of a robot, capable of such human functions as building a brick wall, assembling a radio, or even proving mathematical theorems.

Actually, in his famous 1937 paper, Alan Turing already designed an idealized computer (a so-called "Turing machine"), capable in principle of proving any theorem that could be established by the usual assumptions of mathematical logic. Moreover robots are gradually being introduced into automated production processes.

Intrigued by the idea that computers might replace mathematicians as theorem provers, I made a careful analysis 7 years ago of the practical problem of proving mathematical theorems by computer. My conclusion $[1,16]$ was that man-computer symbiosis was the key to progress, and that:

...To make this symbiosis truly effective, our society will need also applied mathematicians, both as numerical analysts to keep track of the approximations which are made in modeling continua and, even more important, to relate the output of computers to the scientifie and engineering problems which they are intended to solve.

I am still of this opinion, ${ }^{23}$ and I hope that Alan Perlis will present further evidence shedding light on this and related questions in his talk.

${ }^{22}$ M. Minsky and S. Papert, Perceptrons (MIT Press, 1969); see also F. Rosenblatt, pp. 63.96 in Self Organizing Systems (Pergamon).

${ }^{23}$ Since this talk was given, the recent apparent computer-aided solution of the four-color problem bv Haken and Appel would seem to support my conclusion. 


\section{References}

A large number of older references have been included, to set the field of Applied Mathematics in a larger historical perspective.

[1] Birkhoff, G., "Mathematics and Psychology," SIAM Reviews 11 (1969), 429-469.

[2] Birkhoff, G., "Mathematics and Computer Science," American Scientist 63 (1975), 83-91.

[3] Birkhoff, G. and Bartee, T. C., Modern Applied Algebra (McGraw-Hill, 1970).

[4] Chandrasekhar, S., "Transfer at Radiation in Stellar Atomspheres," Bull. Am. Math. Soc. 53 (1947), 641-711.

[5] Fisher, Irving, "The Application of Mathematics to the Social Sciences," Bull. Am. Math. Soc. 36 (1930), 225-243.

[6] Fry, Thornton C., "Industrial Mathematics," Am. Math. Monthly 48 (1941), Special Supplement (38 pp.).

[7] Gibbs, J. Willard, Scientific Papers, 2 vols. (Yale Univ. Press, 1906; Dover, 1961).

[8] Helmholtz, H. L. F., On the Sensation of Tone (Dover, 1954; original German ed., Vieweg, 1863).

[9] Henderson, Archibald, "Life Insurance as a Social Science and as a Mathematical Problem," Bull. Am. Math. Soc. 31 (1925), 227-252.

[10] von Karman, Th., "The Engineer Grapples with Nonlinear Problems," Bull. Am. Math. Soc. 46 (1950), 613-683.

[11] Kraus, C. A., "Present Status of the Theory of Electrolytes," Bull. Am. Math. Soc. 44. (1938), 361-383.

[12] Klein, Felix, Entwicklung der Mathematik im 19ten Jahrhundert, 2 vols. (Springer, 1926; Chelsea, 1960).

[13] Leontief, Wassily, "Mathematics in Economics," Bull. Am. Math. Soc. 60 (1954), 215-233.

[14] Lighthill, Sir James, Mathematical Biofluiddynamics (Soc. Ind. Appl. Math., 1975).

[15] Luce, R. D., Bush, R. R., and Galanter, E., eds., Handbook of Mathematical Psychology (Wiley, 1963).

[16] Luce, R. D., Bush, R. R., and Galanter, E., eds., Readings in Experimental Psychology (Wiley, 1965).

[17] Mayer, J. E., "Structure of Simple Fluids," Bull. Am. Math. Soc. 62 (1956), 332-346.

[18] Maxwell, J. C., Electricity and Magnetism, 2 vols., 2d ed. (Clarendon Press, 1881).

[19] Meadows, D. H., Meadows, D. L., Randers, J., and Behrens, W. W. III, The Limits to Growth (Universe Books, 1972).

[20] Morse, P. M., “Mathematical Problems in Operations Research," Bull. Am. Math. Soc. 54 (1948), 602-621.

[21] von Neumann, J., Collected Works, 6 vols. (Pergamon Press, 1961-1963).

[22] Poincaré, Henri, Foundations of Science.

[23] Samuelson, Paul H., "Mathematics of Speculative Price," SIAM Reviews 15 (1973), $1-42$.

[24] Stone, M. H., "Mathematics and the Future of Science," Bull. Am. Math. Soc. 63 (1957), 61-67.

[25] Thompson, D'Arcy W., On Growth and Form, 2d ed. (Cambridge Univ. Press, 1952).

[26] Timoshenko, S., History of Strength of Materials (McGraw-Hill, 1953).

[27] Weyl, H., "Ramifications of the Eigenvalue Problem," Bull. Am. Math. Soc. 56(1950), 115-139.

[28] Wigner, E. P., "Symmetry Principles in Old and New Physics," Bull. Am. Math. Soc. 74. (1968), 793-881.

[29] Woodward, R. S., "The Century's Progress in Applied Mathematics," Bull. Am. Math. Soc. 6 (1900), 133-163. 
[30] Proc. Symposia in Applied Mathematics, vols. I-XX (Amer. Math. Soc., 1948-1966 and 1974).

[31] SIAM-AMS Symposia in Applied Mathematics, vols. I -VI (Amer. Math. Soc., 19671973).

[32] Waterman, T. H. and Morowitz, H. J., Theoretical and Mathematical Biology (Blaisdell, 1965).

[33] Encyklopadie der Mathematischen Wissenschaften (Teubner, 1904).

[34] Whitaker, E. T., History of the Theories of the Aether and Electricity (Longmans, Green \& Co., 1910).

[35] Varga, R. S., Matrix Iterative Analysis(Prentice-Hall, 1962). 
Professor Alan J. Perlis is Higgins Professor of Computer Science at Yale University. He was a pioneer in establishing the field of computer science as an academic discipline. He has contributed in a major way to the theory of programming and was one of the first to develop automatic programming procedures. He has been president of the Association for Computing Machinery and head of the Computer Science Department of Carnegie-Mellon University. He is active on various computer-related Government committees, serving on the NSF computer activities committee, the NAS Computer Science and Engineering Board, and others. As the first Turing Prize Lecturer, Professor Perlis spoke on the subject of the "Synthesis of Algorithmic Systems." 


\section{COMPUTERS IN SCIENCE AND TECHNOLOGY}

\section{Introduction}

It is fashionable to use the computer as a pivot on which to treat movements in our physical and economic world, even in our social world. The computer is used as model and as metaphor. Every part of science and technology has been infected and altered by it. The computer is the atom smasher, microscope and telescope of the economic and social sciences. A more adequate historian of science than I will be required to measure properly the integrated impact of this device on the progress and plans of those areas where it has become widely used.

The domestication of the computer has been so rapid that one despairs of adequately characterizing its role or predicting its future. No other inanimate device yct created by man shares this chameleon property of both blending into the background and usurping the foreground of our diverse physical and intellectual interests.

A misconception still shared by many is that the compter is merely another multipurpose tool, a boy scout knife of our modern symbol world. A tool it certainly is, but it is much more. A more proper view of this mixture of metals and semiconductors is as a (static and dynamic) projection of the mind, albeit a currently quite primitive projection. Many computer scientists have chosen to characterize its role in that way.

The literature is replete with descriptions of applications of computers in the physical and social sciences and technology. Little point would be served by merely describing or listing some of these applications in this paper. Instead I shall provide a series of mini-essays on computers that may prove enlightening.

\section{How the Scientifically Trained Public Views the Computer}

The computer affects scientists and science. The following points reflect the prevalent views held at the present time by scientists. Most scientists and technologists are aware that they must be able to adapt the computer to their activities. Regardless of the degree of computer involvement in their affairs, the computer is seen as only a tool, though admittedly a very flexible one. This tool is to be bent to the purposes at hand much as any other technological stimulant. However, the notion is resisted that the computer has any deeper effect on the work scientists do than to improve 
efficiency and to increase rates of production and change. It is recognized that the device is evolving. Both its internal functions and resources are being augmented rapidly, while its size and per operation cost are decreasing rapidly. Unlike the other high technology tools used in science and technology, the computer is the main product of a big business, and it is penetrating commercial activities in ways identical to those useful to science and technology. Furthermore, the computer business is an employer of large numbers of scientists and engineers who work at the research boundaries of their respective fields.

It is known that an area of intellectual interest called computer science has sprung up. Scientists are both chauvinistic and tolerant when comparing their own science to others. Computer science, it is conceded, has a right to exist even though there is, as yet, precious little science in it. Things would be so much better if computer scientists would concentrate on making it easier for everyone to get useful work from a computer instead of studying or attempting to define the scope of the field. In any event the core content of computer science is adequately covered by the classical sciences: physics, mathematics and economics.

Our society probably could not function at the accustomed levels of efficiency and choice without the computer. We are not sure whether this is good or bad, or if it should be considered in ethical terms at all.

We do take pride in the fact that the computer is primarily an American invention, and its widespread exploitation has been pioneered in this country. A vital technology, running the full gamut of managerial and entrepreneurial concerns, has been created.

Computers and their programs often refuse to run. The consequences may be enormous in loss of lives, time, and dollars. Our dependency on the machine does not necessarily improve our control over its operation. Frustration is a word that one associates quite naturally with the computer: everything is possible, but nothing of interest is easy. It is difficult to curb one's appetite for computation, it being easier to know what can be done than to understand what should be done. Even when we know precisely how to organize a task for the computer, we may not be able to program it successfully. Logistics, unforeseen second-order effects, and changes in designs intrude. Any attempt to perform or manage a reasonably large programming task makes us painfully aware of how necessary it is to acquire and master a disciplined methodology of programming.

Above all we do wish that the computer field would slow its frantic pace of change. We, who have not exhausted the usefulness of our present computers, are understandably upset by order-of-magnitude improvements whose exploitation may require extensive rearrangements in our problemsolving techniques. 


\section{How Much Have Computers Changed in 30 Years?}

The attached figures, due to E. David [1], give some indication of size and cost changes. The number of (stored program, electronic) computers has risen dramatically - 250,000 would not be a bad estimate for their current population. There has been a slow growth in augmented functions-addressing flexibility, index registers and floating point, a rapid growth in primary and secondary storage capacities and brute speed; an enormous increase in choice of input/output $(I / O)$ modes. There is in the computer industry a ruthless sustained drive to eliminate any and all bottlenecks that impede the most efficient use of components. Fortunately there is an almost inexhaustible supply of physical phenomena and materials from which to fashion componentry. Production devices quickly follow prototypes; a 2- to 5-year gestation period is normal. The computer is an instrument whose destiny is unfulfilled. User wishes, entrepreneurial possibilities, and component feasibility mutually reinforce a rapid evolution. We know the growth curves will flatten out, but at what levels of capability will this occur?
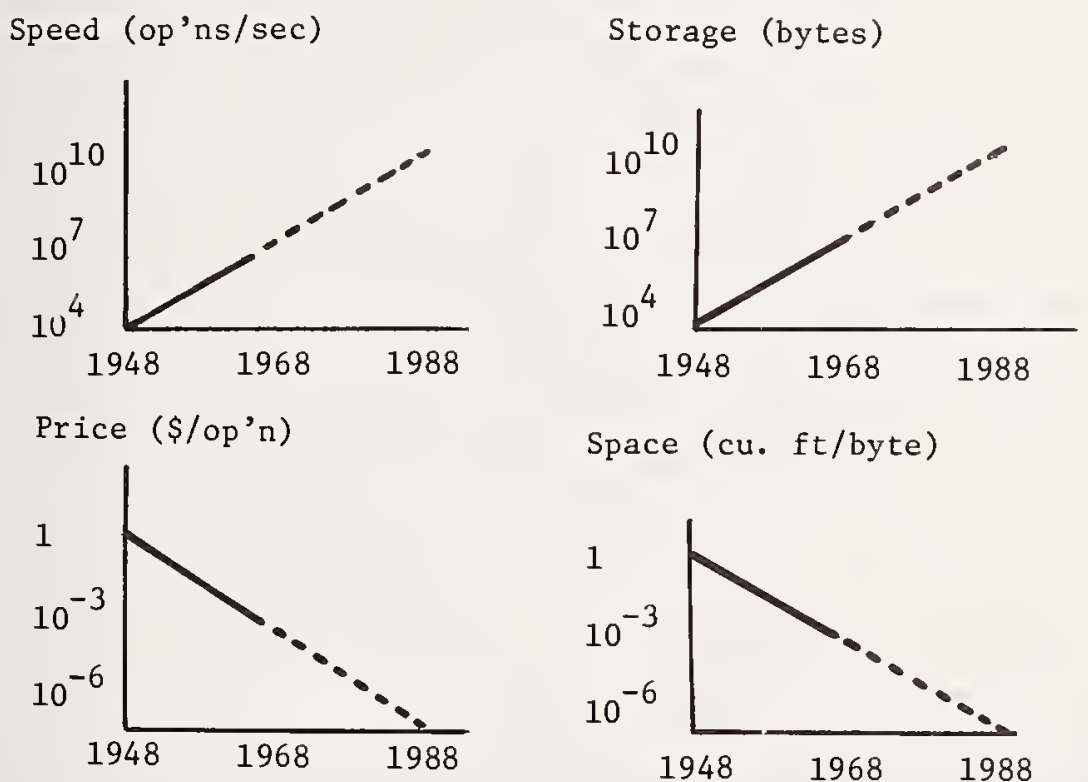

Space (cu. ft/byte)

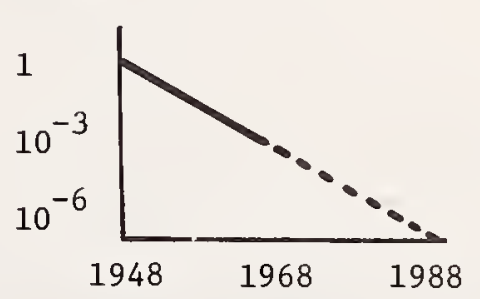

Figure 1. Some historical and projected trends associated with computer development.

Nevertheless, the computer has not really changed its fundamental nature and organization since the EDSAC went on line in Cambridge, England in 1949. The nature of the machine is still dominated by the fetch-execute control cycle and a program, more or less fixed in structure and content, repetitiously analyzing batches of data. The design, sometimes called 
the Von Neumann model, has proved durable and enormously useful. We have provided pre-processors and post-processors to cushion our contact with the machine. We have interleaved and synchronized parts, networked devices and defined any number of virtual machines resident on our hardware. The basic model endures, and alternatives have become difficult even to imagine. The computer is a stable species, though its physical dimensions seem to be of unlimited variability.

The existence of a simple model, subject to enormous numbers of useful variations, suggests some important pedagogic approaches to education, both formal and informal, about the computer. Where the audience is heterogeneous in background and interest, one must commence the understanding of the computer with its commonly perceived epidermis, the high level language and the virtual machine. Familiarity and pleasure with performance at this level by the student must be followed by a detailed sequence of simplifications of both language and machine. Each step introduces a new level of coding rigidity with a consequent increase in conceptual machine simplicity. Ultimately one reaches the level of microcode and micromachine. At this point logical design can be appreciated and sim. ple circuits studied.

Such microscopic explorations are necessary to provide an Olympian overview so necessary for taking advantage of the flexibility laid before us by computers. One grows to appreciate many of the factors that determine the trajectory of the boundary between hardware and software. Nowhere is this appreciation of the computer more valuable to us than when we move to use the computer in "frontier" problems. I refer to problems where time, storage capacity and machine organization are critical, shaping our problem-solving methods and even determining whether computer solution is feasible at all. Every user of the computer, certainly every programmer, should have the grand design of decomposition and synthesis etched into his skull, even if he is a tyro in computer technology or only dimly aware of the laws that govern choice of problem-solving method and machine.

\section{Appreciation of the Computer}

While the computer remains a stable species, our appreciation of what can be done on it has grown enormously in the past two decades. The first appearance of the computer in many important scientific, technical and social applications is still taking place. The device's universality is gaining more and more support, and society is increasingly opting for those developments where the computer can be most gainfully employed.

We note that the introduction of the computer rarely leaves it as a weakly connected node in a problem chain. Actual use of the machine in. 
duces changes in its embedding environment separate and different from those predicted a priori. The act of computer programming induces a level of formalization that suggests, often requires, avenues of change. A system of communication is defined that must be managed (and exploited), for example, patterns of language and documentation. Using a computer is as stepping through Alice's looking glass. In this new world syntax dominates semantics, means dominate ends, and everything is a special case of something more general. The power to act through the computer seems limitless since the energy required is roughly at the verbal level. However, it is only the power to act randomly (hence not at all) that is so cheap. Optimal extension of a computer's role requires personal and managerial discipline and organized thought that can only be obtained by the expenditure of time engaged in arduous creative thought. Only the early steps of computer application, paid for by our previously acquired scientific and technical expertise, come cheaply.

There are ways by which we can prepare ourselves for the increased involvement of the computer in our activities. We can become adept at thinking in terms of creating and managing computer applications. We think in terms of process and represent our subjects of discourse as "symbol systems" [2]. The computer is no longer seen as an obedient and passive tool: it is the host to, and manipulator of, our symbol systems. Our processing is then almost totally within the system, and topical reference to the real world occurs only at the onset and conclusion of our enterprise.

Mathematics is probably the most widely used and venerable symbol system. Applied mathematics is the collection of symbol systems customarily used to model continuous processes, for example, by differential equations. These systems existed and flourished for at least two centuries before the computer. This device has not fundamentally altered applied mathematics, though it has fostered reorientation in many of its aspects. Thus we have now come to accept a wider range of meaning for the term "solution of an applied mathematical problem."

The similarity of all symbol systems in the computer, regardless of their source, permits us to conceive of, and manipulate, systems that have no significant component within classical applied mathematics. A most important class of such systems is that loosely labeled "artificial intelligence" systems. Their programs do not guarantee solutions to problems posed. Their iterative components do not necessarily converge nor can we isolate general conditions of either convergence or divergence. These processes employ heuristic search and behave primitively much as we do when we wander through strategies to solve huge combinatorial problems.

Let us consider a specific example, a symbol system to perform the synthesis of organic compounds [3]. No one has been able to isolate an algorithm that will either specify the synthesis of an organic compound $\mathrm{X}$ or show that none such exists. We do not know if such an algorithm is even 
possible. However, we do know that symbolic systems for defining synthesis exist in the minds of organic chemists.

I selectively quote from Gelernter et al.: "...the techniques and methodology of artificial intelligence in general, and heuristic programming in particular, had matured to the point where problems of substantial content and of interest in themselves ought to be selected by those engaged in that activity, e.g., organic synthesis discovery."

It is believed by Gelernter and his associates that the difference between a feasibility demonstration and a system of general utility to chemists is one of degree rather than kind.

But of greatest importance to the future development of such and other symbol systems is the observation that the mind adept at, and experienced in, creating AI symbol systems is necessary to provide the skeletal organization and control in order that a threshold of competence be reached by such a programmed system. Knowledge of the subject field alone (here organic synthesis) is not enough. Put another way, the threshold can be reached by computer scientists (my first use of that term) consulting with chemists, but is not likely to be reached by chemists employing computer programmers. The organizational, control, and coding is sues are too fundamental and pervasive to be treated as appendages to the organic synthesis paradigms. Instead it is the other way around! Gelernter found that the procedures for the generation of synthesis solutions do not differ vastly from the procedures he used a decade earlier in a computer program for theorem proving in Euclidean geometry. Thus it was his experience with the geometry problem that emphasized the need to have linear symbolic formulae for representing compounds so that their machine processing could be adequately and efficiently performed. That which is a notational convenience to the chemist is a piece of essential structure to a program. Recognition of this necessity and search for its solution is a dividend arising from concern with symbol systems, per se. The invention of linguistic structures relevant to a problem domain, that are machine processible, is a standard approach of the computer scientist, and he invents in similar ways whether he is processing theorems, organic compounds, music, natural language, or what have you.

I certainly do not wish to imply that matters dependent upon the subject being studied are unimportant. Far from it. Knowledge of chemistry is critical to the establishment of chains of useful goals and pruning of search trees, for these are not syntactic issues, but semantic ones. However, until such time as experience and insight into construction of growing symbol systems become working tools of the scientist and technologist, the com puter scientist must dominate at least the initial development of these very important symbol systems.

Of course one may ask, why build such systems at all? Why lay bare our intuitive problem-solving genius? One answer might be that depen- 
dence on the work of problem-solving geniuses is getting to be too great for the population of geniuses we have on hand. I doubt that reason. A more compelling reason for spawning such systems is that each such exercise adds to our own stock of knowledge about the problem-solving abilities of the mind. The only bound on that stock of knowledge that we may tolerate is our ignorance, and hence the bound is reducible. Any other proscription is childish.

\section{Computer Science}

Computer science is the study of the phenomena arising around computers [4], and in particular, the study of symbol systems that spring into existence on computers in response to phenomena. While many classical disciplines deal with symbol systems, only computer science is concerned with the dynamics of all such systems, from the phenomena that conceive them, the programs that represent them, the applications and theories that use them, and the machines that we create and stuff to hold and manipulate them.

There have always been algorithms, abstract programs, but only with the advent of the computer has their number and diversity been sufficiently rich to suggest a need for studies to classify and approximate algorithms by other algorithms. Since Turing's epic work in 1937 we have known of algorithms we cannot describe, that is, that do not exist. However, of even greater importance is our growing recognition that certain problems have algorithms, but cannot have any that are useful. All these algorithms are exponential in the use of some key resource. There are other algorithms that approximate exponential algorithms, i.e., give results close to optimal, but are only polynomial in their use of a resource. We are witness to the birth of a new numerical analysis, called concrete complexity theory, spawned not from classical applied mathematics but from combinatorics. Complexity theory is a fundamental topic in computer science.

The creation of symbol systems, themselves a fundamental topic in computer systems, leads to linguistic inventions that form the basis of software, materialized on hardware. The definition of the software-hardware interface is a fundamental topic of computer science.

Among the symbol systems we create are those whose function is the generation of other symbol systems. We call this area of synthesis automatic programming. We must be careful to understand the range of meanings this evocative term is applied to. At one end is the historic compiler that translates programs written in a user-preferred language such as FORTRAN into machine code programs. FORTRAN is a user-preferred language because the user may omit irrelevant details and specify in a convenient format those details that must be included to fix the intent of his 
program. At the other end of the spectrum is the ideal user language, statements of wishes that are transformed into a computer program. Needless to say a symbol system capable of such transformations is well beyond our present capabilities. It is part of the lusty optimism that flowers around computers that pushes us to search for programs with properties that, in more ancient times, were exclusively held by "djinns" in lamps. Some progress can be reported. A program has been written [5] that produces a symbolic machine assembly language and its assembler for a computer given its formal description in a language called ISP. Work is underway on the production of programs that create the servicing programs for the peripherals (tapes, disks, terminal, printers, etc.) of any computer for which a formal description of such peripherals exists. A natural mediumrange goal of this automatic programming effort is the automatic production of compilers for arbitrary (new) machines in ways that take advantage of machine idiosyncracies. The study of, and creation of, programs that do what programmers do is a fundamental part of computer science.

\section{A Chronology}

Stretched behind us is a history of computers that is barely 30 years old. While some of us have witnessed this entire passage as participating adults, most people now engaged in computing have not. In table 1 , I have listed a brief chronology of events that, in my opinion, have enormously influenced the developments we are witnessing. It goes without saying that there are events, omitted from this list, that have contributed as much (or more) as the inclusions. The dates are chosen to identify an interval of time rather than to pinpoint a year. No entry more recent than 1971 is included, not because nothing of import ance has occurred since then, but because so much of equal importance is taking place.

It is natural that the ENIAC should head the list.

ACM from its inception has been the technical society for computer people, and its meetings and journals have provided the necessary forums for displaying the technical growth of the subject and as an essential avenue of communication between computer scientists and technologists.

The ARPA (Advanced Research Projects Agency of the Defense Department) research program stimulated the development of computer science as an independent subject setting its own research and development frontiers. The funding levels provided by ARPA were sufficient to stimulate artificial intelligence, graphics, speech and time sharing to levels deserved by the areas but not hitherto possible.

Sketchpad, a graphics program on TX-2 at Lincoln Laboratories, opened up the possibility of computer-produced drawings. Since that first program there has been a steady development of programs and equipment 
(circa)

1947 The first electronic divital computer (ENIAC)

1948 The founding of the Association for Computing Machinery (AC.M)

1948 The start of modern numerical analysis

1950 The first commercial computer

1952 The first programming aids

1956 FORTRAN

1957 The beginning of artificial intelligence

1959 The first international computer conference (IFIP)

1960 ALGOL60

1962 ARPA research program in computer science (\$16 million annually and rising)

1963 Sketchpad

1965 Time sharing

1965 The first failure: Natural language translation by computer

1967 Artificial intelligence approaches practical utilization-Dendral, Robotics

1969 The second failure: IBM: TSS for the $360 / 67$

1970 The ARPA network

1971 The handheld solid state calculator

capable of producing pictures in three-dimensional projected form, in color, exhibiting simulated texture and shading, and animation, all of the highest quality.

A scientific area matures as much through recognition of failure as through pride in success. Performing natural language translation on computers was an early goal of linguists and computer specialists. Adequate federal funding and computer access were provided. Within a few years good progress in syntax analysis was reported. Several excellent parsers, both of Russian and English, were reported. Machine-readable dictionaries were prepared. However, programs could not be created to cope with meaning. Translation depended upon context, the semantic environment in which text was embedded, and programs did not "know enough" to recognize environments so that sense could be preserved under translation. This was true even for technical material largely divorced from literary allusions. Some have interpreted the failure as evidence of an inherent 
limitation of computer programs, the definition of a boundary beyond which it is futile to expect programs to go. I suspect that a willingness to accept this view as the ultimate state of affairs is as prone to error as was the intemperate optimism of computer people when they began their work on language translation. Work on language understanding programs, now underway, gives some evidence that programs can be written that, in a limited sense, understand a context and can extract meaning from textual exchanges about the context. The language translation problem may yield piecemeal to the solution of good programs that "know" about very limited environment and "know" very little outside the environment. But this "very little" is substantially more than zero.

The second failure, TSS, exposed a problem: How to define and write very large software systems that were correct. Thereby it caused the births of a discipline of programming, of a methodology of programming management, and of a conservatism of system design and goal that have become the hallmarks of "soft ware engineering."

The chronology can barely intimate what has been occurring. An enormous and vital industry has been created that seems to make excellent use of the strengths of our society and seems unperturbed by our deficiencies.

Our society is characterized by mobility: social, economic, entrepreneurial and scientific. The computer flowers in such an environment, and nowhere has it flowered as much as in the United States. While controlled societies seem to have the greatest need to harness the computer, the device flowers best in environments of great intellectual mobility. So let us continue to view the computer not only as a tool for change, but as an instrument, unequalled outside the mind, on which to indulge our intellectual fantasies and to verify our humanity.

\section{References}

[1] David, E., "Some Thoughts About Production of Large Software Systems," NATO Conference Report on Soft ware Engineering (Garmisch. Germany, 1968).

[2] Newell, A. and Simon, H. A., "Computer Science as Empirical Inquiry: Symbols and Search," 1975 ACM Turing Award Lecture, Comm. ACM 19, 3 (Mar. 1976), 113-126.

[3] Gelernter, H., Sridharan, N. S., Hart, A. J., Yen, S. C., Fowler, F. W., and Shue, H. J., "The Discovery of Organic Synthetic Routes by Computer," Topics in Current Chemistry 1 (Springer-Verlag, 1973), 113-150.

[4] Newell, A., Perlis, A. J., and Simon, H. A., "What is Computer Science," Science 157, 3795 (Sept. 22, 1967), 1373-1374.

[5] Wick, J. D., Automatic Generation of Assemblers, Ph. D. thesis in computer science, Yale University, 1975. 



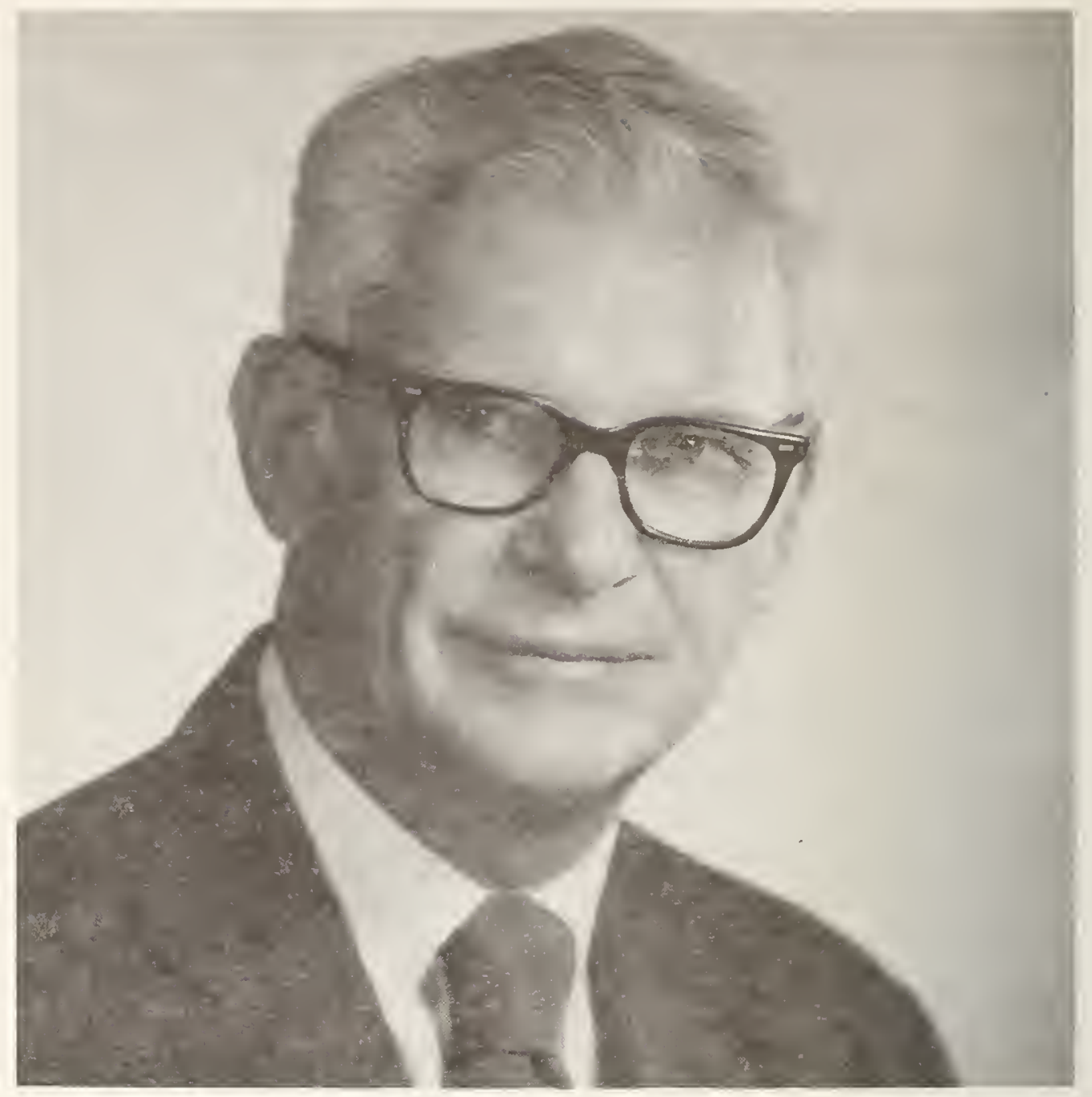

Dr. Robert Seamans is the Administrator of the new Energy Research and Development Administration. He has served as the President of the National Academy of Engineering, as the Secretary of the Air Force, and as Associate Administrator and Deputy Administrator of NASA. He was the Chief Engineer of the Missile Electronics and Control Division of RCA and held the Hunsaker Professorship at MIT. As an engineer, Dr. Seamans has had a distinguished research career at MIT in aeronautics, including instrumentation and control of aircraft amd missiles. He has received the Lawrence Sperry Award of the American Institute of Aeronautics and Astronautics, the NASA Distinguished Service Medal, the Goddard Trophy, the Department of Defense Distinguished Public Service Medal, and the Department of Air Force Exceptional Civilian Service Medal. 


\section{THE TECHNOLOGY OF ENERGY}

\section{America's Energy Background}

As the National Bureau of Standards celebrates three-quarters of a century of distinguished service to the nation, the United States is gearing its scientific, technological and managerial strengths to deal with the complex energy problems that confront us. The situation today is far different from that facing the $2,600,000$ inhabitants of the newly freed American nation. The energy resources available to our forebears were plentiful indeed, based predominantly on fuel wood from seemingly limitless forests backed up by falling water and by wind.

When the Centennial Year was celebrated in 1876, the population had grown to nearly 58 million and a new domestic energy resource had made its mark. It was coal. This black fossil fuel had increased its percentage of the energy consumed in the United States from a little over 9 percent in 1850 to around 50 percent. [1] Wood, water and wind-solar energy conversion-accounted for most of the rest. Petroleum, which had barely appeared by the time of the Centennial, accounted for less than 1 percent and natural gas for 1-1/2 percent. Despite the advent of these fuels, coal was rapidly becoming dominant and by $1901-$ the year the NBS was created-accounted for approximately 57 percent of energy consumed in the United States.

Its period of dominance, however, was limited. After peaking around 1910, coal, like wood before it, gave way to fast-rising petroleum and natural gas. Their relative cleanliness, their energy content, their low cost, and their convenience were so attractive that when the NBS celebrated its 50th anniversary in 1951 they had reduced coal's contribution to about 35 percent of the nation's energy "mix." Today, the figure is nearly 19 percent. Even though 1975 production was up over 1974's by about 5 percent, the black fossil fuel is now being extracted at levels similar to those of the 1940’s.

\section{The Modern Energy Resources Mix}

In the quarter century between NBS' 50 th and 75 th anniversaries, natural gas utilization increased from 18 to 28.4 percent and petroleum (including natural gas liquids) from 38 to 46 percent. Together, they supply about three-quarters of the energy consumed in the United States each year. This is graphically observed in figure 1. The 1975 share of natural 


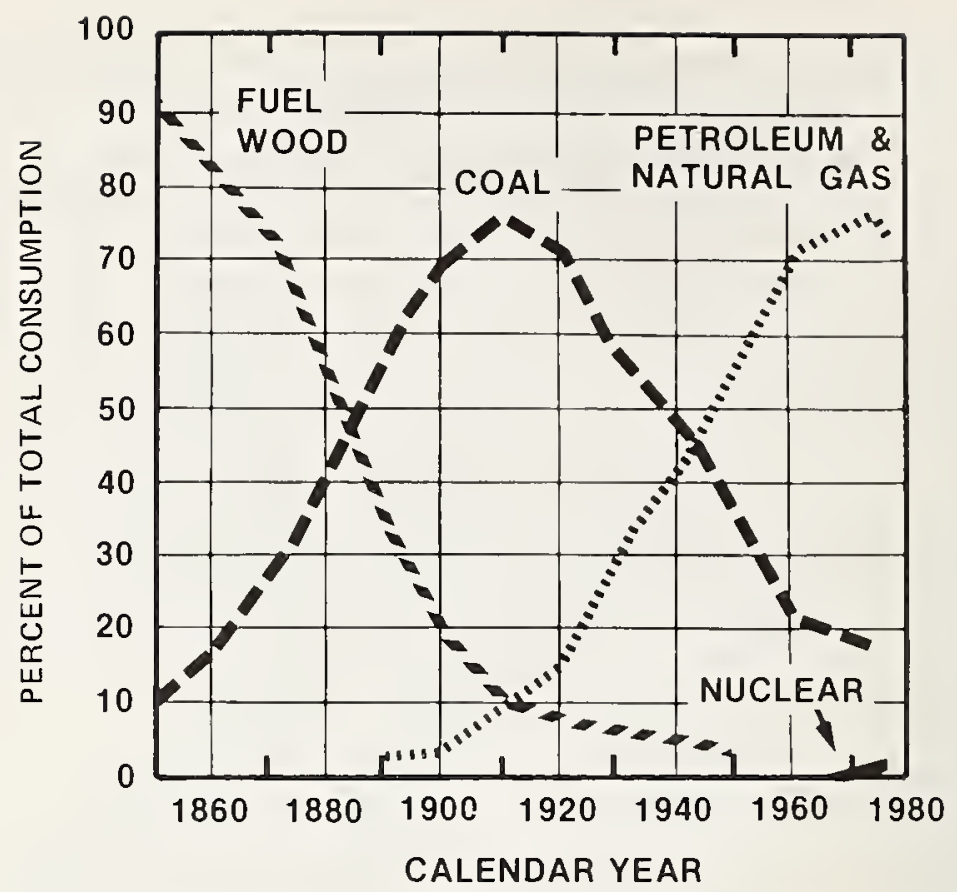

Figure 1. U.S. energy consumption patterns. Wood provided most of our energy in 1850 and 60 years later coal had become the principal source. By 1970, at the end of a second 60 year cycle, the Nation had become dependent on oil and natural gas for 75 percent of its energy. The next energy changeover cycle will have to be compressed. (Bureau of Mines)

energy consumption supplied by hydropower was 4.5 percent and nuclear 2.3 percent.

It is the clearly visible present-day dominance of oil and natural gas that is the cause of our energy crisis. Both are rapidly dwindling, irreplaceable resources. While we rely heavily on them for most of our energy needs, such abundant domestic resources as coal and uranium remain relatively underused.

In November 1970, U.S. domestic crude production reached its alltime peak of just over 10 million barrels a day. Since then, output has steadily dropped. During almost all of 1975 less than 8-1/2 million barrels a day were being produced. In December, the figure was 8.2 million barrels, and in March 1976 it dipped to less than 8.1 million barrels (fig. 2).

This declining production inevitably is reflected in the world arena. Back in 1938, before the Second World War broke out in Europe, we were producing 61 percent of world crude; in 1960 it was 33.5 percent; a decade later, 21 percent; and now it is less than 16 percent. Demand, meanwhile continues unabated.

The situation for natural gas is hardly better. Monthly production in the United States peaked at 1.98 trillion cubic feet in March 1973 and has been declining ever since - at a rate faster than oil. Reserves in the conterminous states stand today at levels of the early 1950's. Production during 


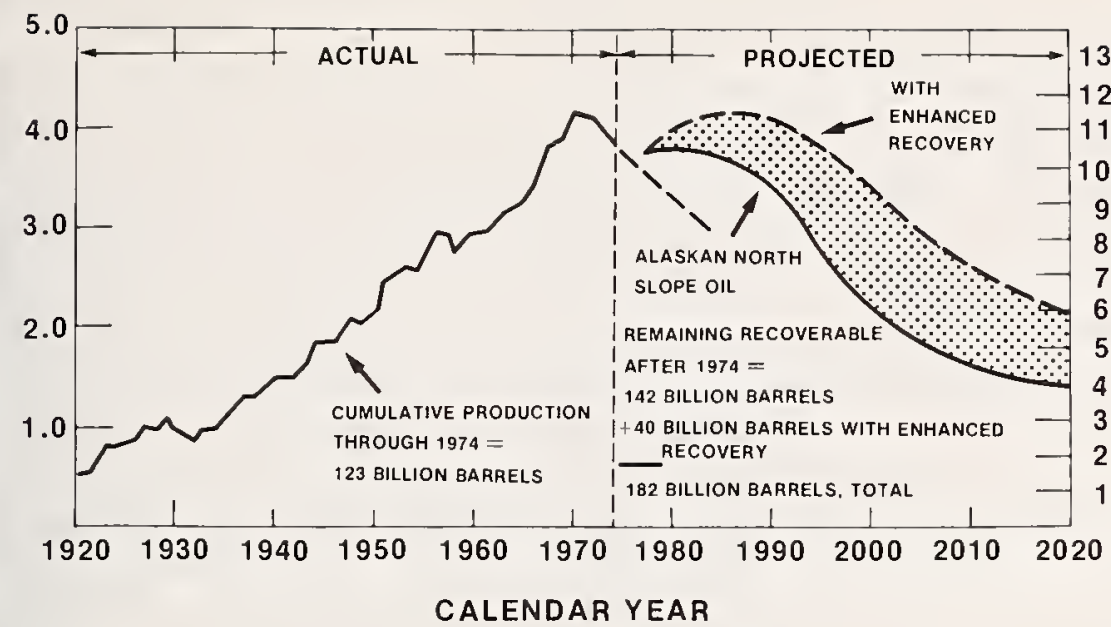

Figure 2. Projected U.S. oil production compared to production from 1920. Both crude oil and natural gas liquids are included. In 1975, for example, total U.S. crude oil production was 3.056 thousand million barrels to which were added 594 million barrels of NGL. Because of the downward slope of the curve, the United States is now importing 40 percent of its petroleum at an annual cost of some $\$ 34$ thousand million. (ERDA)

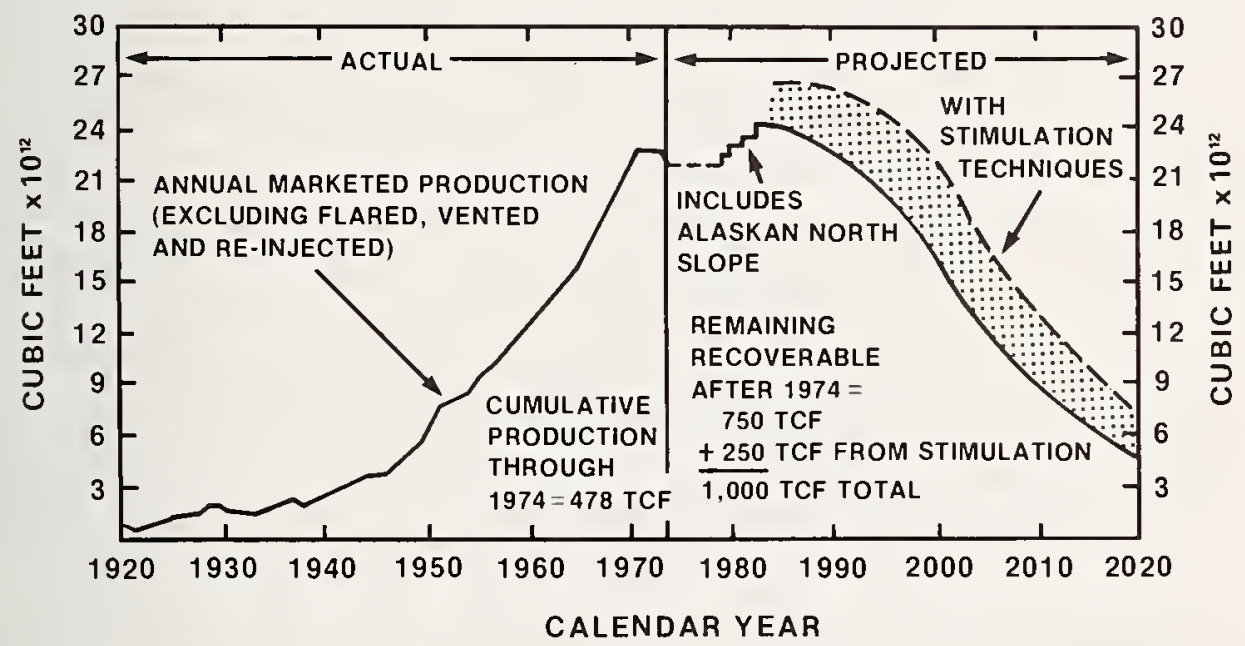

Figure 3. Past and projected natural gas production in the United States. Production from the Alaska North Slope will help gas (and oil) production for a while, but will not be too longlasting. Although gas production is now declining, consumption of this popular fuel increased 230 percent between 1950 and 1975. (ERDA)

the final quarter of last year averaged between 1.6 and 1.7 trillion cubic feet per month. The Bureau of Mines places overall 1975 production at 20.1 trillion cubic feet, down from 21.6 trillion cubic feet in 1974 (fig. 3 ). 
Even though there has been some slackening in overall energy demand since the winter 1973-74. Arab Oil Embargo, the gap between domestic production and domestic consumption of oil and natural gas has widened. This gap has been filled by imports, and today we are not only bringing to our shores more oil than we did in 1973 (during the week ending March 12, for the first time in our history, imports of crude oil and petroleum products exceeded domestic production), but a greater percentage of it comes from the very nations that participated in the embargo. This is partly because some customary suppliers other than those of the Organization of Petroleum Exporting Countries (OPEC) are cutting back on exports in the interest of conserving for future domestic needs. Canada, for example, last November announced a new export phase-out. From 750,000 barrels a day delivered to the United States in 1975, our neighbor to the north is scheduled to provide 460,000 barrels a day this year and 255,000 barrels a day next year. By 1980 , the figure is to drop to 45,000 barrels a day, then 10,000 in 1981, and nothing in 1982. Canada's traditional export of a trillion cubic feet of gas a year may also disappear by the early 1980's.

Regardless of the foreign source of the more than two out of every five barrels of oil consumed in the United States, imports are costly. As recently as 1970 , we paid about $\$ 3$ billion a year for imported oil. Last year, the bill climbed to $\$ 27$ billion, a ninefold increase. If it had not been for agricultural exports amounting to $\$ 22$ billion, the nation would have been in serious balance-of-payment difficulties. The import percentage is expected to increase through 1980, then decrease for 5 to 10 years as Alaskan oil makes its impact, then increase again.

By extracting over a hundred billion barrels of crude in a century or so of production, we have seriously depleted our domestic reserves. In fact, according to the latest U.S. Geological Survey study [2], only about 34 billion barrels of measured and 5 billion barrels of indicated economically-extractable reserves remain for a total of nearly 39 billion barrels. Another 23 billion barrels of inferred reserves may exist onshore and offshore. U.S. measured reserves compare with some 315 billion barrels in the Middle East and 49 billion in the Soviet Union.

As for natural gas, about 500 trillion cubic feet already have been used up, slightly more than the total remaining economically-recoverable measured (231 trillion cubic feet) and inferred (202 trillion cubic feet) reserves in our country. The reserve figures for the Sino-Soviet area are 736 trillion cubic feet and for the Middle East 594 trillion cubic feet.

Faced with these somber facts, the Director of the Geological Survey, Dr. V. E. McKelvey, commented that "...even if we are lucky in exploration, we will soon have to start shifting to other sources of energy as the mainstay of our supply." Making this shift is going to be no easy matter. But it must occur, for even Middle East reserves are finite. We are not likely to enjoy a leisurely phase-out of oil and gas as figure 1 illustrates we did when we moved away from reliance first on fuel wood and later on coal. 
As we begin to reduce our present exaggerated and unrealistic reliance on oil and natural gas, we should keep a number of facts in mind.

First, energy end-use patterns and the entire economic infrastructure are wedded to these two fossil fuels. The inertial resistance to changeover thus is going to be profound and time-consuming.

Second, this means that the United States will rely on oil and gas - domestic and imported-for a long time to come, even though efforts are augmented now to move away from them.

Third, in all likelihood, we will have to deal with the OPEC suppliers for an indefinite period. They control more than 65 percent of the oil in the non-Communist world, part of which keeps our nation moving.

Fourth, there is no short and easy road towards increased energy selfsufficiency. Only by exploring and eventually demonstrating relevant energy technologies and by developing a firm conservation ethic can we assure the future energy self-sufficiency of our nation.

Finally, we need not degrade our air and water environment as we shift away from oil and natural gas to multiple new energy options. With the aid of appropriate control technologies, we can enjoy a clean environment and adequate energy; they need not be mutually exclusive.

When we talk of energy self-sufficiency, we tend to think in terms of 5,10 or perhaps even 15 years. Such vision is necessary because of the long lead times in adapting new energy technologies to the marketplace and the requirements of future generations. Regardless of the lifestyle they elect to follow, our children are going to need energy, and so will their children. The decisions we make today will to a large extent determine how they live tomorrow.

In the past, the growth in the use of energy in our country has been impressive. A hundred years ago, the 58 million Americans then living used a little over 100 million Btu's per person. The 215 million of us now celebrating the Bicentennial Year are consuming nearly 340 million Btu's per capita, a more than threefold increase.

More significant than this, however, is what happened in recent years. During the decade preceding the Arab Oil Embargo, the annual rate of increase in U.S. energy demand was about 4.3 percent. This led to the United States with less than 6 percent of the Earth's population accounting for about a third of all energy consumed. Looked at from the per capita viewpoint, the average American today uses about six times more energy than the average inhabitant of the world as a whole.

Just as energy use in our country has grown in the past, so it is predicted to do so in the future. From 71 quadrillion Btu's consumed in 1975 , we may be using something like 82 quadrillion Btu's in 1980, 99 quadrillion Btu's in 1985, 115 quadrillion Btu's in 1990, and 156 quadrillion Btu's in 2000. 


\section{The Establishment of ERDA}

To guide the nation effectively towards a multiple-option energy mix in which such growth can be accommodated, the Energy Research and Development Administration (ERDA) was established on January 19, 1975. Although it was-and still is-a new agency, many of its components are not. In accordance with the Energy Reorganization Act of 1974 [3], the functions of the former Atomic Energy Commission except those of a regulatory nature; the energy research and development facilities, capabilities and personnel of the Department of Interior's Bureau of Mines; and all of the Office of Coal Research were turned over to ERDA.

The energy agency also absorbed the National Science Foundation's solar and geothermal programs and the alternative automotive power systems functions from the Environmental Protection Agency. These related elements and selective later recruitment have provided ERDA with a solid personnel and facility base from which to chart the nation's energy course.

Creating a new energy agency was one thing; charting a course the United States can follow towards increased energy independence is quite another. The Federal Nonnuclear Energy Research and Development Act of 1974 [4] required that ERDA develop a national energy program and then explain its rationale and the steps recommended to implement it.

\section{The National Energy Plan}

Accordingly, in late June of last year, ERDA's report was presented to the President and to the Congress covering a "National Plan for Energy Research, Development and Demonstration: Creating Energy Choices for the Future." [5]

One of its fundamental conclusions was that no single energy technology or even select groups of technologies could provide the flexibility and independence the nation requires.

To achieve such flexibility and independence, and to allow for some failures in the RD\&D process, the report emphasized that "multiple options [must be provided] which, taken all together, could exceed perceived needs." (See fig. 4.) The curtailment of any major existing option, such as the enhanced use of nuclear power or coal, would place such heavy and perhaps impossible demands on all remaining options that a significant reduction of crude oil and gas imports might not be achievable.

In revising the National Energy Plan this April, account was taken of various technical and scientific advances; trends in energy demand and production; new data on energy resources and reserves; regional, state and 


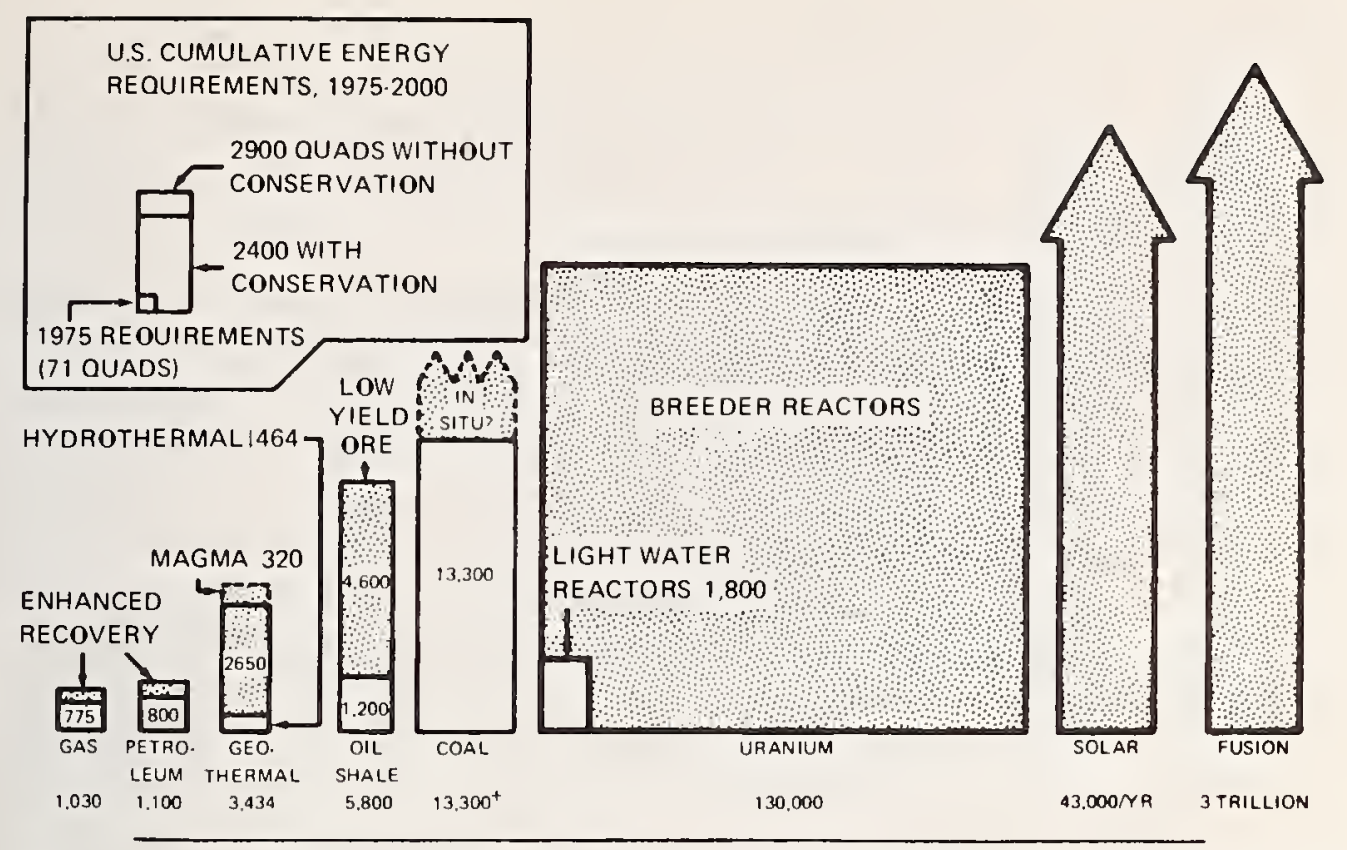

Figure 4. Known and potentially recoverable U.S. energy resources. The tiny square in the inset (upper left) gives a perspective on U.S. annual energy consumption measured in U.S. quadrillion (1015) BTU's or "quads." In 1947, energy consumption was 33 quads, in 1973 rose to nearly 75 quads, and in 1976 was about 74 quads. It is estimated that the cumulative energy requirements from 1975 to 2000 will be about 2,400 quads if an aggressive conservation program is carried out; otherwise, some 2,900 quads will be consumed. It is noted that petroleum and natural gas are our least plentiful resources yet we rely on them the most. Meanwhile, the United States has three times as much shale as oil and natural gas and six times as much coal. If we graduate from light water reactor to breeder reactor technology, some 60 times as much energy can be extracted from U.S. uranium reserves. Solar and fusion potential is essentially inexhaustible. Unshaded areas indicate energy available from resources using current technology. Shaded areas indicate potential energy contribution from new technology development. (ERDA)

local concern over energy developments and events; commercialization prospects for advanced energy technologies; institutional, regulatory and legal matters; international developments; and many other factors. Despite changing situations in all these areas, most of the principal conclusions of the original ERDA-48 Plan of June 1975 remain valid today.

Some shifting in emphasis is noted in ERDA-76 [6]-the popular reference for the revised Plan-notably the elevation of conservation technologies to the category having the highest priority for research, development and demonstration support by ERDA. By conservation, we mean the more efficient use of energy as well as the reduction of needless waste. 


\section{National Technology Goals}

To establish priorities in technology RD\&D and to develop strategies of implementation in the commercial sector, the Plan sets forth eight national energy technology goals:

* expand domestic supplies of economically recoverable, energyproducing raw materials, such as oil, gas, coal and uranium;

* over the longer term, increase the use of essentially inexhaustible domestic energy resources, for example, electrical conversion of solar energy, deuterium in the oceans using fusion power, and uranium 238 with breeder technology;

* transform such abundant fuel resources as coal into more desirable end-use forms, that is, into clean-burning liquid and gas substitutes;

* augment the efficiency and reliability of the processes used in energy conversion and delivery systems-improved converter-type nuclear power reactors, for example, and better electric power transmission and distribution;

* change consumption patterns to improve energy use, an example being the introduction of solar heating and cooling technology into the residential and commercial building markets;

* increase the efficiency of end-users, such as improved automotive vehicles, appliances, and industrial equipment;

* perform basic and supporting research and technical services directly and indirectly related to energy; typically, these include exploration and resource assessment, nuclear fuel cycle support, and fossil fuel transportation such as coal slurry lines;

* protect and enhance the general health, safety, welfare and environment during the course of energy development and systems implementation; the introduction of environmental control technologies and the establishment of standards of environmental performance of each technology concept are included in this area.

\section{Strategic Approach to Energy RD\&D}

As the National Energy Plan evolved, various research, development and demonstration programs were recommended to implement these priorities, all with the aim of facilitating the changeover from a diminishing base of oil and natural gas resources to a broad range of alternative-and much less limited-resources.

The exploitation of these resources will require the development of many new energy technologies. Since the private sector is the main producer and consumer of energy, it is going to be up to it - and not the 
government - to assume the principal role of moving these technologies from early development through the demonstration phase and into the marketplace. The role of ERDA, and of other Federal agencies involved in energy, is and must remain supplementary.

Ultimately, the actions of private enterprise and of the market forces that shape them will change the energy mix of our nation. Whereas fossil fuels - coal, oil and gas - now supply nearly 94 percent of the overall energy requirements of our country, by the year 2000 they may only provide between 65 and 70 percent. Nuclear's contribution is expected to rise from the current 2.3 percent to between 25 and 30 percent; hydropower and geothermal systems may account for approximately 4 percent. Solar heat ing and cooling should contribute an additional few percent.

The principal recoverable resources of the United States (in thermal equivalents) that are likely to provide most of the energy out to the end of the century are summarized in table l. ERDA-76 (the revised Plan) estimates in column 6 are compared with those of the earlier ERDA-48 in column 2. The new assessments are based on different interpretations of then-available data and - in coal's case- on an upward revision of reserve estimates.

\section{First Strategic Element}

Although the first strategic element of both the original and revised versions of the National Energy Plan addresses U.S. needs during the next decade, ERDA-76 added a 5-year planning projection which, it is contemplated, will roll forward every year. This short-range look ahead will focus principally on conservation and will continually be monitored for successes and for failures.

\section{Conservation Techniques}

Recognizing that even a decade is inadequate for the development of whole new energy systems, ERDA-76-as we have already noted-assigned top national priority to those conservation technologies which act immediately to reduce energy consumption. Such technologies will be applied towards creating more energy-efficient buildings, consumer products, industrial processes, and individual and public transportation systems. Among the reasons for the heightened emphasis on conservation are:

* Each barrel of oil saved is one less that needs to be imported. Conservation, combined with programs leading towards fuel substitution, act to reduce dependence on foreign oil. 


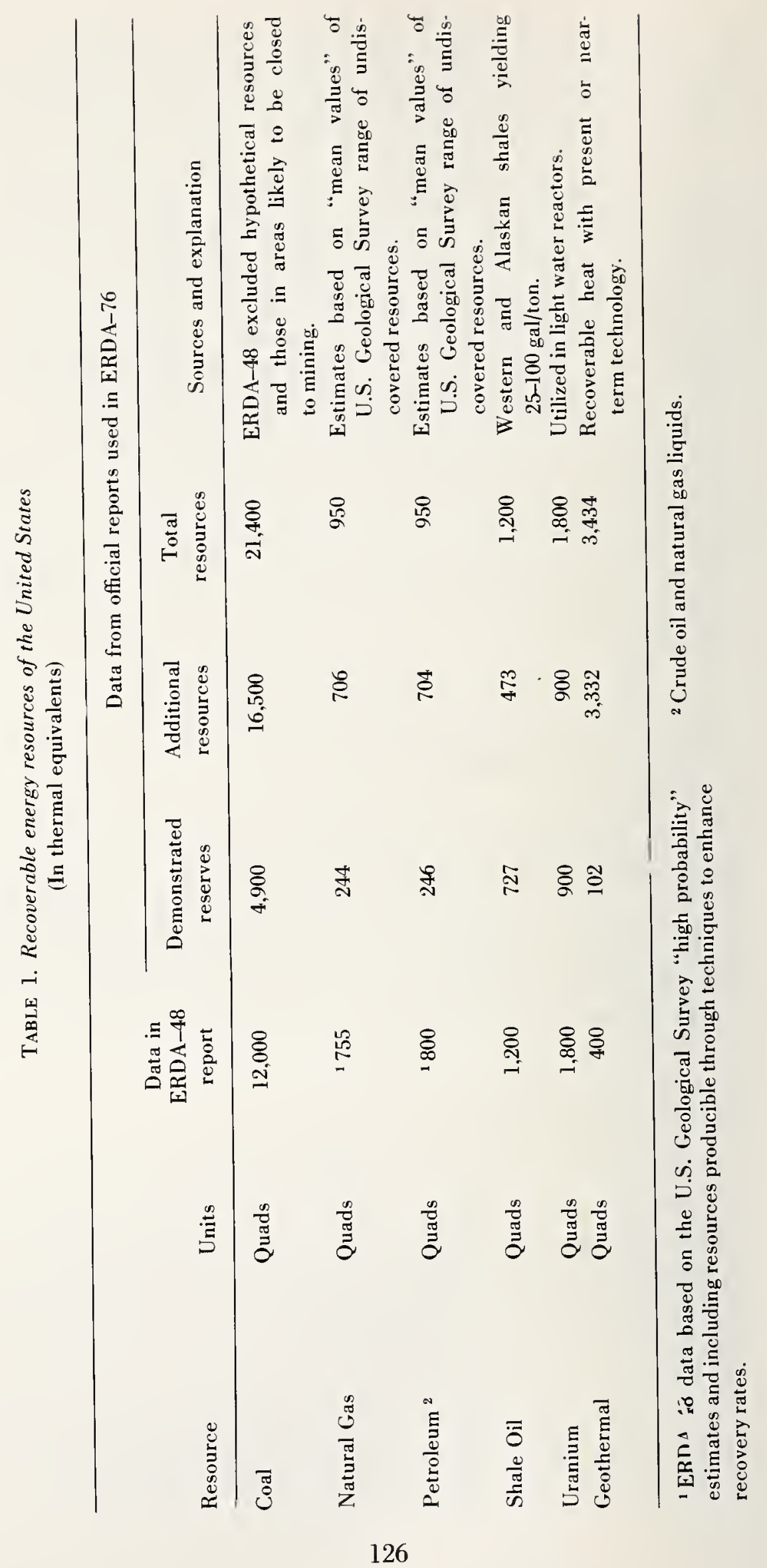


* It generally costs less to save a barrel of oil than to produce or import one.

* Energy conserved is environmentally beneficial.

* Capital needed to increase energy-use efficiency is normally less than that involved in producing an equivalent amount of energy; this is because new energy supply technologies are highly capital-intensive.

* The near-term use of existing technologies can generally be ap plied by the private sector with a lessened need for government assistance.

* Conservation also helps to preserve for future generations the limited legacy of fossil fuels and uranium left to us by nature.

Backing up conservation are parallel near-term programs to convert waste materials to energy and to utilize, on a broadened scale, conventional domestic energy resources. Efforts in the latter area are directed (1) towards the enhanced recovery of oil and natural gas by fluid injection, massive hydraulic fracturing and other techniques in identified fields, and (2) the discovery of new fields, offshore as well as on.

\section{Light Water Reactor Program}

Equally important to the U.S. energy picture during the coming decade and beyond is the increased use and improvement of light water nuclear reactors for electric power generation. As of January 1976, a total of 58 nuclear electric generation units was operating in the United States with a capacity of $39,595 \mathrm{MWe}$. Construction permits have been issued for another 69 units totaling 70,773 MWe and limited work authorizations have been released for 18 more with a capacity of 17,364 MWe. In addition, there are 72 units on order as of the first of the year. They are expected to add $82,835 \mathrm{MW}$ to the nation's overall nuclear electric capacity [7].

Considerable concern has been expressed as to the safety of nuclear reactors, yet during over 1,700 reactor-years of commercial and lightwater military reactor experience no melt-down or other potentially serious nuclear-related accident has occurred. So safe has nuclear power been, in fact, that in trying to determine the possibility of accidents one must use theoretical predictions rather than actuarial tables.

Commercial nuclear power's excellent safety record has not diminished efforts by the Nuclear Regulatory Commission, by ERDA and by industry to insure continued safe operations. Typical of the activities now under way is the advanced safety system design program which seeks to identify alternative designs for lower cost yet highly reliable safety systems for emergency cooling, containment and core coolability.

Concern has also arisen as to nuclear fuel supplies. According to ERDA studies, the nation's identified uranium resources are adequate to 
supply the lifetime fuel requirements of reactors now operating, under construction or planned, as well as those of additional reactors that may come on line in the near- and mid-terms. ERDA's National Uranium Resource Evaluation (NURE) program puts U.S. resources of $\mathrm{U}_{3} \mathrm{O}_{8}$ recoverable at a production cost of $\$ 30$ or less a pound at 3.56 million tons. Of these, 640,000 tons are known reserves and 2.92 million tons are potential resources [8].

Efforts are now being stepped up within NURE to locate new supplies of uranium ore in our country. But, as important as new ore discoveries are, we need to increase simultaneously our all-too-limited enrichment capability. In recognition of this fact, the President late in February again urged Congress "to give high priority to my Nuclear Fuel Assurance Act to provide enriched uranium needed for commercial nuclear power plants here and abroad." This act, first recommended to Congress in June 1975, would permit ERDA to negotiate and to enter into cooperative agreements with the private sector enabling it to commercialize enrichment operations. Such an approach not only is consistent with the overall policy of moving energy technologies into the marketplace as soon as practical, but promises to close an obvious and serious shortcoming in the fuel cycle.

Industry has proposed four enrichment projects that it is willing and able to undertake. These are based on the conventional gaseous diffusion approach as well as the newer centrifuge method. ERDA, meanwhile, is examining still another and potentially more economic uranium enrichment concept involving laser isotope separation.

With the prospects improving for increased industrial participation at the "front end" of the fuel cycle, ERDA will be able to concentrate more heavily on filling in gaps at the "back end." This involves the demonstration of technology related to spent-fuel reprocessing, the recycling of recovered uranium and plutonium, and the development of long-term disposal techniques for radioactive wastes produced by commercial reactors. Progress in these areas eventually should permit the private sector to assume responsibility for both the front end and the back end of the nuclear fuel cycle - under appropriate controls, of course.

In recent years, the public has voiced its concern over the processing, the handling, and particularly the disposal of radioactive wastes. These include highly penetrating fission fragments and traces of plutonium and similar elements collectively termed transuranium elements. From the handling standpoint, the most difficult wastes are the fission fragments. Yet, the volume that would accumulate during the rest of this century from a thousand commercial nuclear power plants would occupy a space of only about 100 square feet. In practice, of course, such wastes would not be stored in one place but rather in multiple containers probably in terminal storage sites within stable geologic formations.

For over three decades ways and means of protecting the biosphere and underground waters from these and transuranium wastes have been 
studied and developed. The stable geologic formation approach seems so attractive that this year we plan to demonstrate a site in a deep-bedded New Mexico salt deposit.

In view of political instability in many parts of the world and continuing acts of terrorism, the possibility is not necessarily remote that terrorists or other groups might be tempted to divert nuclear materials. Substantial increases in that portion of ERDA's budget devoted to nuclear controls and safeguards reflect this possibility.

The major thrust of ERDA's safeguards program is to assure the safety of all light water reactor fuel cycle facilities in our country. To help accomplish this, computer models have been developed that allow us to measure and evaluate safety and control systems against a wide range of potential accident and threat situations. We are also developing new technologies that account for as well as physically protect nuclear materials on site and while being transported.

Around fixed installations, for example, armed guards are supplemented by multiple physical barriers equipped with alarm devices. Strict control is maintained over facilities and operations at all times. Thus, critical areas are continuously monitored by television, and multiple communication systems are used in close cooperation with local law enforcement agencies. Also, all principal security control facilities are hardened against possible armed attack. When nuclear materials are shipped from one site to another, armed guards and special armored vehicles are employed. Each shipment is traced at a control center which is in frequent radio or telephone communication with the transporting vehicle.

The adequacy of uranium reserves is but one factor favoring the increased use of light water reactors for electricity generation in our country. Others include price, with nuclear holding a clear edge over competitive coal-fired systems in the northeast and an apparent cost advantage for the Atlantic coast, the southeast, and the inland Great Lakes region. These areas are expected to require about three-fifths of the added electric power generating capacity out to the end of this century. By way of comparison, coal has an apparent cost advantage in the mid-continent and Rocky Mountain regions, while a stand-off exists for the Pacific coast.

As far as the average costs of electric power from various fuel sources is concerned, during 1975 nuclear was a little under 13 mills per kilowatt hour, coal somewhat over 17 mills per kilowatt hour, and oil more than 33 mills per kilowatt hour. But this is the direct consumer-related part of the story. It has been estimated that because of the use of nuclear power in the United States during 1975 around 300 million barrels of oil were conserved, many of which would have been purchased from abroad. 
The expanded exploitation of America's vast coal resources is also contemplated for the 1985-2000 mid-term: Coal now remaining in the ground, according to a U.S. Geological Survey study published last year [9], totals an estimated 3,968 billion short tons, a 23 percent increase over previous estimates. Of these, 424 billion tons constitute the identified reserve base and 1,307 billion tons, additional identified resources. About 5 percent of our total reserves - some 200 billion tons - is believed to be extractable using conventional technology. This alone is five times the estimated cumulative production of coal in the United States.

In exploiting this coal, emphasis is being placed on fluidized-bed combustion for industrial process heat and some utilities. This process of burning coal removes more than 90 percent of the emitted sulfur dioxide and reduces nitrogen oxide and particulate matter to levels under those established by the Environmental Protection Agency for new coal-burning installations. Improvements in stack gas cleaning and nitrogen oxide control systems are expected to make the use of coal increasingly acceptable from the environmental point of view.

\section{Second Strategic Element}

Our analyses reveal that unless immediate action is taken, a serious liquid and gaseous fuels "gap" is likely to appear in the mid-term period. By then Alaskan oil production is expected to have peaked and begun to drop off while that in the conterminous states continues its inexorable decline. Preventing the gap from occurring, then, forms the basis of the National Energy RD\&D Plan's second strategic element.

Several approaches to reducing liquid and gaseous fuel consumption appear necessary to ward off the looming mid-term crisis. One calls for the continued development of end-use conservation technologies. Another involves increased reliance on geothermal, solar heating and cooling, and other under-used resources and on the extraction of energy from waste heat. Concurrent with these efforts are others to enhance oil and gas recovery from domestic fields and to derive synthetic substitutes from coal, oil shale and tar sands.

\section{Geothermal Resources}

It is estimated that by the beginning of the mid-term, 0.8 quads per year may be produced from geothermal resources found in many regions of the United States. This contribution could well grow to 4.4 quads/year by the end of the century, and 18.6 quads/year by 2020 [10]. Geothermal 
resources are defined as identified and still-to-be discovered stored heat that can be recovered and used with technology that is or soon may become available. Five basic types of such resources have been identified: (1) hydrothermal convective, (2) hot dry rock structures; (3) so-called normal gradients which are conduction-dominated areas within the first 10 kilometers of the crust; (4) high-temperature molten magma; and (5) geopressured water reservoirs [11]. Table 2 indicates those resources that can be tapped with near-term technology.

In early April of this year, ERDA requested proposals for geothermal R\&D in the following areas: (1) engineering and economic studies on nonelectric applications of geothermal heat; (2) improving and developing well logging tools, instruments, and materials; (3) conducting field tests of stimulation methods with the aim of improving and prolonging geothermal well productivity; and (4) testing of wells in geopressured aquifers.

TABLE 2. Geothermal resources-estimated recoverable heat with present or near-term technology ${ }^{1}$

\begin{tabular}{|c|c|c|c|}
\hline \multirow[b]{2}{*}{ Resource type } & \multicolumn{3}{|c|}{ Quads (quadrillion BTU) } \\
\hline & Known & Inferred & Total \\
\hline \multicolumn{4}{|l|}{ Hydrothermal convection } \\
\hline Vapor dominated $\left(>150^{\circ} \mathrm{C}\right)$ & 2 & 2 & 4 \\
\hline \multicolumn{4}{|l|}{ Liquid dominated } \\
\hline High temperature $\left(>150^{\circ} \mathrm{C}\right)$ & 20 & 110 & 130 \\
\hline Low temperature $\left(90-150^{\circ} \mathrm{C}\right)$ & 80 & 250 & 330 \\
\hline \multicolumn{4}{|l|}{ Geopressured } \\
\hline Electrical utilization & 100 & 230 & 330 \\
\hline Methane production & 500 & 1,500 & 2,000 \\
\hline Hot dry rock structures & 80 & 240 & 320 \\
\hline Magma & 80 & 240 & 320 \\
\hline Total & $862^{2}$ & $2,572^{2}$ & $3,434^{2}$ \\
\hline
\end{tabular}

${ }^{1}$ Does not include (1) normal gradients of heat in the earth, nor (2) hydrothermal convection systems of less than $90^{\circ} \mathrm{C}$ temperature.

${ }^{2}$ These figures should be rounded off to $900,2,500$ and 3,500 , respectively, to indicate that they are rough estimates based on many uncertainties, most particularly with respect to magma resources. Since these are presumably renewed from deep within the earth, they may in fact be much more significant than indicated in this table. 
Solar heating and cooling technology is expected to advance briskly and by the mid-term could be making a significant impact, particularly in the residential sector. The Federal part of the National Program for Solar Heating and Cooling includes (1) demonstrations of hot water heating and space heating as well as combined heating and cooling for residential and commercial applications; (2) development in support of the demonstrations; and (3) research and advanced systems development in solar heating and cooling technology $[12,13]$.

Progress has already been substantial. The first large-scale demonstration of solar energy in residential housing was announced in mid-January of this year by the Department of Housing and Urban Development with the awarding of $\$ 1$ million in ERDA pass-through grants for the installation of 143 units in 27 states. Then in February, ERDA selected 36 complete systems for solar heating, water heating, or heating and cooling of residential and commercial buildings as technically acceptable for use in the demonstration program. Still more recently, in early April, ERDA announced the selection of 34 nonresidential buildings in 22 states and the Virgin Islands for the installation of solar heating and/or cooling systems.

Among the many tasks facing those involved with the national solar heating and cooling program is the need to identify factors that restrain the widespread utilization of solar systems and to seek ways of removing or at least mitigating them. Constraints range from the lack of trained personnel to install, start-up and operate new and unfamiliar solar heating and cooling systems to the reluctance of financial institutions to provide mortgages to homes incorporating them. Such reluctance is understandable for we are dealing with a new technology and an industry that is still in its infancy.

As the solar industry develops, as objective standards evolve, and as information is more widely disseminated, developers, builders and customers alike may begin to understand that savings in operating costs over the lifetime of an individual housing investment may more than offset the higher-than-normal initial installation cost of solar-based systems. It is interesting to note that several states have enacted laws to provide property tax exemption for individuals, commercial entities, and institutions that install solar water heating and space heating and cooling in their structures.

The utilization of waste heat may also become important in the midterm, helping thereby to reduce demand for liquid and gaseous fossil fuels. Today, our society discards large quantities of low-grade heat into the at mosphere at points of generation (powerplant sites, factories, homes). Ways of utilizing the energy content of this residual heat must certainly be explored. 
Probably the most important mid-term approach is directed at new ways of obtaining oil and gas from domestic resources. For institutional and other reasons, their use will almost certainly persist for as long as they are economically available to the public. The question is, where are they going to come from?

\section{Enhanced Oil and Gas Recovery}

Near-term enhanced crude oil and natural gas recovery techniques are certainly going to be vigorously pursued, on-shore and off in the "lower 48", and in Alaska. It is estimated that up to 40 to 60 billion barrels of crude oil may become economically producible through the application of advanced (tertiary) recovery methods. The resource base in which this oil is being searched consists of an estimated 290 billion barrels of normal-gravity oil, 107 billion barrels of heavy oil, and 30 billion barrels of bitumen [14]. These figures compare with measured reserves of a little over 34 billion barrels and indicated reserves somewhat under 5 billion barrels. As for natural gas, a resource base of some 600 trillion cubic feet may exist, which is considerably larger than the 237 trillion cubic feet of measured reserves in our country.

Among the approaches being investigated to extract hitherto unreachable oil are fluid injection and thermal and chemical techniques. Requests for proposals were released in late December of 1975 for cooperative ERDA-industry field demonstration projects using a micellar-polymer chemical flooding process. Detergent-like micellar chemicals are used to release the oil following which polymer-thickened water is employed to control and direct the micellar chemical flow. Plain water is then used to force the oil-chemical mixture to the well. In February 1976, similar requests for proposals were made for projects using improved waterflood techniques. ERDA's aim is 500,000 barrels of oil per day of increased production.

A number of projects are under way to stimulate gas production in tight formations through the use of fracturing techniques. One of these is massive hydraulic fracturing and another is chemical explosive fracturing. In both cases, the idea is to induce fractures and fissures in tight geologic formations, exposing thereby the gas resources to the well bore. ERDA hopes to use such techniques in meeting its 1985 goal of 3 billion cubic feet a day of enhanced recovery of gas.

\section{Synthetic Fuels}

In addition to enhancing the recovery of naturally-occurring oil and gas, technologies are available for demonstrating the economical and en- 
vironmentally acceptable extraction of oil and gas substitutes from coal, oil shale and tar sands within the President's Synthetic Fuels Commercialization Program [15]. Synthetic fuels, popularly known as synfuels, are already being produced on an experimental basis in the United States and programs for scaling up to demonstration plants are actively under way at ERDA. Among the elements of the program are (1) the liquefaction of coal to yield boiler fuels and crude oil substitutes for refinement, and (2) the gasification of coal to yield low-Btu fuel gas for electricity generation and for industrial processes and high-Btu pipeline-quality gas for residential and commercial uses.

In the oil shale area, industry participation is being sought to demonstrate the feasibility of recovering oil by in situ (removing part of the shale by mining) retorting methods and to determine the best fracture or explosive rubblizing technique for the resources involved. Projects to demonstrate ways of recovering oil from tar sands are also being developed.

Assuming that appropriate legislative, budgetary and administrative actions are taken soon, the production of synfuels could reach a million barrels a day of oil equivalent by 1985. A full-fledged industry could be built up during the following 10 years or so, leading to the production of at least 5 million barrels a day by the mid to late 1990's. Typically, a commercial size plant would produce 50,000 barrels of oil a day or 250 million cubic feet of gas a day.

\section{Third Strategic Element}

The third strategic element of the National Energy Plan is concerned primarily with the needs of our descendents living in the 21st century, though efforts taken on their behalf may contribute to the U.S. energy picture by the 1990's. This element seeks ways to exploit what are essentially inexhaustible energy resources-energy from deuterium found in the world's oceans, energy from uranium-238, and energy from the Sun. The technological development of these resources hopefully will lead to successful breeder reactor, solar power, and fusion reactor systems.

\section{Breeder Reactor}

The first of these, the breeder reactor, offers the long-term prospect of unlocking the energy potential of uranium-238 (and of thorium-232), and of converting it to electricity and perhaps to some extent to industrial process heat. Whereas conventional light water reactors generate energy almost completely through the fission of U-235, breeders are able to utilize U-238 which constitutes the overwhelming percentage of the uranium brought out of mines. The breeder concept is based on the fact that through neutron 
bombardment essentially nonfissionable U-238 can be converted to fissionable isotopes of plutonium and, in addition, nonfissionable thorium-232 can be converted to fissionable U-233.

The breeder, which is attractive because it promises to increase the energy value of domestic uranium reserves 60 or more times, has proven to be a technologically sound concept. Indeed, eight breeders are in operation in various parts of the world at this time, including three in the U.S.S.R., two each in France and the United Kingdom, and one in the United States. The American entry is the Experimental Breeder Reactor No. 2, which is currently being used for fast flux irradiation testing of fuel and structural materials. It operates at full power with a plant availability of between 60 and 65 percent.

The Liquid Metal Fast Breeder Reactor at Clinch River in Tennessee [16] represents this country's principal effort to demonstrate the commercial feasibility of the breeder concept. The program is aimed at developing a broad technological and engineering base from which a competitive commercial breeder industry can emerge, hopefully sometime beginning in the 1990 's. It also seeks to demonstrate to the utility industry and to the public that in addition to being economical, the breeder is a safe and environmentally acceptable energy source [17]. Utilities and industries have been closely involved with the LMFBR program since its inception.

To fulfill LMFBR objectives, seven principal areas requiring rigorous effort have been identified. First of all, the reactor core is being redesigned with the aim of improving performance and plant economics. Secondly, mechanized and automated fuel production methods are being developed so that fuel element fabrication costs can be lowered. In this context, advanced fuels are being investigated that will not only generate more power in the core at lower costs but will permit the breeding of new fuels at faster rates. Other efforts include improving the coolant system, components, and fuel processing and recycling; developing new construction materials; and studying and addressing safety and environmental aspects of breeder design and operation. The projected start-up date for Clinch River operations is 1983. By 1986, sufficient operational information should be accumulated so that an ERDA decision regarding LMFBR's commercialization can be made.

\section{Solar Electric Power}

The second long-term energy option open to the United States involves the generation of power from the Sun. Based on estimates of the total solar energy incident on this country during an average year, and postulating a 10 percent collector efficiency, a mere 4 percent of this energy could theoretically meet all projected U.S. requirements at the end of the century. 
Four technologies are being developed to capt ure the Sun's energy: (1) solar thermal electric (which can also supply industrial process heat and space heating); (2) solar voltaic conversion; (3) wind energy conversion; and (4) ocean thermal conversion based on temperature differentials occurring between surface and deep-water layers [12]. (Other renewable ocean resource options include waves, tides, salinity gradients, and currents; all are being investigated.)

The greatest potential appears to lie in ERDA's solar thermal activities. These involve systems that collect solar radiation and convert it to thermal energy. Subsequently, the heat is transferred to a working fluid for use in generating electricity; or, alternatively, the heat is transferred to a total energy system to provide both electricity and thermal energy.

Two types of such systems are being considered at the present time: (1) a central receiver where a large array of Sun-tracking mirrors or heliostats concentrates solar radiation on a central thermal collector (boiler), and (2) a distributed thermal collector that uses a large array of small concentrating systems to collect the radiation. Steam produced in the boiler converts the Sun's energy into electric power through a turbine and generator.

ERDA is developing a program whereby it plans to seek proposals for the location and operation of the nation's first solar thermal electric pilot plant of the central receiver type. Construction is to begin in 1978 with completion set for late 1980 . When ready, the plant is to have a 10 megawatt electrical generating capacity and is to be integrated into an existing utility network.

\section{Fusion Reactor Technology}

ERDA is pursuing a long-term program to harness the energy resulting from the fusion of the nuclei of deuterium with tritium, both of which are isotopes of hydrogen. The former is available in virtually unlimited quantities in the world's oceans, while the latter is obtained from lithium found both in water and in the Earth's crust. To permit fusion to occur, light elemental nuclei in the form of a plasma must be confined at high densities and temperatures $(\sim 100,000,000$ degrees $C)$.

Two parallel fusion approaches are being investigated by ERDA, one involving the magnetic field confinement of fusion fuel and the other involving inertial confinement by means of laser or electron-beam energy.

The principal magnetic systems-tokamak (a Russian word for toroidal magnetic chamber), theta pinch, and magnetic mirror-all require a combination of high temperatures, dense plasmas, and long confinement times to permit a sufficient number of fusion reactions to take place [18]. The tokamak is a low-density closed system receiving about 65 percent of 
ERDA's emphasis, the theta pinch is a high-density system with 15 percent, and the magnetic mirror is an open system also with 15 percent. Another 5 percent is reserved for exploratory concepts.

The Oak Ridge National Laboratory's ORMAK device has demonstrated that magnetic confinement improves as the square of the plasma size, and has further demonstrated plasma heating up to $15,000,000{ }^{\circ} \mathrm{C}$. More recently, in December of last year, the Princeton Large Torus entered into operation. It is the first experimental device in this country large enough to produce conditions approaching those required for fusion reactors. Beyond the PLT a still larger tokamak is scheduled to be built and should be capable of burning deuterium and tritium fuel on a sustained basis. It has been designated the Tokamak Fusion Test Reactor. Other devices being constructed for the tokamak program are: the Poloidal Diverter Experiment, Doublet III, and the Impurity Study Experiment.

In March of this year it was announced that the Lawrence Livermore Laboratory's 2X-IIB experimental magnetic mirror device achieved a factor of two increase in the product of fuel density and temperature over what had earlier been considered theoretical limits. The magnetic mirror receives its name from the fact that strong magnetic fields are used at both ends of the device to reflect or mirror energetic fuel particles back into the system. The $2 \mathrm{X}$-IIB is the major experiment in the magnetic mirror program; others are Baseball II and LITE. Scyllac, the principal device employed in the theta pinch effort, has achieved "ignition" temperatures of $50,000,000^{\circ} \mathrm{C}$.

Magnetic fusion program milestones are: (1) production and understanding of reactor-level hydrogen plasmas in 1978-1980; (2) production of substantial quantities of thermal energy in the tokamak fusion test reactor in 1982-1985; (3) production of substantial quantities of electrical energy in experimental power reactors in the mid 1980's, and (4) the operation of a commercial scale demonstration power reactor by the late 1990's.

The laser fusion concepts under investigation at ERDA rely on the inertia of material which is made to implode towards the center of a spherical geometry. This results in the compression of deuterium-tritium fuel pellets to densities on the order of a thousand times the density of normal solids and in heating to "ignition" the thermonuclear reaction. Energy is released in the form of $\mathrm{x}$ rays and high-speed atomic particles which are converted to heat by absorption in the surrounding chamber. Laser fusion plants are expected to use their heat to generate steam for electricity and perhaps for chemical processes. High-energy electron beams can also be focused on fuel pellets, providing a potentially viable alternative to laser-driven fusion. A commercial-scale inertial confinement demonstration fusion power plant is projected for the mid-1990's. 
Among the many advantages inherent in fusion reactor technology are:

* Virtually infinite fuel supply available at extremely low cost.

* Safety; no possibility of nuclear runaway.

* No chemical combustion products involved, and no after heat cooling problems associated with accidental coolant loss.

* Relatively low radioactivity and attendant hazards.

* As weapons-grade nuclear materials are not employed, concern over diversion for terrorist or blackmail purposes does not arise.

* Considerable siting flexibility, permitting fusion power plants to be located near centers that will utilize their energy output.

Complementary to the National Energy Plan's third strategic approach is the development of technologies that will be needed to accommodate the distribution of the essentially unlimited sources of energy just reviewed. It is expected that this energy will appear primarily as electricity. Hydrogen may also assume an important role as an alternate fuel in the transportation and other sectors of our society. The conversion of biologically degradable materials ("biomass") to such fuels as alcohol or methane gas may evolve into still another important energy technology for the long. term.

\section{RD\&D Priorities}

To accomplish near-, mid- and long-term energy goals, national RD\&D priorities have been tentatively established. Only by setting such priorities can resources be allocated effectively to the RD\&D process with the reasonable expectation that a given energy technology will be ready when it is needed.

Priorities, like all else in the National Energy Plan, are - and of necessity must be-flexible. For example, the development of electrification and hydrogen fuel technologies has been given a relatively low priority today. Their positions, however, are apt to rise as confidence in breeder, solar electric, and/or fusion power systems increases in a decade or so.

Table 3 ranks the many RD\&D technologies in which ERDA is involved [6]. Regardless of the level now assigned the various energy technologies, all to a greater or lesser extent must be - and indeed are being-pursued. There are a number of compelling reasons for this.

The research, development and demonstration of one or more technologies may fail or become seriously delayed, making it prudent that back-up options be available at all times. Then, too, the long-term approaches have not yet been proven to be technologically, much less commercially, feasible, and will require an extended period of development.

Furthermore, in order to make the importing of significant quantities of foreign energy a matter of choice rather than of necessity, a broad range 


\section{HIGHEST PRIORITY DEMAND}

Near-term efficiency (conservation technologies)
- Conservation in buildings \& consumer products

- Industrial energy efficiency

- Transportation efficiency

- Waste materials to energy

\section{HIGHEST PRIORITY SUPPLY}

Major near-term energy systems

New sources of liquids and gases for the mid-term

"Inexhaustible" sources for the long-term
- Coal-direct utilization in industry and utilities

- Nuclear-converter reactors

- Oil and gas-enhanced recovery

- Gaseous and liquid fuels from coal

- Oil shale and tar sands

- Breeder reactors

- Solar electric systems

- Fusion systems

\section{IMPORTANT TECHNOLOGIES}

Under-used mid-term technologies

Technologies supporting intensive electrification
- Geothermal

Solar heating and cooling

Waste utilization

- Electric conversion efficiency

- Electric power transmission and distribution

- Electric transport

- Energy storage

- Fuels from biomass

- Hydrogen in energy systems

of new technologies must be introduced at the earliest possible time. Still another reason for avoiding new technology start-up delays is the possibility that some seemingly attractive energy systems may not be fully - or even partially-exercised because of environmental, economic, social or other essentially nontechnological restraints.

It behooves us not to underestimate these restraints as the nation moves from limited to less limited and eventually almost unlimited alternative energy resources. Many environmental, social, institutional, regulatory and other realities must be faced and public acceptance gained. The latter is vitally important, for it is the public that is going to be most affected by new and often unfamiliar energy technologies and the subtle changes in lifestyle they are almost certain to bring about. 


\section{New Facilities and Equipment}

As the United States begins to adapt these technologies on a widening scale, enormous investments in facilities, equipment and trained manpower are going to be needed in the years ahead. Table 4 gives the number of major facilities that, according to one of our scenarios, may be required by the year 2000 .

The listing is not additive because different technologies serve the equivalent energy markets in different ERDA scenarios.

TABLE 4. Major energy facilities potentially required by the year 2000

Facilities

Coal-fired power plants

(1000 MWe equivalent)

Light-water reactor power plants

(1000 MWe equivalent)

Breeder reactor power plants

(1000 MWe equivalent)

Geothermal power plants

(100 MWe equivalent)

Solar electric power plants

(100 MWe equivalent)

Coal mines

(millions of tons/yr equivalent)

Oil from shale plants

(50,000 b/d oil equivalent)

Coal liquefaction or gasification

plants

(50,000 b/d oil equivalent)

Solar heated and cooled buildings (millions)

Heat pumps (millions)

Electric automobiles (millions)
Potential number required

220

370

80

400

$200-400$

2,000

80

140

10-15

26

15 


\section{Cooperative Federal Role in Energy Development}

Because the development of new energy technologies and the facilities to exploit them requires long lead-times with attendant economic and other uncertainties, potential customers may be reluctant to provide investment capital when it is most needed. Close Federal cooperation with the private utility and production sectors, therefore, has been and is being encouraged.

Such cooperation can include risk-sharing during the critical phases of research and technology development, price supports coupled with nonrecourse guaranteed loans, accelerated depreciation, investment tax credits, and the enactment of favorable regulatory policies. Government help is also crucial in reconciling the operational characteristics of new, energy systems with environmental, health, safety, and other requirements.

Also, on their own, Federal centers and laboratories conduct RD\&D that can substantially contribute to the overall energy picture.

\section{ERDA-NBS Programs}

Many Federal centers and laboratories are involved, not only those at tached to ERDA. Of the agencies active in the energy field the National Bureau of Standards is conspicuous for the breadth of its contributions. In fiscal year 1976 alone, ERDA transferred to the NBS $\$ 5.8$ million in spending authority which has been added to a $\$ 3$ million carry-over. With this funding, a total of 44 separate projects grouped in five major program areas is now being supported.

Approximately $\$ 1.3$ million have been allocated to evaluating energyrelated inventions submitted by individual inventors and small businesses. After working their way through an intensive screening process, attractive candidates may be given ERDA development support. Of the 3,000 inquiries received up to mid-February 1976, 1,200 have passed the screening review established by the Office of Energy-Related Inventions. Of these, 400 were selected for first-stage evaluation, 200 already have been evaluated, and 25 succeeded in reaching second-stage evaluation. Thirteen of this group subsequently entered the process and five by mid-February worked their way through yielding two inventions that have been recommended to ERDA for support.

In another program area, some $\$ 2.4$ million have been budgeted for such fossil energy projects as materials research related to MHD on the electrical conductivity, viscosity, and chemistry of coal slags, the development of test methods to determine erosion and stress corrosion of metals in coal gasification plants, and the study of the damage of catalysts used for fuel cells. 
Within the conservation area, supported by $\$ 1.8$ million, the NBS is evaluating the performance of a test facility heated by solar collectors and at the same time is studying the energy performance of buildings to determine how much insulation and other energy-related modifications they may require for maximum fuel economy. For industrial use, data are generated and improved methods are developed to measure flow rate, temperature, and chemical composition of hot gases and flames in efforts to yield more efficient combustion. Tests of the substitution of waste products have also been carried out in paper and cement production.

Another area of great importance to ERDA is energy distribution and transmission. This involves about $\$ 1.2$ million dispersed among such NBS projects as the evaluation of instruments and methods to measure electric fields near high-voltage transmission lines and the investigation of insulating materials that may be used for super-conducting underground electric transmission. Another $\$ 2$ million is represented by NBS' nuclear activities, including the calibration of new types of neutron exposure dosimeters used by reactor personnel, and the gathering of atomic and molecular data related to magnetic confinement and laser-induced fusion.

These are but examples of the important work the National Bureau of Standards is undertaking - work that contributes significantly to America's energy programs while strengthening the scientific and technological prestige of the United States.

After a decade or more of questioning the meaning and worth of science and technology, Americans are now realizing that of themselves they are neither good nor evil. How science and technology are employed, the purposes to which they are put, are what count.

On an ever-broadening scale, science and technology are being applied to improve the condition and to assure the integrity of our 200-yearold nation. In late March, President Ford again urged that Congress approve his $\$ 24.7$ billion budget for research and development in the fields of energy, defense and space, an 11 percent increase over his 1976 estimates. In the energy area, he requested a 35 percent increase in ERDA's budget authority for fiscal year 77 over that of FY 76 .

Half of the increase, $\$ 805$ million in budget authority and $\$ 613$ million in outlays, is for energy research, development and demonstration. Reflecting the importance he attaches to science and technology, the President has requested that Senate and House bills relating to the establishment of the White House Office of Science, Engineering and Technology Policy be acted upon as soon as possible.

Contrary to the idle dreams of a few, our nation and all other nations are going to demand vast quantities of energy for as far into the future as one is able to predict. What we are setting out to do today will make that future secure, for without energy, society as we conceive it would crumble. President Ford said recently, "I fully recognize that this country's fu- 
ture - and that of all civilization as well, depends on nurturing and drawing on the creativity of men and women in our scientific and engineering community." The truth of this is compelling. There can be no turning back to the simpler nonscientific, nontechnological ways of the past - if, indeed, they were simpler.

\section{References}

[1] Schurr, S. H. and Netschert, B. C., Energy in the American Economy, 1850-1975 (Baltimore: Johns Hopkins University Press, 1960).

[2] Miller, B. M. et al., "Geological Estimates of Undiscovered Recoverable Oil and Gas Resources in the United States," U.S. Geological Survey Circular 725 (Reston, Virginia: U.S. Geological Survey, 1975).

[3] Energy Reorganization Act of 1974, Public Law 93-438, 93d Congress, H. R. 11510 (Oct. 11, 1974).

[4] Federal Nonnuclear Energy Research and Development Act of 1974, Public Law 93-577, 93d Congress, S. 1283 (Dec. 31, 1974).

[5] "A National Plan for Energy Research, Development \& Demonstration: Creating Energy Choices for the Future. Volume I: The Plan; Volume II: Program Implementation," Report: ERDA-48 (Washington: Energy Research and Development Administration, 1975).

[6] "A National Plan for Energy Research, Development \& Demonstration: Creating Energy Choices for the Future. Volume I: The Plan; Volume II: Program Implementation," Report: ERDA-76 (Washington: Energy Research and Development Administration, 1976).

[7] "Update: Nuclear Power Program Information and Data," (Washington: Energy Systems Analysis, Division of Reactor Research and Development, Energy Research and Development Administration, Jan. 1976).

[8] "Uranium Industry Seminar, October 1975," ERDA Report GJO 108 (1975). Available through Bendix Field Engineering Corp., P.O. Box 1569, Grand Junction, Colorado, 81001. Also Patterson, J. A., "Uranium Supply Requirements," presentation given at Atomic Industrial Forum Fuel Cycle Conference, Phoenix, Arizona (Mar. 22, 1976). (ERDA Division of Nuclear Fuel Cycle and Production).

[9] Averitt, P., "Coal Resources of the United States, January 1, 1974," U.S. Geological Survey Bulletin 1412 (Washington: U.S. Geological Survey, 1975).

[10] "Geothermal Energy Research, Development \& Demonstration Program: Definition Report," Report: ERDA-86 (Washington: Division of Geothermal Energy, Energy Research and Development Administration, Oct. 1975).

[11] White, D. E. and Williams, D. L., eds., "Assessment of Geothermal Resources of the United States-1975," U.S. Geological Survey Circular 726 (Arlington, Virginia: U.S. Geological Survey, 1975).

[12] "National Solar Energy Research, Development \& Demonstration Program: Definition Report," Report: ERDA-49 (Washington: Division of Solar Energy, Energy Research and Development Administration, June 1975).

[13] "National Program for Solar Heating and Cooling," Report: ERDA-23A (Washington: Division of Solar Energy, Energy Research and Development Administration, Oct. 1975).

[14] "R\&D Programs Summary," (Washington: Division of Oil, Gas \& Shale Technology, Energy Research and Development Administration, Mar. 1976). 
[15] "Recommendations for a Synthetic Fuels Commercialization Program" (vols. I, II, III, IV), Report submitted by the Synfuels Interagency Task Force to the President's Energy Resources Council (Washington: U.S. Government Printing Office, vols. I, II, and III, Nov. 1975; vol. IV, Dec. 1975).

[16] "Liquid MetaI Fast Breeder Reactor Program-Overall Plan," Report: ERDA-67 (Washington: Division of Reactor Research and Development, Energy Research and Development Administration, Dec. 15, 1975).

[17] "Liquid Metal Fast Breeder Reactor Program-Final Environmental Statement," vols. I, II, and III, Report: ERDA-1535 (Washington: Energy Research and Development Administration, Dec. 1975).

[18] "Fusion Power Research and Development Program Projections," (Division of Controlled Thermonuclear Research, Energy Research and Development Administration, May 1975). Also Hirsch, R. L., "Status and Future Directions of Fusion Power Research and Development," presentation given at Third Energy Technology Conference, Washington, Mar. 29, 1976. (ERDA Solar, Geothermal and Advance Energy Systems, S-7-76). 



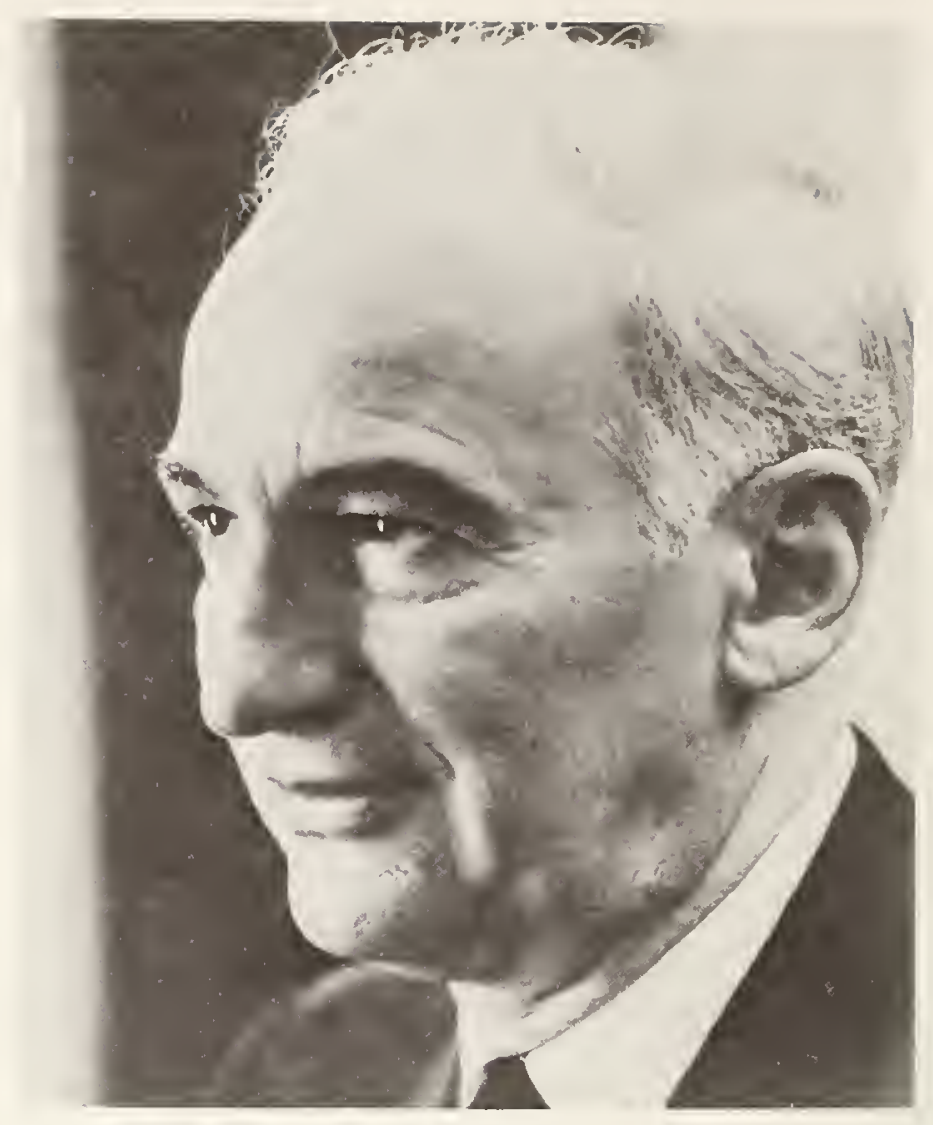

Dr. Simon Ramo is Vice Chairman of the Board and Chairman of the Executive Committee of TRW Inc. He is also one of the key advisors to the government on technological matters, his assignments having included membership on the White House Energy Research Advisory Council and the Secretary of State's Advisory Committee on Science and Foreign Affairs. He is now Chairman of the President's Committee on Science and Technology and a member of the Advisory Council to the Secretary of Commerce. Dr. Ramo was the Chief Scientist for the nation's intercontinental ballistic missile program. He has been a cofounder of several successful technological corporations. Holder of numerous awards and honorary doctorates, he is a founding member of the National Academy of Engineering, a member of the National Academy of Sciences and the author of texts widely used in universities and by practitioners. 


\section{THE FUTURE ROLE OF ENGINEERING}

A common and short definition of engineering describes it as the application of science and technology to the benefit of mankind. On this 75th anniversary of the National Bureau of Standards, an institution which has contributed so much to the nation through its engineering expertise, it is pertinent to ask: Is the United States employing science and technology to the fullest on behalf of our society? This is not the same as inquiring: Are we following up every clue to nature's undiscovered secrets and are we building every machine it is technically possible to build? These latter are very different (and partly foolish) questions. We seek here to know rather whether our scientific and technological know-how is being applied avidly where strong potential exists for a net, high social reward for the effort.

To this serious question I submit the answer is "no." While our nation today has more capability in science and technology than ever before, we are using it less-less as a fraction of the full, beneficial possibilities. Science and technology, fully employed, could improve the value of our resources, natural and human. These tools could be put to work to develop additional products so economically and socially advantageous as to warrant the investment of the required resources and whose production could create new jobs to reduce unemployment. Further research could lead us to methods for increasing supply and lowering costs as a counter to inflation, substitutes for materials in short supply, and ways of acquiring raw materials and manufacturing what we need with less harm to the environment. But we have become slower, more timid and less innovative in applying science and technology toward such possible ends.

Lethargy and negativism regarding research and development are especially penalizing to the U.S. society because our values and habits are so strongly based on a generous availability of the fruits of advancing science and technology. Our high standard of living is rooted in many decades of such advance. While some folks may prefer a life less dependent on high production of goods and service, political experts assure us that no approach to our social and economic problems is politically viable if it contemplates the average citizen's accepting a substantially reduced personal supply. It is equally unrealistic politically to expect those living in substandard conditions to give up aspirations for a better life. If these are political truths, then as practical corollary truth, the tools of science and technology must be used vigorously, because such use is indispensible for a feasible approach to national problems. 
Of course, when we speak here of employing technology, we specifically do not mean the unthinking application of it, the mis-use of technology on projects and products the public does not in the end really want and that bring us more harm then benefit. A high rate of technological advance we recognize as not automatically synonymous with happiness. Also, we know that even if all implemented technological programs were selected to please the great majority of citizens, we still would not be guaranteed a healthy economy and happy society. If the government and the public handle badly other (nontechnology related) decisions, we can have inflation, recession, unemployment, high pollution, urban problems, and numerous other ills all at once. However, without a strong engineering foundation, our needs will not be satisfied. Science and technology are not sufficient. But they are necessary.

They are essential to the United States for more than domestic tranquility. The U.S.'s international economic competitiveness and our contribution to world stability and progress are both dependent on the status of our science and technology. If world social and economic health is best fostered by each nation doing for the world what it can do best and freely trading with the other nations for their most suppliable items in return, then continuing U.S. advances in science and technology are fundamental to our doing our part. In this regard the situation is again disappointing. In those high technology areas where we are close to unique in our capabilities and opportunities we are moving painfully slowly.

Now, why are we not doing the best we can to reap the potential rewards of scientific research and technological effort? One reason is the growing "anti-technology" wave. A substantial fraction of our nation's citizens equate technology with the devil. In attaining our high production of goods and services, as they perceive it, we have lost much and gained too little: We have been forced to crowd into the cities, this before we have learned to live together. TV offers vapid programs loaded with violence that mis-educate our children. The automobile kills 50,000 people a year and fouls the air and we drive in worsening traffic congestion. We go to the moon but we do not care for our senior citizens. The atom bomb may destroy civilization. The nuclear reactor may poison our environment. We make our soil more productive, but insecticides may do us in.

Some will argue that the people who believe these things are failing to distinguish between the tools of man and his use-his mis-use, rather - of those tools, and we need only to explain the difference to them. Unfortunately, however, there indeed has been misapplication of technology. Our national mechanism for choice and decision-for matching potential to needs and balancing gains and losses when assigning resources to technological advance - is not yet adequately developed. After all, we find it hard to articulate what kind of a society we want. Thus, it is not surprising that it is difficult to pinpoint the most effective application of science 
and technology as tools to help us build it. However, a broad anti-technology bias is a handicap. It stands in the way of our arriving at meaningful value judgments and impairs our overall ability to reach objective, sound, nonemotional decisions on the use of technology.

An even more serious limitation to our wise employment of science and technology is the public confusion as to the right roles for free, private enterprise, on the one hand, and government sponsorship and control, on the other. Thus, many people are convinced "business" is socially irresponsible. Whenever any kind of a problem surfaces, they are certain private enterprise can be counted on only to exploit it, seeking monopolies and "unconscionable" profits at the expense of the consumer. They thus look to government alone to provide solutions. They vote for those politicians who blame business most vehemently. Technological advance is often identified with big business, and the anti-business citizens reason that such "advance" really means injected but unneeded changes, or withheld though needed product improvements. with resulting unemploy. ment and higher prices.

Another large fraction of the voters are equally fed up with government spending and big government generally. They see the government as a huge and increasingly incompetent and inefficient bureaucracy. As to government-sponsored scientific research and technological advance, they see the government as engaging in wasting dollars into the billions, probably in response to numerous selfish-interest constituencies. As a capping indication of the confusion, it seems much of the population holds both of these extreme views at once. They distrust both the free enterprise sector's and government's involvement with technology and can be counted on for a totally negative stance.

We see in the United States today a severe mismatch between the high potential of technological advance and the slow pace of the country's social-political progress. We are simply not organized to use science and technology to the fullest. The problem of wise, full use of science and technology lies not in any lack of availability or promise of science and technology per se. It is the interface of technological with nontechnological factors that is critical and controlling. The whole is a "systems" problem. For instance, in choosing where and how to apply science and technology it would be helpful to have clearer national goals. For a "systems approach" it is required that we know what we are after before we expect to get very far. We cannot make satisfactory decisions on what to do without an understanding of trade-offs and options. We need to be in a position to compare the "good" that can come from technological advance against the "bad" and the cost. As to our employment of technological change, we can be likened to a bunch of carpenters, sawing and hammering away, often getting our fingers in the saws and hitting our own thumbs and each other's heads as we swing our hammers, who don't know quite what we are trying 
to build, who sense an unsatisfactory situation, and who meanwhile blame the saws and hammers.

We have two mechanisms for getting things done in the United States: free enterprise and government. These approaches are not mutually exclusive. In fact, we have always had a hybrid system and the need for a balance as we go about allocating assignments to private or government initiatives. One of the two routes for progress, the free enterprise system - private capital at risk seeking a return - has served well for two centuries to connect many of the needs and desires of the citizens with the capabilities of science and technology. This wedding has blessed us with an immense stream of products and services. Why is this free market, free enterprise mechanism not still the practical answer to the challenge of putting science and technology to work sensibly and completely?

One important reason is that technological businesses producing goods and services, large and small, are not earning enough now on the average to provide adequate funds for investment in scientific research and new technology. After the typically small difference between selling price and costs is used to pay taxes, the interest on borrowed money, dividends to the shareholder, and the inflated costs of replacing depreciating facilities, too little is left to improve methods and develop new products. So dislocating has been the effect of inflation and recession that most corporations have overborrowed. They now find themselves with too high a debt in relation to their equity capital and their net earnings. Since this condition has been paralleled by high interest rates, their debt expenses are extraordinary. Many technological corporations' stock is selling on the market at less than the book value of assets, even though those assets, because of inflation, could not be bought or replaced now at the stated book value. This means new equity capital is as hard to come by as are earnings for reinvest ment.

There are a number of further limitations of the free enterprise sector in helping us realize today the full promise of advanced technology. An increasing proportion of potentially beneficial technological projects now involve too great a business risk. More specifically, the "risk-to-return" ratio is too great. The clearly foreseeable start-up costs are too high, the time to "turn-around" to an eventual profit phase too long, and the dependence of success on political decisions forbiddingly severe. The market is far from being a free one determined by the public, the ultimate consumers. Instead, it is a chaotic cacophony of semi-autonomous, conflicting private and government actions.

To ensure the proper application of science and technology in the interests of the public, the government must be in the act. It must regulate against impairment of the public welfare that might arise from some technological applications, particularly if performed for the narrow benefit of a private group. Moreover, to advance some aspects of science and 
technology for the general society's benefit, the government must be relied upon for a strong and essential if not a solitary contribution. This is perhaps most obvious in the development of military weapons systems, for sending a man to the moon, and for similar large projects considered as vital to the national interest but where there is no consumer product, no free market involving the greneral public. On the whole, scientific and technological advance realistically involves the government either as the doer, sponsor, regulator or partner. Additionally, a host of indirect impacts from necessary and unnecessary government actions set the environment and shape decisions on areas of investment and on the mode and pace of activities in the private sector.

The necessity, and yet the complexity, of combined government and private involvement in science and technology advances means that the pace of the advance depends on organization and cooperation. We are weak in both functions.

In listing factors highly influential in determining whether science and technology are used to the fullest for the public benefit, we must now mention one second to none, namely, the profession that is concerned with this issue. "Engineering" has meant to most, both in theory and in implementation, the utilization of resources to design and build machines and systems. We are in transition to a new, more highly technological society for which this definition of engineering, and the profession and activities it describes, falls far short of meeting that society's requirements. The proper use of science-its timely and wise application to help man with his problems, enchance his opportunities, provide him with acceptable options, and satisfy his social and economic requirements - now is seen to constitute an endeavor of vast proportions. It is this broad effort, the overall matching of scientific and technological advance to social needs and progress that must constitute "engineering." The profession should include, but does not today, everything from the recognition of need, articulating of options for filling it, and analysis of technical-economic-social tradeoffs, to the planning, arranging and actual implementation of the most sensible response - this whenever science and technology play a major part in the accomplishment. If this new profession is not to be developed from present engineering as a beginning base, if engineering cannot rise to this needed "greater engineering" plateau, this failure does not decrease the requirement. We have a missing profession.

We wish now to discuss the many changes in education, image, motivation, practice and organization required to develop this necessary but presently nonexistent profession. This can best be done if we first take a look at some examples of technological advances which the society could attain with benefit if we were to go about it correctly, but where the pace, quality, and clarity of effort today is unsatisfactory. 
In the coming decades the world problem of food and nutrition will probably move to the top as a critical issue. With very few exceptions the nations of the world, developed or underdeveloped, face the certainty of food insufficiency problems. The United States is close to unique in possessing the combination of natural and technological resources such as to yield us a permanent large food surplus. The production we are capable of could enable us to make a dominant contribution to the world's supply problem. If we organize our research and technology in relationship to the inarket requirements properly, and, of course, have the right political-social-economic policies, we can improve the economic health and stability of the world, exert world leadership tending towards peaceful, cooperative world trade (and enjoy a favorable trade balance rather similar to the OPEC nations' petroleum based income).

Our land, soil, weather, topology and size of terrain are outstanding, our capacity for high mechanization is great, and our technological knowhow on growing, processing, storing, and distributing food is ahead of the rest of the world. Yet we have hardly scratched the surface. Very basic research questions are unanswered (what are the real nutritional requirements of a human being?). Detailed technology of the entire process from seed to mouth remains skimpy. For a maximum effort both the government's and the private sector's role are not yet fully developed and defined. Water, energy and environmental factors involve the government. Policies for sale of products to other countries certainly do. The private sector can be motivated by the right national policies to a much greater level of investment, innovation and accomplishment.

Many billions of dollars per year of added income to the United States, millions of people saved from starvation and undernourishment, and greater world stability depend on our having the skill and desire to organize agricultural science and technology advance, on our putting to work the know-how and entrepreneurial ability of Americans and our physical resources. It is not a project to be finished in a year or two by a small group of government policy makers. The task involves instead a spectrum of interacting programs including many disciplines and many government and private interests. Food is an area full of challenge for the full utilization of science and technology. But if we are to move rapidly and optimize benefits in relationship to costs, the field needs and deserves a thoroughly professional effort.

Another example where science and technology advances are not being matched well to the national need is in the area of transportation, whether mass public transport in cities, railroads, airlines or automobiles. In each of these and other segments something is wrong. We are not getting the transportation that, speaking only technologically, we should be capable of. Too large a fraction of the operating entities are just barely avoiding bankruptcy or have achieved it, or it is not possible to obtain 
financing for needed improvements, or too much of the traveler's time is dissipated, or the system uses up too much energy, or it pollutes the air too severely - all this beyond what would be the situation if we could organize to create a superior match of technology to real requirement.

Specifically, surely almost every large city could gain by installing a first-class public transportation system. Of course, for this to be so it has to be the right system. The approach has to be carefully selected and suited to each city's layout, industry pattern, employment, health care, education - that is, to that city's total economy, social conditions and life-style. The social and economic gains, assuming a sound application of the correct technology to the city's needs, could be prodigious. In Los Angeles, where I happen to live, the average person resides, say, 10 miles from work - such is the city's "design" - and completes this 10 miles through heavy traffic at something closer to 10 miles an hour than the 100 miles per hour his automobile can do. A combination of private and urban transportation harmonized with the needs of the people would use less energy, cut air pollution, decrease accident rates and require less total investment (most of the latter now left all day in the parking lot contributing nothing). If workers could save hours each week compared with present means for getting to and from work, that would be the equivalent of a major increase in productivity.

Let us assume I am right about there being a need for a public mass transit system in Los Angeles, a potential for large gain in economic and social terms. Would it be intelligent for even the largest of American technological corporations to invest private resources with the objective of developing, then selling, and thus finally earning a return from, a mass transit system for Los Angeles? Hundreds of separate (and generally quarrelling, apathetic, self-seeking and uncooperative) groups, both private and governmental, are involved in the creation of the "market." Critically important and potentially beneficial as the application of the right transportation technology might be to Los Angeles that market is not yet formed. The "risk-to-return" ratio for the private corporation going after this field is absurdly high and the start-up cost is huge. Even assuming success in the vague future, the time to pay-off is too long and the government's policy on transportation fares - and hence, the promise of return on the private investment - is too unpredictable.

Consider next an environmental example. Done with a mixture of creativity and common sense, a major program for selective depolluting of the principal waterways of the nation stands to yield a high return on the investment through improved health, quality of life and long term economic gain from preservation of human and natural resources. To accomplish this on more than a small scale would require much more scientific research and technology effort than we have so far expended. Understanding pollution phenomena in detail, developing superior nonpolluting approaches to 
the use of the waters, and inventing and producing a myriad of specialized equipment would require the assignment of expensive technological and scientific resources for many years. Of course, such specific technical work would be meaningless without attention to complex interface problems, such as severity of cleanliness standards versus short-term unemployment consequences, or puzzling out the long-term value to the society of cleaner water (how clean?). The decision-makers, in the end the public, must be able to see the options and compare the benefits against the price to be paid.

The technical problems in this area of engineering and the trade-off questions of a social and economic nature are admittedly difficult, but the organization problem is even more so. Take a specific example-cleaning up Lake Erie. If a combine could be created of, say, five large corporations, including amongst their staffs and their sub-contractors all the technical expertise required, how could they even presume to design and then go about installing a system that might depollute the Lake but would also affect the economy and hence the social makeup of industrial communities of millions of citizens bordering it? The private combine might offer proposals to install superior waste disposal equipment to a typical city contributing sewage to the Lake but why should that city pay to pollute the Lake less unless all other polluters do their part in an agreed-upon, balanced program. Government initiative and sponsorship, at least in part, and rather complete government regulation of standards are essential to create a market. Of course, a syndicate of large private corporations would not be allowed to form under present interpretations of anti-trust laws, even though such an arrangement might be necessary to achieve the required breadth of attack on the whole system problem.

We are today approaching the various problems of environmental control, whether it be land, water or air, through a highly fragmented system of sponsorship, planning and control. We have arranged nothing near to the degree of cooperation required between the private sector, where much of the science and technology know-how is to be found, and the government agencies, local through national, who are necessary participants.

A quite different example of the relationship between science and technology advance and the filling of national needs is to be found in electronic information technology. Electronic data systems are now possible that can absorb, store, categorize, process, ponder, move and present information in vastly higher quantities, yet with greater speed, radically reduced cost and increased reliability and accuracy, than has ever before been conceivable. This electronic "synthetic brain power" can make each human being smarter at his job. Information makes man's spinning world of activities go around, and we all spend much of our time doing something with information. By a new man-technology partnership in information handling the potential for increasing the value of every hour in a person's time 
and hence the nation's productivity is tremendous - in business and indus try, banks, the professions, airlines, hospitals, educational institutions, and all levels of the government.

We know that, compared with almost every developed nation, the productivity growth in the United States is lagging. If it continues, this lag will increasingly hurt our international competitiveness and stand in the way of growth of standard of living for the many millions in the United States whose situation is well below average. Electronic information technology looms as the solution to the increasing costs in the service sectors of our economy. The counter to inflation and potential for new jobs which this new technology offers makes it especially regrettable if we cannot use science and technology to the fullest in this area.

As with the telephone then the automobile and later TV, such electronic information handling advances become economical only if the users are numbered in the many millions. The United States is close to unique in the world in possessing the combination of characteristics needed for implementation of this information-technology advance: technological lead; a single integrated large market; a clear need; opportunity for high return on invested resources. Almost everyone's output could be improved by the equivalent of say, $\$ 2,000$ a year by the application of innovative electronic technology that would cost about $\$ 1,000$ a year for hardware and software per person using it, that is, if the implementation were done on a mass, national scale. But this would sum up to some hundreds of billions of dollars of implementation costs, too big for any one corporation.

This effort is progressing now in a steady fashion but not nearly at a rate possible if one looks only at technological bottlenecks or of limitations of basic return on financial investment. The problem is that of the leadership for organization of the systems integration of a full utilization of information technology. Admittedly, many corporations are involved already in producing the ideas, systems concepts, apparatus, and the information in electronic form required for the applications to operate. Thus, tellers in many banks now are using electronic information aids to enable them to service each customer more accurately, rapidly and efficiently. A credit purchase at a department store, a reservation at an airline counter, a purchase of securities through a broker - these and numerous other applications of advanced information technology are becoming familiar to us as we observe the human partners in the activity relying on a network of intelligent terminals, computers, electronic information files and communications systems. Full implementation of electronic information systems would radically change the way in which we communicate with each other, altering our present dependence on the transmittal of pieces of paper by the many billions in motion each day across the nation, would substitute electronic fund transfer for most checks and most cash transactions, would alter the role of the Post Office, and would lead to better concepts for 
purchasing, scheduling, manufacture, arriving at decisions of a professional-financial kind, and even the ways medical care is dispensed and schools educate our children. However, for the broadest usc of electronic information technology, the proper interaction of the whole-including the evaluation of standards for performance, privacy, and interconnection, and, again, the arrangement for cooperation between government and private groups - adds up to a system problem far more complex than, let us say, the telephone system or the TV network system of the nation.

In electronic information handling as in many other frontier areas the application of advanced technology is not set by the technology itself but rather by the complexity of the arrangement-making problem.

Space satellites represent startling additional examples of the potential of further beneficial technological advance. For instance, if we want to send telephone messages to Europe we now no longer have to put hundreds of thousands of pounds of copper beneath the Atlantic Ocean in form of a cable. We can instead put a few pounds of copper in a communications satellite and enjoy increased channel capacity. Or consider a space system to advance agricultural technology in which we plot and examine the earth's resources by satellites working with a network of communication equipment, computers and data analyzers on earth. Such a project could enchance weather prediction and hasten mineral and water prospecting as well as disclosing yield potentials and warn of problems for improved agricultural planning. However, a private entity could not scan the terrain of our nation and that of others and then offer the information for sale to realize income without a government sanctioned position to carry out such a task. This means not only government decision-making and sponsorship but also government regulation. Earth resources scanning by satellites is an example of an embryonic, unsettled area where arrangement-making, the settling of goals and functions and the organizing of a team of private and government participants, sets the limit on speed of useful application.

Some aspects of coal technology constitute excellent examples of the problem of advancing technology occurring when the size and risk of the project and the number of independent players become too great. We know we can obtain gas and liquid fuel from coal, that coal can be mined more safely by using new concepts in mining machinery, and that it can be desurfurized and burned more cleanly. However, the complete system needed for a much greater utilization of coal by the United States involves a host of private and public organizations that are rather autonomous and not readily directed from any one point: land owners, mine operators, labor unions, railroads, pipeline companies, power generating and water supply utilities, numerous specialized engineering and manufacturing organizations and many agencies in the federal government and state governments that deal with prices, environmental controls, labor and transport, to name only a few. So far no free enterprise group has risen to make a major entry 
into new coal technology, that is, development then production and distribution on a scale such as to bring coal up to petroleum as a source of energy. Such an entry would require billions of dollars and many years of start up time before a return would be realized on the investment. Indeed that return might never come, so great are the unpredictable risks associated with independent decisions made on related critical activities not subject to the investor's control.

The foregoing examples help illustrate that applying science and technology for the benefit of society is an enormously complex task transcending the science and technology ingredients thereof. Combining knowledge and ideas on so many technical, economic, social and political fronts is an intellectual challenge. The art of mixing together in a harmonious ensemble public value judgments, technical analyses, and creativity with the right content of pragmatic actions surmounts the established expertise of any recognized profession. Yet the potentials match the difficulties. Also, the detriments to society of inadequacy of attack and execution are so great that professionalism in applying science and technology is a vital world need. Amateurism - everybody in the act in a helter skelter free for all-is in some respects inevitable as part of the operations of a democracy. But we are far from an optimum balance. It does not have to be as bad as it is.

Engineering, as the profession is today, does not fill the need. The simple definition that engineering deals with applying science and technology for the benefit of a society is misleading and, presumptuously, though innocently, overstated. Today's engineering deals with only a part of the task, so it is only a part of the missing but required profession. Surely those practicing engineering today cannot on the average be claimed to have a professional knowledge of the society greater than that of many other professionals like lawyers, physicians, educators, businessmen, politicians or those selling insurance. Of course, it should be stated that in no way can any of these other professions be regarded as engaged primarily in the matching of science and technology to society's wants and needs.

Engineering may be closer than other conventional professions to what we must have, but neither in substance nor image does it cover the intellectual disciplines, know-how and interests embracing the whole subject of the wedding of science and technology with economic and social requirements.

True, most engineering educational institutions have for many years required that a typical engineering student include courses in the humanities and social sciences. But this practice has been largely to supply a veneer, a cultural coating to make the engineering graduate a fuller man. It has not been out of a recognition that understanding the way our society operates is as important as understanding physics for the profession the student expects to enter. A number of universities offer "hybrid" courses 
of study-physics and economics, engineering and political science, biology and electronics - and, of couse, some engineering graduates go on to take an additional degree in business, economics or even medicine. Such multiple education equips these graduates for careers that are different from what would be open to them with an engineering degree alone. But the image is not that of creating a force of young people who will comprise the new, presently absent, profession looked to by the public as the source of leadership to cover the science to society relationship. That relationship is being covered instead by accidental, strained, contesting forces powered by people from all walks of life.

Notice how different in some pertinent respects the situation is as regards law and medicine. If we trained our physicians the way we do our engineers we first would define medicine narrowly as involving (if the reader will forgive a slight exaggeration) the application of drugs and knives to the human body. Then we would proceed to train him in drugs and knives also giving him a few short courses on the human body-as a cultural bonus, but not with the imperative claim that knowledge of the body is vital to his professional work. One would think that if engineering is the profession of applying science and technology to society then engineers in sum would have to spend about as much time learning about, and in their professional work dealing with, the society as with the tools they plan to apply to that society.

Public health, whether it involves treatment of individuals, regulation of manufacture and sale of drugs, or organization of government efforts, involves physicians in leadership positions. Physicians are not the total actors in the professional effort of handling the nation's medical and health problems, but they furnish the backbone for it.

In a similar way we certainly look to lawyers on anything that has to do with the law. Attorneys provide the professional foundation for such activities whether they be by government or individuals, businesses or families, and whether in lawmaking, law enforcement or the interpretation of the Constitution. We are not surprised that a majority of national and state senators and representatives and Executive Department office holders have a legal background. The substance and image of the legal profession are both pervasive on all matters of life that relate to the law, even though individual practitioners may narrowly spend their lives on divorces, business contracts, criminal cases or numerous other specialities.

What if there did exist for application of science and technology to society a fully developed, totally adequate professional group with the necessary quality and quantity of practitioners? How would things be different? What would we notice about the way science and technology are applied to the society?

In reply, many things can be mentioned. One broad-brush example is that since ours is a highly technological society, one in which science and 
technology figure into almost every aspect of our lives-the economic strength and security of the nation, the way we move about, communicate, educate ourselves, and provide for our material comfort and maintain our health and happiness - a substantial fraction of the leadership of the nation, private and government, would be expected to come from members of that new profession. Their influence would be felt as the nation goes about reaching decisions. Alternatives would be made clearer to us. Facts, theories, proposals for action, and value judgments would be articulated more clearly. The public would be in the habit of listening and trying to understand what we might get in the way of benefits and have to pay in the way of costs or detriments if we decided on various courses of action. We would expect our railroads, airlines, medical care plans, military weapons systems programs, investments for technological advance and further scientific research projects to be better run or better chosen.

A good many potential changes in the society resulting from technological advance would be anticipated. There would exist no perfect prediction process, naturally, and the public would not have to lose any freedom of choice as a consequence of overplanning. However, we would work less from crises and more by following through based upon an easier and wider participation by the public in choosing directions and emphases as increasing scientific advance and technological developments alter our society. If the people want more time to walk barefoot in the sand rather than to own more pairs of shoes, that would show itself more readily in the motivations and controls the government would set up for environmental conservation, on the one hand, or incentives for productivity improvement, on the other. The average person would have increased awareness that science and technology can be used to realize more the life he wants or, if he does not participate in deciding, might be improperly employed to alter society in a way he does not want. The relationship between goals and operating and decision mechanisms would be clearer, this as a result of a steady flow of analyses and data. A national pattern and habit would develop of expecting a new plateau of quality and quantity through the increased utilization of professional effort now highly inadequately supplied.

Now, if this new level of professional activity is not available today, and if we want it in the future, how do we get there from here? If neither engineering nor any other profession is "it," how do we plant the new seed and make it grow. I submit that engineering is not ineligible even as it exists today as the starting point, although I am not sure that the new profession needs to be called "engineering." Perhaps it is "techno-sociology" or "socio-technology." But those two names are not good enough because they suggest a teaming of only two existing areas of endeavor, engineering and sociology. Perhaps it is better to call it poly-socio-econo-politicotechno-logy or, for short, "polylogy." What we call it is not the most important factor. 
Engineering is a sensible beginning point because of two considerations. First, the already existing base of science and technology in the profession and in the education for it is a basic piece of the substance and image of the required broadened activity. Even more important, engineering is a "do it" profession and the new profession is as well. To digress only slightly, to pursue research, we have need for the kind of individual exemplified at the highest by Einstein. These are people who professionally apply themselves to understand better the laws of nature. This applies not only to physical science but to all other aspects of the universe including trying to comprehend the behavior of man as an individual and in groups. For the new broadened profession we are describing, in the long run it is in dispensible to have a steady flow of the contributions of such fundamental philosophers and researchers. We should not expect to create the missing profession by merging an engineer with, let us say, an academic researcher in sociology. The latter is permanently essential in just what he is doing, at which he is presumably expert and for which he presumably has natural talent and aptitude. The expanded profession we seek to define and create will be concerned, as is today's engineering, with getting things done. Again, like engineering, it is distinct from pure research. It is not concerned with attempting to enhance our understanding of the laws of nature but it will profit and grow as that understanding grows.

University training for this "greater engineering" profession would inevitably have to include three main dimensions: (1) science and technology; (2) the society - the nature of man and his institutions and practical, social-political-economic disciplines related to making the society work; (3) interdisciplinary techniques, or "systems engineering in the large," for attacking problems through recognizing and handling interactions, multidimensional aspects, interfaces, compromises, alternatives, balances and optimizations. The fullest use of science and technology for the nation requires understanding both science and the nation and this thought would dominate the curriculum. In addition, since most real-life problems involve synthesis as well as analysis, methods would be found to bring out the creative as well as the analytical talents of the student.

To assemble a faculty that might be expected to turn out "polylogists" clearly requires a merging of talents and specialities to create a new kind of teacher as well. Obviously "greater engineering," like law or medicine or any major pursuit, will continue to have its highly detailed specialized aspects. Many professionals, probably most of them in fact and most of the graduates, might be expected to pursue specialities as a life's work. The same will apply to faculty members. But there must be an overall concept that all of these specialities are somehow part of an integrated whole which is interdisciplinary. This certainly requires that in the university part of the creation of the new profession there be a strong contribution from individuals whose zeal and capability are the generalization of the whole and the integration of all the parts. 
Certainly for the new profession, if it is to develop as quickly as possible, we shall have to create mergers from various existing professions. There are now people in business, government, law, medicine, sociology, (and even engineering) who agree on the points being expressed here and who, in making their individual contributions, could properly be labeled "school of hard knocks" polylogists. When an engineer becomes a businessman or even conceivably a senator, or a physician becomes a university president, this does not necessarily mean he has become a polylogist, a member of the new profession we have been describing. He may have simply changed from one specialized, existing profession to another neither of which is the new and presently missing profession. In mentioning existing polylogists, I refer rather to individuals whose interests and contribution are in the broadest sense actually in the application of science and technology to the meeting of society's needs. The individuals who are in such situations today are engaged in their interactive, interdisciplinary activities more or less fortuitously. They have not been deliberately trying to create a pattern for a new profession.

If we want to do precisely that, the professional societies could be helpful. Leaders in engineering should be interested in broadening engineering to meet a societal need. Those concerned, even though they are not engineers, with the operation of major science and technology centers also should be interested. So should leaders of the academies and the major philosophical societies, as should university presidents, and heads of technological corporations and of those large government organizations that have a heavy dependence on science and technology.

All engineers might encourage the bringing of people into our fraternity who are not normally thought of as engineers, this if they are engaged in a distinguished way in contributing to the fullest use of science and technology for the benefit of the world. Many consulting engineers come closer in certain respects to having a beginning foundation for "greater en gineering" than do, say, computer engineers or aerodynamists. However, in general all engineers tend to have been organized and categorized by technical specialities rather than through the route of interdisciplinary activities and seldom through the merging and matching of nontechnological with technological aspects.

As these paragraphs are recited as to what might be done to bring the new profession into being, and even as the case is made that present day engineering in substance and image offers the best base for expansion and growth into the needed profession, I am conscious of how little $\mathbb{I}$ have really said about how to get there from here. But I think we should be trying earnestly to make the step because the payoff could be so great.

Perhaps it is inevitable that the new profession will arrive only when it is ready to arrive as a result of eventual demand pressures of the real world. More and more it will become apparent to thinking people that we 
are inadequately using, or in many instances actually misusing, these remakable tools of man, science and technology. It will be seen we are missing too much. Evidence will flow steadily showing what we might gain in rewards by improved vision and performance. The surfacing of the potential will attract outstanding brains. Organizational patterns will begin to improve and intellectual disciplines will be developed. The new profession will evolve. I suspect it may still be called "engineering." Whatever it is called, it will be different-challenging, creative and beneficial to the world, well beyond today's engineering. 


